\title{
NEUE HERLEITUNG UND EXPLIZITE RESTABSCHÄTZUNG DER RIEMANN-SIEGEL-FORMEL
}

\author{
Dissertation \\ zur Erlangung des Doktorgrades \\ der Mathematisch-Naturwissenschaftlichen Fakultät \\ der Georg-August-Universität zu Göttingen
}

vorgelegt von

Wolfgang Gabcke

aus Bremerhaven

Göttingen 1979 


\begin{abstract}
Die asymptotische Entwicklung der Funktion $Z(t)=e^{i \vartheta(t)} \zeta(1 / 2+i t)$ für reelle $t \rightarrow+\infty$ (dabei ist $\vartheta(t)=\Im \log \Gamma(1 / 4+i t / 2)-(t \log \pi) / 2$ und $\zeta(1 / 2+i t)$ die Riemannsche Zetafunktion auf der kritischen Geraden $\Re(s)=1 / 2)$ - heute allgemein als Riemann-SiegelFormel bezeichnet - wird auf neue Weise mit Hilfe der Sattelpunktmethode aus der sogenannten Riemann-Siegel-Integralformel hergeleitet. Die Formeln zur Berechnung der in der asymptotischen Reihe auftretenden Koeffizienten werden vereinfacht und für $t \geq 200$ explizite Fehlerabschätzungen für die ersten 11 Partialsummen dieser Reihe angegeben.

Der tabellarische Anhang enthält u. a. die exakte Darstellung der ersten 13 Koeffizienten der asymptotischen Reihe in der auf D. H. Lehmer zurückgehenden Form sowie die Potenzreihenentwicklungen und die Entwicklungen nach Tschebyscheffschen Polynomen 1. Art der ersten 11 Koeffizienten mit einer Genauigkeit von 50 Dezimalstellen.

The asymptotic expansion of the function $Z(t)=e^{i \vartheta(t)} \zeta(1 / 2+i t)$ for real $t \rightarrow+\infty$ where $\vartheta(t)=\Im \log \Gamma(1 / 4+i t / 2)-(t \log \pi) / 2$ - today known as Riemann-Siegel formula - is derived in a new and simpler way. Simplified computation formulas of its coefficients are given as well as explicit error estimates of its first 11 partial sums for $t \geq 200$.

In an appendix the first 13 coefficients of the asymptotic series are presented in a form introduced by D. H. Lehmer in 1956. Power series and expansions in terms of Čebyšev Polynomials are given for the first 11 coefficients to 50 decimals.
\end{abstract}

Keywords: Riemann zeta-function, Riemann-Siegel formula, explicit estimates of the remainders, power series of the coefficients.

\title{
Referent: Professor Dr. M. Deuring
}

Korreferent: Professor Dr. H.-W. Burmann

Tag der mündlichen Prüfung: 15. Februar 1979 


\section{Inhaltsverzeichnis}

Vorwort

\begin{tabular}{lr}
\hline Einleitung & 1
\end{tabular}

\begin{tabular}{|llll}
\hline 1 & Formale Herleitung der Riemann-Siegel-Formel & $\mathbf{7}$
\end{tabular}

1.1 Definition und Integraldarstellung von $Z(t)$. . . . . . . . . . . . . 7

1.2 Anwendung der Sattelpunktmethode $\ldots \ldots \ldots$. . . . . . . . . 9

1.3 Die Riemann-Siegel-Formel $\ldots \ldots$. . . . . . . . . . . . . . . . . 12

$2 \quad$ Die Koeffizienten der Riemann-Siegel-Formel 19

$2.1 \quad$ Formeln zur Koeffizientenberechnung. . . . . . . . . . . . . . . . . . . 19

$2.2 \quad$ Potenz- und T-Reihen der Koeffizienten $C_{n}(z) \ldots \ldots$. . . . . 31

$2.3 \quad$ Abschätzungen von $C_{n}(z)(0 \leq n \leq 10,|z| \leq 1) \ldots \ldots$. . . . . . 35

$3 \quad$ Die Riemann-Siegel-Formel mit Restglied $\quad 41$

3.1 Restabschätzung der asymptotischen Reihe von $S$. . . . . . . . . . . 41

3.2 Explizite Abschätzung der ersten 11 Restglieder . . . . . . . . . . . . 53

3.3 Zur Divergenz der Riemann-Siegel-Formel . . . . . . . . . . . . . . . 58

\begin{tabular}{|lll}
\hline 4 & Hilfssätze und Hilfsabschätzungen & 61
\end{tabular}

4.1 Integralformeln . . . . . . . . . . . . . . . . . . . . 61

$4.2 \quad$ Die asymptotische Entwicklung von $\vartheta(t) \ldots \ldots$. . . . . . . . 69

$4.3 \quad$ Bestimmung von $\mathcal{D}_{0}(\tau)$ und $\left.\mathcal{D}_{1}(\tau)\right] \ldots \ldots$. . . . . . . . . 76

4.4 Verschiedene Hilfsabschätzungen $\ldots \ldots$. . . . . . . . . . . . 80

\begin{tabular}{lr}
\hline Literaturverzeichnis & 87
\end{tabular}

$\begin{array}{lr}\text { Tabellen } & 91\end{array}$

I. Primfaktorzerlegung der Zahlen $a_{k}^{(n)}(0 \leq n \leq 11)$. . . . . . . . . . 91

II. Primfaktorzerlegung der Zahlen $d_{k}^{(n)}(0 \leq n \leq 12) \ldots$. . . . . . . . 95

III. $\quad$ Exakte Darstellung der Koeffizienten $C_{n}(z)(0 \leq n \leq 12)$. . . . . . . 97

IV. Potenzreihen der Koeffizienten $C_{n}(z)(0 \leq n \leq 10) \mid \ldots \ldots 1$

V. Tschebyscheffreihen der Koeffizienten $C_{n}(z)(0 \leq n \leq 10)$. . . . . 113 



\section{Vorwort}

Vor 36 Jahren habe ich meine Dissertation in einer Form veröffentlicht, die den heutigen Ansprüchen an eine mathematische Publikation - Satz in $\mathrm{T}_{\mathrm{EX}}$ und Bereitstellung als PDF-Datei - nicht mehr gerecht wird. Auch die Beschaffung eines Exemplars des Drucks von 1979 ist wohl vor allem für ausländische Interessenten ausgesprochen schwierig und mit großem Aufwand verbunden, wie ich von Juan Arias de Reyna weiß. Hauptsächlich aus diesen beiden Gründen habe ich mich entschlossen, meine Dissertation mit $\mathrm{LTT}_{\mathrm{E}} \mathrm{X}$ neu zu setzen und in Form einer PDFDatei allgemein zur Verfügung zu stellen. Weitere Beweggründe waren, daß die in der Arbeit verwendeten Methoden nebst den daraus gewonnenen expliziten Abschätzungen der Restglieder bis heute aktuell sind, wie die zahlreichen Zitate der Arbeit in den letzten Jahrzehnten gezeigt haben. Diese doch recht umfangreiche Beachtung durch die mathematische Fachwelt habe ich 1979 nicht erwartet.

\section{Methoden und Ergebnisse der Dissertation in anderen Publikationen}

Einige von den Publikationen, die auf meine Methoden zurückgegriffen oder die die Ergebnisse der expliziten Restabschätzung insbesondere für numerische Berechnungen verwendet haben, möchte ich hier kurz vorstellen. Sie geben z. T. über die Riemann-Siegel-Formel hinausgehende Einblicke in den umfangreichen Themenkomplex „Riemannsche Zetafunktion“, ganz besonders aber in die numerische Berechnungsproblematik dieser Funktion.

Arias de Reyna hat 2011 [3] die Methoden meiner Dissertation auf Geraden $\Re(s) \neq 1 / 2, \Im(s) \rightarrow+\infty$ verallgemeinert und gelangt so zu formelmäßigen Abschätzungen für die Restglieder der asymptotischen Reihe. Dabei geht er ebenfalls von der Riemann-Siegel-Integralformel 4.1.6, S. 65 und nicht von dem komplexen Schleifenintegral (9), S. 3 aus. In einer bisher noch nicht erschienenen Folgearbeit soll eine vollständige Fehleranalyse präsentiert werden.

In [5] stellen Berry und Keating 1992 eine alternative, ebenfalls asymptotische Berechnungsformel für $Z(t)$ vor und vergleichen sie mit der Riemann-Siegel-Formel. Bei gleicher Anzahl der berücksichtigten Summanden ist ihre Formel genauer, erfordert aber einen höheren Rechenaufwand pro Summand. Sie wird heute als BerryKeating-Formel bezeichnet. Den vermuteten Zusammenhang zwischen den Nullstellen von $Z(t)$ und den Eigenwerten eines potentiellen, zu einem chaotischen quantenmechanischen System gehörenden hermiteschen Operators untersuchen Berry und Keating in [7] 1999. Auch dort findet man ausführliche Informationen zur Riemann-Siegel- und zur Berry-Keating-Formel.

Borwein, Bradley und Crandall haben im Jahre 2000 in ihrer Publikation [8] 
neben der Riemann-Siegel-Formel eine Reihe anderer Berechnungsverfahren für die Zetafunktion in einem Kompendium zusammengetragen. Zusätzlich findet man hier viele z. T. kuriose Reihen- und Kettenbruchentwicklungen, die auf unterschiedliche Weise mit der Zetafunktion zusammenhängen.

Von verschiedenen Autoren sind in den Jahren von 1981 bis 2004 umfangreiche numerische Überprüfungen der Riemannschen Vermutung durchgeführt worden. Hier sind vor allem Brent, van de Lune, te Riele, Winter 9. 10. 24], Odlyzko [27], Gourdon/Sebah [18] und Gourdon [19] zu nennen. In jeder der aufgeführten Veröffentlichungen wurde zumindest eine der Restabschätzungen aus Satz 3.2.2. S. 54 verwendet oder zitiert.

In Zusammenhang mit der Primzahlfunktion $\pi(x)$ gab es einige bemerkenswerte Ergebnisse. Mit Hilfe der Riemann-Siegel-Formel hat te Riele 1987 [29] die ersten 50000 Nullstellen von $Z(t)$ mit hoher Genauigkeit berechnet. Zusammen mit dem Nachweis von Rosser et al. aus dem Jahre 1968 [30, daß die ersten 3,5 Millionen Nullstellen dieser Funktion reell sind, konnte er damit zeigen, daß es in der Größenordnung von $7 \cdot 10^{370} x$-Werte gibt, an denen die Differenz $\pi(x)-\operatorname{li}(x)$ positiv $\sqrt{1)}$ ist. Eine analytische Methode zur Berechnung von $\pi(x)$ und einiger anderer zahlentheoretischer Funktionen haben Lagarias und Odlyzko [21] 1987 angegeben.

\section{Offen gebliebene Fragen in der Originalarbeit}

Einige offene Fragen, die ich seinerzeit nicht lösen konnte, sind inzwischen geklärt. So hat Arias de Reyna 2003 [2] bewiesen, daß die Zahlen $\lambda_{n}, \varrho_{n}$ und $\mu_{n}$ aus Satz 4.3.1. S. 76 und daher auch die $d_{k}^{(n)}$ aus Satz 2.1.1. S. 19, wie schon von mir vermutet, ganzzahlig sind (für die $\varrho_{n}$ und $\mu_{n}$ hatte ich diese Vermutung nicht explizit formuliert).

Die in Abschnitt 3.3. S. 58 aufgeworfene Frage, ob die Riemann-Siegel-Formel für jedes feste $t>0$ divergiert oder nicht, konnte von Berry 1995 [6] geklärt werden. Demnach liegt immer Divergenz vor - das war aufgrund der Vorgehensweise bei der Herleitung der asymptotischen Reihe auch nicht anders zu erwarten -, so daß diese Reihe tatsächlich „nur" eine „asymptotische Entwicklung“ darstellt. Berry macht auch Angaben zum minimal erreichbaren Abbruchfehler, den eine Berechnung mit der Riemann-Siegel-Formel bei vorgegebenem $t>0$ erzeugt, wenn die Summation der asymptotischen Reihe bis zum betragskleinsten Glied fortgeführt wird. Er zeigt, daß dieser Fehler mit $e^{-\pi t}$ genau in der Größenordnung liegt ${ }^{2}$, die auch der „Eingangsfehler“ der Riemann-Siegel-Formel aufweist, der durch Weglassen des Ausdrucks 0.5 $\arctan e^{-\pi t}$ in Formel 4.2.3 (a), S. 71 unweigerlich entsteht. Demnach ist das Divergenzverhalten der Riemann-Siegel-Formel dem der Stirlingschen Reihe für $\log \Gamma(t / 2)$ sehr ähnlich ${ }^{3)}$ und damit auch die maximal erreichbare Genauigkeit in Abhängigkeit vom Argument.

\footnotetext{
1) Littlewood zeigte schon 1914, daß diese Differenz für $x \rightarrow \infty$ unendlich oft das Vorzeichen wechselt. Die von te Riele angegebene Größenordnung konnte inzwischen auf etwa $10^{316}$ reduziert werden.

2) Eigene Berechnungen mit den ersten 132 Gliedern der asymptotischen Reihe haben das bestätigt.

3) Statt die jeweiligen Abbruchfehler direkt zu betrachten, müßte eine geeignete Mittelung von ihnen über die $t$-Intervalle $\left[2 \pi N^{2}, 2 \pi(N+1)^{2}\right]$ für $N$ ganz $\geq 1$ - hier hat die Hauptsumme genau $N$ Glieder - zum Vergleich herangezogen werden, da der Abbruchfehler beim Durchlaufen eines solchen Intervalls lokal sehr stark schwankt. Er kann dabei sogar das Vorzeichen wechseln und deshalb an bestimmten Stellen des Intervalls verschwinden.
} 
Ungeklärt ist nach wie vor die nicht triviale Frage, ob der $C_{0}$-Term zusammen mit der Restabschätzung $\left|R_{0}(t)\right|<0.127 t^{-3 / 4}$ für $t \geq 200$ zur eindeutigen Bestimmung von sign $Z(t)$ immer ausreicht. Alle bis jetzt mit der Riemann-Siegel-Formel durchgeführten Berechnungen scheinen das zu bestätigen, zumindest ist mir kein Gegenbeispiel bekannt. Ein allgemeingültiger Beweis dafür liegt aber wohl außerhalb der heutigen mathematischen Möglichkeiten.

\section{Abweichungen von der Originalarbeit}

Ein wörtlicher Nachdruck der Originalarbeit von 1979 kam aus unterschiedlichen Gründen nicht in Frage. So galt es, Druckfehler zu korrigieren, sprachliche Bereinigungen vorzunehmen, das Literaturverzeichnis auf den neuesten Stand zu bringen und vor allem die Möglichkeiten des maschinellen Satzes in $\mathrm{LTT}_{\mathrm{E}} \mathrm{X}$ zu nutzen. Zwei Druckfehler, auf die mich Juan Arias de Reyna aufmerksam gemacht hat, wurden beseitigt und mittels Fußnoten gekennzeichnet. Nicht dokumentiert sind sprachliche Glättungen und die andere Art der Referenzierung von Sätzen und Formeln, die auf die Verwendung des $\mathrm{ATT}_{\mathrm{E} X} \mathrm{X}$-Satzsystems zurückzuführen sind. Das Literaturverzeichnis wurde aktualisiert und erweitert sowie einige dem besseren Verständnis dienende Fußnoten eingefügt. Beides wurde nicht eigens kenntlich gemacht.

Die an mehreren Stellen verwendeten Formulierungen "scharfe Abschätzung“ und "scharf abgeschätzt" waren mißverständlich und wurden kommentarlos mit „optimale Abschätzung“ bzw. „entsprechend genau abgeschätzt“ ersetzt. Mit einer „optimalen Abschätzung" ist eine Abschätzung gemeint, die keine wesentliche Verbesserung mehr erlaubt.

Die unnötige Einschränkung $q>-\sqrt{\pi}$ in Kapitel 3, S. 41 und in Satz 4.4.4. S. 85 wurde beseitigt, so daß die dortigen Abschätzungen nun auch für $q=-\sqrt{\pi}$ gelten.

In Satz 4.3.1. S. 77 sind jetzt auch die numerischen Werte der ersten sieben Zahlen $\varrho_{n}$ wiedergegeben, die zwar 1979 implizit schon vorhandenen waren, deren Abdruck ich aus heute nicht mehr nachvollziehbaren Gründen damals aber nicht vorgenommen habe.

Alle numerischen Werte im Text sowie das gesamte Tabellenwerk im Anhang habe ich nicht der Originalarbeit entnommen, sondern mit selbstgeschriebenen CProgrammen vollständig neu berechnet. Erwähnenswerte Abweichungen von den Werten des Jahres 1979 haben sich dabei nicht ergeben. Dasselbe gilt auch für die Neuberechnung, die Juan Arias de Reyna mit Mathematica für seine spanische Übersetzung [17] durchgeführt hat. Folglich sind mit drei unterschiedlichen Programmen zweier Autoren dieselben numerischen Werte erzeugt worden, was deren Korrektheit hinreichend belegen sollte.

Von den vorstehenden Ausnahmen abgesehen, wurden an der Originalarbeit keine inhaltlichen Änderungen vorgenommen. Das gilt auch für die Rechtschreibung, die den am 01.01.1903 lt. Bundesratsbeschluß vom 18.12.1902 eingeführten Regeln entspricht.

Die vorliegende Fassung meiner Dissertation wird auf einem Server der Universität Göttingen als PDF-Datei zum Herunterladen bereitgestellt. Sie mußte ebenso wie das Original von 1979 in deutscher Sprache verfaßt werden. Eine englische Version wäre natürlich wünschenswert, jedoch habe ich auch nach Ende meines Arbeitslebens nicht die für eine Übersetzung notwendige Zeit. Wie oben bereits 
erwähnt, gibt es aber eine sehr schöne spanische Übersetzung [17], die Juan Arias de Reyna 2003 erstellt hat. Dafür und für seine hilfreichen Anregungen möchte ich mich an dieser Stelle herzlich bei ihm bedanken.

Göttingen, Mai 2015

Wolfgang Gabcke

E-Mail: wolfgang@gabcke.de 


\section{Einleitung}

Im Jahre 1932 veröffentlichte C. L. Siegel [31, 32] in einer Arbeit über Riemanns Nachlaß zur analytischen Zahlentheorie eine asymptotische Entwicklung der Riemannschen Zetafunktion $\zeta(s)$ für festen Realteil von $s$ und $\Im(s) \rightarrow+\infty$. Dabei erweist sich der Fall $\Re(s)=1 / 2$ zur Untersuchung der Riemannschen Vermutung als der bei weitem bedeutenste, auf den wir uns daher in dieser Arbeit beschränken werden. Durch Einführung der für reelle $t$ reellen Funktion

$$
Z(t):=e^{i \vartheta(t)} \zeta\left(\frac{1}{2}+i t\right)
$$

mit

$$
\vartheta(t):=\Im \log \Gamma\left(\frac{1}{4}+i \frac{t}{2}\right)-\frac{t}{2} \log \pi
$$

gelingt es, die Untersuchung der Nullstellen von $\zeta(s)$ auf der kritischen Geraden $\Re(s)=1 / 2$ auf ein reelles Problem zurückzuführen. Die bereits von Riemann gefundene und von Siegel an der oben genannten Stelle veröffentlichte asymptotische Entwicklung von $Z(t)$ für $t \rightarrow+\infty$ wird heute allgemein als Riemann-Siegel-Formel bezeichnet.

In dieser Arbeit werden wir die Riemann-Siegel-Formel auf einem neuen, wesentlich einfacheren Wege herleiten, die Formeln zur Berechnung ihrer Koeffizienten vereinfachen und die ersten 11 Partialsummen der asymptotischen Reihe mit expliziten Restabschätzungen versehen. Bevor wir die Ergebnisse hier im einzelnen angeben, treffen wir folgende Konventionen, die für die ganze Arbeit Gültigkeit haben:

i. Die Quadratwurzel ist stets positiv zu nehmen.

ii. Die Bernoullischen Zahlen $B_{n}$ und die Eulerschen Zahlen $E_{n}$ sind durch die Potenzreihenentwicklungen

$$
\frac{x}{e^{x}-1}=\sum_{n=0}^{\infty} \frac{B_{n}}{n !} x^{n} \quad|x|<2 \pi \quad \text { und } \quad \frac{1}{\cosh x}=\sum_{n=0}^{\infty} \frac{E_{n}}{n !} x^{n} \quad|x|<\frac{\pi}{2}
$$

gegeben. Es ist also $B_{2 n+1}=0$ für $n \geq 1, E_{2 n+1}=0$ für $n \geq 0$ und

$$
\begin{aligned}
& B_{0}=1, B_{1}=-\frac{1}{2}, B_{2}=\frac{1}{6}, B_{4}=-\frac{1}{30}, B_{6}=\frac{1}{42}, B_{8}=-\frac{1}{30}, B_{10}=\frac{5}{66}, \ldots ; \\
& E_{0}=1, E_{2}=-1, E_{4}=5, E_{6}=-61, E_{8}=1385, E_{10}=-50521, \ldots
\end{aligned}
$$

iii. Zur besseren Unterscheidung von der Gleichheit im üblichen Sinne verwenden wir bei Gleichheit von formalen Potenzreihen das Zeichen $\doteq$. 
In der von D. H. Lehmer 22] eingeführten Form lautet die Riemann-Siegel-Formel dann

$$
Z(t) \sim 2 \sum_{n=1}^{N} \frac{\cos (\vartheta(t)-t \log n)}{\sqrt{n}}+\frac{(-1)^{N-1}}{\sqrt{a}} \sum_{n=0}^{\infty} \frac{C_{n}(z)}{a^{n}} \quad \text { für } t \rightarrow+\infty .
$$

Dabei ist

$$
\left.a:=\sqrt{\frac{t}{2 \pi}}, \quad N:=\lfloor a\rfloor 4\right), \quad z:=1-2(a-N)
$$

und

$$
C_{n}(z):=\frac{1}{2^{2 n}} \sum_{k=0}^{\lfloor 3 n / 4\rfloor} \frac{d_{k}^{(n)}}{\pi^{2 n-2 k}(3 n-4 k) !} F^{(3 n-4 k)}(z) \quad(n \geq 0)
$$

mit

$$
F(z):=\frac{\cos \frac{\pi}{2}\left(z^{2}+\frac{3}{4}\right)}{\cos \pi z}
$$

Die $d_{k}^{(n)}$ sind positive rationale Zahlen, die der Rekursionsformel

$$
\begin{array}{ll}
d_{k}^{(n+1)}=(3 n+1-4 k)(3 n+2-4 k) d_{k}^{(n)}+d_{k-1}^{(n)} & \left(n \geq 0,0 \leq k<\frac{3(n+1)}{4}\right), \\
d_{k}^{(n)}=0 & \text { für } k<0 \text { oder } k>\frac{3 n}{4}, \\
d_{3 n}^{(4 n)}=\lambda_{n} & (n \geq 0)
\end{array}
$$

genügen. Mit den Eulerschen Zahlen $E_{2 k}$ berechnen sich die $\lambda_{n}$ rekursiv aus

$$
\begin{aligned}
\lambda_{0} & =1 \\
(n+1) \lambda_{n+1} & =\sum_{k=0}^{n} 2^{4 k+1}\left|E_{2 k+2}\right| \lambda_{n-k} \quad(n \geq 0) .
\end{aligned}
$$

Die Funktion $\vartheta(t)$ besitzt die asymptotische Entwicklung

$$
\vartheta(t) \sim \frac{t}{2} \log \frac{t}{2 \pi}-\frac{t}{2}-\frac{\pi}{8}+\sum_{n=1}^{\infty} \frac{\left(2^{2 n-1}-1\right)\left|B_{2 n}\right|}{2^{2 n}(2 n-1) 2 n t^{2 n-1}} \quad \text { für } t \rightarrow+\infty .
$$

Dabei sind die $B_{2 n}$ die Bernoullischen Zahlen. Für das Restglied $R \vartheta(t)$ in

$$
\vartheta(t)=\frac{t}{2} \log \frac{t}{2 \pi}-\frac{t}{2}-\frac{\pi}{8}+\frac{1}{48 t}+\frac{7}{5760 t^{3}}+\frac{31}{80640 t^{5}}+R \vartheta(t)
$$

gilt für $t \geq 10$ die optimale Abschätzung

$$
|R \vartheta(t)|<\frac{1}{3322 t^{7}}
$$

\footnotetext{
4) $\lfloor a\rfloor$ ist die größte ganze Zahl $\leq a$.

5) $F(z)$ ist eine ganze Funktion der komplexen Veränderlichen $z$.
} 
Für die Restglieder $R_{K}(t)$ in der Darstellung ( $a, N$ und $z$ wie oben)

$$
Z(t)=2 \sum_{n=1}^{N} \frac{\cos (\vartheta(t)-t \log n)}{\sqrt{n}}+\frac{(-1)^{N-1}}{\sqrt{a}} \sum_{n=0}^{K} \frac{C_{n}(z)}{a^{n}}+R_{K}(t) \quad(K \geq 0)
$$

ist

$$
R_{K}(t)=O\left(t^{-\frac{2 K+3}{4}}\right) \text { für } K \geq 0 \text { und } t \rightarrow+\infty .
$$

Sie genügen für $t \geq 200$ und $K \leq 10$ den expliziten Abschätzungen

$$
\begin{array}{rlrl}
\left|R_{0}(t)\right|<0.127 t^{-3 / 4}, & \left|R_{1}(t)\right|<0.053 t^{-5 / 4}, & & \left|R_{6}(t)\right|<0.661 t^{-15 / 4}, \\
& \left|R_{2}(t)\right|<0.011 t^{-7 / 4}, & & \left|R_{7}(t)\right|<9.2 t^{-17 / 4}, \\
& \left|R_{3}(t)\right|<0.031 t^{-9 / 4}, & & \left|R_{8}(t)\right|<130 t^{-19 / 4}, \\
& \left|R_{4}(t)\right|<0.017 t^{-11 / 4}, & & \left|R_{9}(t)\right|<1837 t^{-21 / 4} \\
& \left|R_{5}(t)\right|<0.061 t^{-13 / 4}, & \left|R_{10}(t)\right|<25966 t^{-23 / 4} .
\end{array}
$$

Für $K \leq 4$ sind diese Abschätzungen optimal.

Die Riemann-Siegel-Formel (1] haben Riemann, Siegel [31, 32], Edwards [16. Ch. 7] und Crary [15] mit Hilfe der Sattelpunktmethode aus dem bekannten Schleifenintegral

$$
\zeta(s)=\frac{i}{2 \pi} \Gamma(1-s) \int_{C} \frac{(-x)^{s-1}}{e^{x}-1} d x
$$

hergeleitet, in dem der Integrationsweg $C$ eine Kurve ist, die im 1. Quadranten von $\infty$ kommt, die Singularität des Integranden bei $x=0$ umschlingt und im 4. Quadranten ${ }^{6)}$ hach $\infty$ geht (die Pole des Integranden bei $x= \pm 2 k \pi i(k \geq 1)$ werden nicht mit umschlungen). Eine besonders ausführliche Darstellung dieser Art der Herleitung der Riemann-Siegel-Formel gibt Edwards [16. Ch. 7]. Die Herleitung der Riemann-Siegel-Formel läßt sich jedoch erheblich vereinfachen, wenn man statt (9) die für reelle $t$ gültige Integraldarstellung

$$
Z(t)=2 \Re\left(e^{-i \vartheta(t)} \int_{0 \searrow 1} \frac{e^{-i \pi x^{2}} x^{-1 / 2+i t}}{e^{i \pi x}-e^{-i \pi x}} d x\right)
$$

verwendet, die sich aus der sogenannten Riemann-Siegel-Integralformel (Satz 4.1.6. S. 65] ergibt. Dabei ist der Integrationsweg eine von links oben nach rechts unten orientierte Gerade von der Steigung -1, die die reelle Achse zwischen den Punkten 0 und 1 trifft. Durch Anwendung der Sattelpunktmethode auf diese Integraldarstellung von $Z(t)$ haben wir in Kapitel 1, S. 7 die Riemann-Siegel-Formel hergeleitet; allerdings, um die dabei auftretenden Formeln möglichst einfach zu gestalten, in einer etwas anderen Form als in (1) angegeben. In Satz 2.1.6. S. 30 transformieren wir diese dann auf die Lehmersche Form (1).

\footnotetext{
6) In der Originalarbeit steht hier fälschlicherweise „2. Quadranten“.
} 
Die Darstellung (2) der Koeffizienten $C_{n}(z)$ als Kombination von Ableitungen der Funktion $F(z)$ ist zusammen mit der Rekursionsformel (3) bereits in der Zeitschrift Math. of Comp. 31, No. 139, July 1977, P. 803 in Form einer Mitteilung veröffentlicht worden. Der zugehörige, bis jetzt noch ausstehende Beweis, der übrigens ziemlich aufwendig ist, findet sich in Abschnitt 2.1. S. 19. Dort sind auch numerische Werte der Zahlen $\lambda_{n}$ für $n \leq 6$ angegeben. Vermutlich sind alle $\lambda_{n}$ und damit auch alle $d_{k}^{(n)}$ nicht nur positive rationale, sondern sogar natürliche Zahlen. Ein Beweis dafür ist dem Autor dieser Arbeit allerdings nicht bekannt.7)

Die Zahlen $d_{k}^{(n)}$ sind für $0 \leq n \leq 12$ in Form einer Primfaktorzerlegung in Tabelle II, S. 95 und die sich daraus ergebende exakte Darstellung der Koeffizienten $C_{n}(z)$ nach $(2)$ - die dabei auftretenden rationalen Zahlen $d_{k}^{(n)} /\left[2^{2 n}(3 n-4 k)\right.$ ! $]$ soweit wie möglich gekürzt - in Tabelle III, S. 97 aufgeführt. Für $n \leq 4$ findet man die $C_{n}(z)$ auch bei Haselgrove [20] und - gering abgewandelt - bei Edwards [16. S. 154], sowie für $n \leq 8$ bei Crary und Rosser [15].

Um die numerische Berechnung der $C_{n}(z)$ für $0 \leq n \leq 10 \mathrm{zu}$ erleichtern, sind die Koeffizienten ihrer Potenzreihenentwicklungen um den Punkt $z=0$ in Tabelle IV, S. 101 und die Koeffizienten ihrer Entwicklungen nach Tschebyscheffschen Polynomen 1. Art in Tabelle V, S. 113 auf 50 Dezimalstellen genau wiedergegeben. Die dazu notwendigen Rechnungen sind vom Autor auf der UNIVAC 1108 der Gesellschaft für Wissenschaftliche Datenverarbeitung Göttingen durchgeführt worden (vgl. Abschnitt 2.2. S. 31). Auf etwa 11 bis 20 Dezimalstellen genau finden sich diese Potenzreihenkoeffizienten für $n \leq 4$ auch bei Haselgrove [20] bzw. [16. S. 158] und für $n \leq 6$ auf 70 Dezimalstellen genau bei Crary und Rosser [15]. Die Potenzreihenkoeffizienten von $C_{7}(z)$ bis $C_{10}(z)$ und die für numerische Zwecke besonders gut geeigneten Tschebyscheffentwicklungen aus Tabelle $\mathrm{V}$ dürften neu sein.

Die asymptotische Entwicklung von $\vartheta(t)$, die schon bei Siegel [31, 32] zu finden ist, leiten wir in Abschnitt 4.2. S. 69 auf einem neuen Wege her. Für das Restglied $R \vartheta(t)$ in der Darstellung (4) ergibt sich dann auf relative einfache Weise die explizite Abschätzung (5). Damit ist gewährleistet, daß die Funktion $\vartheta(t)$, die zur numerischen Auswertung der Riemann-Siegel-Formel benötigt wird, mit Hilfe von (4) sehr genau berechnet werden kann.

Das wichtigste Ergebnis dieser Arbeit sind die expliziten Restabschätzungen (8), die wir unter Verwendung von optimalen Abschätzungen der Koeffizienten $C_{n}(z)(0 \leq n \leq 10,-1 \leq z \leq 1)$, die sich aus ihren Potenzreihenentwicklungen ergeben, in Abschnitt 3.2. S. 53 hergeleitet haben. Entsprechend schlechtere Abschätzungen von $R_{K}(t)(0 \leq K \leq 9)$, die ohne diese Potenzreihenentwicklungen gewonnen wurden, sind dort ebenfalls angegeben. Bisher sind Abschätzungen dieser Art nur für $R_{0}(t)$ und $R_{2}(t)$ bekannt. Die erste stammt von Titchmarsh [33] und [34. S. 331] aus dem Jahre 1935

$$
\left|R_{0}(t)\right|<1.50\left(\frac{t}{2 \pi}\right)^{-3 / 4} \quad \text { für } t>250 \pi,
$$

die zweite von Rosser, Yohe und Schoenfeld [30] aus dem Jahre 1968

$$
\left|R_{2}(t)\right|<2.88\left(\frac{t}{2 \pi}\right)^{-7 / 4} \quad \text { für } t>4000 \pi,
$$

\footnotetext{
7) Diese Vermutung konnte kürzlich von Arias de Reyna [2] bewiesen werden.
} 
deren Beweis allerdings bis heute nicht erschienen ist ${ }^{8)}$

Die Abschätzungen (8) zeigen, daß man $Z(t)$ aus (6) mit sehr hoher Genauigkeit berechnen kann. Alle bis heute mit Hilfe der Riemann-Siegel-Formel durchgeführten Berechnungen von $Z(t)$ stellen sich daher nachträglich als gerechtfertigt heraus.

Eine in diesem Zusammenhang interessante Frage ist, ob die Riemann-SiegelFormel in ihrer einfachsten Form (es ist $C_{0}(z)=F(z)$ )

$$
Z(t)=2 \sum_{n=1}^{N} \frac{\cos (\vartheta(t)-t \log n)}{\sqrt{n}}+\frac{(-1)^{N-1}}{\sqrt{a}} \cdot \frac{\cos \frac{\pi}{2}\left(z^{2}+\frac{3}{4}\right)}{\cos \pi z}+R_{0}(t)
$$

für die Bestimmung von sign $Z(t)^{9)}$ ұur numerischen Überprüfung der Riemannschen Vermutung immer ausreicht. Die Abschätzung (10) des Restgliedes $R_{0}(t)$ von Titchmarsh genügt dafür jedenfalls nicht immer, wie die Berechnungen von Lehmer [23], referenziert in [16. S. 175 ff.] und Rosser [30] zeigen. Die in dieser Arbeit bewiesene optimale Abschätzung $\left|R_{0}(t)\right|<0.127 t^{-3 / 4}(t \geq 200)$ ist jedoch für den bis heute untersuchten Bereich - etwa $t<2 \cdot 10^{6}$ - immer ausreichend 10$)$ Hs ist aber zu vermuten, daß das für hinreichend große $t$ nicht mehr der Fall ist, so daß zum Nachweis eines Vorzeichenwechsels von $Z(t) \mathrm{u}$. U. höhere Glieder der RiemannSiegel-Formel berücksichtigt werden müssen. Weitere Einzelheiten zu den bei der numerischen Überprüfung der Riemannschen Vermutung mit Hilfe der RiemannSiegel-Formel auftretenden Fragen findet der Leser bei Edwards [16, Ch. 8].

Die bereits von Siegel [31, 32] gefundene Groß-O-Abschätzung (7) der Restglieder $R_{K}(t)$, die wir in Abschnitt 3.2 beweisen, zeigt, daß sich die Riemann-SiegelFormel trotz der von $t$ abhängigen diskreten Veränderlichen $N$ im wesentlichen wie eine gewöhnliche asymptotische Entwicklung verhält. Ungeklärt ist nach wie vor, ob die Riemann-Siegel-Formel in der Form (1), was sehr wahrscheinlich ist, für jedes feste $t>0$ divergiert. Am Ende von Abschnitt 3.2 zeigen wir, daß das zumindest für spezielle Werte von $t$, nämlich für $t=t_{M}=2 \pi M^{2}(M$ ganz $>0)$, zutreffend ist 11 )

An dieser Stelle möchte ich Herrn Prof. Dr. M. Deuring danken, der durch sein freundliches Entgegenkommen das Erscheinen der vorliegenden Arbeit ermöglicht hat. Ferner danke ich Herrn Prof. Dr. H. L. de Vries für die auf der UNIVAC 1108 zur Verfügung gestellte Rechenzeit.

\footnotetext{
8) $\mathrm{Zu}$ diesen Abschätzungen vergleiche auch [16. S. 162 ff.]

9) Die Berechnung dieses Vorzeichens erfordert immer eine Auswertung des asymptotischen Teils der Riemann-Siegel-Formel - zumindest also die Berücksichtigung des $C_{0}$-Terms -, da die Hauptsumme $2 \sum_{n=1}^{N} \cos (\vartheta(t)-t \log n) / \sqrt{n}$ für die korrekte Beschreibung der Nullstellenverteilung von $Z(t)$ allein nicht ausreichend ist. So hat die Hauptsumme beispielsweise zwei konjugiert komplexe Nullstellen in der Nähe des Punktes $t=221.08$, während $Z(t)$ dort zwei eng benachbarte einfache reelle Nullstellen besitzt.

10) Das ist der Stand von 1979. Der vollständig untersuchte Bereich liegt inzwischen etwas unterhalb von $2.45 \cdot 10^{12}$ (Berechnungen von Gourdon und Demichel [19] aus dem Jahre 2004 unter Verwendung des Odlyzko-Schönhage-Algorithmus [28] zur optimierten Berechnung der Hauptsumme $\left.2 \sum_{n=1}^{N} \cos (\vartheta(t)-t \log n) / \sqrt{n}\right)$; jedoch sind nach Wissen des Autors noch immer keine Stellen bekannt, an denen die angegebene Abschätzung von $R_{0}(t)$ zur Vorzeichenbestimmung von $Z(t)$ nicht ausreichend wäre.

11) Berry [6] hat diese Vermutung 1995 für beliebige $t$ bewiesen.
} 



\section{Kapitel 1}

\section{Formale Herleitung der Riemann-Siegel-Formel}

\subsection{Definition und Integraldarstellung von $Z(t)$}

Es sei $t$ reell. Wir setzen

$$
\vartheta(t):=\Im \log \Gamma\left(\frac{1}{4}+i \frac{t}{2}\right)-\frac{t}{2} \log \pi
$$

und

$$
Z(t)=e^{i \vartheta(t)} \zeta\left(\frac{1}{2}+i t\right)
$$

Dabei ist der Logarithmus in 1.1 so zu bestimmen, daß $\vartheta(t)$ bei $t=0$ verschwindet.

Die in 1.2 eingeführte Funktion $Z(t)$ ergibt sich auf natürliche Weise aus der Riemann-Siegel-Integralformel (Satz 4.1.6, S. 65. Setzen wir nämlich dort $s=$ $1 / 2+i t$, so werden die beiden Ausdrücke auf der rechten Seite konjugiert komplex. Daher genügt es, wenn wir von einem dieser Ausdrücke den doppelten Realteil nehmen. Wir erhalten so

$$
\begin{gathered}
\pi^{-\frac{1}{4}-i \frac{t}{2}} \Gamma\left(\frac{1}{4}+i \frac{t}{2}\right) \zeta\left(\frac{1}{2}+i t\right) \\
=2 \Re\left(\pi^{-\frac{1}{4}+i \frac{t}{2}} \Gamma\left(\frac{1}{4}-i \frac{t}{2}\right) \int_{0 \searrow 1} \frac{e^{-i \pi x^{2}} x^{-1 / 2+i t}}{e^{i \pi x}-e^{-i \pi x}} d x\right) .
\end{gathered}
$$

\footnotetext{
1) Diese heute übliche Bezeichnung für die rechtsstehende Funktion geht auf Titchmarsh zurück, der sie 1951 in seinem Buch über die Zetafunktion [34. S. 79] erstmals verwendet hat. Es handelt sich dabei wohl nicht um ein lateinisches "Z", sondern um ein großes griechisches Zeta, so daß die Funktion verbal mit „Groß-Zeta von t" bezeichnet werden sollte. Sie wird in der Literatur oft „Hardys-Z-Funktion“ genannt, was ein wenig mißverständlich ist, da sie in G. H. Hardys Publikationen nicht explizit vorkommt und insbesondere die Bezeichnung $Z(t)$ dort nicht zu finden ist.
} 
Wegen 1.1 gilt die Zerlegung (von allen Logarithmen ist der Hauptwert zu nehmen)

$$
\begin{aligned}
\pi^{-\frac{1}{4}-i \frac{t}{2}} \Gamma\left(\frac{1}{4}+i \frac{t}{2}\right) & =e^{\Re \log \Gamma\left(\frac{1}{4}+i \frac{t}{2}\right)-\frac{1}{4} \log \pi} \cdot e^{i\left(\Im \log \Gamma\left(\frac{1}{4}+i \frac{t}{2}\right)-\frac{t}{2} \log \pi\right)} \\
& =f(t) \cdot e^{i \vartheta(t)},
\end{aligned}
$$

wobei wir zur Abkürzung

$$
f(t):=e^{\Re \log \Gamma\left(\frac{1}{4}+i \frac{t}{2}\right)-\frac{1}{4} \log \pi}
$$

gesetzt haben. Da $f(t)$ und $\vartheta(t)$ reell sind, erhält man hieraus durch Übergang zum konjugiert Komplexen

$$
\pi^{-\frac{1}{4}+i \frac{t}{2}} \Gamma\left(\frac{1}{4}-i \frac{t}{2}\right)=f(t) \cdot e^{-i \vartheta(t)} .
$$

Damit folgt aus 1.2 und 1.3 nach Kürzen von $f(t)$ die Integraldarstellung

$$
Z(t)=2 \Re\left(e^{-i \vartheta(t)} \int_{0 \searrow 1} \frac{e^{-i \pi x^{2}} x^{-1 / 2+i t}}{e^{i \pi x}-e^{-i \pi x}} d x\right),
$$

aus der unmittelbar hervorgeht, daß $Z(t)$ reell ist. Wegen $|Z(t)|=|\zeta(1 / 2+i t)|$ läßt sich die Untersuchung der Nullstellen der Zetafunktion auf der kritischen Geraden $\Re(s)=1 / 2$ durch Einführung der Funktion $Z(t)$ auf ein reelles Problem zurückführen.

Die Integraldarstellung (1.4) kann auf einfache Weise verallgemeinert werden. Es sei $N$ eine natürliche Zahl. Verschieben wir den Integrationsweg $0 \searrow 1$ um $N$ nach rechts, so ergibt sich mit Hilfe des Residuensatzes

$$
\begin{aligned}
Z(t)= & 2 \Re\left(e^{-i \vartheta(t)} \int_{N \searrow N+1} \frac{e^{-i \pi x^{2}} x^{-1 / 2+i t}}{e^{i \pi x}-e^{-i \pi x}} d x\right) \\
& +2 \Re\left(2 \pi i e^{-i \vartheta(t)} \sum_{n=1}^{N} \operatorname{Res}_{x=n} \frac{e^{-i \pi x^{2}} x^{-1 / 2+i t}}{e^{i \pi x}-e^{-i \pi x}}\right),
\end{aligned}
$$

und wir erhalten wegen

$$
\begin{aligned}
2 \pi i e^{-i \vartheta(t)} \sum_{n=1}^{N} \operatorname{Res}_{x=n} \frac{e^{-i \pi x^{2}} x^{-1 / 2+i t}}{e^{i \pi x}-e^{-i \pi x}} & =\sum_{n=1}^{N} 2 \pi i e^{-i \vartheta(t)} e^{-i \pi n^{2}} n^{-1 / 2+i t} \operatorname{Res}_{x=n} \frac{1}{2 i \sin \pi x} \\
& =\sum_{n=1}^{N} 2 \pi i e^{i(t \log n-\vartheta(t))} n^{-1 / 2}(-1)^{n^{2}} \frac{(-1)^{n}}{2 \pi i} \\
& =\sum_{n=1}^{N} \frac{e^{i(t \log n-\vartheta(t))}}{\sqrt{n}}
\end{aligned}
$$

die für jede natürliche Zahl $N$ geltende Integraldarstellung

$$
Z(t)=2 \sum_{n=1}^{N} \frac{\cos (\vartheta(t)-t \log n)}{\sqrt{n}}+\Re I_{N}(t)
$$


mit

$$
I_{N}(t)=2 e^{-i \vartheta(t)} \int_{N \searrow N+1} \frac{e^{-i \pi x^{2}} x^{-1 / 2+i t}}{e^{i \pi x}-e^{-i \pi x}} d x .
$$

Der Integrationsweg $N \searrow N+1$ ist entsprechend dem Integrationsweg $0 \searrow 1 \mathrm{zu}$ verstehen (vgl. Abschnitt 4.1, S. 61 ff.). Wenn man leere Summen Null setzt, bleibt 1.5 auch noch für $N=0$ richtig und man erhält die Gleichung 1.4 zurück.

Wir bemerken noch, daß man sich bei der Untersuchung von $Z(t)$ für große $|t|$ auf positive $t$ beschränken kann, da $Z(t)$ eine gerade Funktion ist. Wegen $\Gamma(\bar{s})=\overline{\Gamma(s)}$ ist nämlich $\vartheta(-t)=-\vartheta(t)$ und daher nach 1.2

$$
Z(-t)=e^{-i \vartheta(t)} \zeta\left(\frac{1}{2}-i t\right)=\overline{e^{i \vartheta(t)} \zeta\left(\frac{1}{2}+i t\right)}=\overline{Z(t)}=Z(t),
$$

denn $Z(t)$ ist reell.

\subsection{Anwendung der Sattelpunktmethode}

Von jetzt ab sei $t>0$. Wir wollen das Integral des Ausdruckes $I_{N}(t)$ in 1.6 nach der Sattelpunktmethode ${ }^{2)}$ auswerten. Wegen der Periodizität des Nenners $e^{i \pi x}-e^{-i \pi x}=2 i \sin \pi x$ vernachlässigen wir diesen und betrachten nur den Zähler $e^{-i \pi x^{2}} x^{-1 / 2+i t}=e^{-i \pi x^{2}+(-1 / 2+i t) \log x}$. Wir haben also die Sattelpunkte von $\Re\left[-i \pi x^{2}+(-1 / 2+i t) \log x\right]$ zu bestimmen. Bekanntlich sind das die Punkte, an denen die erste Ableitung von $-i \pi x^{2}+(-1 / 2+i t) \log x$, also $-2 \pi i x+(-1 / 2+i t) / x$, verschwindet. Das ist für

$$
x= \pm \sqrt{\frac{t}{2 \pi}-\frac{1}{4 \pi i}} \sim \pm \sqrt{\frac{t}{2 \pi}} \quad(t \rightarrow+\infty)
$$

der Fall. Die gesuchten Sattelpunkte liegen daher für große positive $t$ in der Nähe der Punkte $x=\sqrt{t /(2 \pi)}$ und $x=-\sqrt{t /(2 \pi)}$. Wegen des Verzweigungspunktes des Integranden bei $x=0$ ist für uns nur der Sattelpunkt bei $x=\sqrt{t /(2 \pi)}$ brauchbar. Wir setzen daher

$$
a:=\sqrt{\frac{t}{2 \pi}}
$$

und haben den Integrationsweg in (1.6) durch diesen Punkt zu legen. Wenn wir dort $N:=\lfloor a\rfloor{ }^{3)}$ wählen und $a$ keine ganze Zahl ist, läßt sich das wegen $N<a<N+1$ erreichen. Der Integrationsweg ist in diesem Fall also die Gerade von der Steigung -1 , von links oben nach rechts unten orientiert, die die reelle Achse im Punkte $x=a$ schneidet. Für ganzes $a$ setzen wir wieder $N:=\lfloor a\rfloor=a$ und nehmen denselben Integrationsweg wie eben. Den Pol des Integranden bei $x=a$ umgehen wir dabei mit einem kleinen Halbkreis, und zwar so, daß der Pol - unter Berücksichtigung der Orientierung - rechts des Integrationsweges liegt, so daß der

2) Allgemeines zur Sattelpunktmethode - auch Methode des stärksten Abstiegs - findet man z. B. in [13. S. 455-460], [14. S. 526-532] oder 35. S. 235 ff.]. Riemann hat sie Ende der 1850-er Jahre zur Herleitung der Riemann-Siegel-Formel wohl als erster angewendet (vgl. Siegels Arbeit 31. 32] von 1932 über Riemanns Nachlaß).

3) Wie üblich bezeichnet $\lfloor a\rfloor$ die größte ganze Zahl $\leq a$. 
Integrationsweg auch hier die reelle Achse zwischen den Punkten $N$ und $N+1$ schneidet. Für die so in den beiden zu unterscheidenden Fällen - $a$ ganz bzw. nicht ganz - festgelegten Integrationswege führen wir das gemeinsame Zeichen $y_{a}^{4}$ ein. Aus 1.5 und 1.6 folgt damit

$$
\begin{gathered}
Z(t)=2 \sum_{n=1}^{N} \frac{\cos (\vartheta(t)-t \log n)}{\sqrt{n}}+\Re I_{N}(t), \\
I_{N}(t)=2 e^{-i \vartheta(t)} \int \frac{e^{-i \pi x^{2}} x^{-1 / 2+i t}}{e^{i \pi x}-e^{-i \pi x}} d x, \\
\searrow_{a} \\
a:=\sqrt{\frac{t}{2 \pi}}, \quad N:=\lfloor a\rfloor .
\end{gathered}
$$

Es sei darauf hingewiesen, daß der Integrationsweg bei strenger Anwendung der Sattelpunktmethode - zumindest in der Nähe des Sattelpunktes - mit dem Weg des stärksten Abstiegs zusammenfallen muß. Wegen des Faktors $e^{-i \pi x^{2}}$ geht aber der Betrag des Integranden in (1.7) exponentiell gegen Null, wenn sich $x$ auf dem Integrationsweg $\searrow_{a}$ von der reellen Achse entfernt, und daher besteht kein Anlaß, diesen besonders einfach zu handhabenden Integrationsweg zu ändern. Ferner sei noch angemerkt, daß sich weder das Ersetzen des exakten Sattelpunktes mit dem benachbarten Punkt $a$ noch die Abänderung des Integrationsweges für ganzzahliges $a$ bei der Restabschätzung in Kapitel 3. S. 41 negativ auswirken werden.

Da der Logarithmus in $x^{-1 / 2+i t}=e^{(-1 / 2+i t) \log x}$ die eindeutige holomorphe Fortsetzung der reellen Logarithmusfunktion in die längs der negativen reellen Achse bis zum Nullpunkt aufgeschnittenen $x$-Ebene ist ${ }^{5)}$ können wir den Zähler des Integranden in (1.7) durch Entwicklung um den Punkt $x=a$ wie folgt umformen

$$
\begin{aligned}
e^{-i \pi x^{2}} x^{-1 / 2+i t} & =e^{-i \pi(x-a+a)^{2}+(-1 / 2+i t) \log (x-a+a)} \\
& =e^{(-1 / 2+i t) \log a-i \pi a^{2}} \cdot e^{-2 \pi i(x-a)^{2}} \widetilde{g}(a, x-a),
\end{aligned}
$$

mit der Abkürzung

$$
\widetilde{g}(a, z):=\exp \left[\left(-\frac{1}{2}+i t\right) \log \left(1+\frac{z}{a}\right)-2 \pi i a z+i \pi z^{2}\right] .
$$

Wegen $a>0$ ist $\widetilde{g}(a, z)$ daher in der längs der negativen reellen Achse bis zum Punkt $z=-a$ aufgeschnittenen $z$-Ebene eine eindeutige holomorphe Funktion von $z$ mit $\widetilde{g}(a, 0) \equiv 1$.

Unter Berücksichtigung von

$$
e^{(-1 / 2+i t) \log a-i \pi a^{2}}=\left(\frac{t}{2 \pi}\right)^{-\frac{1}{4}} e^{i\left(\frac{t}{2} \log \frac{t}{2 \pi}-\frac{t}{2}\right)}
$$

folgt damit aus 1.7

$$
I_{N}(t)=2\left(\frac{t}{2 \pi}\right)^{-\frac{1}{4}} e^{i\left(\frac{t}{2} \log \frac{t}{2 \pi}-\frac{t}{2}-\vartheta(t)\right)} \int \frac{e^{-2 \pi i(x-a)^{2}}}{e^{i \pi x}-e^{-i \pi x}} \widetilde{g}(a, x-a) d x
$$

4) Der Punkt bezeichnet die Lage des Pols relativ zum Integrationsweg bei ganzzahligem $a$.

5) Das ergibt sich aus dem Beweis der Riemann-Siegel-Integralformel in Satz 4.1.6, S. 65. 
und wegen $e^{i \pi x}-e^{-i \pi x}=2 i \sin \pi x$ läßt sich das auch so schreiben:

$$
\begin{gathered}
I_{N}(t)=(-1)^{N-1}\left(\frac{t}{2 \pi}\right)^{-\frac{1}{4}} U \cdot S, \\
U:=\exp i\left(\frac{t}{2} \log \frac{t}{2 \pi}-\frac{t}{2}-\frac{\pi}{8}-\vartheta(t)\right)^{6)} \\
S:=(-1)^{N-1} e^{i \pi / 8} \int \frac{e^{-2 \pi i(x-a)^{2}}}{i \sin \pi x} \widetilde{g}(a, x-a) d x . \\
\searrow_{a}
\end{gathered}
$$

Den Ausdruck $S$ formen wir noch in geeigneter Weise um. Wir substituieren im Integral mit $x=i v /(2 \sqrt{\pi})+N+1 / 2$ und führen die Größen

$$
\tau:=\frac{1}{2 \sqrt{2 t}}, \quad q:=\sqrt{\pi}[1-2(a-N)]
$$

ein. Damit wird

$$
t=\frac{1}{8 \tau^{2}}, \quad a=\frac{1}{4 \sqrt{\pi} \tau}, \quad \quad N+\frac{1}{2}-a=\frac{q}{2 \sqrt{\pi}},
$$

und wegen $v=2 i \sqrt{\pi}(N+1 / 2-x)$ transformiert sich der Integrationsweg $\searrow_{a}$ bezüglich $x$ in ${ }_{i q} \dot{\swarrow}$ bezüglich $v$. Beachtet man noch

$$
\begin{gathered}
x-a=i \frac{v}{2 \sqrt{\pi}}+N+\frac{1}{2}-a=i \frac{v-i q}{2 \sqrt{\pi}}, \\
-2 \pi i(x-a)^{2}=\frac{i}{2}(v-i q)^{2}=i \frac{v^{2}}{2}+q v-i \frac{q^{2}}{2}, \\
\sin \pi x=\cos \pi\left(i \frac{v}{2 \sqrt{\pi}}+N\right)=(-1)^{N} \cosh \frac{\sqrt{\pi}}{2} v,
\end{gathered}
$$

so erhalten wir nach Kürzen von $(-1)^{N}$ und Umkehrung der Orientierung des Integrationsweges

$$
S=\frac{1}{2 \sqrt{\pi}} e^{i \pi / 8-i q^{2} / 2} \int_{\nearrow^{i q}} \frac{e^{i v^{2} / 2+q v}}{\cosh \frac{\sqrt{\pi}}{2} v} \widetilde{g}\left(\frac{1}{4 \sqrt{\pi} \tau}, i \frac{v-i q}{2 \sqrt{\pi}}\right) d v .
$$

Aus 1.7 und 1.9 folgt dann, wenn wir noch einmal alles zusammenfassen und zur Vereinfachung

$$
g(\tau, z):=\widetilde{g}\left(\frac{1}{4 \sqrt{\pi} \tau}, i \frac{z}{2 \sqrt{\pi}}\right)
$$

setzen:

6) Die Einführung dieses Ausdrucks ergibt sich auf natürliche Weise aus der asymptotischen Entwicklung von $\vartheta(t)($ Satz 4.2 .3 , S. 71 . Somit ist $U \sim 1$ für $t \rightarrow+\infty$. 
Satz 1.2.1 (Darstellung von $Z(t)$ nach Anwendung der Sattelpunktmethode).

$$
Z(t)=2 \sum_{n=1}^{N} \frac{\cos (\vartheta(t)-t \log n)}{\sqrt{n}}+(-1)^{N-1}\left(\frac{t}{2 \pi}\right)^{-\frac{1}{4}} \Re(U \cdot S)
$$

mit

$$
\begin{gathered}
a:=\sqrt{\frac{t}{2 \pi}}, \quad N:=\lfloor a\rfloor, \quad q:=\sqrt{\pi}[1-2(a-N)], \quad \tau:=\frac{1}{2 \sqrt{2 t}}, \\
U:=\exp i\left(\frac{t}{2} \log \frac{t}{2 \pi}-\frac{t}{2}-\frac{\pi}{8}-\vartheta(t)\right), \\
S:=\frac{1}{2 \sqrt{\pi}} e^{i \pi / 8-i q^{2} / 2} \int \frac{e^{i v^{2} / 2+q v}}{\cosh \frac{\sqrt{\pi}}{2} v} g(\tau, v-i q) d v \\
\gamma^{i q} \\
g(\tau, z):=\exp \left[\left(-\frac{1}{2}+\frac{i}{8 \tau^{2}}\right) \log (1+2 i \tau z)+\frac{z}{4 \tau}-i \frac{z^{2}}{4}\right] .
\end{gathered}
$$

Den rechtsstehenden Ausdruck für $g(\tau, z)$ erhält man aus der Definition dieser Funktion in 1.10, zusammen mit Gleichung (1.8). Wegen $\tau>0$ ist $g(\tau, z)$ daher in der längs der positiven imaginären Achse bis zum Punkt $z=i /(2 \tau)$ aufgeschnittenen $z$-Ebene eine eindeutige holomorphe Funktion von $z$ mit $g(\tau, 0) \equiv 1$.

Der Integrationsweg $\nearrow^{i q}$ ist jetzt die von links unten nach rechts oben orientierte Gerade der Steigung 1 durch den Punkt $v=i q$. Dabei ist für ganzzahliges $a$, wenn $q=\sqrt{\pi}$ wird, der Pol des Integranden bei $v=i \sqrt{\pi}$ mit einem kleinen Halbkreis so zu umgehen, daß der Pol - unter Berücksichtigung der Orientierung - links des Integrationsweges liegt, so daß der Integrationsweg auch hier die imaginäre Achse zwischen den Punkten $-i \sqrt{\pi}$ und $i \sqrt{\pi}$ schneidet.

Aus Satz 1.2.1 werden wir im nächsten Abschnitt die Riemann-Siegel-Formel herleiten. Um die dabei auftretenden Formeln möglichst einfach zu gestalten, haben wir dem Ausdruck $S$ die obenstehende Form gegeben und die Hilfsvariable $\tau$ eingeführt. Dabei erweist es sich als besonders vorteilhaft, daß die Funktion $g(\tau, z)$ im Gegensatz zu $\widetilde{g}(a, z)$ die Zahl $\pi$ nicht mehr enthält.

\subsection{Die Riemann-Siegel-Formel}

Wir wollen jetzt den Ausdruck $\Re(U \cdot S)$ aus Satz 1.2.1 in eine formale Reihe nach Potenzen von $\tau$ entwickeln. Wegen $\tau=1 /(2 \sqrt{2 t})$ entspricht das einer Entwicklung von $\Re(U \cdot S)$ nach negativen Potenzen von $\sqrt{t}$. Wir erhalten diese Entwicklung, wenn wir zunächst für $U$ bzw. $S$ je eine formale Reihe nach Potenzen von $\tau$ herleiten, diese Reihen miteinander multiplizieren und zum Realteil übergehen.

Der Ausdruck $U$ besitzt eine asymptotische Entwicklung der Form

$$
U \sim \sum_{n=0}^{\infty} i^{n} \alpha_{n} \tau^{2 n} \quad(\tau \rightarrow 0)
$$


Die Koeffizienten $\alpha_{n}$ sind rationale Zahlen mit $\alpha_{0}=1$. Alles weitere hierzu findet man in Satz 4.2.4. S. 74 .

Wir bestimmen jetzt das Verhalten von $S$ für $\tau \rightarrow 0$. Die Funktion $g(\tau, z)$ ist gerade so konstruiert, daß $\lim _{\tau \rightarrow 0} g(\tau, z) \equiv 1$ ist. Ersetzen wir nämlich den Logarithmus in

$$
\left(-\frac{1}{2}+\frac{i}{8 \tau^{2}}\right) \log (1+2 i \tau z)+\frac{z}{4 \tau}-i \frac{z^{2}}{4}
$$

mit den ersten beiden Gliedern seiner Potenzreihe, so wird dieser Ausdruck für jedes feste $z$ gleich

$$
\begin{gathered}
\left(-\frac{1}{2}+\frac{i}{8 \tau^{2}}\right)\left(2 i z \tau+2 z^{2} \tau^{2}+O\left(\tau^{3}\right)\right)+\frac{z}{4 \tau}-i \frac{z^{2}}{4} \\
=-\frac{z}{4 \tau}+i \frac{z^{2}}{4}+O(\tau)+\frac{z}{4 \tau}-i \frac{z^{2}}{4}=O(\tau) .
\end{gathered}
$$

Folglich verschwindet 1.12 für $\tau \rightarrow 0$ und es ist wie behauptet $\lim _{\tau \rightarrow 0} g(\tau, z) \equiv 1$.

Nach Definition von $q$ in Satz 1.2 .1 gilt immer $-\sqrt{\pi}<q \leq \sqrt{\pi}$. Wir setzen für diese $q$

$$
\widetilde{F}(q):=\frac{1}{2 \sqrt{\pi}} e^{i \pi / 8-i q^{2} / 2} \int_{\nearrow^{i q}} \frac{e^{i v^{2} / 2+q v}}{\cosh \frac{\sqrt{\pi}}{2} v} d v
$$

und haben dann $S \sim \widetilde{F}(q)$ für $\tau \rightarrow 0$. Eine einfache Anwendung des Cauchyschen Integralsatzes zeigt, daß man den Integrationsweg $\nearrow^{i q}$ in 1.13 mit $i \sqrt{\pi} \nearrow-i \sqrt{\pi}$ ersetzen darf ohne den Wert des Integrals zu verändern, und zwar auch dann, wenn $a$ ganzzahlig und $q=\sqrt{\pi}$ wird. Nach Satz 4.1.2. S. 61 ist $\widetilde{F}(q)$ daher mit elementaren Funktionen darstellbar:

$$
\widetilde{F}(q)=\frac{\cos \left(\frac{q^{2}}{2}+\frac{3 \pi}{8}\right)}{\cos \sqrt{\pi} q}+i \frac{\sqrt{2} \cos \frac{\sqrt{\pi}}{2} q-\sin \left(\frac{q^{2}}{2}+\frac{3 \pi}{8}\right)}{\cos \sqrt{\pi} q}
$$

Setzen wir noch für reelle $q$

$$
\begin{aligned}
& \widehat{F}(q):=\Re \widetilde{F}(q)=\frac{\cos \left(\frac{q^{2}}{2}+\frac{3 \pi}{8}\right)}{\cos \sqrt{\pi} q}, \\
& \widehat{F}(q):=\Im \widetilde{F}(q)=\frac{\sqrt{2} \cos \frac{\sqrt{\pi}}{2} q-\sin \left(\frac{q^{2}}{2}+\frac{3 \pi}{8}\right)}{\cos \sqrt{\pi} q},
\end{aligned}
$$

so wird, da nach 1.11 $U \sim 1$ für $\tau \rightarrow 0$ gilt, $U \cdot S \sim \widetilde{F}(q)$ und $\Re(U \cdot S) \sim \widehat{F}(q)$ für $\tau \rightarrow 0$. Wegen $(t /(2 \pi))^{1 / 4}=\sqrt{a}$ folgt so aus Satz 1.2.1 für $Z(t)$ die Darstellung $(a$, $N$ und $q$ wie dort definiert)

$$
Z(t) \sim 2 \sum_{n=1}^{N} \frac{\cos (\vartheta(t)-t \log n)}{\sqrt{n}}+\frac{(-1)^{N-1}}{\sqrt{a}} \cdot \frac{\cos \left(\frac{q^{2}}{2}+\frac{3 \pi}{8}\right)}{\cos \sqrt{\pi} q} \quad(\tau \rightarrow 0) .
$$

\footnotetext{
7) Genau genommen ist diese Darstellung von $\widetilde{F}(q)$ die holomorphe Fortsetzung der in 1.13 für reelle $q$ mit $-\sqrt{\pi}<q \leq \sqrt{\pi}$ eingeführten Funktion $\widetilde{F}(q)$ in die ganze $q$-Ebene.
} 
Das ist die Riemann-Siegel-Formel in ihrer einfachsten Form.

Durch Entwicklung von $g(\tau, z)$ in eine Potenzreihe nach $\tau^{8)}$ läßt sich $1.16 \mathrm{zu}$ einer vollen asymptotischen Entwicklung ausbauen. Für $2 \tau|z|<1^{9)}$ hann man den Logarithmus in 1.12 mit der Reihe $\sum_{n=1}^{\infty}(-1)^{n-1}(2 i \tau z)^{n} / n$ ersetzen. Diese Reihe konvergiert für $2 \tau|z| \leq \theta<1(\theta$ reell $>0)$ absolut und gleichmäßig als Funktion der beiden Veränderlichen $\tau$ und $z$. Wir können daher den so entstehenden Ausdruck nach Potenzen von $\tau$ anordnen. Da 1.12 für $\tau \rightarrow 0$ verschwindet, erhalten wir auf diese Weise eine Potenzreihe in $\tau$, deren absolutes Glied verschwindet und deren Koeffizienten Polynome in $z$ sind. Setzen wir jetzt diese Potenzreihe in die Potenzreihe der Exponentialfunktion ein und ordnen wieder nach Potenzen von $\tau$, so ergibt sich die gesuchte Entwicklung von $g(\tau, z)$. Diese konvergiert ebenfalls für $2 \tau|z| \leq \theta<1$ absolut und gleichmäßig als Funktion der beiden Veränderlichen $\tau$ und $z$, ihr konstantes Glied ist 1 und ihre Koeffizienten sind wiederum Polynome in $z$. Wir haben daher in dem Ansatz mit unbestimmten Koeffizienten

$$
g(\tau, z)=\sum_{n=0}^{\infty} P_{n}(z) \tau^{n} \quad(2 \tau|z|<1)
$$

die Polynome $P_{n}(z)$ zu bestimmen. Wegen $g(0, z) \equiv 1 \equiv g(\tau, 0)$ müssen die $P_{n}(z)$ den Bedingungen

$$
\begin{aligned}
& P_{0}(z) \equiv 1, \\
& P_{n}(0)=0 \quad(n \geq 1)
\end{aligned}
$$

genügen.

Der Einfachheit halber bezeichnen wir die partielle Ableitung von $g(\tau, z)$ nach $z$ mit $g^{\prime}(\tau, z)$. Dann ist die logarithmische Ableitung von $g(\tau, z)$ nach $z$ gleich der Ableitung des Ausdruckes 1.12 nach $z$ und wir erhalten nach einer einfachen Rechnung

$$
\frac{g^{\prime}(\tau, z)}{g(\tau, z)}=\tau \frac{z^{2}-i}{1+2 i \tau z}
$$

und daraus die homogene Differentialgleichung erster Ordnung

$$
(1+2 i \tau z) g^{\prime}(\tau, z)-\tau\left(z^{2}-i\right) g(\tau, z)=0 .
$$

Wir tragen hier die Gleichung (1.17) ein:

$$
(1+2 i \tau z) \sum_{n=0}^{\infty} P_{n}^{\prime}(z) \tau^{n}-\left(z^{2}-i\right) \sum_{n=0}^{\infty} P_{n}(z) \tau^{n+1}=0 .
$$

\footnotetext{
8) Siegel [31. 32!, Edwards [16. Ch. 7.5] und Crary 15] entwickeln die der Funktion $g(\tau, z)$ entsprechenden Funktionen - bei Verwendung unserer Schreibweise - nicht nach $\tau$ sondern nach $z$. Die formale Herleitung der Riemann-Siegel-Formel führt zwar auch so zum Ziel, jedoch ist es der natürlichere Weg, wenn man zur Entwicklung von $\Re(U \cdot S)$ nach Potenzen von $\tau$ die Funktion $g(\tau, z)$ gleich in eine Potenzreihe nach $\tau$ entwickelt. Das erweist sich für die Restabschätzung in Kapitel 3 als wichtige Grundvoraussetzung; denn andernfalls, d. h. bei einer Entwicklung von $g(\tau, z)$ nach Potenzen von $z$, würden die dann auftretenden Formeln derart kompliziert, daß eine realistische Restabschätzung mit vertretbarem Aufwand nicht mehr durchführbar wäre.

9) Für uns ist $\tau$ immer positiv, so daß die Betragstriche bei $\tau$ fortfallen können.
} 
Da nach 1.18 $P_{0}^{\prime}(z)=0$ ist, können wir das in die Form

$$
\sum_{n=0}^{\infty} P_{n+1}^{\prime}(z) \tau^{n+1}+\sum_{n=0}^{\infty} 2 i z P_{n}^{\prime}(z) \tau^{n+1}-\sum_{n=0}^{\infty}\left(z^{2}-i\right) P_{n}(z) \tau^{n+1}=0
$$

bringen und durch Koeffizientenvergleich folgt

$$
P_{n+1}^{\prime}(z)+2 i z P_{n}^{\prime}(z)-\left(z^{2}-i\right) P_{n}(z)=0 \quad(n \geq 0) .
$$

Wir ersetzen $z$ mit $x$ und integrieren von 0 bis $z$ über $x$. Unter Beachtung von 1.18 erhalten wir

$$
P_{n+1}(z)+2 i \int_{0}^{z} x P_{n}^{\prime}(x) d x-\int_{0}^{z}\left(x^{2}-i\right) P_{n}(x) d x=0 \quad(n \geq 0) .
$$

Partielle Integration gestattet die Umformung

$$
\int_{0}^{z} x P_{n}^{\prime}(x) d x=z P_{n}(z)-\int_{0}^{z} P_{n}(x) d x
$$

und es ergibt sich die Rekursionsformel

$$
\begin{aligned}
P_{n+1}(z) & =\int_{0}^{z}\left(x^{2}+i\right) P_{n}(x) d x-2 i z P_{n}(z) \quad(n \geq 0), \\
P_{0}(z) & \equiv 1
\end{aligned}
$$

aus der man unmittelbar folgende Eigenschaften der Polynome $P_{n}(z)$ ablesen kann:

(a) $P_{n}(z)$ ist für gerades $n$ eine gerade und für ungerades $n$ eine ungerade Funktion von $z$.

(b) $P_{n}(z)$ hat den Grad $3 n$.

(c) Die Koeffizienten der Potenzen $z^{0}$ bis $z^{n-1}$ des Polynoms $P_{n}(z)$ verschwinden, so daß die Funktionen $z^{-n} P_{n}(z)$ Polynome vom Grade $2 n$ sind.

Um die Berechnung der Polynome $P_{n}(z)$ aus der Rekursionsformel $1.21 \mathrm{zu}$ vereinfachen, machen wir mit unbestimmten Koeffizienten $a_{k}^{(n)}$ den Ansatz

$$
P_{n}(z)=\sum_{k=0}^{n} i^{k-n} \frac{a_{k}^{(n)}}{(n+2 k) !} z^{n+2 k} \quad(n \geq 0),
$$

der aufgrund der Eigenschaften (a), (b) und (c) in dieser Form zulässig ist. Wir 
tragen das in 1.21 ein, definieren noch $a_{k}^{(n)}=0$ für $k<0$ oder $k>n$ und erhalten

$$
\begin{aligned}
& \sum_{k=0}^{n+1} i^{k-n-1} \frac{a_{k}^{(n+1)}}{(n+2 k+1) !} z^{n+2 k+1}= \sum_{k=0}^{n} i^{k-n} \frac{a_{k}^{(n)}}{(n+2 k) !} \cdot \frac{z^{n+2 k+3}}{n+2 k+3} \\
&+\sum_{k=0}^{n} i^{k-n+1} \frac{a_{k}^{(n)}}{(n+2 k) !} \cdot \frac{z^{n+2 k+1}}{n+2 k+1} \\
&-\sum_{k=0}^{n} i^{k-n+1} \frac{2 a_{k}^{(n)}}{(n+2 k) !} z^{n+2 k+1} \\
&=\sum_{k=0}^{n+1}\left(\frac{i^{k-n-1} a_{k-1}^{(n)}}{(n+2 k+1)(n+2 k-2) !}+\frac{i^{k-n+1} a_{k}^{(n)}}{(n+2 k+1) !}-\frac{2 i^{k-n+1} a_{k}^{(n)}}{(n+2 k) !}\right) z^{n+2 k+1} .
\end{aligned}
$$

Durch Koeffizientenvergleich folgt hieraus - nach Multiplikation mit $(n+2 k+1)$ ! und Kürzen von $i^{k-n-1}-$ die Rekursionsformel

$$
\begin{aligned}
& a_{k}^{(n+1)}=(n+2 k-1)(n+2 k) a_{k-1}^{(n)}+(2 n+4 k+1) a_{k}^{(n)} \\
& 0 \leq k \leq n+1 \\
& a_{0}^{(0)}=1, \\
& a_{k}^{(n)}=0 \quad \text { für } k<0 \text { oder } k>n,
\end{aligned}
$$

aus der unmittelbar hervorgeht, daß die $a_{k}^{(n)}$ natürliche Zahlen sind. Dabei ergibt sich die Anfangsbedingung $a_{0}^{(0)}=1$ wegen $P_{0}(z) \equiv 1$ direkt aus 1.22 .

Mit Hilfe dieser Rekursionsformel lassen sich die $a_{k}^{(n)}$ sehr leicht berechnen. Für $n \leq 11$ findet man ihre exakten Werte - aus Gründen der Übersichtlichkeit in Primfaktoren zerlegt - in Tabelle I, S. 91. Bei der Restabschätzung in Kapitel 3 werden uns diese numerischen Werte noch nützlich sein.

Wir ersetzen jetzt die Funktion $g(\tau, v-i q)$ in der Integraldarstellung von $S$ aus Satz 1.2 .1 mit der Potenzreihe $\sum_{n=0}^{\infty} P_{n}(v-i q) \tau^{n}$, vertauschen die Integration mit der Summation und erhalten

$$
S \sim \sum_{n=0}^{\infty} B_{n}(q) \tau^{n}
$$

mit

$$
B_{n}(q):=\frac{1}{2 \sqrt{\pi}} e^{i \pi / 8-i q^{2} / 2} \int_{\nearrow^{i q}} \frac{e^{i v^{2} / 2+q v}}{\cosh \frac{\sqrt{\pi}}{2} v} P_{n}(v-i q) d v \quad(n \geq 0) .
$$

Da wir die Reihe $\sum_{n=0}^{\infty} P_{n}(v-i q) \tau^{n}$, die nur für $|v-i q|<1 /(2 \tau)$ konvergiert, gliedweise über den unendlichen Integrationsweg $\gamma^{i q}$ integriert haben, können wir in 1.24 kein Gleichheitszeichen setzen. Das dort gewählte Zeichen „ “ besagt zunächst nur, daß die formale Potenzreihe $\sum_{n=0}^{\infty} B_{n}(q) \tau^{n}$ dem Ausdruck $S$ auf natürliche Weise zugeordnet ist. Es ist aber zu vermuten, daß es sich bei (1.24) um 
eine asymptotische Entwicklung des Ausdruckes $S$ für $\tau \rightarrow 0$ handelt. In Kapitel 3 werden wir das beweisen.

Wir tragen nun die Darstellung 1.22 der Polynome $P_{n}(z)$ in 1.25 ein. Es folgt

$$
B_{n}(q)=\sum_{k=0}^{n} i^{k-n} \frac{a_{k}^{(n)}}{(n+2 k) !} b_{n+2 k}(q) \quad(n \geq 0)
$$

mit

$$
b_{m}(q):=\frac{1}{2 \sqrt{\pi}} e^{i \pi / 8-i q^{2} / 2} \int_{\nearrow^{i q}} \frac{e^{i v^{2} / 2+q v}}{\cosh \frac{\sqrt{\pi}}{2} v}(v-i q)^{m} d v \quad(m \geq 0),
$$

so daß wir zur Berechnung der Koeffizienten $B_{n}(q)$ nur noch die Funktionen $b_{m}(q)$ zu bestimmen brauchen. Für $m=0$ folgt aus 1.13 die Beziehung $b_{0}(q)=\widetilde{F}(q)$. Für $m>0$ läßt sich $b_{m}(q)$ in endlicher Form mit Ableitungen der Funktion $\widetilde{F}(q)$ darstellen. Dazu ersetzen wir den Integrationsweg $\nearrow^{i q}$ in 1.13 mit $i \sqrt{\pi} \nearrow-i \sqrt{\pi}$ und schreiben $q+x$ für $q$. Wir erhalten

$$
\widetilde{F}(q+x)=\frac{1}{2 \sqrt{\pi}} e^{i \pi / 8-i\left(q^{2}+2 q x+x^{2}\right) / 2} \underset{i \sqrt{\pi} \nearrow-i \sqrt{\pi}}{\int \frac{e^{i v^{2} / 2+q v+x v}}{\cosh \frac{\sqrt{\pi}}{2} v}} d v
$$

und daraus

$$
\begin{aligned}
e^{i x^{2} / 2} \widetilde{F}(q+x) & =\frac{1}{2 \sqrt{\pi}} e^{i \pi / 8-i q^{2} / 2} \int \underset{i \sqrt{\pi} \nearrow-i \sqrt{\pi}}{\cosh \frac{\sqrt{\pi}}{2} v} e^{x(v-i q)} d v \\
& =\sum_{m=0}^{\infty}\left[\frac{1}{2 \sqrt{\pi}} e^{i \pi / 8-i q^{2} / 2} \int \frac{e^{i v^{2} / 2+q v}}{\cosh \frac{\sqrt{\pi}}{2} v}(v-i q)^{m} d v\right] \frac{x^{m}}{m !} .
\end{aligned}
$$

$\mathrm{Da}-\sqrt{\pi}<q \leq \sqrt{\pi}$ ist, können wir hier als Integrationsweg wieder $\nearrow^{i q}$ wählen. Ersetzen von $\widetilde{F}(q+x)$ mit der Taylorreihe $\sum_{m=0}^{\infty} \widetilde{F}^{(m)}(q) x^{m} / m$ ! und Vergleich mit (1.27) ergibt so die Formel

$$
e^{i x^{2} / 2} \sum_{m=0}^{\infty} \frac{\widetilde{F}^{(m)}(q)}{m !} x^{m}=\sum_{m=0}^{\infty} \frac{b_{m}(q)}{m !} x^{m},
$$

aus der sich die $b_{m}(q)$ durch Koeffizientenvergleich berechnen lassen, wenn man die Funktion $e^{i x^{2} / 2}$ mit ihrer Potenzreihe um den Punkt $x=0$ ersetzt und diese mit der Reihe $\sum_{m=0}^{\infty} \widetilde{F}^{(m)}(q) x^{m} / m$ ! multipliziert.

Wir bilden jetzt das Produkt der beiden formalen Potenzreihen 1.11 und (1.24. Die Koeffizienten in dem Ansatz

$$
U \cdot S \sim \sum_{n=0}^{\infty} \widetilde{C}_{n}(q) \tau^{n}
$$


berechnen sich dann nach den Formeln

$$
\begin{gathered}
\widetilde{C}_{2 n}(q)=\sum_{k=0}^{n} i^{k} \alpha_{k} B_{2 n-2 k}(q), \\
\widetilde{C}_{2 n+1}(q)=\sum_{k=0}^{n} i^{k} \alpha_{k} B_{2 n+1-2 k}(q) .
\end{gathered}
$$

Setzen wir noch

$$
\widehat{C}_{n}(q):=\Re \widetilde{C}_{n}(q) \quad(n \geq 0),
$$

so wird

$$
\Re(U \cdot S) \sim \sum_{n=0}^{\infty} \widehat{C}_{n}(q) \tau^{n},
$$

und aus Satz 1.2.1 folgt mit $(t /(2 \pi))^{1 / 4}=\sqrt{a}$ die formale Entwicklung für $Z(t)$

Satz 1.3.1 (Riemann-Siegel-Formel).

$$
Z(t) \sim 2 \sum_{n=1}^{N} \frac{\cos (\vartheta(t)-t \log n)}{\sqrt{n}}+\frac{(-1)^{N-1}}{\sqrt{a}} \sum_{n=0}^{\infty} \widehat{C}_{n}(q) \tau^{n}
$$

mit

$$
a:=\sqrt{\frac{t}{2 \pi}}, \quad N:=\lfloor a\rfloor, \quad q:=\sqrt{\pi}[1-2(a-N)], \quad \tau:=\frac{1}{2 \sqrt{2 t}} .
$$

In Kapitel 3 werden wir zeigen, daß es sich hierbei um eine asymptotische Entwicklung der Funktion $Z(t)$ für $t \rightarrow+\infty$ handelt. Die Identität unserer Darstellung mit der Riemann-Siegel-Formel folgt dann aus dem Eindeutigkeitssatz asymptotischer Entwicklungen durch Vergleich mit der von Siegel in [32. S. 290] angegebenen asymptotischen Entwicklung von $Z(t)$, wenn man diese auf unsere Form transformiert, d. h. mit den Größen $\tau$ und $q$ schreibt. Die Funktion $\widehat{F}(q)=\Im \widetilde{F}(q)$ wird daher in den Koeffizienten $\widehat{C}_{n}(q)$ nicht auftreten. Für $n=0$ kann man das direkt nachprüfen, denn in Übereinstimmung mit 1.16 wird $\widehat{C}_{0}(q)=\widehat{F}(q)$. Den allgemeinen Beweis findet man im nächsten Kapitel.

Nach den obenstehenden Formeln können die Koeffizienten $\widehat{C}_{n}(q)$ auf folgende Weise berechnet werden:

Man bestimme die $a_{k}^{(n)}$ aus 1.23 , die $b_{m}(q)$ aus 1.28 und damit die $B_{n}(q)$ aus 1.26. Mit den Zahlen $\alpha_{n}$ - numerische Werte findet man in Satz 4.2.4, S. 74 für $n \leq 8$ - erhält man dann die $\widetilde{C}_{n}(q)$ aus $[1.30$ ] und daraus die gesuchten $\widehat{C}_{n}(q)$ durch Übergang zum Realteil aus (1.31).

Der dafür notwendige Rechenaufwand ist jedoch untragbar. Im nächsten Kapitel werden wir ein Verfahren kennenlernen, das es gestattet, die $\widehat{C}_{n}(q)$ ohne Kenntnis $\operatorname{der} a_{k}^{(n)}, b_{m}(q), B_{n}(q)$ und $\alpha_{n}$ direkt zu berechnen. 


\section{Kapitel 2}

\section{Die Koeffizienten der Riemann-Siegel-Formel}

\subsection{Formeln zur Koeffizientenberechnung}

Die Koeffizienten $\widehat{C}_{n}(q)$ in der Riemann-Siegel-Formel aus Satz 1.3.1. S. 18 lassen sich auf folgende Weise sehr einfach berechnen:

Satz 2.1.1. Mit der Funktion

$$
\widehat{F}(q):=\frac{\cos \left(\frac{q^{2}}{2}+\frac{3 \pi}{8}\right)}{\cos \sqrt{\pi} q}
$$

wird

$$
\widehat{C}_{n}(q)=\sum_{k=0}^{\lfloor 3 n / 4\rfloor} d_{k}^{(n)} \frac{\widehat{F}^{(3 n-4 k)}(q)}{(3 n-4 k) !} \quad(n \geq 0) .
$$

Die $d_{k}^{(n)}$ sind positive rationale Zahlen, die der Rekursionsformel

$d_{k}^{(n+1)}=(3 n+1-4 k)(3 n+2-4 k) d_{k}^{(n)}+d_{k-1}^{(n)} \quad\left(n \geq 0,0 \leq k<\frac{3(n+1)}{4}\right)$,

$d_{k}^{(n)}=0 \quad$ für $k<0$ oder $k>\frac{3 n}{4}$,

$d_{3 n}^{(4 n)}=\lambda_{n} \quad(n \geq 0)$

genügen. Die Zahlen $\lambda_{n}$ sind rekursiv durch

$$
\begin{aligned}
\lambda_{0} & =1 \\
(n+1) \lambda_{n+1} & =\sum_{k=0}^{n} 2^{4 k+1}\left|E_{2 k+2}\right| \lambda_{n-k} \quad(n \geq 0)
\end{aligned}
$$

gegeben. Dabei sind die $E_{2 k}$ die Eulerschen Zahlen. Für die ersten $\lambda_{n}$ ergeben sich die Werte

$$
\lambda_{0}=1
$$




$$
\begin{array}{ll}
\lambda_{1}=2, & \lambda_{4}=2 \cdot 3 \cdot 7 \cdot 68111 \\
\lambda_{2}=2 \cdot 41, & \lambda_{5}=2^{2} \cdot 3 \cdot 47 \cdot 499 \cdot 4729 \\
\lambda_{3}=2^{2} \cdot 3 \cdot 881, & \lambda_{6}=2^{2} \cdot 3 \cdot 409 \cdot 193077047 .
\end{array}
$$

Alle hier auftretenden Zahlen sind prim.

Die $\widehat{C}_{n}(q)$ sind daher positive rationale Kombinationen gewisser Ableitungen von $\widehat{F}(q)$, nämlich von $\widehat{F}^{(3 n)}(q), \widehat{F}^{(3 n-4)}(q), \widehat{F}^{(3 n-8)}(q), \ldots, \widehat{F}^{\left(3 n-4 k^{\prime}\right)}(q)$, wo $k^{\prime}$ die größte ganze Zahl $\leq 3 n / 4$ ist.

Die $d_{k}^{(n)}$ lassen sich für $n<4 m$ sehr einfach aus 2.2 berechnen, wenn die Zahlen $\lambda_{n}=d_{3 n}^{(4 n)}$ für $0 \leq n<m$ bekannt sind, die man sehr rasch aus 2.4 bestimmen kann. Für $n \leq 6$ ergeben sich so die oben angegebenen numerischen Werte.

Da die $\lambda_{n}$ nach 2.4 positive rationale Zahlen sind, sind auch alle $d_{k}^{(n)}$ positiv rational; jedoch ist es sehr wahrscheinlich, daß alle $\lambda_{n}$ und damit auch alle $d_{k}^{(n)}$ natürliche Zahlen sind. Ein allgemeingültiger Beweis für diese Vermutung ist dem Autor nicht bekannt ${ }^{1)}$ Uns genügt die Tatsache, daß die $\lambda_{n}$ für $n \leq 6$ und demnach die $d_{k}^{(n)}$ für $n \leq 27$ natürliche Zahlen sind. In Tabelle II, S. 95 sind die $d_{k}^{(n)}-$ aus Gründen der Übersichtlichkeit in Primfaktoren zerlegt - für $n \leq 12$ angegeben.

Der Beweis von Satz 2.1.1 ist leider etwas aufwendig und wird den größten Teil dieses Abschnittes einnehmen. Wir treffen dazu folgende Konvention:

Unter dem Ausdruck „k.T.v. $(f(x))$ “ mit der Abkürzung „k.T.v.“ für „konstanter Term von" wollen wir das konstante Glied der - u. U. nur formalen - Laurententwicklung von $f(x)$ um den Punkt $x=0$ verstehen. Das soll auch dann gelten, wenn $f(x)$ außer von $x$ noch von anderen Variablen abhängt.

Mit Hilfe des nächsten Satzes können die Koeffizienten $B_{n}(q)$ der formalen Entwicklung $S \sim \sum_{n=0}^{\infty} B_{n}(q) \tau^{n}$ (vgl. in Abschnitt 1.3. S. 16 ff.) ohne Kenntnis der Zahlen $a_{k}^{(n)}$ und der Funktionen $b_{m}(q)$ direkt berechnet werden:

Satz 2.1.2. Für $n \geq 0$ ist

$$
B_{n}(q)=\mathrm{k} . \mathrm{T} . \mathrm{v} \cdot\left(A_{n}(x) \sum_{m=0}^{\infty} \frac{\widetilde{F}^{(m)}(q)}{m !} x^{m}\right)
$$

Die Funktionen $A_{n}(x)$ sind rekursiv durch

$$
\begin{aligned}
A_{0}(x) & =e^{i x^{2} / 2} \\
A_{n+1}(x) & =x A_{n}(x)+\left(\frac{A_{n}(x)}{x}\right)^{\prime \prime} \quad(n \geq 0)
\end{aligned}
$$

gegeben.

\footnotetext{
1) Arias de Reyna 2] hat diese Vermutung 2003 bewiesen.
} 
Beweis. Wir führen folgende Polynome vom Grade $3 n$ in $x^{-1}$ ein

$$
Q_{n}(x):=\sum_{k=0}^{n} i^{k-n} a_{k}^{(n)} x^{-n-2 k} \quad(n \geq 0)
$$

und setzen damit

$$
A_{n}(x):=e^{i x^{2} / 2} Q_{n}(x) \quad(n \geq 0) .
$$

Die $a_{k}^{(n)}$ sind die in 1.23 , S. 16 rekursiv eingeführten natürlichen Zahlen.

Wir multiplizieren Gleichung 1.28, S. 17 mit $Q_{n}(x)$ und erhalten wegen 2.8

$$
A_{n}(x) \sum_{m=0}^{\infty} \frac{\widetilde{F}^{(m)}(q)}{m !} x^{m}=Q_{n}(x) \sum_{m=0}^{\infty} \frac{b_{m}(q)}{m !} x^{m} \quad(n \geq 0) .
$$

Da $Q_{n}(x)$ als Polynom in $x^{-1}$ den Grad $3 n$ hat, lassen sich hier beide Seiten der Gleichung in beständig konvergierende Laurentreihen mit endlichem Hauptteil um den Punkt $x=0$ entwickeln und die höchste nicht verschwindende negative Potenz von $x$ ist $x^{-3 n}$. Wir vergleichen die konstanten Glieder dieser beiden Laurentreihen:

$$
\text { k.T.v. }\left(Q_{n}(x) \sum_{m=0}^{\infty} \frac{b_{m}(q)}{m !} x^{m}\right)=\sum_{k=0}^{n} i^{k-n} \frac{a_{k}^{(n)}}{(n+2 k) !} b_{n+2 k}(q)=B_{n}(q)
$$

unter Verwendung von 1.26, S. 17. Folglich ist auch

$$
B_{n}(q)=\text { k.T.v. }\left(A_{n}(x) \sum_{m=0}^{\infty} \frac{\widetilde{F}^{(m)}(q)}{m !} x^{m}\right) \quad(n \geq 0)
$$

und 2.5 ist bewiesen.

Zum Beweis von (2.6) leiten wir zunächst eine Rekursionsformel für die $Q_{n}(x)$ her. Aus den Eigenschaften der Zahlen $a_{k}^{(n)}-$ man beachte besonders $a_{k}^{(n)}=0$ für $k<0$ oder $k>n$ - folgen für $n \geq 0$ die Gleichungen

$$
\begin{aligned}
\frac{Q_{n}(x)}{x} & =\sum_{k=0}^{n} i^{k-n} a_{k}^{(n)} x^{-n-2 k-1} \\
& =\sum_{k=0}^{n+1} i^{k-n} a_{k}^{(n)} x^{-n-2 k-1} \\
\left(\frac{Q_{n}(x)}{x}\right)^{\prime} & =-\sum_{k=0}^{n} i^{k-n}(n+2 k+1) a_{k}^{(n)} x^{-n-2 k-2} \\
& =-\sum_{k=0}^{n+1} i^{k-n}(n+2 k+1) a_{k}^{(n)} x^{-n-2 k-2} \\
\left(\frac{Q_{n}(x)}{x}\right)^{\prime \prime} & =\sum_{k=0}^{n} i^{k-n}(n+2 k+1)(n+2 k+2) a_{k}^{(n)} x^{-n-2 k-3} \\
& =\sum_{k=0}^{n+1} i^{k-n-1}(n+2 k-1)(n+2 k) a_{k-1}^{(n)} x^{-n-2 k-1} .
\end{aligned}
$$


(Die hochgestellten Striche bedeuten natürlich ein- bzw. zweimalige Differentiation nach $x$ ). Damit wird unter Beachtung von 1.23

$$
\begin{aligned}
& \left(\frac{Q_{n}(x)}{x}\right)^{\prime \prime}+2 i x\left(\frac{Q_{n}(x)}{x}\right)^{\prime}+i \frac{Q_{n}(x)}{x} \\
= & \sum_{k=0}^{n+1} i^{k-n-1}(n+2 k-1)(n+2 k) a_{k-1}^{(n)} x^{-n-2 k-1} \\
& -\sum_{k=0}^{n+1} i^{k-n+1}(2 n+4 k+2) a_{k}^{(n)} x^{-n-2 k-1} \\
& +\sum_{k=0}^{n+1} i^{k-n+1} a_{k}^{(n)} x^{-n-2 k-1} \\
= & \sum_{k=0}^{n+1} i^{k-n-1}\left[(n+2 k-1)(n+2 k) a_{k-1}^{(n)}+(2 n+4 k+1) a_{k}^{(n)}\right] x^{-n-2 k-1} \\
= & \sum_{k=0}^{n+1} i^{k-n-1} a_{k}^{(n+1)} x^{-n-2 k-1}=Q_{n+1}(x),
\end{aligned}
$$

und wir haben die Rekursionsformel

$$
\begin{gathered}
Q_{0}(x) \equiv 1 \\
Q_{n+1}(x)=\left(\frac{Q_{n}(x)}{x}\right)^{\prime \prime}+2 i x\left(\frac{Q_{n}(x)}{x}\right)^{\prime}+i \frac{Q_{n}(x)}{x} \quad(n \geq 0)
\end{gathered}
$$

gewonnen. Die Anfangsbedingung folgt wegen $a_{0}^{(0)}=1$ direkt aus 2.7). Es ist aber nach 2.8

$$
Q_{n}(x)=e^{-i x^{2} / 2} A_{n}(x) \quad(n \geq 0)
$$

und daher, wie eine einfache Rechnung zeigt,

$$
\begin{aligned}
\frac{Q_{n}(x)}{x} & =e^{-i x^{2} / 2} \frac{A_{n}(x)}{x} \\
\left(\frac{Q_{n}(x)}{x}\right)^{\prime} & =e^{-i x^{2} / 2}\left[\left(\frac{A_{n}(x)}{x}\right)^{\prime}-i x\left(\frac{A_{n}(x)}{x}\right)\right], \\
\left(\frac{Q_{n}(x)}{x}\right)^{\prime \prime} & =e^{-i x^{2} / 2}\left[\left(\frac{A_{n}(x)}{x}\right)^{\prime \prime}-2 i x\left(\frac{A_{n}(x)}{x}\right)^{\prime}-\left(x^{2}+i\right) \frac{A_{n}(x)}{x}\right] .
\end{aligned}
$$

Wir setzen das in $(2.9)$ ein und erhalten nach Kürzen von $e^{-i x^{2} / 2}$ für $n \geq 0$

$$
A_{n+1}(x)=\left(\frac{A_{n}(x)}{x}\right)^{\prime \prime}-2 i x\left(\frac{A_{n}(x)}{x}\right)^{\prime}-\left(x^{2}+i\right) \frac{A_{n}(x)}{x}
$$




$$
\begin{aligned}
& +2 i x\left(\frac{A_{n}(x)}{x}\right)^{\prime}+2 x^{2} \frac{A_{n}(x)}{x}+i \frac{A_{n}(x)}{x} \\
= & x A_{n}(x)+\left(\frac{A_{n}(x)}{x}\right)^{\prime \prime},
\end{aligned}
$$

also gerade die Behauptung 2.6. Da $Q_{0}(x) \equiv 1$ ist, folgt $A_{0}(x)=e^{i x^{2} / 2}$ unmittelbar aus 2.8 und der Satz ist bewiesen.

Aus 2.6 kann man die allgemeine Form der Laurententwicklungen von $A_{n}(x)$ und damit aus 2.5 die allgemeine Form der Koeffizienten $B_{n}(q)$ bestimmen:

Corollar 2.1.1. Für geraden Index besitzen die $A_{n}(x)$ eine gerade und für ungeraden Index eine ungerade Laurententwicklung um den Punkt $x=0$. Die höchste nicht verschwindende negative $x$-Potenz ist $x^{-3 n}$. Die Koeffizienten $B_{n}(q)$ sind daher für geraden Index eine Kombination von geraden und für ungeraden Index eine Kombination von ungeraden Ableitungen der Funktion $\widetilde{F}(q)$. Die höchste in $B_{n}(q)$ auftretende Ableitung ist $\widetilde{F}^{(3 n)}(q)$.

Beginnend mit der Potenzreihe $A_{0}(x)=e^{i x^{2} / 2}=\sum_{m=0}^{\infty}(i / 2)^{m} x^{2 m} / m$ ! ergeben sich die Laurententwicklungen der $A_{n}(x)$ rekursiv aus $[2.6]$ und damit die $B_{n}(q)$ aus 2.5. Da diese für uns aber nur von geringem Interesse sind, verzichten wir darauf und wenden uns gleich der Berechnung der Koeffizienten $\widetilde{C}_{n}(q)$ in der formalen Reihe $U \cdot S \sim \sum_{n=0}^{\infty} \widetilde{C}_{n}(q) \tau^{n}$ von Gleichung 1.29 , S. $17 \mathrm{zu}$.

Satz 2.1.3. Mit den $A_{n}(x)$ aus Satz 2.1.2 und den Koeffizienten $\alpha_{n}$ der formalen Potenzreihe $U \sim \sum_{n=0}^{\infty} i^{n} \alpha_{n} \tau^{2 n}$ aus Satz 4.2.4. S. 74 setzen wir

$$
\begin{aligned}
D_{2 n}(x) & :=\sum_{k=0}^{n} i^{k} \alpha_{k} A_{2 n-2 k}(x), \\
D_{2 n+1}(x) & :=\sum_{k=0}^{n} i^{k} \alpha_{k} A_{2 n+1-2 k}(x) .
\end{aligned}
$$

Dann ist

$$
\widetilde{C}_{n}(q)=\text { k.T.v. }\left(D_{n}(x) \sum_{m=0}^{\infty} \frac{\widetilde{F}^{(m)}(q)}{m !} x^{m}\right) \quad(n \geq 0)
$$

und die Funktionen $D_{n}(x)$ genügen der Rekursionsformel

$$
\begin{gathered}
D_{0}(x)=e^{i x^{2} / 2}, \\
D_{2 n+1}(x)=x D_{2 n}(x)+\left(\frac{D_{2 n}(x)}{x}\right)^{\prime \prime}, \quad(n \geq 0) \\
D_{2 n+2}(x)=x D_{2 n+1}(x)+\left(\frac{D_{2 n+1}(x)}{x}\right)^{\prime \prime}+i^{n+1} \alpha_{n+1} e^{i x^{2} / 2} .
\end{gathered}
$$


Der Beweis ist trivial. Nach Definition der Funktionen $D_{n}(x)$ in 2.10 ergibt sich nämlich 2.11 unter Beachtung der Gleichungen 1.30 aus 2.5 und die Rekursionsformel (2.12) unmittelbar aus (2.6).

Mit Hilfe dieses Satzes kann man die Koeffizienten $\widetilde{C}_{n}(q)$ bereits direkt berechnen. Da aber in die Auswertung der Formel 2.11 nur der Hauptteil und das konstante Glied der Laurententwicklung von $D_{n}(x)$ um den Punkt $x=0$ eingeht, während die rekursive Berechnung dieser Hauptteile und konstanten Glieder nach 2.12 neben den Zahlen $\alpha_{n}$ noch die Kenntnis gewisser positiver $x$-Potenzen verlangt, wünscht man sich zur Verminderung des Rechenaufwandes eine Formel, die zur Bestimmung des Hauptteils und des konstanten Gliedes der Laurententwicklung von $D_{n+1}(x)$ weitgehend nur den Hauptteil und das konstante Glied der Laurententwicklung von $D_{n}(x)$ benötigt. Eine solche Formel läßt sich mit Hilfe einiger zusätzlicher Informationen über die allgemeine Form dieser Haupteile und konstanten Glieder gewinnen. Als erste Informationsquelle ist hier das Corollar 2.1.1 zu erwähnen, das auch mit $D_{n}(x)$ statt $A_{n}(x)$ und mit $\widetilde{C}_{n}(q)$ statt $B_{n}(q)$ richtig bleibt, wenn man die Gleichungen 2.10 und 2.11) berücksichtigt. Die genaue Form der Hauptteile und konstanten Glieder der Funktionen $D_{n}(x)$ ergibt sich aus dem folgenden Satz.

Satz 2.1.4. Die formale Reihe

$$
U \cdot S \sim \sum_{n=0}^{\infty} \widetilde{C}_{n}(q) \tau^{n}
$$

läßt sich in eine nach den Ableitungen von $\widetilde{F}(q)$ angeordnete Reihe umordnen:

$$
\sum_{n=0}^{\infty} \widetilde{C}_{n}(q) \tau^{n} \doteq \sum_{n=0}^{\infty} \mathcal{D}_{n}(\tau) \frac{\widetilde{F}^{(n)}(q)}{n !}
$$

Die Funktionen $\mathcal{D}_{n}(\tau)$ sind formale Potenzreihen in $\tau$. Sie genügen der Rekursionsformel

$$
\begin{array}{cc}
\mathcal{D}_{n+1}(\tau) \doteq \frac{\mathcal{D}_{n}(\tau)}{\tau} & (n=1,2), \\
\mathcal{D}_{n+1}(\tau) \doteq \frac{\mathcal{D}_{n}(\tau)}{\tau}-(n-1)(n-2) \mathcal{D}_{n-3}(\tau) & (n \geq 3)
\end{array}
$$

mit den Anfangsbedingungen

$$
\begin{aligned}
& \mathcal{D}_{0}(\tau) \doteq \sum_{n=0}^{\infty} \lambda_{n} \tau^{4 n} \\
& \mathcal{D}_{1}(\tau) \doteq \sum_{n=1}^{\infty} \mu_{n} \tau^{4 n-1} .
\end{aligned}
$$

Die Koeffizienten $\lambda_{n}$ sind rekursiv durch

$$
\begin{aligned}
\lambda_{0} & =1, \\
(n+1) \lambda_{n+1} & =\sum_{k=0}^{n} 2^{4 k+1}\left|E_{2 k+2}\right| \lambda_{n-k} \quad(n \geq 0)
\end{aligned}
$$


gegeben. Die Koeffizienten $\mu_{n}$ bestimmen sich mit den durch

$$
\begin{aligned}
\varrho_{0} & =-1 \\
(n+1) \varrho_{n+1} & =-\sum_{k=0}^{n} 2^{4 k+1}\left|E_{2 k+2}\right| \varrho_{n-k} \quad(n \geq 0)
\end{aligned}
$$

rekursiv definierten Zahlen $\varrho_{n}$ aus

$$
\mu_{n}=\frac{\lambda_{n}+\varrho_{n}}{2} \quad(n \geq 1)
$$

Die $E_{2 k}$ sind die Eulerschen Zahlen.

Beweis. Wir betrachten die formale Potenzreihe

$$
G(\tau, x): \doteq \sum_{n=0}^{\infty} D_{n}(x) \tau^{n}
$$

wobei wir unter $D_{n}(x)$ von jetzt ab immer die Laurententwicklung dieser Funktion um den Punkt $x=0$ verstehen wollen. Damit folgt aus 2.11

$$
\text { k.T.v. }\left(G(\tau, x) \sum_{m=0}^{\infty} \frac{\widetilde{F}^{(m)}(q)}{m !} x^{m}\right) \doteq \sum_{n=0}^{\infty} \widetilde{C}_{n}(q) \tau^{n} \sim U \cdot S .
$$

Nach den Rechengesetzen für formale Potenzreihen ist es möglich, die Reihe in (2.19) in eine nach Potenzen von $x$ angeordnete Reihe umzuordnen. Wir erhalten auf diese Weise die formale Laurentreihe mit unendlichem Hauptteil

$$
G(\tau, x) \doteq \sum_{n=-\infty}^{+\infty} \mathcal{D}_{-n}(\tau) x^{n}
$$

in der die Koeffizienten $\mathcal{D}_{n}(\tau)$ formale Potenzreihen in $\tau$ sind. Trägt man diese Darstellung von $G(\tau, x)$ in 2.20 ein, so folgt wegen

$$
\text { k.T.v. }\left(G(\tau, x) \sum_{m=0}^{\infty} \frac{\widetilde{F}^{(m)}(q)}{m !} x^{m}\right) \doteq \sum_{n=0}^{\infty} \mathcal{D}_{n}(\tau) \frac{\widetilde{F}^{(n)}(q)}{n !}
$$

die im Sinne formaler Potenzreihen zu verstehende Gleichung

$$
\sum_{n=0}^{\infty} \widetilde{C}_{n}(q) \tau^{n} \doteq \sum_{n=0}^{\infty} \mathcal{D}_{n}(\tau) \frac{\widetilde{F}^{(n)}(q)}{n !}
$$

die besagt, daß eine jede dieser beiden Reihen durch Umordnung aus der anderen hervorgeht. Damit ist 2.13) bewiesen.

Zur Herleitung der Rekursionsformel 2.14 betrachten wir den Ausdruck

$$
G(\tau, x)-\tau\left[x G(\tau, x)+\left(\frac{G(\tau, x)}{x}\right)^{\prime \prime}\right],
$$


in dem die hochgestellten Striche zweimalige Differentiation nach $x$ bedeuten. Mit der Reihe 2.19) und den Formeln 2.12 wird dieser Ausdruck gleich

$e^{i x^{2} / 2}+\sum_{n=0}^{\infty} D_{n+1}(x) \tau^{n+1}-\sum_{n=0}^{\infty}\left[x D_{n}(x)+\left(\frac{D_{n}(x)}{x}\right)^{\prime \prime}\right] \tau^{n+1} \doteq e^{i x^{2} / 2} \sum_{n=0}^{\infty} i^{n} \alpha_{n} \tau^{2 n}$,

so daß $G(\tau, x)$ der Differentialgleichung

$$
G(\tau, x)-\tau\left[x G(\tau, x)+\left(\frac{G(\tau, x)}{x}\right)^{\prime \prime}\right] \doteq e^{i x^{2} / 2} \sum_{n=0}^{\infty} i^{n} \alpha_{n} \tau^{2 n}
$$

genügt. Setzen wir hier für $G(\tau, x)$ die Laurentreihe 2.21 ein, so erhalten wir für die linke Seite der Differentialgleichung die Laurentreihe

$$
\begin{gathered}
\sum_{n=-\infty}^{+\infty} \mathcal{D}_{-n}(\tau) x^{n}-\sum_{n=-\infty}^{+\infty} \tau \mathcal{D}_{-n}(\tau) x^{n+1}-\sum_{n=-\infty}^{+\infty} \tau \mathcal{D}_{-n}(\tau)(n-1)(n-2) x^{n-3} \\
\doteq \sum_{n=-\infty}^{+\infty}\left[\mathcal{D}_{-n}(\tau)-\tau \mathcal{D}_{-n+1}(\tau)-(n+2)(n+1) \tau \mathcal{D}_{-n-3}(\tau)\right] x^{n}
\end{gathered}
$$

Da die rechte Seite der Differentialgleichung bei $x=0$ regulär ist, muß der Hauptteil dieser Laurentreihe verschwinden. Wir haben also

$$
\mathcal{D}_{-n}(\tau)-\tau \mathcal{D}_{-n+1}(\tau)-(n+2)(n+1) \tau \mathcal{D}_{-n-3}(\tau) \doteq 0
$$

für $n=-1,-2,-3, \ldots$, und das ist gerade (2.14), wenn man $n$ mit $-n$ ersetzt.

Für $n \geq 2$ lassen sich die formalen Potenzreihen $\mathcal{D}_{n}(\tau)$ aus (2.14) rekursiv berechnen, falls $\mathcal{D}_{0}(\tau)$ und $\mathcal{D}_{1}(\tau)$ bekannt sind. Auf dem im Beweis von Satz 4.3.1. S. 76 beschriebenen Wege gelingt es, diese zu bestimmen und so die Formeln (2.15) bis (2.18) zu beweisen. Für $n \leq 6$ findet man dort neben den numerischen Werten der $\lambda_{n}$, die wir schon in Satz 2.1.1 angegeben haben, auch numerische Werte für die Zahlen $\varrho_{n}$ und $\mu_{n}$. Der Beweis ist damit abgeschlossen.

Mit den Ergebnissen des letzten Satzes können wir jetzt Satz 2.1.1 beweisen. Zunächst folgt aus (2.14) und den Anfangsbedingungen (2.15), daß die Koeffizienten der formalen Potenzreihen $\mathcal{D}_{n}(\tau)$ für jedes $n \geq 0$ rationale Zahlen und darum insbesondere reell sind. Ferner müssen die Exponenten aller in $\mathcal{D}_{n}(\tau)$ vorkommenden Potenzen von $\tau \equiv-n \bmod 4$ sein. Nach 2.13 sind die Koeffizienten $\widetilde{C}_{n}(q)$ daher eine reelle und rationale Kombination von Ableitungen der Funktion $\widetilde{F}(q)$ und die Ordnung einer jeden in $\widetilde{C}_{n}(q)$ auftretenden Ableitung von $\widetilde{F}(q)$ ist $\equiv-n$ mod 4. Da nach Corollar 2.1.1 - mit $\widetilde{C}_{n}(q)$ statt $B_{n}(q)$ - die höchste in $\widetilde{C}_{n}(q)$ vorkommende Ableitung $\widetilde{F}^{(3 n)}(q)$ ist und die Kongruenz $3 n \equiv-n \bmod 4$ gilt, haben die $\widetilde{C}_{n}(q)$ die allgemeine Form

$$
\widetilde{C}_{n}(q)=\sum_{0 \leq k \leq \frac{3 n}{4}} d_{k}^{(n)} \frac{\widetilde{F}^{(3 n-4 k)}(q)}{(3 n-4 k) !} \quad(n \geq 0)
$$

2) Die in 2.21 auftretenden $\mathcal{D}_{n}(\tau)$ mit negativem Index sind für uns uninteressant, da sie in der Gleichung 2.13, nicht vorkommen. 
mit gewissen rationalen Zahlen $d_{k}^{(n)}$. Durch Übergang zum Realteil ergibt sich hieraus die in Satz 2.1.1 angegebene Darstellung der Koeffizienten; denn nach 1.15, S. 13 und (1.31), S. 18 braucht man dazu nur das Zeichen ", mit dem Zeichen ," $" \mathrm{zu}$ ersetzen.

Nach 2.15 ist $\lambda_{n}$ der Koeffizient von $\tau^{4 n}$ in $\mathcal{D}_{0}(\tau)$. Mit 2.13 folgt daraus, daß die Funktion $F(q)$ für jedes $n \geq 0$ in $\widetilde{C}_{4 n}(q)$ genau $\lambda_{n}$-mal auftritt. Andererseits ist - wie aus 2.22 ersichtlich - der Koeffizient von $\widetilde{F}(q)$ in $\widetilde{C}_{4 n}(q)$ gerade $d_{3 n}^{(4 n)}$, so daß für alle $n \geq 0$ die Beziehung $d_{3 n}^{(4 n)}=\lambda_{n}$ besteht. Zusammen mit (2.16) sind damit (2.3) und (2.4) bewiesen.

Zum Beweis der Rekursionsformel (2.2) vergleichen wir 2.22$)$ und (2.11). Wir erhalten:

$$
\begin{aligned}
& \text { Hauptteil und konstantes Glied von } D_{n}(x) \\
& \text { hat für } n \geq 0 \text { die Form } \sum_{0 \leq k \leq \frac{3 n}{4}} d_{k}^{(n)} x^{-3 n+4 k} \text {. }
\end{aligned}
$$

Schreiben wir zur Abkürzung „H.T.v.“ für „Hauptteil von“ und nehmen in den Formeln (2.12 auf beiden Seiten den Hauptteil, so lassen sich diese zu

$$
\text { H.T.v. }\left[D_{n+1}(x)\right]=\text { H.T.v. }\left[x D_{n}(x)+\left(\frac{D_{n}(x)}{x}\right)^{\prime \prime}\right] \quad(n \geq 0)
$$

zusammenfassen. Man sieht unmittelbar, daß die positiven Potenzen von $D_{n}(x)$ zur Auswertung der rechten Seite dieser Gleichung nicht benötigt werden, so daß von $D_{n}(x)$ nur der Hauptteil und das konstante Glied bekannt zu sein brauchen. Wir setzen zur Vereinfachung noch $d_{k}^{(n)}:=0$ für $k<0$ oder $k>3 n / 4$ und erhalten mit 2.23) einerseits

$$
\begin{gathered}
\text { H.T.v. }\left[\sum_{0 \leq k \leq \frac{3 n}{4}} d_{k}^{(n)} x^{-3 n+4 k+1}+\sum_{0 \leq k \leq \frac{3 n}{4}}(3 n-4 k+1)(3 n-4 k+2) d_{k}^{(n)} x^{-3 n+4 k-3}\right] \\
=\text { H.T.v. }\left[\sum_{0 \leq k \leq \frac{3 n}{4}+1} d_{k-1}^{(n)} x^{-3 n+4 k-3}\right] \\
+\sum_{0 \leq k<\frac{3(n+1)}{4}}(3 n+1-4 k)(3 n+2-4 k) d_{k}^{(n)} x^{-3 n+4 k-3} \\
=\sum_{0 \leq k<\frac{3(n+1)}{4}}\left[(3 n+1-4 k)(3 n+2-4 k) d_{k}^{(n)}+d_{k-1}^{(n)}\right] x^{-3 n+4 k-3}
\end{gathered}
$$

und andererseits

$$
\text { H.T.v. }\left[D_{n+1}(x)\right]=\sum_{0 \leq k<\frac{3(n+1)}{4}} d_{k}^{(n+1)} x^{-3 n+4 k-3},
$$


woraus durch Koeffizientenvergleich die Rekursionsformel 2.2 folgt. Die Zahlen $d_{3 n}^{(4 n)}$ - das sind die konstanten Glieder von $D_{4 n}(x)$ werden von dieser Rekursionsformel nicht erfaßt. Da wir sie jedoch oben bereits auf anderem Wege bestimmt haben, ist das nicht wesentlich. Damit ist Satz 2.1.1 vollständig bewiesen.

Die Idee, die Reihe $\sum_{n=0}^{\infty} \widetilde{C}_{n}(q) \tau^{n}$ in eine nach Ableitungen von $\widetilde{F}(q)$ angeordnete Reihe umzuordnen, um so die genaue Form der Koeffizienten $\widetilde{C}_{n}(q) \mathrm{zu}$ bestimmen, stammt im wesentlichen von Riemann. Der Leser vergleiche hierzu Siegel [32. S. $290 \mathrm{ff}$. In etwas anderer Form findet man dort auch die Formeln 2.14 und 2.15]. Das in Satz 2.1.1 angegebene einfache Verfahren zur Berechnung der Koeffizienten der Riemann-Siegel-Formel ist aber bei Siegel und auch bei Edwards [16. Ch. 7] noch nicht vorhanden und daher neu 4$)$

Der jetzt folgende Satz ist für verschiedene Kontrollrechnungen nützlich.

Satz 2.1.5. Für $n \geq 0$ ist

$$
\begin{aligned}
\widehat{C}_{4 n}(\sqrt{\pi}) & =(-1)^{n} \alpha_{2 n} \cos \frac{\pi}{8}, \\
\widehat{C}_{4 n+2}(\sqrt{\pi}) & =(-1)^{n+1} \alpha_{2 n+1} \sin \frac{\pi}{8}
\end{aligned}
$$

und

$$
\begin{gathered}
\sum_{k=0}^{3 n}(-1)^{k} \frac{d_{k}^{(4 n)}}{2^{6 n-2 k}(6 n-2 k) !}=\alpha_{2 n}, \\
\sum_{k=0}^{3 n+1}(-1)^{k} \frac{d_{k}^{(4 n+2)}}{2^{6 n+3-2 k}(6 n+3-2 k) !}=\alpha_{2 n+1} .
\end{gathered}
$$

Beweis. Wir setzen in der Riemann-Siegel-Formel (Satz 1.3.1. S. 18 $t=t_{M}:=$ $2 \pi M^{2}$ mit ganzzahligem $M>0$. Dann wird $a=N=M, q=\sqrt{\pi}$ und $\tau=\tau_{M}=$ $1 /\left(2 \sqrt{2 t_{M}}\right)$. Nach Satz 3.2.1 des nächsten Kapitels auf Seite 53 stellt die RiemannSiegel-Formel die Funktion $Z(t)$ für $t \rightarrow+\infty$ bzw. $\tau \rightarrow 0$ asymptotisch dar. Da $\tau \rightarrow 0$ erfüllt ist, wenn $\tau$ speziell die Folge $\tau_{M}, M=1,2,3, \ldots$ durchläuft, ist daher

$$
Z\left(t_{M}\right) \sim 2 \sum_{n=1}^{M} \frac{\cos \left(\vartheta\left(t_{M}\right)-t_{M} \log n\right)}{\sqrt{n}}+\frac{(-1)^{M-1}}{\sqrt{M}} \sum_{n=0}^{\infty} \widehat{C}_{n}(\sqrt{\pi}) \tau_{M}^{n}
$$

für $\tau_{M} \rightarrow 0$.

Eine zweite Darstellung für $Z\left(t_{M}\right)$ ergibt sich, wenn wir in der Riemann-SiegelFormel den linksseitigen Grenzwert $t \rightarrow t_{M}$ mit $t<t_{M}$ bilden. Wegen $N=M-1$ und $q=-\sqrt{\pi}$ wird dann nämlich

$$
Z\left(t_{M}\right) \sim 2 \sum_{n=1}^{M-1} \frac{\cos \left(\vartheta\left(t_{M}\right)-t_{M} \log n\right)}{\sqrt{n}}-\frac{(-1)^{M-1}}{\sqrt{M}} \sum_{n=0}^{\infty} \widehat{C}_{n}(-\sqrt{\pi}) \tau_{M}^{n}
$$

\footnotetext{
3) Nach 2.23 besitzen nur diejenigen $D_{n}(x)$ ein konstantes Glied, deren Index durch 4 teilbar ist.

4) Der Leser vergleiche jedoch die Bemerkung am Ende dieses Abschnittes auf Seite 31 in Zusammenhang mit der untenstehenden Formel 2.27.
} 
für $\tau_{M} \rightarrow 0$. Subtrahieren wir diese Darstellung für $Z\left(t_{M}\right)$ von der ersten, so erhalten wir nach Kürzen von $2 / \sqrt{M}$, wenn wir noch beachten, daß die $\widehat{C}_{n}(q)$ mit geradem Index gerade und die mit ungeradem Index ungerade Funktionen von $q$ sind 5

$$
\cos \left(\vartheta\left(t_{M}\right)-t_{M} \log M\right) \sim(-1)^{M} \sum_{n=0}^{\infty} \widehat{C}_{2 n}(\sqrt{\pi}) \tau_{M}^{2 n} \quad\left(\tau_{M} \rightarrow 0\right) .
$$

Wegen

$$
t_{M} \log M=\frac{t_{M}}{2} \log \frac{t_{M}}{2 \pi}-\frac{t_{M}}{2}+\pi M^{2}
$$

ist daher

$$
\cos \left(\frac{t_{M}}{2} \log \frac{t_{M}}{2 \pi}-\frac{t_{M}}{2}-\vartheta\left(t_{M}\right)\right) \sim \sum_{n=0}^{\infty} \widehat{C}_{2 n}(\sqrt{\pi}) \tau_{M}^{2 n} \quad\left(\tau_{M} \rightarrow 0\right) .
$$

Ein Vergleich mit der aus Satz 4.2.4. S. 74 folgenden asymptotischen Entwicklung

$$
\begin{gathered}
\cos \left(\frac{t}{2} \log \frac{t}{2 \pi}-\frac{t}{2}-\vartheta(t)\right) \\
=\cos \frac{\pi}{8} \cos \left(\frac{t}{2} \log \frac{t}{2 \pi}-\frac{t}{2}-\frac{\pi}{8}-\vartheta(t)\right) \\
-\sin \frac{\pi}{8} \sin \left(\frac{t}{2} \log \frac{t}{2 \pi}-\frac{t}{2}-\frac{\pi}{8}-\vartheta(t)\right) \\
=\cos \frac{\pi}{8} \Re(U)-\sin \frac{\pi}{8} \Im(U) \\
\sim \cos \frac{\pi}{8} \sum_{n=0}^{\infty}(-1)^{n} \alpha_{2 n} \tau^{4 n}-\sin \frac{\pi}{8} \sum_{n=0}^{\infty}(-1)^{n} \alpha_{2 n+1} \tau^{4 n+2}
\end{gathered}
$$

ergibt dann in einfacher Weise die Gleichheit der beiden formalen Potenzreihen

$$
\cos \frac{\pi}{8} \sum_{n=0}^{\infty}(-1)^{n} \alpha_{2 n} \tau^{4 n}-\sin \frac{\pi}{8} \sum_{n=0}^{\infty}(-1)^{n} \alpha_{2 n+1} \tau^{4 n+2} \doteq \sum_{n=0}^{\infty} \widehat{C}_{2 n}(\sqrt{\pi}) \tau^{2 n}
$$

woraus wir die Formeln (2.24) durch Koeffizientenvergleich erhalten.

Mit den in Satz 4.1.5. S. 64 angegebenen Werten der geraden Ableitungen von $\widehat{F}(q)$ an der Stelle $\sqrt{\pi}$ ist

$$
\begin{aligned}
\frac{\widehat{F}^{(12 n-4 k)}(\sqrt{\pi})}{(12 n-4 k) !} & =\frac{(-1)^{n+k}}{2^{6 n-2 k}(6 n-2 k) !} \cos \frac{\pi}{8}, \\
\frac{\widehat{F}^{(12 n+6-4 k)}(\sqrt{\pi})}{(12 n+6-4 k) !} & =\frac{(-1)^{n+1+k}}{2^{6 n+3-2 k}(6 n+3-2 k) !} \sin \frac{\pi}{8} .
\end{aligned}
$$

Für $q=\sqrt{\pi}$ folgt damit aus 2.1

$$
\widehat{C}_{4 n}(\sqrt{\pi})=\cos \frac{\pi}{8} \sum_{k=0}^{3 n}(-1)^{n+k} \frac{d_{k}^{(4 n)}}{2^{6 n-2 k}(6 n-2 k) !},
$$

5) Das folgt aus 2.1, weil $\widehat{F}(q)$ eine gerade Funktion von $q$ ist.

6) In dem Ausdruck „, $(12 n-4 k)$ !“ fehlt in der Originalarbeit das „n“. 


$$
\widehat{C}_{4 n+2}(\sqrt{\pi})=\sin \frac{\pi}{8} \sum_{k=0}^{3 n+1}(-1)^{n+1+k} \frac{d_{k}^{(4 n+2)}}{2^{6 n+3-2 k}(6 n+3-2 k) !} .
$$

Hieraus erhalten wir 2.25) durch Vergleich mit 2.24).

Die Formeln 2.25 kann man für Kontrollrechnungen verwenden. Sämtliche $d_{k}^{(2 n)}$ aus Tabelle II wurden mit diesen Formeln überprüft. In allen Fällen ergab sich Übereinstimmung mit den Werten der $\alpha_{n}$ aus Satz 4.2.4. S. 74. Wir können daher davon ausgehen, daß die $d_{k}^{(n)}$ in Tabelle II korrekt wiedergegeben sind.7)

Wir transformieren jetzt unsere Darstellung der Riemann-Siegel-Formel auf die von Lehmer in 22] eingeführte Form, die zur numerischen Berechnung der Funktion $Z(t)$ heute gewöhnlich verwendet wird. Man findet die Riemann-Siegel-Formel in dieser Form auch in [15] und [20] und - gering abgewandelt - bei Edwards in [16. Ch. 7].

Satz 2.1.6 (Lehmersche Form der Riemann-Siegel-Formel). Für $t \rightarrow+\infty$ ist

$$
Z(t) \sim 2 \sum_{n=1}^{N} \frac{\cos (\vartheta(t)-t \log n)}{\sqrt{n}}+\frac{(-1)^{N-1}}{\sqrt{a}} \sum_{n=0}^{\infty} \frac{C_{n}(z)}{a^{n}}
$$

mit

$$
a:=\sqrt{\frac{t}{2 \pi}}, \quad N:=\lfloor a\rfloor, \quad z:=1-2(a-N) .
$$

Die Koeffizienten $C_{n}(z)$ sind mit den Zahlen $d_{k}^{(n)}$ aus Satz 2.1.1 und der Funktion

$$
F(z):=\frac{\cos \frac{\pi}{2}\left(z^{2}+\frac{3}{4}\right)}{\cos \pi z}
$$

durch

$$
C_{n}(z):=\frac{1}{2^{2 n}} \sum_{k=0}^{\lfloor 3 n / 4\rfloor} \frac{d_{k}^{(n)}}{\pi^{2 n-2 k}(3 n-4 k) !} F^{(3 n-4 k)}(z) \quad(n \geq 0)
$$

gegeben. Für die Werte der Koeffizienten $C_{n}(z)$ mit geradem Index an der Stelle 1 gilt

$$
\begin{aligned}
C_{4 n}(1) & =(-1)^{n} \frac{\alpha_{2 n}}{2^{8 n} \pi^{2 n}} \cos \frac{\pi}{8}, \\
C_{4 n+2}(1) & =(-1)^{n+1} \frac{\alpha_{2 n+1}}{2^{8 n+4} \pi^{2 n+1}} \sin \frac{\pi}{8} . \quad(n \geq 0)
\end{aligned}
$$

Beweis. Wir setzen

$$
F(z):=\widehat{F}(q)
$$

mit

$$
z:=\frac{q}{\sqrt{\pi}}
$$

7) Die $d_{k}^{(n)}$ mit ungeradem $n$ können mit diesem Verfahren zwar nicht direkt überprüft werden; man kann aber davon ausgehen, daß die $d_{k}^{(2 n-1)}$ richtig sind, wenn das für die $d_{k}^{(2 n)}$ zutrifft, da letztere aus den ersten mit Hilfe der Rekursionsformel [2.2] berechnet wurden. 
Dann gilt für $k \geq 0$

$$
\widehat{F}^{(k)}(q)=(\sqrt{\pi})^{-k} F^{(k)}(z)
$$

und 2.26) folgt wegen $F(z)=\widehat{F}(\sqrt{\pi} z)$ aus der in Satz 2.1.1 angegebenen Darstellung von $\widehat{F}(q)$.

Betrachten wir nun unsere Form der Riemann-Siegel-Formel in Satz 1.3.1. S. 18. Wegen $\tau=1 /(4 a \sqrt{\pi})$ können wir schreiben

$$
\sum_{n=0}^{\infty} \widehat{C}_{n}(q) \tau^{n} \doteq \sum_{n=0}^{\infty} \frac{\widehat{C}_{n}(q)}{(4 \sqrt{\pi})^{n} a^{n}} \doteq \sum_{n=0}^{\infty} \frac{C_{n}(z)}{a^{n}}
$$

mit

$$
C_{n}(z):=\frac{\widehat{C}_{n}(q)}{(4 \sqrt{\pi})^{n}} \quad(n \geq 0)
$$

Tragen wir hier die Darstellung (2.1) der Koeffizienten $\widehat{C}_{n}(q)$ ein und ersetzen die darin auftretenden Ableitungen von $\widehat{F}(q)$ nach Gleichung 2.29 mit denen von $F(z)$, so ergibt sich 2.27). Unter Berücksichtigung von 2.30 folgt so die in diesem Satz angegebene Form der Riemann-Siegel-Formel.

Mit $z=1$ lautet 2.31

$$
C_{n}(1)=\frac{\widehat{C}_{n}(\sqrt{\pi})}{(4 \sqrt{\pi})^{n}} \quad(n \geq 0)
$$

und die Formeln 2.28 ergeben sich aus 2.24 .

Da sich die Fakultäten sehr leicht in Primfaktoren zerlegen lassen, kann man mit Hilfe der Primfaktorzerlegung der Zahlen $d_{k}^{(n)}$ aus Tabelle II die in 2.27) auftretenden rationalen Zahlen $d_{k}^{(n)} /\left[2^{2 n}(3 n-4 k) !\right]$ fast ohne Rechnung in reduzierter Form angeben. Die sich daraus ergebenden expliziten Darstellungen der Koeffizienten $C_{n}(z)$ als Kombination von Ableitungen der Funktion $F(z)$ findet man für $n \leq 12$ in Tabelle III, S. 97 .

Für $n \leq 4$ sind die $C_{n}(z)$ auch von Haselgrove in [20] und - gering abgewandelt - von Edwards in [16. S. 154], sowie für $n \leq 8$ in der nicht reduzierten Form 2.27] von Crary und Rosser in [15] angegeben worden. In anderer Form findet man die Koeffizienten der Riemann-Siegel-Formel für $n \leq 4$ auch bei Siegel in 32 . S. 290].

Die Darstellung 2.27) der Koeffizienten $C_{n}(z)$ ist zusammen mit der Rekursionsformel (2.2) der Zahlen $d_{k}^{(n)}$ und den numerischen Werten der ersten $\lambda_{n}$ bereits in der Zeitschrift Math. of Comp. 31, No. 139, July 1977, P. 803 in Form einer Mitteilung veröffentlicht worden. Der zugehörige Beweis, den wir in diesem Abschnitt geführt haben, ist aber nach Wissen des Autors bisher noch nicht erschienen.

\subsection{Potenz- und T-Reihen der Koeffizienten $C_{n}(z)$}

Wie die Restabschätzung im nächsten Kapitel ergeben wird, kann man die Funktion $Z(t)$ mit Hilfe der Riemann-Siegel-Formel sehr genau berechnen. Verwendet 
man dazu - wie heute allgemein üblich - die Lehmersche Form aus Satz 2.1.6, so erhebt sich die Frage nach einem möglichst effektiven Verfahren zur numerischen Berechnung der Koeffizienten $C_{n}(z)$ für $|z| \leq 1$. Dafür ist nämlich

$$
C_{n}(z):=\frac{1}{2^{2 n}} \sum_{k=0}^{\lfloor 3 n / 4\rfloor} \frac{d_{k}^{(n)}}{\pi^{2 n-2 k}(3 n-4 k) !} F^{(3 n-4 k)}(z) \quad(n \geq 0)
$$

mit

$$
F(z)=\frac{\cos \frac{\pi}{2}\left(z^{2}+\frac{3}{4}\right)}{\cos \pi z}
$$

aus zwei Gründen denkbar ungeeignet: erstens, weil die expliziten Darstellungen der höheren Ableitungen von $F(z)$ eine sehr komplizierte Gestalt annehmen, und zweitens, weil die Berechnung der Funktion $F(z)$ mit 2.33 in der Nähe der Punkte $z=-1 / 2$ und $z=1 / 2$ numerisch instabil ist, da dort Zähler und Nenner gleichzeitig verschwinden. Eine einfache Überlegung zeigt, daß die letzte Bemerkung auch für die Ableitungen von $F(z)$ gilt, wenn man zu ihrer Berechnung die eben betrachteten expliziten Darstellungen heranzieht. Wir können diese Schwierigkeiten aber auf folgende Weise umgehen: Als Funktion der komplexen Veränderlichen $z$ ist $F(z)$ ganz ${ }^{8)}$ Da $F(z)$ außerdem gerade in $z$ ist, lassen sich die Funktionen $C_{n}(z)$ nach 2.32 in Potenzreihen der Form

$$
\begin{aligned}
C_{2 n}(z) & =\sum_{k=0}^{\infty} c_{2 k}^{(2 n)} z^{2 k}, \\
C_{2 n+1}(z) & =\sum_{k=0}^{\infty} c_{2 k+1}^{(2 n+1)} z^{2 k+1}
\end{aligned}
$$

entwickeln, die für alle komplexen $z$ konvergieren und deren Koeffizienten reell sind. Setzen wir in 2.34 $n=0$ und vergleichen mit 2.33, so ergibt sich wegen $C_{0}(z)=F(z)$ bei entsprechender Zerlegung von $\cos \left[\pi\left(z^{2}+3 / 4\right) / 2\right]$ die Beziehung

$$
\sin \frac{\pi}{8} \cos \frac{\pi}{2} z^{2}-\cos \frac{\pi}{8} \sin \frac{\pi}{2} z^{2}=\cos \pi z \sum_{k=0}^{\infty} c_{2 k}^{(0)} z^{2 k},
$$

aus der sich die Zahlen $c_{2 k}^{(0)}$ rekursiv berechnen lassen, wenn man die hier auftretenden trigonometrischen Funktionen mit ihren Potenzreihen um den Punkt $z=0$ ersetzt. Mit Hilfe dieser Rekursionsformel hat der Autor auf der UNIVAC 1108 der Gesellschaft für Wissenschaftliche Datenverarbeitung Göttingen unter Verwendung einer 150-stelligen dezimalen Arithmetik die ersten $c_{2 k}^{(0)}$ bis einschließlich $c_{154}^{(0)}$ mit einem absoluten Fehler $<10^{-100}$ numerisch bestimmt 9 Die Potenzreihenkoeffizienten der Ableitungen $F^{(m)}(z)$ ergaben sich hieraus für $m \leq 30$ mit so hoher relativer Genauigkeit, daß die Koeffizienten $c_{2 k}^{(10)}$ der Funktion $C_{10}(z)$, in der $F^{(30)}(z)$ auftritt, noch mit einem absoluten Fehler $<10^{-60}$ aus 2.32 berechnet

8) Das folgt entweder direkt aus [2.33], wenn man beachtet, daß alle Nullstellen von $\cos \pi z$ auch Nullstellen von $\cos \left[\pi\left(z^{2}+3 / 4\right) / 2\right]$ sind, oder aus Satz 4.1.2, S. 61 zusammen mit den Beziehungen $\widetilde{F}(q)=\widehat{F}(q)+i \widehat{F}(q)$ und $F(z)=\widehat{F}(\sqrt{\pi} z)$.

9) Die Tabellen im Anhang wurden mit selbstgeschriebenen C-Programmen neu berechnet. 
werden konnten. Für $n<10$ waren die absoluten Fehler der Potenzreihenkoeffizienten von $C_{n}(z)$ entsprechend kleiner und ließen sich deshalb ebenfalls mit $10^{-60}$ nach oben abschätzen. Auf 50 Dezimalstellen gerundete Werte dieser Potenzreihenkoeffizienten sind für $0 \leq n \leq 10$ in Tabelle IV, S. 101 abgedruckt ${ }^{10}$. Für jede dieser 11 Potenzreihen ist die Summe über alle abgedruckten Koeffizienten unterhalb der durchgezogenen Linie angegeben. Diese Summen, die Näherungen für die Funktionswerte $C_{n}(1)$ darstellen, wurden mit genaueren und auf anderem Wege berechneten Werten von $C_{n}(1)$ verglichen. In allen Fällen ergaben sich nur geringe Abweichungen in der 50-ten Stelle. Man kann daher davon ausgehen, daß sämtliche in Tabelle IV wiedergegebenen Koeffizienten korrekt auf 50 Dezimalstellen gerundet sind. Für gerades $n$ erhält man die für diesen Vergleich notwendigen genauen Werte von $C_{n}(1)$ aus (2.28). Für ungerades $n$ kann man für $C_{n}(1)$ zwar auch eine Formel angeben; da diese jedoch sehr kompliziert ist, verzichten wir darauf, sie hier wiederzugeben und verweisen auf [15]. Abschließend sei noch angemerkt, daß nicht Gleichung 2.32) sondern die reduzierten Darstellungen aus Tabelle III, S. 97 Grundlage für die Berechnungen waren.

Schon bei Lehmer finden sich in [22] erste numerische Werte der Koeffizienten der Potenzreihenentwicklungen 2.34 und 2.35. Haselgrove gibt diese Koeffizienten für $n \leq 4$ auf etwa 11 bis 20 Dezimalstellen genau in [20] an. Die von ihm gefundenen Werte sind von Edwards in [16. S. 158] übernommen worden. 70-stellige Werte, die mit denen aus Tabelle IV sehr gut übereinstimmen, haben Crary und Rosser für $n \leq 6$ in [15] angegeben; jedoch sind dort nicht alle Koeffizienten aufgeführt, deren Betrag $>10^{-70}$ ist. Die in Tabelle IV wiedergegebenen Potenzreihenkoeffizienten von $C_{7}(z)$ bis $C_{10}(z)$ dürften neu sein.

Mit Hilfe dieser Potenzreihen kann man die Funktionen $C_{n}(z)$ bereits auf recht einfache Weise mit hoher Genauigkeit berechnen. Jedoch sind gerade für numerische Zwecke Entwicklungen Tschebyscheffschen Polynomen erster Art ${ }^{11)}$ yesentlich günstiger. Für reelle $z$ mit $|z| \leq 1$ sind diese Polynome durch

$$
T_{k}(z):=\cos (k \arccos z) \quad(k \geq 0)
$$

10) Hinweis für den an einer eigenen Berechnung interessierten Leser

Die Rekursionsformel 2.36 ist numerisch instabil, so daß ihre Auswertung mit einer erheblich höheren Rechengenauigkeit vorgenommen werden muß, als die gewünschte Genauigkeit der Potenzreihenkoeffizienten beträgt. Diese numerische Instabilität ist ein wesentliches Merkmal der Rekursionsformel, das sich nicht - etwa durch die Art der verwendeten Arithmetik (Fest- oder Gleitpunktarithmetik), die Art und Weise ihrer Implementierung oder ähnlicher Kriterien - beeinflussen läßt. Ursache dafür ist die unterschiedliche Geschwindigkeit, mit der die Potenzreihenkoeffizienten von $\cos \left(\pi z^{2} / 2\right)$ bzw. $\sin \left(\pi z^{2} / 2\right)$ im Vergleich zu denen von $\cos \pi z$ gegen Null gehen.

Die bei vorgegebener Rechengenauigkeit maximal erreichbare Genauigkeit der Potenzreihenkoeffizienten läßt sich auf folgende Weise experimentell bestimmen:

Man drucke die Koeffizienten mit der vollen Rechengenauigkeit so lange aus, bis die Beträge dieser Koeffizienten nicht mehr weiter zurückgehen, sondern aufgrund der endlichen Rechengenauigkeit und der daraus resultierenden Rundungsfehler wieder zu steigen beginnen. Dann liegt die maximal erreichbare Genauigkeit in der Größenordnung des betragskleinsten Koeffizienten und alle ab diesem Koeffizienten noch weiter berechneten Potenzreihenkoeffizienten sowie die Dezimal- oder Binärstellen ab dieser Größenordnung in den vorangehenden Koeffizienten sind irrelevant. Sollte die so bestimmte maximale Genauigkeit nicht ausreichend sein, ist eine Erhöhung der Rechengenauigkeit nicht zu vermeiden.

11) Eine Aufstellung der Eigenschaften dieser Polynome findet man z. B. in [1. Kap. 22]. 
gegeben. Sie genügen der Rekursionsformel

$$
\begin{gathered}
T_{0}(z) \equiv 1, \\
T_{1}(z)=z, \\
T_{k+1}(z)=2 z T_{k}(z)-T_{k-1}(z) \quad(k \geq 1) .
\end{gathered}
$$

Wir haben die geraden Funktionen $C_{2 n}(z)$ und $z^{-1} C_{2 n+1}(z)$ in Reihen nach geraden Tschebyscheffpolynomen entwickelt

$$
\begin{array}{rlr}
C_{2 n}(z) & =\sum_{k=0}^{\infty} \gamma_{2 k}^{(2 n)} T_{2 k}(z), \quad(n \geq 0) \\
C_{2 n+1}(z) & =z \sum_{k=0}^{\infty} \gamma_{2 k}^{(2 n+1)} T_{2 k}(z), &
\end{array}
$$

die wie die entsprechenden Potenzreihen für alle komplexen $z$ konvergieren. Dabei bedeutet der Strich an den Summenzeichen, daß das konstante Glied - wie bei Fourierreihen üblich ${ }^{12)}$ zu halbieren ist.

Die Koeffizienten $\gamma_{2 k}^{(n)}$ kann man mit Hilfe der Formeln

$$
\begin{aligned}
\gamma_{2 k}^{(2 n)} & =\sum_{l=k}^{\infty}\left(\begin{array}{c}
2 l \\
l-k
\end{array}\right) \frac{c_{2 l}^{(2 n)}}{2^{2 l-1}}, \quad(n \geq 0, k \geq 0) \\
\gamma_{2 k}^{(2 n+1)} & =\sum_{l=k}^{\infty}\left(\begin{array}{c}
2 l \\
l-k
\end{array}\right) \frac{c_{2 l+1}^{(2 n+1)}}{2^{2 l-1}}
\end{aligned}
$$

aus den Potenzreihenkoeffizienten $c_{k}^{(n)}$ berechnen. Man erhält diese Formeln, wenn man die Tschebyscheffentwicklung der geraden $z$-Potenzen

$$
z^{2 k}=\sum_{l=0}^{k} 2^{-2 k+1}\left(\begin{array}{c}
2 k \\
k-l
\end{array}\right) T_{2 l}(z) \quad(k \geq 0)
$$

in die Potenzreihenentwicklungen 2.34 und 2.35) einsetzt und die dabei entstehenden unendlichen Reihen aufsteigend nach Tschebyscheffschen Polynomen anordnet. Mit den Formeln (2.39) hat der Autor die $\gamma_{2 k}^{(n)}$ für $n \leq 10$ auf der oben genannten Rechenanlage aus den 60-stelligen Werten der Potenzreihenkoeffizienten numerisch bestimmt. Die auf 50 Dezimalstellen gerundeten Werte finden sich in Tabelle V, S. 113. Zur Kontrolle sind auch hier für jede der 11 Tschebyscheffreihen die Summen über alle in der Tabelle aufgeführten Koeffizienten - bei Halbierung des konstanten Gliedes - unterhalb der durchgezogenen Linie angegeben. Diese Summen stellen ebenfalls Näherungen für die Werte $C_{n}(1)$ dar; denn wegen $T_{k}(1)=1$ für $k \geq 0$ folgt aus den Formeln 2.38 $C_{n}(1)=\sum_{k=0}^{\prime \infty} \gamma_{2 k}^{(n)}$. Ein Vergleich mit den entsprechenden Summen von Tabelle IV ergibt nur geringe Abweichungen in

12) Mit der Substitution $x=\cos \theta$ geht nämlich eine Tschebyscheffreihe der Form $f(x)=$ $\sum_{k=0}^{\prime \infty} a_{k} T_{k}(x)$ wegen 2.37 in die Fouriercosinusreihe $f(\cos \theta)=\sum_{k=0}^{\prime \infty} a_{k} \cos k \theta$ über. 
der 50-ten Dezimalstelle. Man kann daher auch hier davon ausgehen, daß alle in Tabelle V angegebenen Koeffizienten korrekt auf 50 Dezimalstellen gerundet sind.

In der Lehmerschen Form der Riemann-Siegel-Formel (Satz 2.1.6 benötigen wir die Koeffizienten $C_{n}(z)$ nur für reelle $z$ mit $|z| \leq 1{ }^{13)}$ Aus numerischer Sicht sind aber gerade für diese $z$ die Tschebyscheffentwicklungen besonders gut geeignet, da die Tschebyscheffschen Polynome in diesem Intervall der Abschätzung $\left|T_{k}(z)\right| \leq 1$ genügen. Anhand des folgenden Beispiels soll der Vorteil der Tschebyscheffentwicklungen gegenüber den entsprechenden Potenzreihenentwicklungen deutlich gemacht werden.

Für reelle $z$ mit $|z| \leq 1$ ist eine Polynomapproximation für $C_{10}(z)$ gesucht, deren absoluter Fehler $<10^{-12}$ ist. Der erste Koeffizient in der Potenzreihe von $C_{10}(z)$, den man nach Tabelle IV, S. 112 vernachlässigen darf, ist $c_{38}^{(10)}$, so daß sich ein Approximationspolynom vom Grade 18 in $z^{2}$ ergibt. Verwendet man jedoch die Tschebyscheffentwicklung von $C_{10}(z)$, so zeigt Tabelle V, S. 124, daß wegen $\left|T_{k}(z)\right| \leq 1$ alle Koeffizienten ab $\gamma_{20}^{(10)}$ vernachlässigt werden können. Das auf diese Weise entstehende Polynom hat dann nur den Grad 9 in $z^{2}$ und ist wegen des geringeren Rechenaufwandes für numerische Zwecke wesentlich besser geeignet als das aus der Potenzreihe gewonnene Approximationspolynom. Wenn man die Tschebyscheffentwicklungen 2.38 verwendet, bereitet die Berechnung der Funktionen $C_{n}(z)$ daher keinerlei Schwierigkeiten mehr.

\subsection{Abschätzungen von $C_{n}(z)(0 \leq n \leq 10,|z| \leq 1)$}

Wir wollen jetzt den Fehler untersuchen, den man begeht, wenn man die Potenzreihen 2.34 und 2.35 nach dem K-ten Gliede abbricht. Dazu benötigen wir Abschätzungen für die Ableitungen der Funktion $F(z)$, die man aus dem folgenden Satz erhält.

Satz 2.3.1. Für $n \geq 0$ und reelle $z$ mit $|z| \leq 1$ gelten die Abschätzungen

$$
\begin{gathered}
\left|F^{(2 n)}(z)\right| \leq \frac{(2 n) !}{2^{n} n !} \pi^{n}, \\
\left|F^{(2 n+1)}(z)\right| \leq 2^{n+1} \pi^{n} n ! .
\end{gathered}
$$

Insbesondere genügen die Potenzreihenkoeffizienten $c_{2 k}^{(0)}$ von $F(z)$ der Abschätzung ${ }^{14)}$

$$
\left|c_{2 k}^{(0)}\right| \leq \frac{\pi^{k}}{2^{k} k !} \quad(k \geq 0) .
$$

Beweis. Aus der Integraldarstellung von $F(z)$ (Satz 4.1.4. S. 64 folgt durch $2 n$ bzw. $2 n+1$-malige Differentiation nach $z$

$$
F^{(2 n)}(z)=\sqrt{2} \Re\left(e^{-i \pi / 8} \int_{0}^{\infty} e^{-\pi v^{2} / 2}\left(\pi e^{i \pi / 4} v\right)^{2 n} \frac{\cosh \left(\pi z e^{i \pi / 4} v\right)}{\cosh \left(\pi e^{i \pi / 4} v\right)} d v\right),
$$

13) In Satz 2.1.6 ist zwar immer $z>-1$; da $z$ dem Wert -1 aber beliebig nahe kommen kann, ist es sinnvoll, diesen in die Betrachtung mit aufzunehmen.

14) Vgl. hierzu die in [15] gefundene, wesentlich schlechtere Abschätzung $\left|c_{2 k}^{(0)}\right|<c \cdot 5^{-2 k}$. Dabei ist $c$ eine absolute positive Konstante. 


$$
F^{(2 n+1)}(z)=\sqrt{2} \Re\left(e^{-i \pi / 8} \int_{0}^{\infty} e^{-\pi v^{2} / 2}\left(\pi e^{i \pi / 4} v\right)^{2 n+1} \frac{\sinh \left(\pi z e^{i \pi / 4} v\right)}{\cosh \left(\pi e^{i \pi / 4} v\right)} d v\right) .
$$

Unter Verwendung des Satzes 4.4.1, S. 80 gelten dann für reelle $z$ mit $|z| \leq 1$ die Abschätzungen

$$
\begin{gathered}
\left|F^{(2 n)}(z)\right| \leq \sqrt{2} \int_{0}^{\infty} e^{-\pi v^{2} / 2}(\pi v)^{2 n} d v=\frac{(2 \pi)^{n}}{\sqrt{\pi}} \Gamma\left(n+\frac{1}{2}\right), \\
\left|F^{(2 n+1)}(z)\right| \leq \sqrt{2} \int_{0}^{\infty} e^{-\pi v^{2} / 2}(\pi v)^{2 n+1} \sqrt{2} d v=2^{n+1} \pi^{n} \Gamma(n+1),
\end{gathered}
$$

aus denen 2.40 und 2.41 folgen, wenn man

$$
\Gamma\left(n+\frac{1}{2}\right)=\frac{(2 n) !}{2^{2 n} n !} \sqrt{\pi}
$$

beachtet. Die Abschätzung der Potenzreihenkoeffizienten $c_{2 k}^{(0)}=F^{(2 k)}(0) /(2 k)$ ! erhält man direkt aus 2.40.

In der Lehmerschen Form der Riemann-Siegel-Formel ist $z$ reell mit $|z| \leq 1$ 15) In allen jetzt folgenden Abschätzungen bis zum Ende dieses Abschnittes sind daher immer diese $z$ gemeint, auch wenn das nicht ausdrücklich erwähnt wird.

Mit dem Restglied von Lagrange ergeben sich aus 2.34 und 2.35 für $K \geq 0$ die Gleichungen

$$
\begin{aligned}
C_{2 n}(z) & =\sum_{k=0}^{K} c_{2 k}^{(2 n)} z^{2 k}+\frac{C_{2 n}^{(2 K+2)}(\xi)}{(2 K+2) !} z^{2 K+2}, \quad(n \geq 0) \\
C_{2 n+1}(z) & =\sum_{k=0}^{K} c_{2 k+1}^{(2 n+1)} z^{2 k+1}+\frac{C_{2 n+1}^{(2 K+3)}(\xi)}{(2 K+3) !} z^{2 K+3} .
\end{aligned}
$$

Dabei ist $\xi$ eine reelle Zahl, die wegen $|z| \leq 1$ der Ungleichung $|\xi|<1$ genügt. Die Restglieder in 2.42 lassen sich dann folgendermaßen abschätzen:

Satz 2.3.2. Für $|\xi|<1$ und $K \geq 0$ gelten die Abschätzungen

$$
\begin{aligned}
& \frac{\left|C_{2 n}^{(2 K+2)}(\xi)\right|}{(2 K+2) !} \leq \frac{\pi^{K+1-n}}{2^{7 n+K+1}} \sum_{k=0}^{\left\lfloor\frac{3 n}{2}\right\rfloor}\left(\begin{array}{c}
6 n+2 K+2-4 k \\
2 K+2
\end{array}\right) \frac{2^{2 k} d_{k}^{(2 n)}}{(3 n+K+1-2 k) !}, \\
& \frac{\left|C_{2 n+1}^{(2 K+3)}(\xi)\right|}{(2 K+3) !} \leq \frac{\pi^{K+1-n}}{2^{7 n+K+5}} \sum_{k=0}^{\left\lfloor\frac{6 n+3}{4}\right\rfloor}\left(\begin{array}{c}
6 n+2 K+6-4 k \\
2 K+3
\end{array}\right) \frac{2^{2 k} d_{k}^{(2 n+1)}}{(3 n+K+3-2 k) !} .
\end{aligned}
$$

15) Vgl. dazu die Fußnote 13) auf Seite 35. 
Beweis. Wir ersetzen $n$ in 2.32 mit $2 n$ bzw. $2 n+1$. Dann wird

$$
\begin{aligned}
& \frac{\left|C_{2 n}^{(2 K+2)}(\xi)\right|}{(2 K+2) !}=\frac{1}{2^{4 n}(2 K+2) !} \sum_{k=0}^{\left\lfloor\frac{3 n}{2}\right\rfloor} \frac{d_{k}^{(2 n)}}{\pi^{4 n-2 k}(6 n-4 k) !} F^{(6 n+2 K+2-4 k)}(\xi), \\
& \frac{\left|C_{2 n+1}^{(2 K+3)}(\xi)\right|}{(2 K+3) !}=\frac{1}{2^{4 n+2}(2 K+3) !} \sum_{k=0}^{\left\lfloor\frac{6 n+3}{4}\right\rfloor} \frac{d_{k}^{(2 n+1)}}{\pi^{4 n+2-2 k}(6 n+3-4 k) !} F^{(6 n+2 K+6-4 k)}(\xi),
\end{aligned}
$$

und hieraus folgt nach einfacher Umformung die Behauptung des Satzes, wenn man die Ableitungen von $F(\xi)$ mit ihren Abschätzungen aus Satz 2.3.1 ersetzt. Dazu benötigt man übrigens nur 2.40, da alle hier auftretenden Ableitungen von $F(\xi)$ gerade sind.

Für $0 \leq n \leq 10$ können wir jetzt den Fehler abschätzen, der entsteht, wenn man die Funktionen $C_{n}(z)$ aus ihren Potenzreihenentwicklungen unter Berücksichtigung aller in Tabelle IV wiedergegebenen Koeffizienten berechnet. Dazu wählen wir die Werte von $\mathrm{K}$ in den Formeln 2.42 so, daß $c_{2 K}^{(2 n)}$ bzw. $c_{2 K+1}^{(2 n+1)}$ die letzten noch in Tabelle IV wiedergegebenen Koeffizienten werden. Auf die sich so ergebenden Restglieder wenden wir Satz 2.3.2 an und erhalten, wenn wir die dort auftretenden Summen mit den exakten Werten der Zahlen $d_{k}^{(n)}$ aus Tabelle II, S. 95 entsprechend genau abschätzen:

Satz 2.3.3. Für $|\xi|<1$ ist

$$
\begin{array}{rlr}
\frac{\left|C_{0}^{(88)}(\xi)\right|}{88 !}=\frac{\left|F^{(88)}(\xi)\right|}{88 !}<1.7 \cdot 10^{-46}, \\
\frac{\left|C_{1}^{(91)}(\xi)\right|}{91 !}<4.4 \cdot 10^{-47}, & \frac{\left|C_{6}^{(98)}(\xi)\right|}{98 !}<2.0 \cdot 10^{-46}, \\
\frac{\left|C_{2}^{(92)}(\xi)\right|}{92 !}<1.9 \cdot 10^{-46}, & \frac{\left|C_{7}^{(99)}(\xi)\right|}{99 !}<3.5 \cdot 10^{-46}, \\
\frac{\left|C_{3}^{(93)}(\xi)\right|}{93 !}<5.5 \cdot 10^{-46}, & \frac{\left|C_{8}^{(100)}(\xi)\right|}{100 !}<5.6 \cdot 10^{-46}, \\
\frac{\left|C_{4}^{(94)}(\xi)\right|}{94 !}<1.4 \cdot 10^{-45}, & \frac{\left|C_{9}^{(101)}(\xi)\right|}{101 !}<8.5 \cdot 10^{-46}, \\
\frac{\left|C_{5}^{(95)}(\xi)\right|}{95 !}<2.8 \cdot 10^{-45}, & \frac{\left|C_{10}^{(102)}(\xi)\right|}{102 !}<1.3 \cdot 10^{-45} .
\end{array}
$$

Mit diesen Abschätzungen folgt aus (2.42), daß man $C_{n}(z)$ für $0 \leq n \leq 10$ und $|z| \leq 1$ auf mindestens 44 Dezimalstellen genau berechnen kann, wenn man alle in Tabelle IV abgedruckten Potenzreihenkoeffizienten berücksichtigt. Verglichen mit diesen Abschätzungen sind natürlich die Fehler, die durch das Runden der Potenzreihenkoeffizienten auf 50 Dezimalstellen entstehen, vernachlässigbar klein, so daß wir auf ihre Abschätzung verzichten können.

Zur Genauigkeit der Tschebyscheffentwicklungen bemerken wir noch, daß der Fehler, der entsteht, wenn man die unendliche Reihe $\sum_{k=0}^{\infty} \gamma_{2 k}^{(n)} T_{2 k}(z)$ mit der 
Partialsumme $\sum_{k=0}^{K^{\prime}} \gamma_{2 k}^{(n)} T_{2 k}(z)$ ersetzt, für alle $z$ mit $|z| \leq 1$ dem Betrage nach nur wenig größer ist als der Betrag des ersten vernachlässigten Koeffizienten $\gamma_{2 K+2}^{(n)}$. Auf eine genauere Untersuchung dieser Fehler können wir hier aber nicht eingehen.

Der folgende Satz gibt Abschätzungen für die Funktionen $C_{n}(z)$.

Satz 2.3.4. Für $0 \leq n \leq 10$ und $|z| \leq 1$ lassen sich die Funktionen $C_{n}(z)$

(a) ohne Verwendung ihrer Potenzreihenentwicklungen mit

$$
\begin{array}{rlrl}
\qquad C_{0}(z)|=| F(z) \mid & \leq 1, \\
\left|C_{1}(z)\right| & <1.1 \cdot 10^{-1}, & & \left|C_{6}(z)\right|<6.7 \cdot 10^{-3}, \\
\left|C_{2}(z)\right| & <3.7 \cdot 10^{-2}, & & \left|C_{7}(z)\right|<8.3 \cdot 10^{-3} \\
\left|C_{3}(z)\right| & <2.8 \cdot 10^{-2}, & & \left|C_{8}(z)\right|<5.6 \cdot 10^{-3} \\
\left|C_{4}(z)\right| & <1.2 \cdot 10^{-2}, & & \left|C_{9}(z)\right|<8.2 \cdot 10^{-3} \\
\left|C_{5}(z)\right| & <1.2 \cdot 10^{-2}, & & \left|C_{10}(z)\right|<6.3 \cdot 10^{-3}
\end{array}
$$

grob abschätzen.

(b) Numerische Untersuchungen ihrer Potenzreihen führen zu den optimalen Abschätzungen

$$
\begin{array}{rlrl}
\left|C_{0}(z)\right|=|F(z)|<9.3 \cdot 10^{-1}, \\
\left|C_{1}(z)\right|<3.1 \cdot 10^{-2}, & \left|C_{6}(z)\right|<3.4 \cdot 10^{-5}, \\
\left|C_{2}(z)\right|<5.2 \cdot 10^{-3}, & \left|C_{7}(z)\right|<1.1 \cdot 10^{-5}, \\
\left|C_{3}(z)\right|<3.2 \cdot 10^{-4}, & \left|C_{8}(z)\right|<2.5 \cdot 10^{-6}, \\
\left|C_{4}(z)\right|<4.7 \cdot 10^{-4}, & \left|C_{9}(z)\right|<2.5 \cdot 10^{-6}, \\
\left|C_{5}(z)\right|<7.6 \cdot 10^{-5}, & \left|C_{10}(z)\right|<2.2 \cdot 10^{-7} .
\end{array}
$$

Beweis. Wir ersetzen in 2.32 wieder $n$ mit $2 n$ bzw. $2 n+1$. Mit Hilfe von Satz 2.3 .1 wird dann

$$
\begin{gathered}
\left|C_{2 n}(z)\right| \leq \frac{1}{2^{7 n} \pi^{n}} \sum_{k=0}^{\left\lfloor\frac{3 n}{2}\right\rfloor} \frac{2^{2 k} d_{k}^{(2 n)}}{(3 n-2 k) !}, \quad(n \geq 0) \\
\left|C_{2 n+1}(z)\right| \leq \frac{1}{2^{n} \pi^{n+1}} \sum_{k=0}^{\left\lfloor\frac{6 n+3}{4}\right\rfloor} \frac{d_{k}^{(2 n+1)}(3 n+1-2 k) !}{2^{2 k}(6 n+3-4 k) !},
\end{gathered}
$$

und hieraus erhält man (a), wenn man diese Summen mit den Zahlen $d_{k}^{(n)}$ aus Tabelle II mit der entsprechenden Genauigkeit abschätzt.

Die optimalen Abschätzungen (b) ergeben sich durch genauere numerische Untersuchungen der Funktionen $C_{n}(z)$ mit Hilfe ihrer Potenzreihen unter Verwendung von Tabelle IV. Aufgrund der Ergebnisse von Satz 2.3.3 ist dieses Vorgehen gerechtfertigt. 
Bei der Restabschätzung der Riemann-Siegel-Formel im nächsten Kapitel werden uns diese Abschätzungen der $C_{n}(z)$ noch von Nutzen sein. Mit Hilfe von (b) wird es gelingen, die ersten fünf Restglieder der Riemann-Siegel-Formel optimal abzuschätzen. 



\section{Kapitel 3}

\section{Die Riemann-Siegel-Formel mit Restglied}

\subsection{Restabschätzung der asymptotischen Reihe von $S$}

In Kapitel 1. S. 7 haben wir für den Ausdruck

$$
S:=\frac{1}{2 \sqrt{\pi}} e^{i \pi / 8-i q^{2} / 2} \int_{\tau^{i q}} \frac{e^{i v^{2} / 2+q v}}{\cosh \frac{\sqrt{\pi}}{2} v} g(\tau, v-i q) d v \quad \begin{aligned}
& (\tau>0, \\
& -\sqrt{\pi}<q \leq \sqrt{\pi})
\end{aligned}
$$

die formale Entwicklung nach Potenzen von $\tau$

$$
S \sim \sum_{n=0}^{\infty} B_{n}(q) \tau^{n}
$$

hergeleitet. Wir wollen jetzt untersuchen, mit welcher Genauigkeit die Partialsummen dieser formalen Reihe den Ausdruck $S$ approximieren. Da $q$ dem Wert $-\sqrt{\pi}$ beliebig nahe kommen kann, schließen wir ihn in diesem Kapitel ausdrücklich mit ein ${ }^{1)}$ und definieren die Restglieder

$$
\operatorname{RS}_{K}(\tau):=S-\sum_{n=0}^{K} B_{n}(q) \tau^{n} \quad(K \geq 0, \tau>0,|q| \leq \sqrt{\pi}),
$$

die dann für diese $K, \tau$ und $q$ allgemeingültig abgeschätzt werden müssen. Bis zum Ende dieses Abschnittes beziehen sich alle jetzt folgenden Formeln und Sätze grundsätzlich auf diese Werte von $K, \tau$ und $q$. Eventuell bei $\tau$ auftretende Betragstriche können wir wegen $\tau>0$ fortlassen. Zur Abkürzung setzen wir noch $\varepsilon:=e^{i \pi / 4}$ und geben zunächst eine exakte Darstellung für $\operatorname{RS}_{K}(\tau)$ an.

Satz 3.1.1. Die Restglieder $\operatorname{RS}_{K}(\tau)$ besitzen die Integraldarstellung

$$
\operatorname{RS}_{K}(\tau)=\frac{\varepsilon}{2 \sqrt{\pi}} e^{i \pi / 8} \int_{-\infty}^{+\infty} \frac{e^{-u^{2} / 2}}{\cosh \frac{\sqrt{\pi}}{2}(i q+\varepsilon u)} \operatorname{Rg}_{K}(\tau, \varepsilon u) d u
$$

\footnotetext{
1) In der Originalarbeit ist das noch nicht vollständig umgesetzt worden.
} 
die auch für $q= \pm \sqrt{\pi}$ gültig bleibt. Dabei sind die Funktionen $\operatorname{Rg}_{K}(\tau, z)$ die Restglieder in der Entwicklung von $g(\tau, z)$ nach Potenzen von $\tau$, die für alle $z$ in der längs der positiven imaginären Achse bis zum Punkt $z=i /(2 \tau)$ aufgeschnittenen $z$-Ebene durch

$$
\operatorname{Rg}_{K}(\tau, z):=g(\tau, z)-\sum_{n=0}^{K} P_{n}(z) \tau^{n}
$$

gegeben sind. Für diese $z$ ist

$$
\operatorname{Rg}_{K}(\tau, z)=\tau^{K+1} z \int_{0}^{1} \frac{P_{K+1}^{\prime}(z w)}{1+2 i \tau z w} \exp \left(\tau z \int_{w}^{1} \frac{(z v)^{2}-i}{1+2 i \tau z v} d v\right) d w
$$

Beweis. Da die $P_{n}(z)$ Polynome in $z$ sind, unterscheiden sich die in 3.4 eingeführten Restglieder $\operatorname{Rg}_{K}(\tau, z)$ für jedes $K \geq 0$ nur um ein Polynom von $g(\tau, z)$ und besitzen deshalb dieselben Singularitäten wie diese Funktion. Nach Definition von $g(\tau, z)$ in Satz 1.2.1. S. 12 ist $\operatorname{Rg}_{K}(\tau, z)$ daher in der längs der positiven imaginären Achse bis zum Punkt $z=i /(2 \tau)$ aufgeschnittenen $z$-Ebene eine holomorphe Funktion von $z$, die wegen $g(\tau, 0) \equiv 1, P_{0}(z) \equiv 1$ und $P_{n}(0)=0$ für $n \geq 1$ der Bedingung

$$
\operatorname{Rg}_{K}(\tau, 0) \equiv 0
$$

genügt. Folglich verschwindet $\operatorname{Rg}_{K}(\tau, z)$ für alle $K \geq 0$ bei $z=0$ von mindestens erster Ordnung. Aus (3.4) - mit $z=v-i q$ - und (3.1) folgt unter Verwendung von (1.25) durch Vergleich mit 3.2 die Darstellung

$$
\operatorname{RS}_{K}(\tau)=\frac{1}{2 \sqrt{\pi}} e^{i \pi / 8-i q^{2} / 2} \int_{\gamma^{i q}} \frac{e^{i v^{2} / 2+q v}}{\cosh \frac{\sqrt{\pi}}{2} v} \operatorname{Rg}_{K}(\tau, v-i q) d v .
$$

Da $\operatorname{Rg}_{K}(\tau, v-i q)$ bei $v=i q$ von mindestens erster Ordnung und $\cosh \sqrt{\pi} v / 2$ bei $v=i q$ von höchstens erster Ordnung verschwinden, ist hier der Integrand im Punkte $v=i q$ auch dann holomorph, wenn $q= \pm \sqrt{\pi}$ wird. Die Abänderung des Integrationsweges $\gamma^{i q}$ in (3.7) für $q=+\sqrt{\pi}$ ist also nicht mehr notwendig und kann mit Hilfe des Cauchyschen Integralsatzes rückgängig gemacht werden. Daher dürfen wir den Integrationsweg in (3.7) auch für $q= \pm \sqrt{\pi}$ mit $v=i q+\varepsilon u(-\infty<$ $u<+\infty)$ parametrisieren, woraus sich die in (3.3) angegebene Integraldarstellung von $\operatorname{RS}_{K}(\tau)$ ergibt.

Die Gleichung (3.5) erhält man in einfacher Weise aus der Differentialgleichung von $g(\tau, z)(\operatorname{vgl} .(1.19)$, S. 14). Setzen wir dort (3.4) ein, so folgt - die hochgestellten Striche bedeuten gewöhnliche bzw. partielle Differentiation nach $z-$

$$
\begin{aligned}
& \sum_{n=0}^{K} P_{n}^{\prime}(z) \tau^{n}+\sum_{n=0}^{K} 2 i z P_{n}^{\prime}(z) \tau^{n+1}-\sum_{n=0}^{K}\left(z^{2}-i\right) P_{n}(z) \tau^{n+1} \\
& \quad+(1+2 i \tau z) \operatorname{Rg}_{K}^{\prime}(\tau, z)-\tau\left(z^{2}-i\right) \operatorname{Rg}_{K}(\tau, z)=0
\end{aligned}
$$


und daraus wegen $P_{0}^{\prime}(z)=0$

$$
\begin{gathered}
\sum_{n=0}^{K}\left[P_{n+1}^{\prime}(z)+2 i z P_{n}^{\prime}(z)-\left(z^{2}-i\right) P_{n}(z)\right] \tau^{n+1}-P_{K+1}^{\prime}(z) \tau^{K+1} \\
+(1+2 i \tau z) \operatorname{Rg}_{K}^{\prime}(\tau, z)-\tau\left(z^{2}-i\right) \operatorname{Rg}_{K}(\tau, z)=0
\end{gathered}
$$

Nach 1.20 , S. 15 verschwindet hier die Summe identisch, und wir erhalten für $\operatorname{Rg}_{K}(\tau, z)$ die inhomogene lineare Differentialgleichung erster Ordnung

$$
(1+2 i \tau z) \operatorname{Rg}_{K}^{\prime}(\tau, z)-\tau\left(z^{2}-i\right) \operatorname{Rg}_{K}(\tau, z)=P_{K+1}^{\prime}(z) \tau^{K+1},
$$

die sich nur durch die Inhomogenität $P_{K+1}^{\prime}(z) \tau^{K+1}$ von der Differentialgleichung für $g(\tau, z)$ unterscheidet. Unter Berücksichtigung der Anfangsbedingung (3.6) ist daher 2$)$

$$
=\exp \left(\tau \int_{0}^{z} \frac{y^{2}-i}{1+2 i \tau y} d y\right) \int_{0}^{\operatorname{Rg}_{K}(\tau, z)}\left[\frac{P_{K+1}^{\prime}(x) \tau^{K+1}}{1+2 i \tau x} \exp \left(-\tau \int_{0}^{x} \frac{y^{2}-i}{1+2 i \tau y} d y\right)\right] d x .
$$

Dabei soll in allen drei Integralen als Integrationsweg die Verbindungsstrecke des Nullpunktes mit dem Punkt $z$ bzw. $x$ genommen werden. Für alle $z$ aus der längs der positiven imaginären Achse bis zum Punkt $z=i /(2 \tau)$ aufgeschnittenen $z$ Ebene sind dann die Ausdrücke $1+2 i \tau x$ und $1+2 i \tau y$ auf den so festgelegten Integrationswegen immer $\neq 0$, so daß die rechte Seite von 3.8 für diese $z$ eine holomorphe Funktion von $z$ ist. Damit ist durch 3.8 eine allgemeingültige Darstellung der Restglieder $\operatorname{Rg}_{K}(\tau, z)$ gegeben. Schreiben wir 3.8 in der Form

$$
\operatorname{Rg}_{K}(\tau, z)=\tau^{K+1} \int_{0}^{z} \frac{P_{K+1}^{\prime}(x)}{1+2 i \tau x} \exp \left(\tau \int_{x}^{z} \frac{y^{2}-i}{1+2 i \tau y} d y\right) d x
$$

und parametrisieren zuerst den Integrationsweg des äußeren Integrals mit $x=z w$ $(0 \leq w \leq 1)$ und danach den des inneren Integrals mit $y=z v(w \leq v \leq 1)$, so ergibt sich 3.5 und der Satz ist bewiesen.

Zur Abschätzung von $\operatorname{RS}_{K}(\tau)$ benötigen wir zunächst eine Abschätzung für $\operatorname{Rg}_{K}(\tau, z)$. Dabei können wir uns wegen (3.3) auf diejenigen $z$ beschränken, die auf der Winkelhalbierenden des ersten und dritten Quadranten liegen. Nun zeigen

2) Bekanntlich hat die inhomogene lineare Differentialgleichung erster Ordnung

$$
a(z) f^{\prime}(z)+b(z) f(z)=h(z) \quad a(z) \not \equiv 0
$$

die allgemeine Lösung

$$
f(z)=\exp \left(-\int \frac{b(z)}{a(z)} d z\right)\left\{\int\left[\frac{h(z)}{a(z)} \exp \left(\int \frac{b(z)}{a(z)} d z\right)\right] d z+\text { const. }\right\} .
$$

Da $g(\tau, z)$ Lösung der homogenen Differentialgleichung ist, könnte man 3.8 auch mit Hilfe von $g(\tau, z)$ schreiben. Für die Abschätzung von $\operatorname{Rg}_{K}(\tau, z)$ im nächsten Satz ist das aber nicht vorteilhaft. 
bereits ganz grobe Abschätzungen von 3.5 mit $z=\varepsilon u$ und reellem $u$, daß für $\operatorname{Rg}_{K}(\tau, \varepsilon u)$ eine Abschätzung der Form

$$
\left|\operatorname{Rg}_{K}(\tau, \varepsilon u)\right| \leq \mid \text { Polynom in } u \mid e^{c u^{2}} \tau^{K+1}
$$

mit einer positiven Konstanten $c$ zu erwarten ist. Schätzt man hiermit das Integral in $(3.3$ ab, so muß $c$ der Bedingung $c \leq 1 / 2$ genügen, damit das Integral konvergiert. Die Abschätzung von $\operatorname{Rg}_{K}(\tau, \varepsilon u)$ ist daher mit der entsprechenden Sorgfalt vorzunehmen. In dem jetzt folgenden Satz haben wir das berücksichtigt.

Satz 3.1.2. Für reelle u gelten die Abschätzungen

$$
\left|\operatorname{Rg}_{K}(\tau, \varepsilon u)\right|<\left\{\begin{aligned}
e^{u^{2} / 4} V_{K}(u) \tau^{K+1} & \text { für } u \leq-\frac{\theta}{2 \sqrt{2} \tau}, \\
e^{\omega u^{2} / 4} V_{K}(u) \tau^{K+1} & \text { für }-\frac{\theta}{2 \sqrt{2} \tau}<u<0, \\
\sqrt{2} V_{K}(u) \tau^{K+1} & \text { für } 0 \leq u \leq \frac{1}{2 \sqrt{2} \tau}, \\
\sqrt{2} e^{0.28 u^{2}} V_{K}(u) \tau^{K+1} & \text { für } u>\frac{1}{2 \sqrt{2} \tau} .
\end{aligned}\right.
$$

Dabei ist $V_{K}(u)$ mit den natürlichen Zahlen $a_{k}^{(n)}$ aus 1.23, S. 16 durch

$$
V_{K}(u):=\sum_{k=0}^{K+1} \frac{a_{k}^{(K+1)}}{(K+1+2 k) !}|u|^{K+1+2 k}
$$

und $\omega$ für beliebige reelle $\theta>0$ durch

$$
\omega:=1-\frac{2}{\theta}+\frac{4}{\theta^{2}} \arctan \frac{\theta}{\theta+2}
$$

gegeben, wobei arctan die gewöhnliche reelle Arkustangensfunktion ist. Für diese $\theta$ ist stets $0<\omega<1$.

Beweis. Mit $z=\varepsilon u$ folgt aus 3.5

$$
\operatorname{Rg}_{K}(\tau, \varepsilon u)=\tau^{K+1} i u \int_{0}^{1} \frac{P_{K+1}^{\prime}(\varepsilon u w)}{\varepsilon-2 \tau u w} \exp \left(-\tau u \int_{w}^{1} \frac{(u v)^{2}-1}{\varepsilon-2 \tau u v} d v\right) d w
$$

und daraus

$$
\begin{gathered}
\left|\operatorname{Rg}_{K}(\tau, \varepsilon u)\right| \\
\leq \tau^{K+1}|u| \int_{0}^{1} \frac{\left|P_{K+1}^{\prime}(\varepsilon u w)\right|}{|\varepsilon-2 \tau u w|} \exp \left(-\Re\left[\tau u \int_{w}^{1} \frac{(u v)^{2}-1}{\varepsilon-2 \tau u v} d v\right]\right) d w .
\end{gathered}
$$

Mit der Abkürzung

$$
x:=2 \sqrt{2} \tau u
$$


ist

$$
|\varepsilon-2 \tau u w|=\frac{1}{\sqrt{2}}|1+i-x w|=\frac{1}{\sqrt{2}}\left[1+(x w-1)^{2}\right]^{\frac{1}{2}}
$$

und

$$
\begin{gathered}
-\Re\left[\tau u \int_{w}^{1} \frac{(u v)^{2}-1}{\varepsilon-2 \tau u v} d v\right] \\
=-\Re\left[\frac{x}{2} \int_{w}^{1} \frac{(u v)^{2}-1}{1+i-x v} d v\right]=\frac{x}{2} \int_{w}^{1} \frac{\left(u^{2} v^{2}-1\right)(x v-1)}{1+(x v-1)^{2}} d v \\
=\frac{x}{2} u^{2} \int_{w}^{1} v^{2} \frac{x v-1}{1+(x v-1)^{2}} d v-\frac{x}{2} \int_{w}^{1} \frac{x v-1}{1+(x v-1)^{2}} d v .
\end{gathered}
$$

Für $x \neq 0$ können wir im ersten Integral $v$ mit $v / x$ substituieren. Das zweite Integral ist elementar lösbar - nach Multiplikation mit $2 x$ wird der Zähler des Integranden gleich der Ableitung des Nenners - und wir erhalten

$$
\begin{gathered}
-\Re\left[\tau u \int_{w}^{1} \frac{(u v)^{2}-1}{\varepsilon-2 \tau u v} d v\right] \\
=\frac{u^{2}}{2 x^{2}} \int_{x w}^{x} v^{2} \frac{v-1}{1+(v-1)^{2}} d v-\frac{1}{4} \log \left[1+(x-1)^{2}\right]+\frac{1}{4} \log \left[1+(x w-1)^{2}\right] .
\end{gathered}
$$

Setzen wir noch

$$
\varphi(x, w):=\int_{x w}^{x} v^{2} \frac{v-1}{1+(v-1)^{2}} d v
$$

dann wird mit 3.13

$$
\begin{gathered}
\frac{1}{|\varepsilon-2 \tau u w|} \exp \left(-\Re\left[\tau u \int_{w}^{1} \frac{(u v)^{2}-1}{\varepsilon-2 \tau u v} d v\right]\right) \\
=\sqrt{2}\left[1+(x w-1)^{2}\right]^{-\frac{1}{4}}\left[1+(x-1)^{2}\right]^{-\frac{1}{4}} \exp \left(\frac{u^{2}}{2 x^{2}} \varphi(x, w)\right) .
\end{gathered}
$$

Die Einschränkung $x \neq 0$ kann wegen der aus (3.12) und (3.14) folgenden Beziehung

$$
\lim _{x \rightarrow 0} \frac{u^{2}}{2 x^{2}} \varphi(x, w)=\frac{1}{16 \tau^{2}} \varphi(0, w)=0
$$

natürlich jetzt fortfallen. Aus (3.11) erhalten wir daher, wenn wir die für $0<w<1$ geltende Abschätzung

$$
\left[1+(x w-1)^{2}\right]^{-\frac{1}{4}}\left[1+(x-1)^{2}\right]^{-\frac{1}{4}}<\left\{\begin{array}{cc}
\frac{1}{\sqrt{2}} & \text { für } x<0 \\
1 & \text { für } x \geq 0
\end{array}\right.
$$


berücksichtigen,

$$
\left|\operatorname{Rg}_{K}(\tau, \varepsilon u)\right|<\left\{\begin{array}{cl}
\tau^{K+1}|u| \int_{0}^{1} e^{u^{2} \varphi(x, w) / 2 x^{2}}\left|P_{K+1}^{\prime}(\varepsilon u w)\right| d w & \text { für } x<0, \\
\tau^{K+1} \sqrt{2}|u| \int_{0}^{1} e^{u^{2} \varphi(x, w) / 2 x^{2}}\left|P_{K+1}^{\prime}(\varepsilon u w)\right| d w & \text { für } x \geq 0 .
\end{array}\right.
$$

Eine von $w$ unabhängige Abschätzung für $\varphi(x, w)$ läßt sich wie folgt gewinnen. Bezeichnen wir der Einfachheit halber die partielle Ableitung von $\varphi(x, w)$ nach $w$ $\operatorname{mir} \varphi^{\prime}(x, w)$, dann wird wegen 3.14

$$
\varphi^{\prime}(x, w)=-x^{3} w^{2} \frac{x w-1}{1+(x w-1)^{2}} .
$$

Hieraus ergibt sich

$$
\varphi^{\prime}(x, w) \begin{cases}<0 & \text { für } 0<w<1 \text { und } x<0, \\ >0 & \text { für } 0<w<1 \text { und } 0<x \leq 1, \\ >0 & \text { für } 0<w<\frac{1}{x} \text { und } x>1, \\ =0 & \text { für } w=\frac{1}{x} \text { und } x>1, \\ <0 & \text { für } \frac{1}{x}<w<1 \text { und } x>1 .\end{cases}
$$

Als Funktion von $w$ ist $\varphi(x, w)$ daher in dem Bereich $0<w<1$ für $x<0$ streng monoton fallend, für $0<x \leq 1$ streng monoton steigend und hat für $x>1$ bei $w=1 / x$ ein lokales Maximum, das wegen der strengen Monotonie von $\varphi(x, w)$, die aus den letzten drei Abschätzungen von $\varphi^{\prime}(x, w)$ folgt, gleichzeitig das Maximum von $\varphi(x, w)$ in dem gesamten Bereich $0 \leq w \leq 1$ ist. Folglich können wir $\varphi(x, w)$ für $0 \leq w \leq 1$ mit

$$
\varphi(x, w) \leq \begin{cases}\varphi(x, 0) & \text { für } x<0, \\ \varphi(x, 1)=0 & \text { für } 0 \leq x \leq 1 \\ \varphi\left(x, \frac{1}{x}\right) & \text { für } x>1\end{cases}
$$

abschätzen, wobei sich die Gültigkeit dieser Abschätzung für $x=0$ aus 3.15 ergibt. Setzen wir $f(x):=\varphi(x, 0)$, so ist nach 3.14

$$
\begin{aligned}
f(x) & =\int_{0}^{x} v^{2} \frac{v-1}{1+(v-1)^{2}} d v \\
\varphi\left(x, \frac{1}{x}\right) & =\int_{1}^{x} v^{2} \frac{v-1}{1+(v-1)^{2}} d v=f(x)-f(1),
\end{aligned}
$$


und wir erhalten aus 3.16

$$
\left|\operatorname{Rg}_{K}(\tau, \varepsilon u)\right|< \begin{cases}\tau^{K+1} e^{u^{2} f(x) / 2 x^{2}}|u| \int_{0}^{1}\left|P_{K+1}^{\prime}(\varepsilon u w)\right| d w & \text { für } x<0, \\ \tau^{K+1} \sqrt{2}|u| \int_{0}^{1}\left|P_{K+1}^{\prime}(\varepsilon u w)\right| d w & \text { für } 0 \leq x \leq 1, \\ \tau^{K+1} \sqrt{2} e^{u^{2}[f(x)-f(1)] / 2 x^{2}} & \\ \times|u| \int_{0}^{1}\left|P_{K+1}^{\prime}(\varepsilon u w)\right| d w & \text { für } x>1 .\end{cases}
$$

Zur weiteren Abschätzung verwenden wir die Darstellung 1.22 der Polynome $P_{n}(z)$ und erhalten

$$
\begin{aligned}
|u| \int_{0}^{1}\left|P_{K+1}^{\prime}(\varepsilon u w)\right| d w & =|u| \int_{0}^{1}\left|\sum_{k=0}^{K+1} i^{k-K-1} \frac{a_{k}^{(K+1)}}{(K+2 k) !}(\varepsilon u w)^{K+2 k}\right| d w \\
& \leq|u| \sum_{k=0}^{K+1} \frac{a_{k}^{(K+1)}}{(K+2 k) !}|u|^{K+2 k} \int_{0}^{1} w^{K+2 k} d w \\
& =\sum_{k=0}^{K+1} \frac{a_{k}^{(K+1)}}{(K+1+2 k) !}|u|^{K+1+2 k} .
\end{aligned}
$$

Aus (3.17) folgt hiermit, wenn wir den letzten Ausdruck entsprechend (3.9) mit $V_{K}(u)$ bezeichnen und die Abschätzungen für $f(x)$ bzw. $f(x)-f(1)$ aus Satz 4.4.2. S. 81 verwenden

$$
\left|\operatorname{Rg}_{K}(\tau, \varepsilon u)\right|<\left\{\begin{aligned}
e^{u^{2} / 4} V_{K}(u) \tau^{K+1} & \text { für } x \leq-\theta, \\
e^{\omega u^{2} / 4} V_{K}(u) \tau^{K+1} & \text { für }-\theta<x<0, \\
\sqrt{2} V_{K}(u) \tau^{K+1} & \text { für } 0 \leq x \leq 1, \\
\sqrt{2} e^{0.28 u^{2}} V_{K}(u) \tau^{K+1} & \text { für } x>1 .
\end{aligned}\right.
$$

Dabei ist $\theta>0$ und $\omega$ durch 3.10 gegeben. Wegen 3.12 ist das gerade die Behauptung des Satzes.

Mit Hilfe dieses Satzes läßt sich nun leicht eine Abschätzung für $\operatorname{RS}_{K}(\tau)$ gewinnen. Wir verwenden zur Vereinfachung der Schreibweise neben der Variablen $\tau$ jetzt auch wieder die Variable $t$ - bekanntlich ist $\tau=1 /(2 \sqrt{2 t})$ bzw. $t=1 /\left(8 \tau^{2}\right)-$ und haben den

Satz 3.1.3. Es sei $t_{0}>(3 K+4) / 0.44$ und $\tau_{0}:=1 /\left(2 \sqrt{2 t_{0}}\right)$. Dann ist für $t \geq t_{0}$ bzw. $\tau \leq \tau_{0}$

$$
\left|\operatorname{RS}_{K}(\tau)\right|<\left(Y_{K}^{(1)}+Y_{K}^{(2)}+Y_{K}^{(3)}+Y_{K}^{(4)}\right) \tau^{K+1}
$$


mit

$$
\begin{aligned}
Y_{K}^{(1)} & :=\frac{\left(\theta \sqrt{t_{0}}\right)^{K} e^{-\theta^{2} t_{0} / 4}}{\sqrt{\pi} \sinh \frac{\theta}{4} \sqrt{2 \pi t_{0}}} \sum_{k=0}^{K+1} \frac{\left(\theta^{2} t_{0}\right)^{k} a_{k}^{(K+1)}}{(K+1+2 k) !}\left(\frac{K}{2}+k+1\right), \\
Y_{K}^{(2)} & :=\frac{1}{\sqrt{2} \pi(\sqrt{w})^{K+1}} \sum_{k=0}^{K+1} \frac{w^{-k} a_{k}^{(K+1)}}{(K+1+2 k) !} \Gamma\left(\frac{K+1}{2}+k\right), \\
Y_{K}^{(3)} & :=\frac{(\sqrt{2})^{K+1}}{\pi} \sum_{k=0}^{K+1} \frac{2^{k} a_{k}^{(K+1)}}{(K+1+2 k) !} \Gamma\left(\frac{K+1}{2}+k\right), \\
Y_{K}^{(4)} & :=\frac{\left(\sqrt{t_{0}}\right)^{K} e^{-0.22 t_{0}}}{0.44 \sqrt{2 \pi} \sinh \frac{\sqrt{2 \pi t_{0}}}{4}} \sum_{k=0}^{K+1} \frac{t_{0}^{k} a_{k}^{(K+1)}}{(K+1+2 k) !}\left(\frac{K}{2}+k+1\right) .
\end{aligned}
$$

Dabei ist $\theta$ eine beliebige reelle Zahl, die nur der Bedingung $\theta>\sqrt{2(3 K+4) / t_{0}}$ genügen muß und

$$
w:=\frac{1}{4}+\frac{1}{2 \theta}-\frac{1}{\theta^{2}} \arctan \frac{\theta}{\theta+2} .
$$

Diese Abschätzung von $\operatorname{RS}_{K}(\tau)$ gilt gleichmäßig in $q$.

Beweis. Für reelle $x$ und $y$ ist

$$
|\cosh (x+i y)|^{2}=\sinh ^{2} x+\cos ^{2} y .
$$

Daher ist für reelle $u$

$$
\begin{aligned}
\left|\cosh \frac{\sqrt{\pi}}{2}(i q+\varepsilon u)\right| & =\left|\cosh \frac{\sqrt{2 \pi}}{4}[u+i(\sqrt{2} q+u)]\right| \\
& =\left[\sinh ^{2} \frac{\sqrt{2 \pi}}{4} u+\cos ^{2} \frac{\sqrt{2 \pi}}{4}(\sqrt{2} q+u)\right]^{\frac{1}{2}} \\
& \geq\left|\sinh \frac{\sqrt{2 \pi}}{4} u\right|=\sinh \frac{\sqrt{2 \pi}}{4}|u|
\end{aligned}
$$

und aus Satz 3.1 .1 folgt

$$
\left|\operatorname{RS}_{K}(\tau)\right| \leq \frac{1}{2 \sqrt{\pi}} \int_{-\infty}^{+\infty} \frac{e^{-u^{2} / 2}}{\sinh \frac{\sqrt{2 \pi}}{4}|u|}\left|\operatorname{Rg}_{K}(\tau, \varepsilon u)\right| d u
$$

gleichmäßig für die von uns betrachteten $q$ mit $|q| \leq \sqrt{\pi}$, denn diese Abschätzung ist von $q$ unabhängig. Wir zerlegen das Integral entsprechend den Geltungsbereichen der Abschätzungen von $\left|\operatorname{Rg}_{K}(\tau, \varepsilon u)\right|$ aus dem vorigen Satz und haben wegen $1 /(2 \sqrt{2} \tau)=\sqrt{t}$

$$
\left|\mathrm{RS}_{K}(\tau)\right| \leq J_{1}+J_{2}+J_{3}+J_{4}
$$

mit

$$
J_{1}:=\int_{-\infty}^{-\theta \sqrt{t}} \Psi_{K}(\tau, u) d u, \quad J_{2}:=\int_{-\theta \sqrt{t}}^{0} \Psi_{K}(\tau, u) d u
$$




$$
J_{3}:=\int_{0}^{\sqrt{t}} \Psi_{K}(\tau, u) d u, \quad J_{4}:=\int_{\sqrt{t}}^{\infty} \Psi_{K}(\tau, u) d u
$$

und der Abkürzung

$$
\Psi_{K}(\tau, u):=\frac{1}{2 \sqrt{\pi}} \cdot \frac{e^{-u^{2} / 2}}{\sinh \frac{\sqrt{2 \pi}}{4}|u|}\left|\operatorname{Rg}_{K}(\tau, \varepsilon u)\right|
$$

Für reelle $a$ und positive reelle $x$ bezeichnen wir die unvollständige Gammafunktion 3 )

$$
\int_{x}^{\infty} e^{-v} v^{a-1} d v
$$

wie üblich mit $\Gamma(a, x)$ und schätzen die Ausdrücke $J_{1}$ bis $J_{4}$ mit Hilfe von Satz 3.1 .2 ab. Dabei sei $t_{0}>(3 K+4) / 0.44$ und $t \geq t_{0}$.

a) Abschätzung von $J_{1}$

$$
\begin{aligned}
J_{1} & <\frac{\tau^{K+1}}{2 \sqrt{\pi}} \int_{\theta \sqrt{t}}^{\infty} \frac{e^{-u^{2} / 2}}{\sinh \frac{\sqrt{2 \pi}}{4} u} e^{u^{2} / 4} V_{K}(u) d u \\
& \leq \frac{\tau^{K+1}}{2 \sqrt{\pi} \sinh \frac{\theta}{4} \sqrt{2 \pi t_{0}}} \int_{\theta \sqrt{t_{0}}}^{\infty} e^{-u^{2} / 4} \sum_{k=0}^{K+1} \frac{a_{k}^{(K+1)}}{(K+1+2 k) !} u^{K+1+2 k} d u .
\end{aligned}
$$

Mit der Substitution $u=2 \sqrt{v}$ folgt daraus

$$
J_{1}<\frac{\tau^{K+1}}{\sqrt{\pi} \sinh \frac{\theta}{4} \sqrt{2 \pi t_{0}}} \sum_{k=0}^{K+1} \frac{2^{K+2 k} a_{k}^{(K+1)}}{(K+1+2 k) !} \Gamma\left(\frac{K}{2}+k+1, \frac{\theta^{2} t_{0}}{4}\right) .
$$

Für $\theta^{2} t_{0} / 4>K / 2+k+1$ ist die Abschätzung aus Satz 4.4.3. S. 84 anwendbar. Wegen $K / 2+k+1 \leq(3 K+4) / 2$ wird damit

$$
J_{1}<\left[\frac{\left(\theta \sqrt{t_{0}}\right)^{K} e^{-\theta^{2} t_{0} / 4}}{\sqrt{\pi} \sinh \frac{\theta}{4} \sqrt{2 \pi t_{0}}} \sum_{k=0}^{K+1} \frac{\left(\theta^{2} t_{0}\right)^{k} a_{k}^{(K+1)}}{(K+1+2 k) !}\left(\frac{K}{2}+k+1\right)\right] \tau^{K+1},
$$

falls $\theta$ der Bedingung $\theta>\sqrt{2(3 K+4) / t_{0}}$ genügt.

b) Abschätzung von $J_{2}$

$$
J_{2}<\frac{\tau^{K+1}}{2 \sqrt{\pi}} \int_{0}^{\theta \sqrt{t}} e^{-u^{2} / 2} \frac{u}{\sinh \frac{\sqrt{2 \pi}}{4} u} e^{\omega u^{2} / 4} \frac{V_{K}(u)}{u} d u .
$$

\footnotetext{
3) Siehe [1. Kap. 6.5].
} 
Wegen $u / \sinh u \leq 1$ für reelle $u$ ist

$$
\frac{u}{\sinh \frac{\sqrt{2 \pi}}{4} u} \leq \frac{4}{\sqrt{2 \pi}}
$$

Damit wird

$$
J_{2}<\frac{\sqrt{2} \tau^{K+1}}{\pi} \int_{0}^{\infty} e^{-(2-\omega) u^{2} / 4} \sum_{k=0}^{K+1} \frac{a_{k}^{(K+1)}}{(K+1+2 k) !} u^{K+2 k} d u .
$$

Wir setzen

$$
w:=\frac{2-\omega}{4} .
$$

Wegen $\omega<1$ ist dann $w>1 / 4>0$ und mit der Substitution $u=\sqrt{v / w}$ folgt

$$
J_{2}<\left[\frac{1}{\sqrt{2} \pi(\sqrt{w})^{K+1}} \sum_{k=0}^{K+1} \frac{w^{-k} a_{k}^{(K+1)}}{(K+1+2 k) !} \Gamma\left(\frac{K+1}{2}+k\right)\right] \tau^{K+1} .
$$

Die in 3.18 angegebene Darstellung von $w$ ergibt sich aus der Definition von $\omega$ in (3.10.

c) Abschätzung von $J_{3}$

$$
J_{3}<\frac{\tau^{K+1}}{2 \sqrt{\pi}} \int_{0}^{\sqrt{t}} e^{-u^{2} / 2} \frac{u}{\sinh \frac{\sqrt{2 \pi}}{4} u} \sqrt{2} \frac{V_{K}(u)}{u} d u .
$$

Mit (3.21) erhalten wir

$$
J_{3}<\frac{2 \tau^{K+1}}{\pi} \int_{0}^{\infty} e^{-u^{2} / 2} \sum_{k=0}^{K+1} \frac{a_{k}^{(K+1)}}{(K+1+2 k) !} u^{K+2 k} d u
$$

und daraus mit der Substitution $u=\sqrt{2 v}$

$$
J_{3}<\left[\frac{(\sqrt{2})^{K+1}}{\pi} \sum_{k=0}^{K+1} \frac{2^{k} a_{k}^{(K+1)}}{(K+1+2 k) !} \Gamma\left(\frac{K+1}{2}+k\right)\right] \tau^{K+1} .
$$

d) Abschätzung von $J_{4}$

$$
\begin{aligned}
J_{4} & <\frac{\tau^{K+1}}{2 \sqrt{\pi}} \int_{\sqrt{t}}^{\infty} \frac{e^{-u^{2} / 2}}{\sinh \frac{\sqrt{2 \pi}}{4} u} \sqrt{2} e^{0.28 u^{2}} V_{K}(u) d u \\
& \leq \frac{\tau^{K+1}}{\sqrt{2 \pi} \sinh \frac{\sqrt{2 \pi t_{0}}}{4}} \int_{\sqrt{t_{0}}}^{\infty} e^{-0.22 u^{2}} \sum_{k=0}^{K+1} \frac{a_{k}^{(K+1)}}{(K+1+2 k) !} u^{K+1+2 k} d u .
\end{aligned}
$$


Wir substituieren mit $u=\sqrt{v / 0.22}$ und erhalten

$$
J_{4}<\frac{\tau^{K+1}}{2 \sqrt{2 \pi} \sinh \frac{\sqrt{2 \pi t_{0}}}{4}} \sum_{k=0}^{K+1} \frac{0.22^{-\frac{K}{2}-k-1} a_{k}^{(K+1)}}{(K+1+2 k) !} \Gamma\left(\frac{K}{2}+k+1,0.22 t_{0}\right) .
$$

Da wir $0.22 t_{0}>(3 K+4) / 2$ vorausgesetzt haben, gilt für alle $k$ mit $0 \leq k \leq K+1$ die Beziehung $0.22 t_{0}>K / 2+k+1$, so daß wir wieder die Abschätzung aus Satz 4.4.3. S. 84 verwenden können. Damit wird

$$
J_{4}<\left[\frac{\left(\sqrt{t_{0}}\right)^{K} e^{-0.22 t_{0}}}{0.44 \sqrt{2 \pi} \sinh \frac{\sqrt{2 \pi t_{0}}}{4}} \sum_{k=0}^{K+1} \frac{t_{0}^{k} a_{k}^{(K+1)}}{(K+1+2 k) !}\left(\frac{K}{2}+k+1\right)\right] \tau^{K+1} .
$$

Bezeichnen wir die in den eckigen Klammern stehenden Ausdrücke in den Abschätzungen 3.20 - 3.24 der Reihe nach mit $Y_{K}^{(1)}$ bis $Y_{K}^{(4)}$, so ist das wegen 3.19 gerade die Behauptung des Satzes.

Bei geeigneter Vorgabe von $t_{0}$ und $\theta$, also unter Berücksichtigung der Nebenbedingungen $t_{0}>(3 K+4) / 0.44$ und $\theta>\sqrt{2(3 K+4) / t_{0}}$, werden die $Y_{K}^{(l)}(1 \leq l \leq 4)$ für jedes $K \geq 0$ konstante Größen. Folglich ist für hinreichend kleine $\tau$

$$
\left|\mathrm{RS}_{K}(\tau)\right|<c \tau^{K+1}
$$

mit einer nur von $K$, aber nicht von $\tau$ und $q$ abhängigen Konstanten $c$, und wir haben wegen 3.2 den

Satz 3.1.4. Für $K \geq 0$ ist gleichmäßig in $q$

$$
S=\sum_{n=0}^{K} B_{n}(q) \tau^{n}+O\left(\tau^{K+1}\right) \quad \text { für } \tau \rightarrow 0 .
$$

Die formale Potenzreihe

$$
\sum_{n=0}^{\infty} B_{n}(q) \tau^{n}
$$

ist die asymptotische Entwicklung des Ausdruckes $S$ für festes $|q| \leq \sqrt{\pi}$ und $\tau \rightarrow 0$ bzw. $t \rightarrow+\infty$.

Die Ausdrücke $Y_{k}^{(l)}(1 \leq l \leq 4)$ in Satz 3.1 .3 mögen dem Leser etwas kompliziert erscheinen. Es ist aber wenig sinnvoll, sie durch eine weitere Abschätzung zu vereinfachen, denn dazu wären Abschätzungen für die Zahlen $a_{k}^{(n)}$ notwendig, die nur bei entsprechend hohem Aufwand durch Untersuchung ihrer Rekursionsformel 1.23, S. 16 bzw. des Wachstums der Polynome $P_{n}(z)$ in

$$
g(\tau, z)=\sum_{n=0}^{\infty} P_{n}(z) \tau^{n} \quad(2 \tau|z|<1)
$$

zu gewinnen wären. Unter der Voraussetzung, daß die $a_{k}^{(K+1)}$ exakt bekannt sind, kann man sich diese mühevollen Abschätzungen ersparen. Die direkte Berechnung $\operatorname{der} Y_{K}^{(l)}(1 \leq l \leq 4)$ aus den in Satz 3.1 .3 angegebenen Darstellungen bereitet dann nämlich keine Schwierigkeiten. Auf diese Weise kann man recht einfach explizite Abschätzungen für $\left|\operatorname{RS}_{K}(\tau)\right|$ herleiten, wie der folgende Satz für den Fall $K=10$ zeigt. 
Satz 3.1.5. Für $\tau \leq 1 / 40$ bzw. $t \geq 200$ ist gleichmäßig in $q$

$$
\left|\mathrm{RS}_{10}(\tau)\right|<1.4 \cdot 10^{9} \tau^{11} .
$$

Beweis. Wir setzen in Satz $3.1 .3 K=10$ und $t_{0}=200$. Dann wird $\tau_{0}=1 / 40$ und die Bedingung $t_{0}>(3 K+4) / 0.44=34 / 0.44$ ist erfüllt. Mit

$$
\Gamma\left(\frac{11}{2}+k\right)=\frac{(2 k+10) !}{2^{2 k+10}(k+5) !} \sqrt{\pi}
$$

folgt nach einfachen Umformungen

$$
\begin{aligned}
& Y_{10}^{(1)}=\frac{\left(200 \theta^{2}\right)^{5} e^{-50 \theta^{2}}}{\sqrt{\pi} \sinh (5 \sqrt{\pi} \theta)} \sum_{k=0}^{11} \frac{\left(200 \theta^{2}\right)^{k} a_{k}^{(11)}}{(2 k+11) !}(k+6), \\
& Y_{10}^{(2)}=\frac{\sqrt{2}}{\sqrt{\pi}(\sqrt{4 w})^{11}} \sum_{k=0}^{11} \frac{(4 w)^{-k} a_{k}^{(11)}}{(2 k+11)(k+5) !}, \\
& Y_{10}^{(3)}=\frac{1}{\sqrt{\pi}(\sqrt{2})^{9}} \sum_{k=0}^{11} \frac{2^{-k} a_{k}^{(11)}}{(2 k+11)(k+5) !}, \\
& Y_{10}^{(4)}=\frac{200^{5} e^{-44}}{0.44 \sqrt{2 \pi} \sinh (5 \sqrt{\pi})} \sum_{k=0}^{11} \frac{200^{k} a_{k}^{(11)}}{(2 k+11) !}(k+6) .
\end{aligned}
$$

Den abgesehen von der Nebenbedingung

$$
\theta>\sqrt{\frac{2}{t_{0}}(3 K+4)}=\frac{\sqrt{34}}{10}
$$

noch frei wählbaren Parameter $\theta$ bestimmen wir so, daß die Summe $Y_{10}^{(1)}+Y_{10}^{(2)}$ möglichst klein wird. Ein recht guter Wert ist

$$
\theta=\frac{3}{4}>\frac{\sqrt{34}}{10}=0.58309 \ldots
$$

Damit wird

$$
w=\frac{11}{12}-\frac{16}{9} \arctan \frac{3}{11}=0.443329690398 \ldots
$$

und mit den Zahlen $a_{k}^{(11)}$ aus Tabelle I, S. 91 berechnet man

$$
\begin{array}{ll}
Y_{10}^{(1)}=4.07738 \ldots \cdot 10^{7}<4.08 \cdot 10^{7}, & Y_{10}^{(2)}=9.67772 \ldots \cdot 10^{8}<9.68 \cdot 10^{8}, \\
Y_{10}^{(3)}=3.90019 \ldots \cdot 10^{8}<3.91 \cdot 10^{8}, & Y_{10}^{(4)}=2551.10 \ldots<10^{4} .
\end{array}
$$

Folglich ist für $\tau \leq 1 / 40$ bzw. $t \geq 200$ gleichmäßig in $q$

$$
\begin{aligned}
\left|\mathrm{RS}_{10}(\tau)\right| & <\left(4.08 \cdot 10^{7}+9.68 \cdot 10^{8}+3.91 \cdot 10^{8}+10^{4}\right) \tau^{11} \\
& =1.39981 \cdot 10^{9} \tau^{11} \\
& <1.4 \cdot 10^{9} \tau^{11}
\end{aligned}
$$

wie behauptet. 
Mit Hilfe von Tabelle I kann man auf demselben Wege auch für $K<10 \mathrm{zu}$ expliziten Abschätzungen von $\left|\mathrm{RS}_{K}(\tau)\right|$ gelangen. Im nächsten Abschnitt benötigen wir jedoch nur die eben bewiesene Abschätzung von $\left|\mathrm{RS}_{10}(\tau)\right|$, so daß wir auf die Durchführung dieser Abschätzungen verzichten können.

Abschließend sei noch darauf hingewiesen, daß die Abschätzung von $\left|\operatorname{RS}_{K}(\tau)\right|$ in Satz 3.1.3 nur durch Entwicklung der Funktion $g(\tau, z)$ in eine Potenzreihe nach $\tau$ auf so einfache Weise zu gewinnen war. Hätten wir die andere Möglichkeit gewählt und $g(\tau, z)$ in eine Potenzreihe nach $z$ entwickelt, so wären die Koeffizienten dieser Reihe Polynome in $\tau$ geworden, was bei einer Satz 3.1 .3 entsprechenden Restabschätzung wegen der dann fehlenden Anordnung nach Potenzen von $\tau$ zu fast unüberwindlichen Schwierigkeiten geführt hätte. Der Leser vergleiche dazu noch einmal die Fußnote 8) auf S. 14.

\subsection{Explizite Abschätzung der ersten 11 Restglieder}

Wir betrachten zunächst die in Satz 1.3.1. S. 18 angegebene Darstellung der Riemann-Siegel-Formel. Wenn wir dort die formale Reihe $\sum_{n=0}^{\infty} \widehat{C}_{n}(q) \tau^{n}$ nach dem K-ten Gliede $(K \geq 0)$ abbrechen, erhalten wir einen Ausdruck, der für alle $K \geq 0$ sinnvoll ist und es stellt sich die Frage, mit welcher Genauigkeit dieser Ausdruck die Funktion $Z(t)$ approximiert. Dazu führen wir die Restglieder

$$
R_{K}(t):=Z(t)-2 \sum_{n=1}^{N} \frac{\cos (\vartheta(t)-t \log n)}{\sqrt{n}}-\frac{(-1)^{N-1}}{\sqrt{a}} \sum_{n=0}^{K} \widehat{C}_{n}(q) \tau^{n}
$$

mit

$$
a:=\sqrt{\frac{t}{2 \pi}}, \quad N:=\lfloor a\rfloor, \quad q:=\sqrt{\pi}[1-2(a-N)], \quad \tau:=\frac{1}{2 \sqrt{2 t}}
$$

ein, die dann im weiteren näher untersucht werden müssen und vereinbaren, daß bis zum Ende dieses Abschnittes $K$ ganzzahlig $\geq 0$ und $t$ reell $>0$ zu nehmen ist.

Eine zweite, zur Abschätzung besonders gut geeignete Darstellung von $R_{K}(t)$ ergibt sich, wenn man (3.25) auf die Lehmersche Form (Satz 2.1.6, S. 30] transformiert:

$$
R_{K}(t)=Z(t)-2 \sum_{n=1}^{N} \frac{\cos (\vartheta(t)-t \log n)}{\sqrt{n}}-\frac{(-1)^{N-1}}{\sqrt{a}} \sum_{n=0}^{K} \frac{C_{n}(z)}{a^{n}}
$$

mit $a$ und $N$ wie oben und $z:=1-2(a-N)$.

Das asymptotische Verhalten von $R_{K}(t)$ für $t \rightarrow+\infty$ läßt sich mit den Ergebnissen des letzten Abschnittes sofort angeben:

Satz 3.2.1. Für $t \rightarrow+\infty$ ist

$$
R_{K}(t)=O\left(t^{-(2 K+3) / 4}\right) .
$$

Die Riemann-Siegel-Formel stellt $Z(t)$ für $t \rightarrow+\infty$ asymptotisch dar. 
Beweis. Aus den Sätzen 3.1.4 und 4.2.4 (c), S. 74 folgt die asymptotische Entwicklung

$$
U \cdot S \sim \sum_{n=0}^{\infty} \widetilde{C}_{n}(q) \tau^{n} \quad(\tau \rightarrow 0,|q| \leq \sqrt{\pi}),
$$

deren Koeffizienten sich nach den Formeln 1.30 errechnen, und es ist gleichmäßig in $q$

$$
U \cdot S=\sum_{n=0}^{K} \widetilde{C}_{n}(q) \tau^{n}+O\left(\tau^{K+1}\right) \quad \text { für } \tau \rightarrow 0 .
$$

Durch Übergang zum Realteil folgt dann - ebenfalls gleichmäßig in $q-$

$$
\Re(U \cdot S)=\sum_{n=0}^{K} \widehat{C}_{n}(q) \tau^{n}+O\left(\tau^{K+1}\right) \quad \text { für } \tau \rightarrow 0
$$

und daraus, wenn wir die Darstellung von $Z(t)$ aus Satz 1.2.1. S. 12 mit 3.25 vergleichen und beachten, daß $q$ dort immer der Bedingung $-\sqrt{\pi}<q \leq \sqrt{\pi}$ genügt,

$$
R_{K}(t)=t^{-\frac{1}{4}} O\left(\tau^{K+1}\right)=t^{-\frac{1}{4}} O\left(t^{-(K+1) / 2}\right)=O\left(t^{-(2 K+3) / 4}\right) \quad \text { für } t \rightarrow+\infty .
$$

Daher ist die Riemann-Siegel-Formel eine asymptotische Entwicklung der Funktion $Z(t)$ für $t \rightarrow+\infty$ und der Satz ist bewiesen.

Für jedes $K$ und hinreichend große $t$ gibt es also eine Abschätzung der Form

$$
\left|R_{K}(t)\right|<c(K) t^{-(2 K+3) / 4}
$$

mit positiven Konstanten $c(K)$, die nur von $K$ aber nicht von $t$ abhängen.

Damit können wir jetzt die Unstetigkeitsstellen der Riemann-Siegel-Formel untersuchen. An den Stellen $t=t_{M}=2 \pi M^{2}$ ( $M$ ganz $\left.>0\right)$, an denen $N$ von $M-1$ auf $M$ springt, ist $R_{K}(t)$ nämlich nicht stetig, wie man leicht nachweist, wenn man in (3.25) oder (3.26) den links- bzw. rechtsseitigen Grenzübergang $t \rightarrow t_{M}\left(t<t_{M}\right)$ und $t \rightarrow t_{M}\left(t>t_{M}\right)$ vornimmt. Daher sind die auf den rechten Seiten von 3.25 bzw. (3.26) stehenden Ausdrücke, mit denen wir die stetige - sogar analytische Funktion $Z(t)$ approximieren wollen, an den Stellen $t_{M}$ ebenfalls unstetig. Die Verwendbarkeit dieser Ausdrücke zur numerischen Berechnung von $Z(t)$ wird davon für große $t$ aber nicht negativ beeinflußt; denn nach 3.28 läßt sich die Höhe des Sprungs von $R_{K}(t)$ an den Stellen $t=t_{M}$ für große $M$ mit $2 c(K) t_{M}^{-(2 K+3) / 4}$ nach oben abschätzen. Für hinreichend große $M$ wird das - ebenso wie das Restglied $R_{K}(t)$ selbst - vernachlässigbar klein ${ }^{4)}$ so daß die Ausdrücke in 3.25 und 3.26 für große $t$ angenähert als stetige Funktionen betrachtet werden dürfen.

Für $K \leq 10$ geben wir jetzt explizite Abschätzungen für die Konstanten $c(K)$ an.

Satz 3.2.2. Für $t \geq 200$ und $0 \leq K \leq 10$ lassen sich die Restglieder $R_{K}(t)$

\footnotetext{
4) Hierauf beruht die Bestimmung der Werte $\widehat{C}_{2 n}(\sqrt{\pi})$ in Satz 2.1.5, S. 28 .
} 
(a) ohne Verwendung der Potenzreihenentwicklungen der Funktionen $C_{n}(z)$ mit

$$
\begin{aligned}
\left|R_{0}(t)\right|<0.47 t^{-3 / 4}, & \left|R_{1}(t)\right|<0.43 t^{-5 / 4}, & & \left|R_{6}(t)\right|<10.1 t^{-15 / 4}, \\
& \left|R_{2}(t)\right|<0.77 t^{-7 / 4}, & & \left|R_{7}(t)\right|<27.1 t^{-17 / 4}, \\
& \left|R_{3}(t)\right|<0.90 t^{-9 / 4}, & & \left|R_{8}(t)\right|<188 t^{-19 / 4}, \\
& \left|R_{4}(t)\right|<2.12 t^{-11 / 4}, & & \left|R_{9}(t)\right|<1934 t^{-21 / 4} \\
& \left|R_{5}(t)\right|<3.35 t^{-13 / 4}, & & \left|R_{10}(t)\right|<25966 t^{-23 / 4}
\end{aligned}
$$

grob abschätzen.

(b) Unter Verwendung dieser Potenzreihenentwicklungen ergeben sich für $K \leq 9$ die besseren Abschätzungen

$$
\begin{array}{rlrl}
\left|R_{0}(t)\right|<0.127 t^{-3 / 4}, & \left|R_{1}(t)\right|<0.053 t^{-5 / 4}, & & \left|R_{6}(t)\right|<0.661 t^{-15 / 4}, \\
& \left|R_{2}(t)\right|<0.011 t^{-7 / 4}, & & \left|R_{7}(t)\right|<9.2 t^{-17 / 4}, \\
& \left|R_{3}(t)\right|<0.031 t^{-9 / 4}, & & \left|R_{8}(t)\right|<130 t^{-19 / 4}, \\
& \left|R_{4}(t)\right|<0.017 t^{-11 / 4}, & & \left|R_{9}(t)\right|<1837 t^{-21 / 4}, \\
& \left|R_{5}(t)\right|<0.061 t^{-13 / 4} . &
\end{array}
$$

Für $K \leq 4$ sind diese Abschätzungen optimal.

Beweis. Es sei $t \geq 200$ bzw. $\tau \leq 1 / 40$. Mit $K=10$ folgt aus 3.2 und Satz 4.2 .4 (b), S. 74 unter Verwendung der Abkürzung

$$
u(\tau):=e^{-i\left(\frac{\tau^{2}}{6}+\frac{28}{45} \tau^{6}+\frac{3968}{315} \tau^{10}\right)}
$$

für $U \cdot S$ die Darstellung

$$
U \cdot S=u(\tau) \sum_{n=0}^{10} B_{n}(q) \tau^{n}+R^{(1)}
$$

mit

$$
R^{(1)}:=u(\tau) \cdot \operatorname{RS}_{10}(\tau)+\mathrm{RU}(\tau) \sum_{n=0}^{10} B_{n}(q) \tau^{n}+\mathrm{RU}(\tau) \cdot \operatorname{RS}_{10}(\tau) .
$$

Für $\tau \leq 1 / 40$ haben wir dann mit den Abschätzungen von $|\operatorname{RU}(\tau)|$ und $\left|\operatorname{RS}_{10}(\tau)\right|$ aus den Sätzen 4.2 .4 (b) und 3.1.5

$$
\begin{aligned}
\left|R^{(1)}\right| & <1.4 \cdot 10^{9} \tau^{11}+\frac{\tau^{11}}{101} \sum_{n=0}^{10}\left|B_{n}(q)\right| \tau^{n}+\frac{\tau^{11}}{101} \cdot 1.4 \cdot 10^{9} \tau^{11} \\
& \leq\left[1.4 \cdot 10^{9}+\frac{1}{101} \sum_{n=0}^{10} \frac{\left|B_{n}(q)\right|}{40^{n}}+\frac{1.4 \cdot 10^{9}}{101 \cdot 40^{11}}\right] \tau^{11} .
\end{aligned}
$$

Verwenden wir die Abschätzungen von $\left|B_{n}(q)\right|(0 \leq n \leq 10,|q| \leq \sqrt{\pi})$ aus Satz 4.4 .4 (b), S. 85, so ist gleichmäßig für diese $q$

$$
\sum_{n=0}^{10} \frac{\left|B_{n}(q)\right|}{40^{n}}<1.1
$$


Die Konstante in der obenstehenden eckigen Klammer läßt sich dann mit 1.41 $10^{9}$ nach oben abschätzen, so daß $\left|R^{(1)}\right|$ für $|q| \leq \sqrt{\pi}$ der Abschätzung

$$
\left|R^{(1)}\right|<1.41 \cdot 10^{9} \tau^{11}
$$

genügt. Daher ist $R^{(1)}=O\left(\tau^{11}\right)$ für $\tau \rightarrow 0$, und durch Vergleich von (3.27) - mit $K=10$ - und $(3.30)$ folgt, daß die Potenzreihe von $u(\tau) \sum_{n=0}^{10} B_{n}(q) \tau^{n}$ um den Punkt $\tau=0$ mindestens bis zur Potenz $\tau^{10}$ mit der formalen Reihe $\sum_{n=0}^{\infty} \widetilde{C}_{n}(q) \tau^{n}$ übereinstimmen muß. Betrachten wir für den Augenblick einmal $\tau$ als komplexe Veränderliche, dann ist $u(\tau) \sum_{n=0}^{10} B_{n}(q) \tau^{n}$ ( $q$ fest mit $|q| \leq \sqrt{\pi}$ ) eine ganze Funktion von $\tau$ und wir haben für alle komplexen $\tau$ die Identität

$$
u(\tau) \sum_{n=0}^{10} B_{n}(q) \tau^{n}=\sum_{n=0}^{10} \widetilde{C}_{n}(q) \tau^{n}+R^{(2)}
$$

mit

$$
R^{(2)}=\frac{1}{2 \pi i} \oint_{|x|=\varrho} \frac{u(x) \sum_{n=0}^{10} B_{n}(q) x^{n}}{x-\tau}\left(\frac{\tau}{x}\right)^{11} d x \quad(\varrho>|\tau|),
$$

wo auf dem Kreis mit dem Radius $\varrho$ einmal im mathematisch positiven Sinn um den Nullpunkt herum zu integrieren ist. Jetzt sei $\tau$ wieder reell mit $0<\tau \leq 1 / 40$. Wählen wir $\varrho>1 / 40$, so können wir $R^{(2)}$ für diese $\tau$ wie folgt abschätzen

$$
\left|R^{(2)}\right| \leq \frac{1}{2 \pi} \oint_{|x|=\varrho} \frac{|u(x)| \sum_{n=0}^{10}\left|B_{n}(q)\right| \varrho^{n}}{\varrho-\tau}\left(\frac{\tau}{\varrho}\right)^{11}|d x|,
$$

woraus sich mit 3.29 die Abschätzung

$$
\left|R^{(2)}\right| \leq\left[\frac{\exp \left(\frac{\varrho^{2}}{6}+\frac{28}{45} \varrho^{6}+\frac{3968}{315} \varrho^{10}\right)}{\left(\varrho-\frac{1}{40}\right) \varrho^{10}} \sum_{n=0}^{10}\left|B_{n}(q)\right| \varrho^{n}\right] \tau^{11}
$$

ergibt. Für $\left|B_{n}(q)\right|$ verwenden wir wie oben die expliziten Abschätzungen aus Satz 4.4.4 (b), S. 85. Der Ausdruck in der eckigen Klammer hängt dann nur noch von $\varrho$ ab und kann durch geeignete Wahl von $\varrho$ minimiert werden. Ein recht guter Wert dafür ist $\varrho=3 / 5>1 / 40$. Damit wird

$$
\sum_{n=0}^{10}\left|B_{n}(q)\right| \varrho^{n}<322318
$$

und

$$
\frac{\exp \left(\frac{\varrho^{2}}{6}+\frac{28}{45} \varrho^{6}+\frac{3968}{315} \varrho^{10}\right)}{\left(\varrho-\frac{1}{40}\right) \varrho^{10}}<340 .
$$

Folglich gilt für $\tau \leq 1 / 40$ die in $q$ gleichmäßige Abschätzung

$$
\left|R^{(2)}\right|<(340 \cdot 322318) \tau^{11}<1.1 \cdot 10^{8} \tau^{11} .
$$

\footnotetext{
5) Diese Darstellung des Restgliedes $R^{(2)}$ folgt in einfacher Weise aus den Cauchyschen Inte-
} gralformeln. Vgl. dazu [4. S. 135, Satz 24] Restglied einer Potenzreihe. 
Aus 3.30 und 3.32 erhalten wir jetzt

$$
U \cdot S=\sum_{n=0}^{10} \widetilde{C}_{n}(q) \tau^{n}+\operatorname{RUS}(\tau)
$$

mit

$$
\operatorname{RUS}(\tau):=R^{(1)}+R^{(2)}
$$

und nach 3.31 und 3.33 die Abschätzung

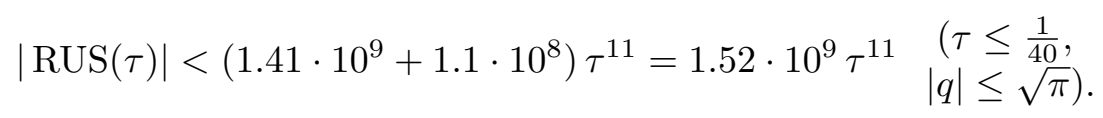

Gehen wir in (3.34) zum Realteil über

$$
\Re(U \cdot S)=\sum_{n=0}^{10} \widehat{C}_{n}(q) \tau^{n}+\Re[\operatorname{RUS}(\tau)]
$$

und setzen das in die Darstellung aus Satz 1.2.1. S. 12 von $Z(t)$ ein, so folgt durch Vergleich mit 3.25

$$
R_{10}(t)=(-1)^{N-1}\left(\frac{t}{2 \pi}\right)^{-\frac{1}{4}} \Re[\operatorname{RUS}(\tau)]
$$

Da $q$ in der Riemann-Siegel-Formel der Bedingung $|q| \leq \sqrt{\pi}$ genügt, ist die Abschätzung 3.35 anwendbar und wir erhalten, wenn wir zur Variablen $t$ zurückkehren und beachten, daß der Bereich $\tau \leq 1 / 40$ dem Bereich $t \geq 200$ entspricht, die für $t \geq 200$ gültige Abschätzung

$$
\left|R_{10}(t)\right| \leq\left(\frac{2 \pi}{t}\right)^{\frac{1}{4}}|\operatorname{RUS}(\tau)|<\left(\frac{2 \pi}{t}\right)^{\frac{1}{4}} \frac{1.52 \cdot 10^{9}}{(2 \sqrt{2 t})^{11}}<25966 t^{-23 / 4}
$$

wie in (a) dieses Satzes angegeben.

Die Abschätzungen von $R_{K}(t)$ für $K<10$ machen nun keine Schwierigkeiten mehr. In Gleichung (3.26) sei $0 \leq K \leq 9$. Subtrahieren wir davon dieselbe Gleichung mit $K=10$, so wird

$$
\begin{aligned}
R_{K}(t) & =\frac{(-1)^{N-1}}{\sqrt{a}} \sum_{n=K+1}^{10} \frac{C_{n}(z)}{a^{n}}+R_{10}(t) \\
& =(-1)^{N-1}\left(\frac{2 \pi}{t}\right)^{\frac{2 K+3}{4}} \sum_{n=K+1}^{10} C_{n}(z)\left(\frac{2 \pi}{t}\right)^{\frac{n-K-1}{2}}+R_{10}(t) .
\end{aligned}
$$

Es sei $t_{0} \geq 200$ fest vorgegeben. Für $t \geq t_{0}$ und $K \leq 9$ ist dann

$$
\left|R_{10}(t)\right|<25966 t^{-23 / 4}=25966 t^{\frac{K-10}{2}} t^{-\frac{2 K+3}{4}} \leq 25966 t^{\frac{K-10}{2}} t^{-\frac{2 K+3}{4}},
$$

und wir erhalten die Abschätzung

$$
\left|R_{K}(t)\right|<\left[(2 \pi)^{\frac{2 K+3}{4}} \sum_{n=K+1}^{10}\left|C_{n}(z)\right|\left(\frac{2 \pi}{t_{0}}\right)^{\frac{n-K-1}{2}}+25966 t^{\frac{K-10}{2}}\right] t^{-\frac{2 K+3}{4}},
$$


die für $0 \leq K \leq 9$ und $t \geq t_{0}$ gültig ist. Setzen wir hier $t_{0}=200$ und schätzen $\left|C_{n}(z)\right|$ mit Hilfe von Satz 2.3.4 (a), S. 38 ab ${ }^{6)}$ so lassen sich die Ausdrücke in der eckigen Klammer mit den in (a) dieses Satzes angegebenen Konstanten nach oben abschätzen. Auf dieselbe Weise ergeben sich die Abschätzungen (b), wenn man die aus den Potenzreihenentwicklungen der Funktionen $C_{n}(z)$ gewonnenen besseren Abschätzungen nach Satz 2.3.4 (b) verwendet.

Numerische Untersuchungen der Genauigkeit der Riemann-Siegel-Formel lassen vermuten, daß wir $\left|R_{10}(t)\right|$ etwa um den Faktor $10^{6}$ überschätzt haben. Mit $t_{0}=200$ führt das in 3.36 noch zu einer Überschätzung von $\left|R_{K}(t)\right|$ für $K \geq 5$. Ist jedoch $K \leq 4$, so sind die in (b) angegebenen Abschätzungen von $\left|R_{K}(t)\right|$ nur geringfügig größer als $(2 \pi)^{(2 K+3) / 4}\left|C_{K+1}(z)\right| t^{-(2 K+3) / 4}$, wenn man hier $\left|C_{K+1}(z)\right|$ wieder mit seiner Abschätzung aus Satz 2.3.4(b) ersetzt. Die Abschätzungen (b) von $\left|R_{K}(t)\right|$ liegen also für $K \leq 4$ in der Größenordnung des ersten vernachlässigten Gliedes der Riemann-Siegel-Formel. Sie sind deshalb nicht mehr wesentlich verbesserbar, so daß wir sie als optimal betrachten dürfen.

Mit Hilfe der Abschätzung von $\left|\mathrm{RS}_{K}(\tau)\right|$ aus Satz 3.1.3 und einer Verallgemeinerung der Abschätzung von $|\mathrm{RU}(\tau)|$ aus Satz 4.2.4. S. 74- was grundsätzlich keine Schwierigkeiten bereitet - gelingt es, die eben für $K=10$ durchgeführte direkte Abschätzung von $\left|R_{K}(t)\right|$ auf beliebige $K \geq 0$ auszudehnen. Das führt aber zu einer sehr komplizierten und unübersichtlichen Formel, die wir hier deshalb nicht wiedergeben. Außerdem sind die in Satz 3.2.2 gewonnenen expliziten Abschätzungen der Restglieder $R_{K}(t)(0 \leq K \leq 10)$ auch für extreme numerische Ansprüche vollkommen ausreichend. Sie zeigen, daß die ersten 11 Partialsummen der Riemann-SiegelFormel - trotz der Überschätzung von $\left|R_{10}(t)\right|$ - zur Berechnung der Funktion $Z(t)$ hervorragend geeignet sind. Sämtliche mit Hilfe der Riemann-Siegel-Formel bisher durchgeführten Berechnungen von $Z(t)$ stellen sich damit nachträglich als gerechtfertigt heraus. Die Einschränkung $t \geq 200$ in Satz 3.2 .2 ist natürlich unwesentlich, da $Z(t)$ für $t<200$ gut bekannt ist und sich zudem für diese kleinen $t$ noch recht gut mit der Eulerschen Summenformel berechnen läßt.

\subsection{Zur Divergenz der Riemann-Siegel-Formel}

Wir beschließen das Kapitel mit einer kurzen Bemerkung zur Divergenz der Riemann-Siegel-Formel. Siegel schreibt in [32. S. 285], daß es - bei Verwendung unserer Notation - keineswegs trivial ist, daß $\left|R_{K}(t)\right|$ für festes $t>0$ und $K \rightarrow+\infty$ nicht gegen Null geht. Zwar ist das aufgrund der Herleitung der Riemann-Siegel-Formel in Kapitel 1 sehr wahrscheinlich; ein Beweis dafür ist aber bis heute nicht veröffentlicht worden ${ }^{7)}$ Vegen der geraden Symmetrie der Funktionen $C_{2 n}(z)$ würde es zum Nachweis der Divergenz ausreichen, Abschätzungen der Form

$$
\left|C_{2 n}(z)\right| \geq w_{2 n} \quad(n \geq 0,0 \leq z \leq 1)
$$

mit nur von $n$ abhängigen positiven Konstanten $w_{2 n}$ so anzugeben, daß die Potenzreihe $\sum_{n=0}^{\infty} w_{2 n} x^{n}$ für alle $x \neq 0$ divergiert. Das führt jedoch mit großer Sicherheit

\footnotetext{
6) Das ist möglich, da $z$ in der Lehmerschen Form der Riemann-Siegel-Formel immer der Bedingung $|z| \leq 1$ genügt.

7) Ein solcher Beweis liegt inzwischen vor, vgl. Berry [6], 1995.
} 
nicht zum Ziel, da die Funktionen $C_{2 n}(z)$ - wie man mit Hilfe ihrer Potenzreihenentwicklungen aus Tabelle IV, S. 101 leicht nachweist - für $2 n=4,8$ und 10 je eine einfache Nullstelle in dem offenen Intervall $0<z<1$ besitzen und daher bereits für diese Werte von $2 n$ nur der trivialen Abschätzung

$$
\left|C_{2 n}(z)\right| \geq 0 \quad(0 \leq z \leq 1)
$$

genügen ${ }^{8)}$ Ist jedoch in der Riemann-Siegel-Formel $q=\sqrt{\pi}$, d. h. setzt man $t=$ $t_{M}=2 \pi M^{2}(M$ ganz $>0)$, so folgt aus der Identität (vgl. Beweis von Satz 2.1.5. S. 28,

$$
\sum_{n=0}^{\infty} \widehat{C}_{2 n}(\sqrt{\pi}) \tau_{M}^{2 n} \doteq \cos \frac{\pi}{8} \sum_{n=0}^{\infty}(-1)^{n} \alpha_{2 n} \tau_{M}^{4 n}-\sin \frac{\pi}{8} \sum_{n=0}^{\infty}(-1)^{n} \alpha_{2 n+1} \tau_{M}^{4 n+2}
$$

die Divergenz der Riemann-Siegel-Formel für diese Werte von $t$; denn die beiden Potenzreihen auf der rechten Seite dieser Gleichung haben den Konvergenzradius 0, was sich ohne Schwierigkeiten mit Hilfe von Satz 4.2.4. S. 74 beweisen läßt. Damit ist die Divergenz der Riemann-Siegel-Formel zumindest für spezielle Werte von $t$ nachgewiesen.

\footnotetext{
8) Da die Funktionen $C_{n}(z)$ mit ungeradem Index grundsätzlich nur diese triviale Abschätzung
} zulassen, haben wir sie in dieser Betrachtung von vorn herein nicht betrachtet. 



\section{Kapitel 4}

\section{Hilfssätze und \\ Hilfsabschätzungen}

\subsection{Integralformeln}

Für $\Re(\tau)>0$ sei

$$
\Phi(x, \tau):=\int_{0 \nearrow 1} \frac{e^{i \pi \tau u^{2}+2 \pi i x u}}{e^{2 \pi i u}-1} d u .
$$

Dabei ist der mit $0 \nearrow 1$ bezeichnete Integrationsweg eine von links unten nach rechts oben orientierte Gerade der Steigung 1, die die reelle Achse zwischen den Punkten 0 und 1 trifft. Es gilt dann:

Satz 4.1.1. Das Integral konvergiert für jedes komplexe $x$, so daß $\Phi(x, \tau)$ eine ganze Funktion von $x$ ist und für $\tau=m / n$, wo $m$ und $n$ natürliche Zahlen sind, wird

$$
\Phi\left(x, \frac{m}{n}\right)=\frac{\sum_{k=1}^{n} e^{i \pi \frac{m}{n} k^{2}+2 k \pi i x}-\sqrt{\frac{n}{m}} e^{i \pi\left(\frac{1}{4}-\frac{n}{m} x^{2}\right)} \sum_{k=1}^{m} e^{-i \pi \frac{n}{m} k^{2}+2 k \pi i \frac{n}{m} x}}{e^{i \pi n(2 x+m)}-1} .
$$

Daher ist $\Phi(x, \tau)$ für positive rationale $\tau$ in endlicher Form durch Exponentialfunktionen darstellbar. Zum Beweis sei auf [11, Kap. V] oder [12. S. 35 ff.] verwiesen. Wir bemerken noch, daß die Nullstellen des Nenners $e^{i \pi n(2 x+m)}-1$ in der obigen Darstellung von $\Phi(x, m / n)$ gleichzeitig Nullstellen des Zähler: ${ }^{2)}$ sind. Das folgt unmittelbar aus der Tatsache, daß $\Phi(x, \tau)$ eine ganze Funktion von $x$ ist.

Satz 4.1.2. Die durch

$$
\widetilde{F}(q):=\frac{1}{2 \sqrt{\pi}} e^{i \pi / 8-i q^{2} / 2} \int_{i \sqrt{\pi} \nearrow-i \sqrt{\pi}} \frac{e^{i v^{2} / 2+q v}}{\cosh \frac{\sqrt{\pi}}{2} v} d v
$$

1) Dieses Integral ist ein spezielles „Mordell-Integral“. Definition und Eigenschaften der Mordell-Integrale, insbes. ihre Beziehung zu den Thetafunktionen, findet man in [25].

2) Daraus lassen sich auf elegante Weise die Werte der Gaußschen Summen und das quadratische Reziprozitätsgesetz herleiten, vgl. [11. Kap. V] oder [12. S. 35 ff.]. 
definierte Funktion $\widetilde{F}(q)$ ist ganz und genügt der Gleichung

$$
\widetilde{F}(q)=\frac{\cos \left(\frac{q^{2}}{2}+\frac{3 \pi}{8}\right)}{\cos \sqrt{\pi} q}+i \frac{\sqrt{2} \cos \frac{\sqrt{\pi}}{2} q-\sin \left(\frac{q^{2}}{2}+\frac{3 \pi}{8}\right)}{\cos \sqrt{\pi} q} .
$$

Der Integrationsweg ist entsprechend dem Integrationsweg $0 \nearrow 1$ in Satz $4.1 .1 z u$ verstehen. Hier trifft die Integrationsgerade daher die imaginäre Achse zwischen den Punkten $i \sqrt{\pi}$ und $-i \sqrt{\pi}$.

Beweis. Parametrisiert man den Integrationsweg mit $v=e^{i \pi / 4} u(-\infty<u<+\infty)$, so sieht man unmittelbar, daß die Konvergenz des Integrals nicht von $q$ abhängt. Folglich ist $\widetilde{F}(q)$ eine ganze Funktion von $q$.

Wir setzen in Satz 4.1.1 $m=2, n=1$ und $x=1 / 2-2 p$ mit reellem $p$. Dann ist einerseits

$$
\Phi\left(\frac{1}{2}-2 p, 2\right)=\int_{0 \nearrow 1} \frac{e^{2 \pi i u^{2}+2 \pi i(1 / 2-2 p) u}}{e^{2 \pi i u}-1} d u=\int_{0 \nearrow 1} \frac{e^{2 \pi i u^{2}-4 \pi i p u}}{e^{i \pi u}-e^{-i \pi u}} d u
$$

und andererseits

$$
\begin{aligned}
\Phi\left(\frac{1}{2}-2 p, 2\right) & =\frac{-e^{-4 \pi i p}-\frac{1}{\sqrt{2}} e^{i \pi\left(1 / 8+p-2 p^{2}\right)}\left(e^{-2 \pi i p}-e^{-4 \pi i p}\right)}{-e^{-4 \pi i p}-1} \\
& =\frac{e^{-2 \pi i p}+\frac{1}{\sqrt{2}} e^{i \pi\left(1 / 8-2 p^{2}\right)}\left(e^{i \pi p}-e^{-i \pi p}\right)}{e^{2 \pi i p}+e^{-2 \pi i p}} .
\end{aligned}
$$

Hieraus folgt

$$
2 e^{-i \pi\left(1 / 8-2 p^{2}\right)} \Phi\left(\frac{1}{2}-2 p, 2\right)=2 \frac{e^{2 \pi i\left(p^{2}-p-1 / 16\right)}+\frac{1}{\sqrt{2}}\left(e^{i \pi p}-e^{-i \pi p}\right)}{e^{2 \pi i p}+e^{-2 \pi i p}} .
$$

Daher ist

$$
\begin{gathered}
2 e^{-i \pi\left(1 / 8-2 p^{2}\right)} \int_{0 \nearrow 1} \frac{e^{2 \pi i u^{2}-4 \pi i p u}}{e^{i \pi u}-e^{-i \pi u}} d u \\
=\frac{\cos 2 \pi\left(p^{2}-p-\frac{1}{16}\right)}{\cos 2 \pi p}+i \frac{\sin 2 \pi\left(p^{2}-p-\frac{1}{16}\right)+\sqrt{2} \sin \pi p}{\cos 2 \pi p} .
\end{gathered}
$$

Geht man hier zum konjugiert Komplexen über, so wird

$$
\begin{gathered}
2 e^{i \pi\left(1 / 8-2 p^{2}\right)} \int_{0 \nwarrow 1} \frac{e^{-2 \pi i u^{2}+4 \pi i p u}}{e^{i \pi u}-e^{-i \pi u}} d u \\
=\frac{\cos 2 \pi\left(p^{2}-p-\frac{1}{16}\right)}{\cos 2 \pi p}-i \frac{\sin 2 \pi\left(p^{2}-p-\frac{1}{16}\right)+\sqrt{2} \sin \pi p}{\cos 2 \pi p}
\end{gathered}
$$

und durch holomorphe Fortsetzung bleibt das für alle komplexen p richtig. (Der Integrationsweg $0 \nwarrow 1$ ist wieder entsprechend dem Integrationsweg $0 \nearrow 1 \mathrm{zu}$ verstehen. Die Steigung der Integrationsgeraden beträgt hier also -1). 
Setzen wir jetzt $p=(1-q / \sqrt{\pi}) / 2$ und substituieren im Integral mit $u=$ $(1+i v / \sqrt{\pi}) / 2$, so geht der Integrationsweg $0 \nwarrow 1$ bezüglich $u$ in den Integrationsweg $i \sqrt{\pi} \nearrow-i \sqrt{\pi}$ bezüglich $v$ über. Für die linke Seite der letzten Gleichung erhalten wir dann nach einer einfachen Rechnung den Ausdruck

$$
\frac{1}{2 \sqrt{\pi}} e^{i \pi / 8-i q^{2} / 2} \int_{i \sqrt{\pi} \nearrow-i \sqrt{\pi}} \frac{e^{i v^{2} / 2+q v}}{\cosh \frac{\sqrt{\pi}}{2} v} d v
$$

und für die rechte Seite dieser Gleichung

$$
\frac{\cos \left(\frac{q^{2}}{2}+\frac{3 \pi}{8}\right)}{\cos \sqrt{\pi} q}+i \frac{\sqrt{2} \cos \frac{\sqrt{\pi}}{2} q-\sin \left(\frac{q^{2}}{2}+\frac{3 \pi}{8}\right)}{\cos \sqrt{\pi} q} .
$$

Damit ist der Satz bewiesen.

Satz 4.1.3. Für jedes komplexe q ist

$$
\widetilde{F}(q)=\sqrt{2} e^{i \pi / 8} \int_{0}^{\infty} e^{-\pi v^{2} / 2} \frac{\cosh \left(\sqrt{\pi} q e^{-i \pi / 4} v\right)}{\cosh \left(\pi e^{-i \pi / 4} v\right)} d v
$$

wo $\widetilde{F}(q)$ die Funktion aus Satz 4.1.2 ist.

Beweis. Wir setzen in Satz $4.1 .1 m=1, n=2$ und erhalten

$$
\Phi\left(x, \frac{1}{2}\right)=\frac{i+e^{2 \pi i x}-\sqrt{2} e^{i \pi\left(1 / 4+2 x-2 x^{2}\right)}}{e^{2 \pi i x}-e^{-2 \pi i x}} .
$$

Für $x=z / 2+1 / 4$ wird hieraus nach Multiplikation mit $i \sqrt{2} e^{-i \pi z / 2}$

$$
\begin{aligned}
i \sqrt{2} e^{-i \pi z / 2} \Phi\left(\frac{z}{2}+\frac{1}{4}, \frac{1}{2}\right) & =i \sqrt{2} \frac{e^{i \pi z / 2}+e^{-i \pi z / 2}-\sqrt{2} e^{i \pi\left(1 / 8-z^{2} / 2\right)}}{e^{i \pi z}+e^{-i \pi z}} \\
& =\frac{\sin \frac{\pi}{2}\left(\frac{1}{4}-z^{2}\right)}{\cos \pi z}+i \frac{\sqrt{2} \cos \frac{\pi}{2} z-\cos \frac{\pi}{2}\left(\frac{1}{4}-z^{2}\right)}{\cos \pi z} \\
& =\frac{\cos \frac{\pi}{2}\left(z^{2}+\frac{3}{4}\right)}{\cos \pi z}+i \frac{\sqrt{2} \cos \frac{\pi}{2} z-\sin \frac{\pi}{2}\left(z^{2}+\frac{3}{4}\right)}{\cos \pi z} .
\end{aligned}
$$

Andererseits haben wir nach Satz 4.1.1 die Integraldarstellung

$$
\begin{aligned}
i \sqrt{2} e^{-i \pi z / 2} \Phi\left(\frac{z}{2}+\frac{1}{4}, \frac{1}{2}\right) & =i \sqrt{2} e^{-i \pi z / 2} \int_{0 \nearrow 1} \frac{e^{i \pi u^{2} / 2+i \pi z u+i \pi u / 2}}{e^{2 \pi i u}-1} d u \\
& =\frac{1}{\sqrt{2}} e^{-i \pi z / 2} \int_{0 \nearrow 1} \frac{e^{i \pi u^{2} / 2+i \pi z u-i \pi u / 2}}{\sin \pi u} d u .
\end{aligned}
$$


Hier können wir den Integrationsweg durch den Punkt 1/2 legen. Wir parametrisieren den Weg mit $u=1 / 2+e^{i \pi / 4} v$ und erhalten nach einer einfachen Rechnung

$$
\begin{aligned}
& =\frac{1}{\sqrt{2}} e^{i \pi / 8} \int_{-\infty}^{+\infty} \frac{e^{-\pi v^{2} / 2+i \pi z e^{i \pi / 4} v}}{\cos \left(\pi e^{i \pi / 4} v\right)} d v \\
& =\sqrt{2} e^{i \pi / 8} \int_{0}^{\infty} e^{-\pi v^{2} / 2} \frac{\cos \left(\pi z e^{i \pi / 4} v\right)}{\cos \left(\pi e^{i \pi / 4} v\right)} d v .
\end{aligned}
$$

Dabei ergibt sich der letzte Ausdruck aus dem vorangehenden, wenn man dort das Integral in $\int_{-\infty}^{0}+\int_{0}^{\infty}$ zerlegt und in $\int_{-\infty}^{0} v$ mit $-v$ substituiert. Durch Vergleich mit dem obenstehenden Ausdruck für $i \sqrt{2} e^{-i \pi z / 2} \Phi(z / 2+1 / 4,1 / 2)$ folgt hieraus, wenn man noch $\cos \left(e^{i \pi / 4} x\right)=\cosh \left(e^{-i \pi / 4} x\right)$ beachtet, die für alle komplexen $z$ geltende Gleichung

$$
\begin{aligned}
& \frac{\cos \frac{\pi}{2}\left(z^{2}+\frac{3}{4}\right)}{\cos \pi z}+i \frac{\sqrt{2} \cos \frac{\pi}{2} z-\sin \frac{\pi}{2}\left(z^{2}+\frac{3}{4}\right)}{\cos \pi z} \\
& =\sqrt{2} e^{i \pi / 8} \int_{0}^{\infty} e^{-\pi v^{2} / 2} \frac{\cosh \left(\pi z e^{-i \pi / 4} v\right)}{\cosh \left(\pi e^{-i \pi / 4} v\right)} d v
\end{aligned}
$$

Setzt man hier $z=q / \sqrt{\pi}$, so wird die linke Seite dieser Gleichung nach Satz 4.1 .2 gleich der Funktion $\widetilde{F}(q)$ und es ergibt sich die Behauptung.

Satz 4.1.4. Für reelle $z$ besitzt die Funktion

$$
F(z):=\frac{\cos \frac{\pi}{2}\left(z^{2}+\frac{3}{4}\right)}{\cos \pi z}
$$

die Integraldarstellung

$$
F(z)=\sqrt{2} \Re\left(e^{-i \pi / 8} \int_{0}^{\infty} e^{-\pi v^{2} / 2} \frac{\cosh \left(\pi z e^{i \pi / 4} v\right)}{\cosh \left(\pi e^{i \pi / 4} v\right)} d v\right) .
$$

Beweis. In 4.1) sei $z$ reell. Dann ist

$$
F(z)=\sqrt{2} \Re\left(e^{i \pi / 8} \int_{0}^{\infty} e^{-\pi v^{2} / 2} \frac{\cosh \left(\pi z e^{-i \pi / 4} v\right)}{\cosh \left(\pi e^{-i \pi / 4} v\right)} d v\right),
$$

und hieraus folgt die Behauptung, wenn man den Ausdruck in der großen Klammer mit seinem konjugiert Komplexen ersetzt.

Satz 4.1.5. Für die geraden Ableitungen der Funktionen

$$
\widehat{F}(q):=\frac{\cos \left(\frac{q^{2}}{2}+\frac{3 \pi}{8}\right)}{\cos \sqrt{\pi} q}
$$


4.1. Integralformeln

$$
\widehat{F}(q):=\frac{\sqrt{2} \cos \frac{\sqrt{\pi}}{2} q-\sin \left(\frac{q^{2}}{2}+\frac{3 \pi}{8}\right)}{\cos \sqrt{\pi} q}
$$

an der Stelle $q=\sqrt{\pi}$ gelten die Formeln

$$
\begin{aligned}
\widehat{F}^{(4 n)}(\sqrt{\pi}) & =(-1)^{n} \frac{(4 n) !}{2^{2 n}(2 n) !} \cos \frac{\pi}{8} \\
\widehat{F}^{(4 n+2)}(\sqrt{\pi}) & =(-1)^{n} \frac{(4 n+2) !}{2^{2 n+1}(2 n+1) !} \sin \frac{\pi}{8}, \\
\widehat{F}^{(4 n)}(\sqrt{\pi}) & =(-1)^{n} \frac{(4 n) !}{2^{2 n}(2 n) !} \sin \frac{\pi}{8} \\
\widehat{F}^{(4 n+2)}(\sqrt{\pi}) & =-(-1)^{n} \frac{(4 n+2) !}{2^{2 n+1}(2 n+1) !} \cos \frac{\pi}{8} .
\end{aligned}
$$

Beweis. Aus Satz 4.1 .3 folgt durch $2 n$-malige Differentiation nach $q$

$$
\widetilde{F}^{(2 n)}(q)=\sqrt{2} e^{i \pi / 8} \int_{0}^{\infty} e^{-\pi v^{2} / 2}\left(\sqrt{\pi} e^{-i \pi / 4} v\right)^{2 n} \frac{\cosh \left(\sqrt{\pi} q e^{-i \pi / 4} v\right)}{\cosh \left(\pi e^{-i \pi / 4} v\right)} d v .
$$

Für $q=\sqrt{\pi}$ heißt das

$$
\begin{aligned}
\widetilde{F}^{(2 n)}(\sqrt{\pi}) & =\sqrt{2} \pi^{n} i^{-n} e^{i \pi / 8} \int_{0}^{\infty} e^{-\pi v^{2} / 2} v^{2 n} d v \\
& =i^{-n} e^{i \pi / 8} \frac{2^{n}}{\sqrt{\pi}} \Gamma\left(n+\frac{1}{2}\right) \\
& =i^{-n} \frac{(2 n) !}{2^{n} n !} e^{i \pi / 8}
\end{aligned}
$$

da für ganze $n \geq 0$

$$
\Gamma\left(n+\frac{1}{2}\right)=\frac{(2 n) !}{2^{2 n} n !} \sqrt{\pi}
$$

ist. Ersetzt man jetzt $n$ zum einen mit $2 n$ und zum anderen mit $2 n+1$, so erhält man die Formeln

$$
\begin{aligned}
\widetilde{F}^{(4 n)}(\sqrt{\pi}) & =(-1)^{n} \frac{(4 n) !}{2^{2 n}(2 n) !}\left(\cos \frac{\pi}{8}+i \sin \frac{\pi}{8}\right), \\
\widetilde{F}^{(4 n+2)}(\sqrt{\pi}) & =(-1)^{n} \frac{(4 n+2) !}{2^{2 n+1}(2 n+1) !}\left(\sin \frac{\pi}{8}-i \cos \frac{\pi}{8}\right) .
\end{aligned}
$$

Durch Vergleich von Real- und Imaginärteil folgt hieraus die Behauptung, denn nach Satz 4.1.2 ist $\widetilde{F}(q)=\widehat{F}(q)+i \widehat{\hat{F}}(q)$. 
Satz 4.1.6 (Integralformel von Riemann-Siegel). Bis auf Polstellen ist für alle komplexen $s$

$$
\begin{gathered}
\pi^{-\frac{s}{2}} \Gamma\left(\frac{s}{2}\right) \zeta(s) \\
=\pi^{-\frac{s}{2}} \Gamma\left(\frac{s}{2}\right) \int_{0 \swarrow 1} \frac{e^{i \pi x^{2}} x^{-s}}{e^{i \pi x}-e^{-i \pi x}} d x+\pi^{-\frac{1-s}{2}} \Gamma\left(\frac{1-s}{2}\right) \int_{0 \searrow 1} \frac{e^{-i \pi x^{2}} x^{s-1}}{e^{i \pi x}-e^{-i \pi x}} d x .
\end{gathered}
$$

Die beiden durch die Integrale gegebenen Funktionen von $s$ sind ganz. Von den Potenzen $x^{-s}$ und $x^{s-1}$ sind die Hauptwerte zu nehmen.

Beweis. Wir setzen in Satz 4.1.1 $n=m=1$ und $x=z+1 / 2$. Nach einfacher Umformung erhält man die für alle komplexen $z$ geltende Gleichung

$$
\int_{0 \nearrow 1} \frac{e^{i \pi u^{2}+2 \pi i z u}}{e^{i \pi u}-e^{-i \pi u}} d u=\frac{e^{i \pi z}-e^{-i \pi z^{2}}}{e^{i \pi z}-e^{-i \pi z}} .
$$

Es sei $s$ eine reelle Zahl $>1$. Wir multiplizieren die letzte Gleichung mit $z^{s-1}$ und integrieren, im Nullpunkt beginnend, längs der Winkelhalbierenden des zweiten Quadranten über $z$. Dabei sei die Potenz $z^{s-1}$ die holomorphe Fortsetzung der für positive reelle $z$ reellen Funktion $z^{s-1}=e^{(s-1) \log z}$ in die längs der negativen Achse bis zum Nullpunkt aufgeschnittenen $z$-Ebene. Es wird dann

$$
\begin{gathered}
\int_{0}^{e^{i \frac{3 \pi}{4}}} \int_{0 \nearrow 1} z^{s-1} \frac{e^{i \pi u^{2}+2 \pi i z u}}{e^{i \pi u}-e^{-i \pi u}} d u d z \\
=\int_{0}^{e^{i \frac{3 \pi}{4}} \infty} z^{s-1} \frac{e^{i \pi z}}{e^{i \pi z}-e^{-i \pi z}} d z-\int_{0}^{e^{i \frac{3 \pi}{4}} \infty} z^{s-1} \frac{e^{-i \pi z^{2}}}{e^{i \pi z}-e^{-i \pi z}} d z .
\end{gathered}
$$

Das Doppelintegral auf der linken Seite dieser Gleichung konvergiert absolut ${ }^{3)}$ Daher dürfen wir hier die Integrationsreihenfolge vertauschen und erhalten so

$$
\int_{0 \nearrow 1} \frac{e^{i \pi u^{2}}}{e^{i \pi u}-e^{-i \pi u}} \int_{0}^{e^{i \frac{3 \pi}{4}} \infty} e^{2 \pi i z u} z^{s-1} d z d u .
$$

Mit der Substitution $z=e^{i 3 \pi / 4} y$ wird aber, da für $u$ auf dem Integrationsweg $0 \nearrow 1$ $\Re\left(2 \pi e^{i \pi / 4} u\right)>0$ ist

$$
\begin{aligned}
\int_{0}^{e^{i \frac{3 \pi}{4}} \infty} e^{2 \pi i z u} z^{s-1} d z & =\int_{0}^{\infty} e^{-2 \pi e^{i \pi / 4} u y}\left(e^{i 3 \pi / 4} y\right)^{s-1} e^{i 3 \pi / 4} d y \\
& =e^{i \frac{3 \pi}{4} s} \int_{0}^{\infty} e^{-2 \pi e^{i \pi / 4} u y} y^{s-1} d y
\end{aligned}
$$

\footnotetext{
3) Dazu parametrisiere man etwa $u=1 / 2+e^{i \pi v / 4}(-\infty<v<+\infty)$ und $z=e^{i 3 \pi y / 4}(0<y<$
} $+\infty)$ und betrachte den Betrag des Integranden. 


$$
\begin{aligned}
& =e^{i \frac{3 \pi}{4} s} \frac{\Gamma(s)}{\left(2 \pi e^{i \pi / 4} u\right)^{s}} \\
& =e^{i \frac{\pi}{2} s}(2 \pi)^{-s} \Gamma(s) u^{-s} .
\end{aligned}
$$

Folglich ist die linke Seite von 4.2 gleich dem Ausdruck

$$
e^{i \frac{\pi}{2} s}(2 \pi)^{-s} \Gamma(s) \int_{0 \nearrow 1} \frac{e^{i \pi u^{2}} u^{-s}}{e^{i \pi u}-e^{-i \pi u}} d u .
$$

Das zweite Integral auf der rechten Seite von 4.2 läßt sich in eine 4.3 ähnliche Form bringen. Dazu betrachten wir das Integral

$$
\int_{0 \nwarrow 1} z^{s-1} \frac{e^{-i \pi z^{2}}}{e^{i \pi z}-e^{-i \pi z}} d z .
$$

Wegen $s>1$ läßt sich die Integrationsgerade $0 \nwarrow 1$ durch den Nullpunkt legen. Damit wird

$$
\begin{aligned}
\int_{0 \nwarrow 1} z^{s-1} \frac{e^{-i \pi z^{2}}}{e^{i \pi z}-e^{-i \pi z}} d z & =\int_{-e^{i \frac{3 \pi}{4}} \infty}^{e^{i \frac{3 \pi}{4}} \infty} z^{s-1} \frac{e^{-i \pi z^{2}}}{e^{i \pi z}-e^{-i \pi z}} d z \\
& =\int_{0}^{e^{i \frac{3 \pi}{4}} \infty} z^{s-1} \frac{e^{-i \pi z^{2}}}{e^{i \pi z}-e^{-i \pi z}} d z+\int_{-e^{i \frac{3 \pi}{4}} \infty}^{0} \frac{e^{(s-1) \log z-i \pi z^{2}}}{e^{i \pi z}-e^{-i \pi z}} d z .
\end{aligned}
$$

Für $\Im(z)>0$ gilt nach Festlegung der Potenz $z^{s-1}=e^{(s-1) \log z}$ die Gleichung $\log (-z)=\log z-i \pi$ und daher

$$
\begin{aligned}
\int_{-e^{i \frac{3 \pi}{4}} \infty}^{0} \frac{e^{(s-1) \log z-i \pi z^{2}}}{e^{i \pi z}-e^{-i \pi z}} d z & =-\int_{0}^{e^{i \frac{3 \pi}{4}} \infty} e^{(s-1) \log (-z)} \frac{e^{-i \pi z^{2}}}{e^{i \pi z}-e^{-i \pi z}} d z \\
& =e^{-i \pi s} \int_{0}^{e^{i \frac{3 \pi}{4}} \infty} \frac{e^{-i \pi z^{2}} z^{s-1}}{e^{i \pi z}-e^{-i \pi z}} d z .
\end{aligned}
$$

Wir setzen das oben ein und erhalten für das zweite Integral auf der rechten Seite von 4.2

$$
\int_{0}^{e^{i \frac{3 \pi}{4}}} \frac{e^{-i \pi z^{2}} z^{s-1}}{e^{i \pi z}-e^{-i \pi z}} d z=\frac{1}{1+e^{-i \pi s}} \int_{0 \nwarrow 1} \frac{e^{-i \pi z^{2}} z^{s-1}}{e^{i \pi z}-e^{-i \pi z}} d z .
$$

Das erste Integral auf der rechten Seite von 4.2 läßt sich wie folgt berechnen:

$$
\int_{0}^{e^{i \frac{3 \pi}{4}} \infty} z^{s-1} \frac{e^{i \pi z}}{e^{i \pi z}-e^{-i \pi z}} d z=-\int_{0}^{e^{i \frac{3 \pi}{4}} \infty} z^{s-1} \sum_{n=1}^{\infty} e^{2 n \pi i z} d z .
$$


Hier sind Summation und Integration vertauschbar; also

$$
\begin{aligned}
& =-\sum_{n=1}^{\infty} \int_{0}^{e^{i \frac{3 \pi}{4}} e^{2 n \pi i z} z^{s-1} d z} \\
& =-\sum_{n=1}^{\infty} \int_{0}^{\infty} e^{-2 n \pi e^{i \pi / 4} y}\left(e^{i 3 \pi / 4} y\right)^{s-1} e^{i 3 \pi / 4} d y \\
& =-e^{i \frac{3 \pi}{4} s} \sum_{n=1}^{\infty} \frac{\Gamma(s)}{\left(2 n \pi e^{i \pi / 4}\right)^{s}} \\
& =-e^{i \frac{\pi}{2} s}(2 \pi)^{-s} \Gamma(s) \sum_{n=1}^{\infty} \frac{1}{n^{s}} \\
& =-e^{i \frac{\pi}{2} s}(2 \pi)^{-s} \Gamma(s) \zeta(s) .
\end{aligned}
$$

Setzen wir das zusammen mit 4.3 und 4.4 in 4.2 ein, so wird

$$
\begin{gathered}
e^{i \frac{\pi}{2} s}(2 \pi)^{-s} \Gamma(s) \int_{0 \nearrow 1} \frac{e^{i \pi x^{2}} x^{-s}}{e^{i \pi x}-e^{-i \pi x}} d x \\
=-e^{i \frac{\pi}{2} s}(2 \pi)^{-s} \Gamma(s) \zeta(s)-\frac{1}{1+e^{-i \pi s}} \int_{0 \nwarrow 1} \frac{e^{-i \pi x^{2}} x^{s-1}}{e^{i \pi x}-e^{-i \pi x}} d x .
\end{gathered}
$$

Daraus folgt

$$
\begin{gathered}
(2 \pi)^{-s} \Gamma(s) \zeta(s) \\
=(2 \pi)^{-s} \Gamma(s) \int_{0 \swarrow 1} \frac{e^{i \pi x^{2}} x^{-s}}{e^{i \pi x}-e^{-i \pi x}} d x+\frac{e^{-i \frac{\pi}{2} s}}{1+e^{-i \pi s}} \int_{0 \searrow 1} \frac{e^{-i \pi x^{2}} x^{s-1}}{e^{i \pi x}-e^{-i \pi x}} d x .
\end{gathered}
$$

Es ist aber nach dem Ergänzungssatz der Gammafunktion

$$
\frac{e^{-i \frac{\pi}{2} s}}{1+e^{-i \pi s}}=\frac{1}{2 \cos \frac{\pi}{2} s}=\frac{1}{2 \pi} \Gamma\left(\frac{1-s}{2}\right) \Gamma\left(\frac{1+s}{2}\right)
$$

und nach dem Verdoppelungssatz

$$
(2 \pi)^{-s} \Gamma(s)=\pi^{-\frac{s}{2}} \Gamma\left(\frac{s}{2}\right) \frac{\pi^{\frac{1-s}{2}}}{2 \pi} \Gamma\left(\frac{1+s}{2}\right) .
$$

Trägt man das in die letzte Gleichung ein, so ergibt sich nach Multiplikation mit $\pi^{-(1-s) / 2}$ und nach Kürzen von $\Gamma[(1+s) / 2] /(2 \pi)$ die Behauptung des Satzes. Die Einschränkung $s$ reell $>1$ kann natürlich jetzt fortfallen; denn da die Konvergenz der beiden Integrale in der Riemann-Siegel-Integralformel offenbar nicht von $s$ abhängt, sind diese Integrale ganze Funktionen von $s$. Folglich ist die rechte Seite der Riemann-Siegel-Integralformel wegen der Meromorphie der Gammafunktion in die 
ganze Ebene meromorph fortsetzbar. Aufgrund der Art der Herleitung ist ferner unmittelbar ersichtlich, daß von den Potenzen $z^{-s}$ und $z^{s-1}$ die Hauptwerte zu nehmen sind. Damit ist der Satz bewiesen.

Eine ähnliche Herleitung der Riemann-Siegel-Integralformel findet sich in 16. S. 166] und in [31, 32].

\subsection{Die asymptotische Entwicklung von $\vartheta(t)$}

Wie N. Nielsen in [26, S. 87] setzen wir in der längs der negativen reellen Achse bis zum Nullpunkt aufgeschnittenen $z$-Ebene

$$
\mu(z):=\log \Gamma(z)-\left(z-\frac{1}{2}\right) \log z+z-\log \sqrt{2 \pi} .
$$

Dabei sind die rechtsstehenden Logarithmen so zu bestimmen, daß $\mu(z)$ für positive reelle $z$ reell wird.

Satz 4.2.1. Die oben eingeführte Funktion $\mu(z)$ hat folgende Eigenschaften:

(a) $\mu(\bar{z})=\overline{\mu(z)}$

(b) $\mu(z)=\mu(z+1)+\left(z+\frac{1}{2}\right) \log \left(1+\frac{1}{z}\right)-1$

(c) $\mu(z)+\mu\left(z+\frac{1}{2}\right)=\mu(2 z)-z \log \left(1+\frac{1}{2 z}\right)+\frac{1}{2}$

(d) $\mu(z)+\mu(-z)=-\log \left(1-e^{2 \pi i z \operatorname{sign} \Im(z)}\right) \quad$ (z nicht reell)

(e) (Stirlingsche Reihe)

Für $K \geq 0$ und jedes $\varepsilon>0$ ist

$$
\mu(z)=\sum_{n=1}^{K} \frac{B_{2 n}}{(2 n-1) 2 n z^{2 n-1}}+O\left(z^{-2 K-1}\right) \quad z \rightarrow \infty \text { in }|\arg z| \leq \pi-\varepsilon
$$

Die $B_{2 n}$ sind die Bernoullischen Zahlen.

(f) (Restabschätzung der Stirlingschen Reihe)

Für $\Re(z) \geq 0$ und $K \geq 1$ gilt für das Restglied

$$
r_{K}(z):=\mu(z)-\sum_{n=1}^{K} \frac{B_{2 n}}{(2 n-1) 2 n z^{2 n-1}}
$$

die Abschätzung

$$
\left|r_{K}(z)\right| \leq \frac{\left|B_{2 K+2}\right|}{(2 K+1)(2 K+2)|z|^{2 K+1}}\left(1+\frac{2 K+1}{2} \sqrt{\frac{\pi}{K}}\right) .
$$

In allen Fällen ist von den Logarithmen der Hauptwert zu nehmen. 
Zum Beweis bemerken wir, daß (a) unmittelbar aus 4.5 folgt. Die Gleichung (b) ergibt sich aus der Funktionalgleichung, (c) aus dem Verdopplungs- und (d) aus dem Ergänzungssatz der Gammafunktion. Einen Beweis für (d) findet man z. B. in [26. S. 94 4) und für die wichtige Abschätzung (f) in [4. S. 303]. Im übrigen verweisen wir auf [4] und [26].

Satz 4.2.2. Es sei $t>0$. Dann ist

$$
\begin{gathered}
\Im\left[\left(i \frac{t}{2}-\frac{1}{4}\right) \log \left(1+\frac{1}{2 i t}\right)+\mu\left(\frac{1}{4}+i \frac{t}{2}\right)\right] \\
=\frac{1}{2} \Im[\mu(2 i t)-\mu(i t)]+\frac{1}{2} \arctan e^{-\pi t},
\end{gathered}
$$

wenn von dem Logarithmus der Hauptwert genommen wird.

Beweis. (Von allen hier auftretenden Logarithmen ist der Hauptwert zu nehmen). Wir setzen in Satz 4.2.1 $z=-1 / 4+i t / 2$. Aus (c) folgt

$$
\mu\left(-\frac{1}{4}+i \frac{t}{2}\right)+\mu\left(\frac{1}{4}+i \frac{t}{2}\right)=\mu\left(-\frac{1}{2}+i t\right)+\left(\frac{1}{4}-i \frac{t}{2}\right) \log \left(1+\frac{1}{-1 / 2+i t}\right)+\frac{1}{2}
$$

und aus $(\mathrm{d})$

$$
\mu\left(-\frac{1}{4}+i \frac{t}{2}\right)+\mu\left(\frac{1}{4}-i \frac{t}{2}\right)=-\log \left(1-e^{2 \pi i(-1 / 4+i t / 2)}\right)=-\log \left(1+i e^{-\pi t}\right) .
$$

Wir subtrahieren die zweite von der ersten Gleichung und erhalten wegen Satz 4.2 .1 (a)

$$
\begin{gathered}
\mu\left(\frac{1}{4}+i \frac{t}{2}\right)-\mu\left(\frac{1}{4}-i \frac{t}{2}\right) \\
=\mu\left(\frac{1}{4}+i \frac{t}{2}\right)-\overline{\mu\left(\frac{1}{4}+i \frac{t}{2}\right)}=2 i \Im \mu\left(\frac{1}{4}+i \frac{t}{2}\right)
\end{gathered}
$$

die Beziehung

$$
\begin{gathered}
2 i \Im \mu\left(\frac{1}{4}+i \frac{t}{2}\right) \\
=\mu\left(-\frac{1}{2}+i t\right)+\left(\frac{1}{4}-i \frac{t}{2}\right) \log \left(1+\frac{1}{-1 / 2+i t}\right)+\log \left(1+i e^{-\pi t}\right)+\frac{1}{2} .
\end{gathered}
$$

Für $z=-1 / 2+i t$ ist nach Satz 4.2 .1 (b)

$$
\mu\left(-\frac{1}{2}+i t\right)=\mu\left(\frac{1}{2}+i t\right)+i t \log \left(1+\frac{1}{-1 / 2+i t}\right)-1
$$

und für $z=i t$ nach Satz 4.2 .1 (c)

$$
\mu\left(\frac{1}{2}+i t\right)=\mu(2 i t)-\mu(i t)-i t \log \left(1+\frac{1}{2 i t}\right)+\frac{1}{2} .
$$

Zusammengefaßt lauten die beiden letzten Gleichungen

$$
\mu\left(-\frac{1}{2}+i t\right)=\mu(2 i t)-\mu(i t)-i t \log \left(1+\frac{1}{2 i t}\right)+i t \log \left(1+\frac{1}{-1 / 2+i t}\right)-\frac{1}{2} .
$$

4) In diesem Buch achte der Leser besonders auf Druckfehler! 
Wir tragen das in 4.6 ein:

$$
\begin{aligned}
2 i \Im \mu\left(\frac{1}{4}+i \frac{t}{2}\right)= & \mu(2 i t)-\mu(i t)-i t \log \left(1+\frac{1}{2 i t}\right) \\
& +\left(\frac{1}{4}+i \frac{t}{2}\right) \log \left(1+\frac{1}{-1 / 2+i t}\right)+\log \left(1+i e^{-\pi t}\right) .
\end{aligned}
$$

Da $t$ reell $>0$ ist, gilt die Zerlegung

$$
\log \left(1+\frac{1}{-1 / 2+i t}\right)=\log \left(1+\frac{1}{2 i t}\right)-\log \left(1-\frac{1}{2 i t}\right) .
$$

Damit wird

$$
\begin{aligned}
& \left(\frac{1}{4}+i \frac{t}{2}\right) \log \left(1+\frac{1}{-1 / 2+i t}\right)-i t \log \left(1+\frac{1}{2 i t}\right) \\
= & \left(\frac{1}{4}-i \frac{t}{2}\right) \log \left(1+\frac{1}{2 i t}\right)-\left(\frac{1}{4}+i \frac{t}{2}\right) \log \left(1-\frac{1}{2 i t}\right) \\
= & 2 i \Im\left[\left(\frac{1}{4}-i \frac{t}{2}\right) \log \left(1+\frac{1}{2 i t}\right)\right] .
\end{aligned}
$$

Aus 4.7 folgt dann, wenn wir gleich durch $2 i$ dividieren,

$$
\begin{aligned}
& \Im\left[\left(i \frac{t}{2}-\frac{1}{4}\right) \log \left(1+\frac{1}{2 i t}\right)+\mu\left(\frac{1}{4}+i \frac{t}{2}\right)\right] \\
& =\frac{1}{2 i}[\mu(2 i t)-\mu(i t)]+\frac{1}{2 i} \log \left(1+i e^{-\pi t}\right) .
\end{aligned}
$$

Die linke Seite dieser Gleichung ist reell. Folglich reicht es, wenn man von der rechten Seite den Realteil nimmt. Wegen

$$
\Re\left[\frac{1}{2 i} \log \left(1+i e^{-\pi t}\right)\right]=\frac{1}{2} \arctan e^{-\pi t}
$$

ergibt sich daraus die Behauptung.

Satz 4.2.3. Für reelle $t$ sei

$$
\vartheta(t):=\Im \log \Gamma\left(\frac{1}{4}+i \frac{t}{2}\right)-\frac{t}{2} \log \pi .
$$

Von dem Logarithmus ist der Hauptwert zu nehmen, so daß $\vartheta(t)$ bei $t=0$ verschwindet. Dann ist für $t>0$ :

(a) $\vartheta(t)=\frac{t}{2} \log \frac{t}{2 \pi}-\frac{t}{2}-\frac{\pi}{8}+\frac{1}{2} \arctan e^{-\pi t}+\frac{1}{2} \Im[\mu(2 i t)-\mu(i t)]$

(b) $\vartheta(t)=\frac{t}{2} \log \frac{t}{2 \pi}-\frac{t}{2}-\frac{\pi}{8}+\sum_{n=1}^{K} \frac{\left(2^{2 n-1}-1\right)\left|B_{2 n}\right|}{2^{2 n}(2 n-1) 2 n t^{2 n-1}}+O\left(t^{-2 K-1}\right) \quad \begin{gathered}(t \rightarrow+\infty \\ K \geq 0)\end{gathered}$

(c) $\vartheta(t)$ besitzt die asymptotische Entwicklung

$$
\vartheta(t) \sim \frac{t}{2} \log \frac{t}{2 \pi}-\frac{t}{2}-\frac{\pi}{8}+\sum_{n=1}^{\infty} \frac{\left(2^{2 n-1}-1\right)\left|B_{2 n}\right|}{2^{2 n}(2 n-1) 2 n t^{2 n-1}} \quad(t \rightarrow+\infty) .
$$


(d) In der Darstellung

$$
\vartheta(t)=\frac{t}{2} \log \frac{t}{2 \pi}-\frac{t}{2}-\frac{\pi}{8}+\frac{1}{48 t}+\frac{7}{5760 t^{3}}+\frac{31}{80640 t^{5}}+R \vartheta(t)
$$

genügt das Restglied $R \vartheta(t)$ für $t \geq 10$ der Abschätzung

$$
|R \vartheta(t)|<\frac{1}{3322 t^{7}}
$$

Beweis. Es sei $t$ reell $>0$. Aus 4.5 folgt für $z=1 / 4+i t / 2$

$$
\log \Gamma\left(\frac{1}{4}+i \frac{t}{2}\right)=\left(i \frac{t}{2}-\frac{1}{4}\right) \log \left(\frac{1}{4}+i \frac{t}{2}\right)-\frac{1}{4}-i \frac{t}{2}+\log \sqrt{2 \pi}+\mu\left(\frac{1}{4}+i \frac{t}{2}\right) .
$$

Wegen

$$
\log \left(\frac{1}{4}+i \frac{t}{2}\right)=\log i \frac{t}{2}+\log \left(1+\frac{1}{2 i t}\right)=\log \frac{t}{2}+i \frac{\pi}{2}+\log \left(1+\frac{1}{2 i t}\right)
$$

wird dann

$$
\begin{gathered}
\Im \log \Gamma\left(\frac{1}{4}+i \frac{t}{2}\right) \\
=\Im\left[\left(i \frac{t}{2}-\frac{1}{4}\right)\left(\log \frac{t}{2}+i \frac{\pi}{2}\right)-\frac{1}{4}-i \frac{t}{2}+\log \sqrt{2 \pi}\right. \\
\left.+\left(i \frac{t}{2}-\frac{1}{4}\right) \log \left(1+\frac{1}{2 i t}\right)+\mu\left(\frac{1}{4}+i \frac{t}{2}\right)\right] \\
=\frac{t}{2} \log \frac{t}{2}-\frac{t}{2}-\frac{\pi}{8}+\Im\left[\left(i \frac{t}{2}-\frac{1}{4}\right) \log \left(1+\frac{1}{2 i t}\right)+\mu\left(\frac{1}{4}+i \frac{t}{2}\right)\right] .
\end{gathered}
$$

Hieraus ergibt sich (a), wenn man auf beiden Seiten der Gleichung $(t \log \pi) / 2$ abzieht und Satz 4.2 .2 anwendet.

Mit $z=i t$ lautet (f) von Satz 4.2 .1 wegen $\left|B_{2 n}\right|=(-1)^{n-1} B_{2 n}$

$$
\begin{aligned}
\mu(i t) & =\sum_{n=1}^{K} \frac{B_{2 n}}{(2 n-1) 2 n(i t)^{2 n-1}}+r_{K}(i t) \\
& =i \sum_{n=1}^{K} \frac{(-1)^{n} B_{2 n}}{(2 n-1) 2 n t^{2 n-1}}+r_{K}(i t) \quad(K \geq 1) \\
& =-i \sum_{n=1}^{K} \frac{\left|B_{2 n}\right|}{(2 n-1) 2 n t^{2 n-1}}+r_{K}(i t) .
\end{aligned}
$$

Daher ist

$$
\begin{gathered}
\frac{1}{2} \Im[\mu(2 i t)-\mu(i t)] \\
=\frac{1}{2} \sum_{n=1}^{K} \frac{\left|B_{2 n}\right|}{(2 n-1) 2 n t^{2 n-1}}-\frac{1}{2} \sum_{n=1}^{K} \frac{\left|B_{2 n}\right|}{(2 n-1) 2 n(2 t)^{2 n-1}}+\frac{1}{2} \Im\left[r_{K}(2 i t)-r_{K}(i t)\right] \\
=\sum_{n=1}^{K} \frac{\left(2^{2 n-1}-1\right)\left|B_{2 n}\right|}{2^{2 n}(2 n-1) 2 n t^{2 n-1}}+\frac{1}{2} \Im\left[r_{K}(2 i t)-r_{K}(i t)\right] .
\end{gathered}
$$


Aus (a) folgt so die Darstellung

$$
\begin{gathered}
\vartheta(t)=\frac{t}{2} \log \frac{t}{2 \pi}-\frac{t}{2}-\frac{\pi}{8}+\frac{1}{2} \arctan e^{-\pi t} \\
+\sum_{n=1}^{K} \frac{\left(2^{2 n-1}-1\right)\left|B_{2 n}\right|}{2^{2 n}(2 n-1) 2 n t^{2 n-1}}+\frac{1}{2} \Im\left[r_{K}(2 i t)-r_{K}(i t)\right] .
\end{gathered}
$$

Nach Satz 4.2.1 (e) und (f) ist $r_{K}(2 i t)-r_{K}(i t)=O\left(t^{-2 K-1}\right)$ für $t \rightarrow+\infty$. Setzt man leere Summen gleich Null, so bleibt das auch noch für $K=0$ richtig. Da $\arctan e^{-\pi t}=O\left(t^{-K}\right)(t \rightarrow+\infty)$ für jedes $K$ ist, sind damit (b) und (c) bewiesen.

Zum Beweis von (d) wenden wir (4.8) mit $K=5$ an. Wir erhalten mit den numerischen Werten von $\left(2^{2 n-1}-1\right) \mid\left\langle B_{2 n}\right| /\left[2^{2 n}(2 n-1) 2 n\right](1 \leq n \leq 5)$

$$
\vartheta(t)=\frac{t}{2} \log \frac{t}{2 \pi}-\frac{t}{2}-\frac{\pi}{8}+\frac{1}{48 t}+\frac{7}{5760 t^{3}}+\frac{31}{80640 t^{5}}+R \vartheta(t)
$$

mit

$$
R \vartheta(t)=\frac{127}{430080 t^{7}}+\frac{511}{1216512 t^{9}}+\frac{1}{2} \arctan e^{-\pi t}+\frac{1}{2} \Im\left[r_{5}(2 i t)-r_{5}(i t)\right] .
$$

Für $t \geq 10$ läßt sich das Restglied $R \vartheta(t)$ wie folgt abschätzen:

$$
|R \vartheta(t)| \leq \frac{127}{430080 t^{7}}+\frac{511 \cdot 10^{-2}}{1216512 t^{7}}+\frac{1}{2} \arctan e^{-\pi t}+\frac{1}{2}\left|r_{5}(2 i t)\right|+\frac{1}{2}\left|r_{5}(i t)\right| .
$$

Es gelten die Abschätzungen $(t \geq 10)$

$$
\frac{1}{2} \arctan e^{-\pi t}<\frac{e^{-\pi t}}{2}<\frac{10^{-6}}{t^{7}}
$$

und wegen $\left|B_{12}\right|=691 / 2730$ nach Satz 4.2 .1 (f)

$$
\begin{aligned}
& \frac{1}{2}\left|r_{5}(2 i t)\right|+\frac{1}{2}\left|r_{5}(i t)\right| \\
& \leq \frac{1}{2} \cdot \frac{\left|B_{12}\right|}{11 \cdot 12(2 t)^{11}}\left(1+\frac{11}{2} \sqrt{\frac{\pi}{5}}\right)+\frac{1}{2} \cdot \frac{\left|B_{12}\right|}{11 \cdot 12 t^{11}}\left(1+\frac{11}{2} \sqrt{\frac{\pi}{5}}\right) \\
& =\frac{\left(2^{11}+1\right) \cdot 691}{11 \cdot 12 \cdot 2^{12} \cdot 2730 t^{11}}\left(1+\frac{11}{2} \sqrt{\frac{\pi}{5}}\right) \\
& \leq \frac{\left(2^{11}+1\right) \cdot 691}{11 \cdot 12 \cdot 2^{12} \cdot 2730 \cdot 10^{4} t^{7}}\left(1+\frac{11}{2} \sqrt{\frac{\pi}{5}}\right)<\frac{5.2 \cdot 10^{-7}}{t^{7}} .
\end{aligned}
$$

Damit wird

$$
|R \vartheta(t)|<\left[\frac{127}{430080}+\frac{511 \cdot 10^{-2}}{1216512}+10^{-6}+5.2 \cdot 10^{-7}\right] \frac{1}{t^{7}} .
$$

Hier läßt sich die Konstante in der eckigen Klammer mit 1/3322 nach oben abschätzen, wie in (d) behauptet wurde.

Wir bemerken noch, daß diese Abschätzung fast optimal ist; denn wegen

$$
\frac{127}{430080 t^{7}}=\frac{1}{3386.45 \ldots \cdot t^{7}}
$$

ist der mit $1 / 3322 t^{-7}$ abgeschätzte Fehler nur wenig größer als das erste vernachlässigte Glied der asymptotischen Reihe. 
Eine andere Art der Herleitung der asymptotischen Entwicklung (c) findet der Leser bei C. L. Siegel in [31, 32].

Satz 4.2.4. Es sei $\tau>0$ und $t=1 /\left(8 \tau^{2}\right)$. Für den durch

$$
U:=\exp i\left(\frac{t}{2} \log \frac{t}{2 \pi}-\frac{t}{2}-\frac{\pi}{8}-\vartheta(t)\right)
$$

definierten Ausdruck bestehen die Beziehungen

(a) $U=\exp \left(-i \sum_{n=1}^{K} \frac{\left(2^{2 n-1}-1\right)\left|B_{2 n}\right|}{2^{2 n}(2 n-1) 2 n}\left(8 \tau^{2}\right)^{2 n-1}\right)+O\left(\tau^{4 K+2}\right) \quad \begin{aligned} & (\tau \rightarrow 0 \\ & K \geq 0) .\end{aligned}$

(b) Für $\tau \leq 1 / 40$ genügt das Restglied $\mathrm{RU}(\tau)$ in der Darstellung

$$
U=e^{-i\left(\frac{\tau^{2}}{6}+\frac{28}{45} \tau^{6}+\frac{3968}{315} \tau^{10}\right)}+\mathrm{RU}(\tau)
$$

der Abschätzung

$$
|\operatorname{RU}(\tau)|<\frac{\tau^{11}}{101}
$$

(c) U ist in eine formale Potenzreihe der Form

$$
U \sim \sum_{n=0}^{\infty} i^{n} \alpha_{n} \tau^{2 n} \quad(\tau \rightarrow 0)
$$

entwickelbar. Für $K \geq 0$ ist

$$
U=\sum_{n=0}^{K} i^{n} \alpha_{n} \tau^{2 n}+O\left(\tau^{2 K+2}\right) \quad(\tau \rightarrow 0) .
$$

Die Koeffizienten $\alpha_{n}$ sind rationale Zahlen. Sie genügen der Rekursionsformel

$$
\begin{gathered}
\alpha_{0}=1 \\
n \alpha_{n}=-\sum_{k=1}^{\lfloor(n+1) / 2\rfloor} \beta_{k} \alpha_{n+1-2 k} \quad(n \geq 1)
\end{gathered}
$$

mit

$$
\beta_{k}:=\frac{2^{4 k-4}\left(2^{2 k-1}-1\right) B_{2 k}}{k} \quad(k \geq 1) .
$$

Für die ersten $\alpha_{n}$ ergeben sich folgende Werte:

$$
\alpha_{0}=1
$$

$$
\begin{array}{ll}
\alpha_{1}=-\frac{1}{2 \cdot 3}, & \alpha_{5}=-\frac{8689 \cdot 9463}{2^{8} \cdot 3^{6} \cdot 5 \cdot 7}, \\
\alpha_{2}=\frac{1}{2^{3} \cdot 3^{2}}, & \alpha_{6}=\frac{19 \cdot 141865649}{2^{10} \cdot 3^{8} \cdot 5^{2} \cdot 7},
\end{array}
$$


4.2. Die asymptotische Entwicklung von $\vartheta(t)$

$$
\begin{aligned}
\alpha_{3} & =\frac{4027}{2^{4} \cdot 3^{4} \cdot 5}, & \alpha_{7} & =\frac{19^{2} \cdot 47 \cdot 173 \cdot 1487809}{2^{11} \cdot 3^{9} \cdot 5^{2} \cdot 7}, \\
\alpha_{4} & =-\frac{23 \cdot 701}{2^{7} \cdot 3^{5} \cdot 5}, & \alpha_{8} & =-\frac{43 \cdot 853 \cdot 31393 \cdot 32653}{2^{15} \cdot 3^{10} \cdot 5^{2} \cdot 7} .
\end{aligned}
$$

Zähler und Nenner sind vollständig in Primfaktoren zerlegt.

Beweis. Aus Satz 4.2 .3 (b) folgt nach Definition von $U$

$$
\begin{array}{rlr}
U & =\exp \left(-i \sum_{n=1}^{K} \frac{\left(2^{2 n-1}-1\right)\left|B_{2 n}\right|}{2^{2 n}(2 n-1) 2 n t^{2 n-1}}+O\left(t^{-2 K-1}\right)\right) \quad(t \rightarrow+\infty) \\
& =\exp \left(-i \sum_{n=1}^{K} \frac{\left(2^{2 n-1}-1\right)\left|B_{2 n}\right|}{2^{2 n}(2 n-1) 2 n t^{2 n-1}}\right)+O\left(t^{-2 K-1}\right) \quad(t \rightarrow+\infty)
\end{array}
$$

und hieraus ergibt sich (a), wenn man $t=1 /\left(8 \tau^{2}\right)$ setzt.

Zum Beweis von (b) verwenden wir die Abschätzung 4.2.3(d). Wir erhalten

$$
U=e^{-i\left(\frac{1}{48 t}+\frac{7}{5760 t^{3}}+\frac{31}{80640 t^{5}}+R \vartheta(t)\right)} .
$$

Für $t=1 /\left(8 \tau^{2}\right)$ heißt das

$$
U=e^{-i\left(\frac{\tau^{2}}{6}+\frac{28}{45} \tau^{6}+\frac{3968}{315} \tau^{10}\right)}+\mathrm{RU}(\tau)
$$

mit

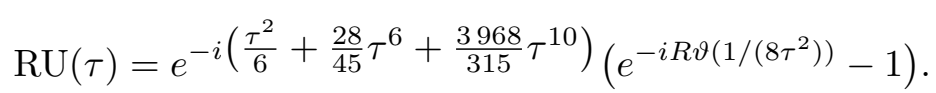

Daher ist

$$
|\mathrm{RU}(\tau)|=\left|e^{-i R \vartheta\left(1 /\left(8 \tau^{2}\right)\right)}-1\right| \leq \sum_{n=1}^{\infty} \frac{1}{n !}\left|R \vartheta\left(\frac{1}{8 \tau^{2}}\right)\right|^{n} .
$$

Es sei jetzt $\tau \leq 1 / 40$. Dann wird $1 /\left(8 \tau^{2}\right) \geq 200$ und die Abschätzung (d) aus Satz 4.2.3 ist anwendbar. Wir erhalten damit

$$
\left|R \vartheta\left(\frac{1}{8 \tau^{2}}\right)\right|<\frac{\left(8 \tau^{2}\right)^{7}}{3322}=\frac{2^{21}}{3322} \tau^{14}<632 \tau^{14}
$$

und so

$$
|\mathrm{RU}(\tau)|<\sum_{n=1}^{\infty} \frac{632^{n}}{n !} \tau^{14 n}=\tau^{11} \sum_{n=1}^{\infty} \frac{632^{n}}{n !} \tau^{14 n-11} \leq \tau^{11} \sum_{n=1}^{\infty} \frac{632^{n}}{40^{14 n-11} n !} .
$$

Da sich hier die ganz rechts stehende unendliche Reihe mit 1/101 nach oben abschätzen läßt, ist (b) bewiesen.

Zum Beweis von (c) bemerken wir, daß aus Satz 4.2.3 (c) folgt

$$
U \sim \exp \left(-i \sum_{n=1}^{\infty} \frac{\left(2^{2 n-1}-1\right)\left|B_{2 n}\right|}{2^{2 n}(2 n-1) 2 n}\left(8 \tau^{2}\right)^{2 n-1}\right) \quad(\tau \rightarrow 0) .
$$

\footnotetext{
5) In der Originalarbeit steht hier im Zähler statt „19² . . “ fälschlicherweise „19 · ...".
} 
Durch Einsetzen des Exponenten in die Potenzreihe der Exponentialfunktion erhält man hieraus mit gewissen Zahlen $\alpha_{n}$ eine formale Entwicklung von $U$ nach Potenzen von $\tau^{2}$

$$
U \sim \sum_{n=0}^{\infty} i^{n} \alpha_{n} \tau^{2 n} \quad(\tau \rightarrow 0)
$$

und nach (a) ist klar, daß jede Partialsumme dieser Reihe der in (c) angegebenen Groß-O-Abschätzung genügt.

Die Rekursionsformel der $\alpha_{n}$ läßt sich in bekannter Weise durch Gleichsetzen der logarithmischen Ableitungen von (4.9) und 4.10) gewinnen. Unter Verwendung der Beziehung $\left|B_{2 k}\right|=(-1)^{k-1} B_{2 k}$ kann man sie in die obenstehende Form bringen. Dabei ist unter $\lfloor(n+1) / 2\rfloor$ wie üblich die größte ganze Zahl $\leq(n+1) / 2$ zu verstehen. Da $U \sim 1$ für $\tau \rightarrow 0$ ist, muß $\alpha_{0}=1$ sein. Aus der Rekursionsformel erhält man damit die angegebenen Werte der $\alpha_{n}(n \leq 8)$. Es ist trivial, daß die $\alpha_{n}$ rationale Zahlen sind.

\subsection{Bestimmung von $\mathcal{D}_{0}(\tau)$ und $\mathcal{D}_{1}(\tau)$}

Satz 4.3.1. Für die formalen Potenzreihen $\mathcal{D}_{0}(\tau)$ und $\mathcal{D}_{1}(\tau)$ in

$$
U \cdot S \sim \sum_{n=0}^{\infty} \mathcal{D}_{n}(\tau) \frac{\widetilde{F}^{(n)}(q)}{n !} \quad(\tau \rightarrow 0)
$$

gilt

$$
\mathcal{D}_{0}(\tau) \doteq \sum_{n=0}^{\infty} \lambda_{n} \tau^{4 n}
$$

und

$$
\mathcal{D}_{1}(\tau) \doteq \sum_{n=1}^{\infty} \mu_{n} \tau^{4 n-1} .
$$

Die Koeffizienten sind rekursiv durch

$$
\begin{aligned}
\lambda_{0} & =1, \\
(n+1) \lambda_{n+1} & =\sum_{k=0}^{n} 2^{4 k+1}\left|E_{2 k+2}\right| \lambda_{n-k} \quad(n \geq 0), \\
\varrho_{0} & =-1 \\
(n+1) \varrho_{n+1} & =-\sum_{k=0}^{n} 2^{4 k+1}\left|E_{2 k+2}\right| \varrho_{n-k} \quad(n \geq 0)
\end{aligned}
$$

und

$$
\mu_{n}=\frac{\lambda_{n}+\varrho_{n}}{2} \quad(n \geq 1)
$$


gegeben. Dabei sind die $E_{2 k}$ die Eulerschen Zahlen.

Für $n \leq 6$ geben wir die Werte der Zahlen $\lambda_{n}$, $\varrho_{n}{ }^{6)}$ und $\mu_{n}$ in Form einer Primfaktorzerlegung an:

$$
\begin{array}{lll}
\lambda_{1}=2, & \lambda_{0}=1, & \\
\lambda_{2}=2 \cdot 41, & & \lambda_{4}=2 \cdot 3 \cdot 7 \cdot 68111, \\
\lambda_{3}=2^{2} \cdot 3 \cdot 881, & \lambda_{5}=2^{2} \cdot 3 \cdot 47 \cdot 499 \cdot 4729, \\
& & \\
\lambda_{6}=2^{2} \cdot 3 \cdot 409 \cdot 193077047, \\
\varrho_{1}=2, & \varrho_{0}=-1, & \\
\varrho_{2}=2 \cdot 3 \cdot 13, & \varrho_{4}=2 \cdot 313 \cdot 4493, \\
\varrho_{3}=2^{2} \cdot 11 \cdot 233, & \varrho_{5}=2^{2} \cdot 3 \cdot 37 \cdot 2968241, \\
\mu_{1}=2, & \varrho_{6}=2^{2} \cdot 61 \cdot 3859681871, \\
\mu_{2}=2^{4} \cdot 5, & & \\
\mu_{3}=2^{2} \cdot 19 \cdot 137, & \mu_{4}=2^{5} \cdot 5 \cdot 17729, \\
& & \mu_{5}=2^{2} \cdot 3 \cdot 17^{2} \cdot 521 \cdot 733, \\
& & \mu_{6}=2^{5} \cdot 5^{2} \cdot 179 \cdot 283 \cdot 23311 .
\end{array}
$$

Beweis. Wir können die einzelnen Schritte des Beweises hier nur andeuten, da dieser bei genauer Ausführung sehr umfangreich wird. Der Leser vergleiche dazu auch Siegel [32. S. 290 ff.], beachte jedoch, daß sich die hier auftretenden Integrale entsprechend unserer Herleitung der Riemann-Siegel-Formel aus der Riemann-Siegel-Integralformel von denen Siegels unterscheiden.

Wir setzen zur Abkürzung $\varepsilon:=e^{i \pi / 4}$ und erhalten mit der Parametrisierung $v=\varepsilon u(-\infty<u<+\infty)$ aus Satz 4.1 .2

$$
\widetilde{F}(q):=\frac{\varepsilon}{2 \sqrt{\pi}} e^{i \pi / 8-i q^{2} / 2} \int_{-\infty}^{+\infty} \frac{e^{-u^{2} / 2+q \varepsilon u}}{\cosh \frac{\sqrt{\pi}}{2} \varepsilon u} d u .
$$

Ersetzen wir $q$ mit $q+\varepsilon x$, so folgt

$$
2 \sqrt{\pi} \bar{\varepsilon} e^{-i \pi / 8+i(q+\varepsilon x)^{2} / 2} \widetilde{F}(q+\varepsilon x)=\int_{-\infty}^{+\infty} e^{i x u} \frac{e^{-u^{2} / 2+q \varepsilon u}}{\cosh \frac{\sqrt{\pi}}{2} \varepsilon u} d u
$$

und daraus mit $u=\bar{\varepsilon} v$ nach dem Satz von Fourier

$$
\frac{e^{i v^{2} / 2+q v}}{\cosh \frac{\sqrt{\pi}}{2} v}=\frac{\bar{\varepsilon}}{\sqrt{\pi}} e^{-i \pi / 8} \int_{-\infty}^{+\infty} e^{-\varepsilon v x} e^{i(q+\varepsilon x)^{2} / 2} \widetilde{F}(q+\varepsilon x) d x
$$

falls $v$ der Bedingung $|\Re(v)-\Im(v)|<\sqrt{\pi}$ genügt. Man erhält diese Bedingung, wenn man die für $\widetilde{F}(q+\varepsilon x)$ aus Satz 4.1 .2 folgende Darstellung

$$
\widetilde{F}(q+\varepsilon x)=\frac{e^{-i(q+\varepsilon x)^{2} / 2-i 3 \pi / 8}+i \sqrt{2} \cos \frac{\sqrt{\pi}}{2}(q+\varepsilon x)}{\cos \sqrt{\pi}(q+\varepsilon x)}
$$

6) In der Originalarbeit wurde auf die Angabe der $\varrho_{n}$ verzichtet. 
in den Integranden einsetzt und die Konvergenz des Integrals an der unteren und oberen Grenze untersucht.

In Satz 1.2.1, S. 12 machen wir die unwesentliche Einschränkung $a$ nicht ganz, so daß $-\sqrt{\pi}<q<\sqrt{\pi}$ wird, setzen in die dortige Integraldarstellung von $S$ die aus 4.16 folgende Gleichung

$$
e^{i \pi / 8-i q^{2} / 2} \frac{e^{i v^{2} / 2+q v}}{\cosh \frac{\sqrt{\pi}}{2} v}=\frac{\bar{\varepsilon}}{\sqrt{\pi}} \int_{-\infty}^{+\infty} e^{-x^{2} / 2-(v-i q) \varepsilon x} \widetilde{F}(q+\varepsilon x) d x
$$

ein und parametrisieren den Integrationsweg $\nearrow^{i q}$ mit $v=i q+\varepsilon \sqrt{2} u(-\infty<u<$ $+\infty)$. Die Bedingung $|\Re(v)-\Im(v)|<\sqrt{\pi}$ ist dann wegen $|\Re(v)-\Im(v)|=|q|$ erfüllt und es folgt

$$
S=\frac{1}{\sqrt{2} \pi} \int_{-\infty}^{+\infty} g(\tau, \varepsilon \sqrt{2} u)\left[\int_{-\infty}^{+\infty} e^{-x^{2} / 2-i \sqrt{2} u x} \widetilde{F}(q+\varepsilon x) d x\right] d u
$$

Wir tragen hier die Taylorreihe $\widetilde{F}(q+\varepsilon x)=\sum_{n=0}^{\infty} \widetilde{F}^{(n)}(q)(\varepsilon x)^{n} / n$ ! ein und erhalten nach Multiplikation mit

$$
U=\exp i\left(\frac{t}{2} \log \frac{t}{2 \pi}-\frac{t}{2}-\frac{\pi}{8}-\vartheta(t)\right)
$$

die formale Entwicklung

$$
U \cdot S \sim \sum_{n=0}^{\infty} \mathcal{D}_{n}^{*}(\tau) \frac{\widetilde{F}^{(n)}(q)}{n !}
$$

mit

$$
\mathcal{D}_{n}^{*}(\tau):=\frac{U \varepsilon^{n}}{\sqrt{2} \pi} \int_{-\infty}^{+\infty} g(\tau, \varepsilon \sqrt{2} u)\left[\int_{-\infty}^{+\infty} e^{-x^{2} / 2-i \sqrt{2} u x} x^{n} d x\right] d u \quad(n \geq 0) .
$$

Setzen wir für $n \geq 0$

$$
h_{n}(u):=\frac{i^{n}}{\sqrt{2 \pi}} 2^{n / 2} \int_{-\infty}^{+\infty} e^{-x^{2} / 2-i \sqrt{2} u x} x^{n} d x
$$

so wird $h_{n+1}(u)=-h_{n}^{\prime}(u)$. Wegen $h_{0}(u)=e^{-u^{2}}$ ist dann

$$
\left.h_{n}(u)=(-1)^{n} \frac{d^{n}}{d u^{n}}\left(e^{-u^{2}}\right)=e^{-u^{2}} H_{n}(u) 7\right)
$$

mit den Hermiteschen Polynomen $H_{n}(u)$. Wir erhalten so aus 4.18), wenn wir die Funktion $g(\tau, \varepsilon \sqrt{2} u)$ gleich mit ihrem expliziten Ausdruck aus Satz 1.2.1. S. 12 ersetzen und $\tau=1 /(2 \sqrt{2 t})$ beachten,

$$
\mathcal{D}_{n}^{*}(\tau)=\frac{\varepsilon^{-n} 2^{-n / 2}}{\sqrt{\pi}} U \int_{-\infty}^{+\infty} e^{(-1 / 2+i t) \log (1+i \varepsilon u / \sqrt{t})+\varepsilon \sqrt{t} u-u^{2} / 2} H_{n}(u) d u \quad(n \geq 0) .
$$

7) Siehe [1. 22.11.7]. 
Eine einfache Anwendung des Cauchyschen Integralsatzes zeigt, daß wir hier den Integrationsweg mit der durch den Verzweigungspunkt des Integranden bei $u=\varepsilon \sqrt{t}$ parallel zur reellen Achse verlaufenden Geraden ersetzen dürfen ${ }^{8)}$ Vir parametrisieren diesen Weg mit $u=\varepsilon \sqrt{t}+x(-\infty<x<+\infty)$ und erhalten nach Definition des Ausdruckes $U$ und der Funktion $\vartheta(t)$

$$
\mathcal{D}_{n}^{*}(\tau)=\frac{\varepsilon^{-n} 2^{-n / 2}}{\sqrt{\pi}} e^{\frac{1}{4} \log t+\frac{\pi}{4} t-i\left[\frac{t}{2} \log 2+\Im \log \Gamma\left(\frac{1}{4}+i \frac{t}{2}\right)\right]} W_{n}(t)
$$

mit

$$
W_{n}(t):=e^{-\pi t / 4-i \pi / 8} \int_{-\infty}^{+\infty} e^{-x^{2} / 2+(-1 / 2+i t) \log (i \varepsilon x)} H_{n}(\varepsilon \sqrt{t}+x) d x \quad(n \geq 0) .
$$

Zerlegt man dieses Integral in $\int_{0}^{\infty}+\int_{-\infty}^{0}$ und substituiert im zweiten Integral $x$ mit $-x$, so kann man das in die Form

$$
W_{n}(t)=\int_{0}^{\infty} e^{-x^{2} / 2} x^{-1 / 2+i t}\left[H_{n}(\varepsilon \sqrt{t}-x)-i e^{-\pi t} H_{n}(\varepsilon \sqrt{t}+x)\right] d x \quad(n \geq 0)
$$

bringen. Hieraus berechnet man wegen $H_{0}(x) \equiv 199$

$$
W_{0}(t)=\left(1-i e^{-\pi t}\right) 2^{-\frac{3}{4}+i \frac{t}{2}} \Gamma\left(\frac{1}{4}+i \frac{t}{2}\right)
$$

und wegen $H_{1}(x)=2 x{ }^{10)}$ ynter Verwendung des Ergänzungssatzes der Gammafunktion

$$
W_{1}(t)=2 \varepsilon \sqrt{t} W_{0}(t)-2^{\frac{3}{4}+i \frac{t}{2}} \frac{2 \pi \varepsilon e^{-\frac{\pi}{2} t}}{\Gamma\left(\frac{1}{4}+i \frac{t}{2}\right)} .
$$

Mit der Zerlegung

$$
\Gamma\left(\frac{1}{4}+i \frac{t}{2}\right)=e^{\Re \log \Gamma\left(\frac{1}{4}+i \frac{t}{2}\right)+i \Im \log \Gamma\left(\frac{1}{4}+i \frac{t}{2}\right)}
$$

folgt damit aus 4.19

$$
\begin{aligned}
& \mathcal{D}_{0}^{*}(\tau)=\left(1-i e^{-\pi t}\right) e^{\omega}, \\
& \mathcal{D}_{1}^{*}(\tau)=\sqrt{2 t}\left(\mathcal{D}_{0}^{*}(\tau)-e^{-\omega}\right),
\end{aligned}
$$

wobei wir zur Abkürzung

$$
\omega:=\Re \log \Gamma\left(\frac{1}{4}+i \frac{t}{2}\right)+\frac{\pi}{4} t+\frac{1}{4} \log \frac{t}{2}-\log \sqrt{2 \pi}
$$

8) Nach Definition der Funktion $g(\tau, z)$ in Satz 1.2.1, S. 12 ist der Logarithmus $\log (1+i \varepsilon u / \sqrt{t})$ im Integranden in der längs der Winkelhalbierenden des 1. Quadranten vom Punkt $\infty$ bis zum Punkt $u=\varepsilon \sqrt{t}$ aufgeschnittenen $u$-Ebene eine eindeutige holomorphe Funktion von $u$, die reelle Werte annimmt, wenn $1+i \varepsilon u / \sqrt{t}$ reell und positiv ist. Bei dieser Anwendung des Cauchyschen Integralsatzes wird der Schnitt in der $u$-Ebene daher nicht berührt.

9) Siehe [1. 22.4.8].

10) Siehe [1. 22.4.8]. 
gesetzt haben. Der letzte Ausdruck läßt sich für $t \rightarrow+\infty$ in eine asymptotische Reihe entwickeln. Wir verwenden die von Siegel in [32. S. 289, Nr. 43] angegebene Darstellung, aus der sich mit $\tau=1 /(2 \sqrt{2 t})$ die asymptotische Entwicklung

$$
\omega \sim \sum_{n=1}^{\infty} \frac{\left|E_{2 n}\right|}{n} 2^{4 n-3} \tau^{4 n} \quad(\tau \rightarrow 0)
$$

ergibt. Dabei sind die $E_{2 n}$ die Eulerschen Zahlen ${ }^{11)}$ Die Ausdrücke $e^{\omega}$ und $e^{-\omega}$ lassen sich daher in formale Potenzreihen der Form

$$
\begin{gathered}
e^{\omega} \sim \sum_{n=0}^{\infty} \lambda_{n} \tau^{4 n}, \\
e^{-\omega} \sim-\sum_{n=0}^{\infty} \varrho_{n} \tau^{4 n}
\end{gathered}
$$

entwickeln, in denen $\lambda_{0}=1$ und $\varrho_{0}=-1$ sind. Die Rekursionsformeln 4.13 und (4.14) ergeben sich dann in bekannter Weise durch Vergleich von 4.21) und (4.22). Für $t \rightarrow+\infty$ bzw. $\tau \rightarrow 0$ folgen damit aus (4.20) die asymptotischen Entwicklungen

$$
\begin{aligned}
& \mathcal{D}_{0}^{*}(\tau) \sim e^{\omega} \sim \sum_{n=0}^{\infty} \lambda_{n} \tau^{4 n}, \\
& \mathcal{D}_{1}^{*}(\tau) \sim \frac{e^{\omega}-e^{-\omega}}{2 \tau} \sim \sum_{n=1}^{\infty} \mu_{n} \tau^{4 n-1},
\end{aligned}
$$

wobei sich die Koeffizienten $\mu_{n}$ mit den Zahlen $\lambda_{n}$ und $\varrho_{n}$ nach Gleichung 4.15 errechnen.

Aus der Existenz einer Entwicklung der Form

$$
U \cdot S \sim \sum_{n=0}^{\infty} \mathcal{D}_{n}(\tau) \frac{\widetilde{F}^{(n)}(q)}{n !},
$$

in der die $\mathcal{D}_{n}(\tau)$ formale Potenzreihen in $\tau$ sind (Satz 2.1.4, S. 24), folgt durch Vergleich mit (4.17), daß die $\mathcal{D}_{n}(\tau)$ die formalen Potenzreihenentwicklungen der Funktionen $\mathcal{D}_{n}^{*}(\tau)$ sind. Aus 4.23 ergeben sich so für $\mathcal{D}_{0}(\tau)$ und $\mathcal{D}_{1}(\tau)$ die Gleichungen 4.11) und 4.12). Die angegebenen Werte der $\lambda_{n}$ und $\mu_{n}(n \leq 6)$ erhält man mit relativ geringem Rechenaufwand aus 4.13, 4.14 und 4.15. Damit ist der Beweis abgeschlossen.

\subsection{Verschiedene Hilfsabschätzungen}

Satz 4.4.1. Für reelle a mit $|a| \leq 1$ und beliebige reelle $x$ gelten die Abschätzungen

$$
\begin{aligned}
& \left|\frac{\cosh \left(e^{i \pi / 4} a x\right)}{\cosh \left(e^{i \pi / 4} x\right)}\right| \leq 1, \\
& \left|\frac{\sinh \left(e^{i \pi / 4} a x\right)}{\cosh \left(e^{i \pi / 4} x\right)}\right| \leq \sqrt{2} .
\end{aligned}
$$

11) Siegel bezeichnet $\left|E_{2 n}\right|$ mit $E_{n}$. 
Beweis. Bekanntlich ist für reelle $x$ und $y$

$$
\begin{aligned}
|\cosh (x+i y)|^{2} & =\frac{1}{2}(\cosh 2 x+\cos 2 y), \\
|\sinh (x+i y)|^{2} & =\frac{1}{2}(\cosh 2 x-\cos 2 y) .
\end{aligned}
$$

Aus 4.26 folgt mit $y=x$

$$
|\cosh (1+i) x|^{2}=\frac{1}{2}\left[\sum_{n=0}^{\infty} \frac{(2 x)^{2 n}}{(2 n) !}+\sum_{n=0}^{\infty}(-1)^{n} \frac{(2 x)^{2 n}}{(2 n) !}\right]=\sum_{n=0}^{\infty} \frac{(2 x)^{4 n}}{(4 n) !} .
$$

Für reelle $a$ mit $|a| \leq 1$ und beliebige reelle $x$ gilt dann die Abschätzung

$$
|\cosh (1+i) x|^{2}-|\cosh (1+i) a x|^{2}=\sum_{n=0}^{\infty}\left(1-a^{4 n}\right) \frac{(2 x)^{4 n}}{(4 n) !} \geq 0
$$

denn diese Reihe enthält nur Summanden $\geq 0$. Daher ist

$$
\left|\frac{\cosh (1+i) a x}{\cosh (1+i) x}\right| \leq 1
$$

und wegen $e^{i \pi / 4}=(1+i) / \sqrt{2}$ folgt hieraus die Abschätzung (4.24), wenn man $x$ mit $x / \sqrt{2}$ ersetzt.

Wiederum mit $y=x$ ist nach 4.26 und 4.27

$$
|\sinh (1+i) x|^{2}=\frac{1}{2}(\cosh 2 x+\cos 2 x)-\cos 2 x=|\cosh (1+i) x|^{2}-\cos 2 x,
$$

und wir erhalten unter Verwendung von 4.29

$$
\begin{aligned}
|\cosh (1+i) x|^{2}-|\sinh (1+i) a x|^{2} & =|\cosh (1+i) x|^{2}-|\cosh (1+i) a x|^{2}+\cos 2 a x \\
& \geq \cos 2 a x \geq-1 .
\end{aligned}
$$

Folglich gilt

$$
\left|\frac{\sinh (1+i) a x)}{\cosh (1+i) x}\right|^{2} \leq 1+\frac{1}{|\cosh (1+i) x|^{2}} \leq 2
$$

denn nach 4.28 ist $|\cosh (1+i) x|^{2} \geq 1$. Ersetzen wir wie eben $x$ mit $x / \sqrt{2}$, so ergibt sich die Abschätzung 4.25 und der Satz ist bewiesen.

Satz 4.4.2. Es sei x reell. Mit Hilfe der gewöhnlichen reellen Arkustangensfunktion gelten für die durch

$$
f(x):=\int_{0}^{x} v^{2} \frac{v-1}{1+(v-1)^{2}} d v
$$

definierte Funktion die Darstellungen

$$
\begin{array}{ll}
f(x)=\frac{x^{2}}{2}+x-2 \arctan (x-1)-\frac{\pi}{2} & \text { für alle reellen } x, \\
f(x)=\frac{x^{2}}{2}+x+2 \arctan \frac{x}{x-2} & \text { für } x<2 .
\end{array}
$$


$f(x)$ und $f(x)-f(1)$ genügen den Abschätzungen

$$
\begin{aligned}
& f(x)<\left\{\begin{array}{cc}
\frac{x^{2}}{2} & \text { für } x \leq-\theta, \\
\omega \frac{x^{2}}{2} & \text { für }-\theta<x<0,
\end{array}\right. \\
& f(x)-f(1)<0.56 x^{2} \quad \text { für } x \geq 1 .
\end{aligned}
$$

Dabei ist $\theta$ eine beliebige reelle $Z$ ahl $>0$ und

$$
\omega:=1-\frac{2}{\theta}+\frac{4}{\theta^{2}} \arctan \frac{\theta}{\theta+2} .
$$

Für diese $\theta$ ist $0<\omega<1$.

Beweis. Mit der Zerlegung

$$
v^{2} \frac{v-1}{1+(v-1)^{2}}=v+1-\frac{2}{1+(v-1)^{2}}
$$

folgt 4.31 unmittelbar aus 4.30. Mit Hilfe der Funktionalgleichung

$$
\arctan x=\arctan \frac{1+x}{1-x}-\frac{\pi}{4} \quad \text { für } x<1
$$

ergibt sich dann 4.32 aus 4.31.

Zur Herleitung der Abschätzung 4.33 sei jetzt $x<0$. Aus 4.32 folgt

$$
f(x)=\frac{x^{2}}{2} h(x)
$$

mit

$$
h(x):=1+\frac{2}{x}+\frac{4}{x^{2}} \arctan \frac{x}{x-2} .
$$

Für die erste Ableitung von $h(x)$ berechnet man

$$
h^{\prime}(x)=-\frac{2}{x^{2}}-\frac{8}{x^{3}} \arctan \frac{x}{x-2}-\frac{8}{x^{2}\left[x^{2}+(x-2)^{2}\right]} .
$$

Wegen $x<0$ gilt die Abschätzung

$$
-\frac{8}{x^{3}} \arctan \frac{x}{x-2}<-\frac{8}{x^{3}} \cdot \frac{x}{x-2}=-\frac{8}{x^{2}(x-2)},
$$

und wir erhalten

$$
h^{\prime}(x)<-\frac{2}{x^{2}}\left[1+\frac{4}{x-2}+\frac{4}{x^{2}+(x-2)^{2}}\right]=-\frac{4 x}{(x-2)\left[x^{2}+(x-2)^{2}\right]}<0,
$$

so daß $h(x)$ eine streng monoton fallende Funktion ist. Aus 4.30 folgt aber

$$
f^{\prime}(x)=x^{2} \frac{x-1}{1+(x-1)^{2}} .
$$


Daher ist $f^{\prime}(0)=f^{\prime \prime}(0)=0$ und damit

$$
f(x)=O\left(x^{3}\right) \quad \text { für } x \rightarrow 0 .
$$

Nach 4.36 ist dann

$$
h(x)=O(x) \quad \text { für } x \rightarrow 0,
$$

so daß $h(x)$ im Punkte $x=0$ mit dem Wert 0 stetig (sogar analytisch) ergänzbar ist. Wegen $h(-\infty)=1$ ist damit gezeigt, daß $h(x)$ monoton von 1 bis 0 fällt, wenn $x$ die reellen Zahlen von $-\infty$ bis 0 durchläuft. Wir können $h(x)$ daher mit

$$
h(x)<\left\{\begin{array}{cl}
1 & \text { für } x \leq-\theta, \\
h(-\theta) & \text { für }-\theta<x<0
\end{array}\right.
$$

abschätzen. Dabei ist $\theta$ eine beliebige reelle Zahl $>0$. Setzt man noch

$$
\omega:=h(-\theta)
$$

so ergibt sich hieraus zusammen mit 4.36 die Abschätzung 4.33). Die in 4.35 angegebene Darstellung von $\omega$ folgt aus (4.37) und wegen $0<h(x)<1$ für negative $x$ ist $0<\omega<1$ wie behauptet.

Es sei nun $x \geq 1$. Wir setzen

$$
w(x):=f(x)-f(1)-0.56 x^{2}
$$

und haben mit 4.38

$$
\begin{aligned}
w^{\prime}(x) & =x^{2} \frac{x-1}{1+(x-1)^{2}}-1.12 x \\
& =x \frac{-0.12 x^{2}+1.24 x-2.24}{1+(x-1)^{2}} \\
& =-0.12 x \frac{(x-7 / 3)(x-8)}{1+(x-1)^{2}} .
\end{aligned}
$$

Hieraus ergibt sich durch Untersuchung des Vorzeichens von $w^{\prime}(x)$, daß $w(x)$ für $1 \leq x \leq 7 / 3$ und $x \geq 8$ streng monoton fallend und für $7 / 3 \leq x \leq 8$ streng monoton steigend ist. Daher hat $w(x)$ bei $x=7 / 3$ ein lokales Minimum, bei $x=8$ ein lokales Maximum und genügt für alle $x \geq 1$ der Abschätzung

$$
w(x) \leq \max (w(1), w(8)) .
$$

Nach 4.39 ist aber

$$
w(1)=-0.56<0
$$

und unter Verwendung der Abschätzungen

$$
\begin{aligned}
f(8) & =40-2 \arctan 7-\frac{\pi}{2}=40-\frac{3 \pi}{2}+2 \arctan \frac{1}{7} \\
& <40-\frac{3 \pi}{2}+\frac{2}{7}<35.6,
\end{aligned}
$$




$$
-f(1)=-\frac{3-\pi}{2}<0.08,
$$

die sich aus 4.31 ergeben,

$$
w(8)<35.6+0.08-0.56 \cdot 64=-0.16<0,
$$

so daß $w(x)$ für alle $x \geq 1$ negativ ist. Die Abschätzung 4.34 folgt damit aus (4.39) und der Satz ist bewiesen.

Satz 4.4.3. Die unvollständige Gammafunktion ${ }^{12)}$

$$
\Gamma(a, x):=\int_{x}^{\infty} e^{-v} v^{a-1} d v \quad(\text { a beliebig reell, } x \text { reell }>0)
$$

läßt sich für $a \geq 1$ und $x>a$ mit

$$
\Gamma(a, x) \leq a e^{-x} x^{a-1}
$$

abschätzen.

Beweis. Zunächst sei $a \geq 0$. Mit der Substitution $v=1 / w$ folgt aus 4.40

$$
\Gamma(a, x)=\int_{0}^{1 / x} e^{-1 / w} w^{-a-1} d w .
$$

Setzen wir

$$
h(w):=e^{-1 / w} w^{-a-1},
$$

so wird die erste Ableitung dieser Funktion,

$$
h^{\prime}(w)=e^{-1 / w} w^{-a-2}\left[\frac{1}{w}-(a+1)\right],
$$

für alle $w$ aus dem Integrationsintervall von 4.41 positiv, wenn $x$ der Bedingung $x>a+1$ genügt. Daher ist $h(w)$ für diese $w$ und $x$ eine streng monoton wachsende Funktion von $w$, und wir erhalten aus 4.41 die Abschätzung

$$
\Gamma(a, x) \leq \frac{1}{x} h\left(\frac{1}{x}\right)=e^{-x} x^{a} \quad(a \geq 0, x>a+1) .
$$

Für $a \geq 1$ läßt sich diese Abschätzung noch verschärfen. Partielle Integration in (4.40) führt zu der Funktionalgleichung

$$
\Gamma(a, x)=e^{-x} x^{a-1}+(a-1) \Gamma(a-1, x) .
$$

Wendet man hier 4.42 mit $a-1$ statt $a$ an, so folgt die Abschätzung

$$
\Gamma(a, x) \leq e^{-x} x^{a-1}+(a-1) e^{-x} x^{a-1}=a e^{-x} x^{a-1} \quad(a \geq 1, x>a),
$$

und das war zu zeigen.

12) Siehe [1. Kap. 6.5]. 


\section{Satz 4.4.4.}

(a) Die Koeffizienten $B_{n}(q)$ in der formalen Entwicklung $S \sim \sum_{n=0}^{\infty} B_{n}(q) \tau^{n}$ genügen für $|q| \leq \sqrt{\pi}$ den Abschätzunger ${ }^{13)}$

$$
\begin{array}{cc}
\left|B_{2 n}(q)\right| \leq \frac{2^{n+1 / 2}}{\pi} \sum_{k=0}^{2 n} \frac{2^{k} a_{k}^{(2 n)}(n+k-1) !}{(2 n+2 k) !} & (n \geq 1), \\
\left|B_{2 n+1}(q)\right| \leq \frac{1}{2^{n-1} \sqrt{\pi}} \sum_{k=0}^{2 n+1} \frac{a_{k}^{(2 n+1)}}{2^{k}(2 n+2 k+1)(n+k) !} & (n \geq 0) .
\end{array}
$$

(b) Für diese q ist

$$
\begin{array}{rlrl} 
& \left|B_{0}(q)\right| \leq 1, & & \\
\left|B_{1}(q)\right| & <1.6, & \left|B_{6}(q)\right| & <\quad 4209, \\
\left|B_{2}(q)\right| & <3.3, & \left|B_{7}(q)\right|< & <37784, \\
\left|B_{3}(q)\right| & <13.5, & \left|B_{8}(q)\right|<372500, \\
\left|B_{4}(q)\right|<76, & \left|B_{9}(q)\right|<3974961, \\
\left|B_{5}(q)\right|<526, & \left|B_{10}(q)\right|<45428942 .
\end{array}
$$

Beweis. Aus 1.26 und 1.27 folgt

$$
\left|B_{n}(q)\right| \leq \sum_{k=0}^{n} \frac{a_{k}^{(n)}}{(n+2 k) !}\left|b_{n+2 k}(q)\right| \quad(n \geq 0)
$$

mit

$$
b_{m}(q)=\frac{1}{2 \sqrt{\pi}} e^{i \pi / 8-i q^{2} / 2} \int_{\nearrow^{i q}} \frac{e^{i v^{2} / 2+q v}}{\cosh \frac{\sqrt{\pi}}{2} v}(v-i q)^{m} d v \quad(m \geq 0,-\sqrt{\pi}<q \leq \sqrt{\pi}) .
$$

Für $m \geq 1$ verschwindet $(v-i q)^{m}$ bei $v=i q$ von mindestens erster Ordnung, so daß der Integrand im Punkte $v=i q$ auch für $q=\sqrt{\pi}$ holomorph ist. Daher können wir wie beim Beweis von Satz 3.1.1. S. 41 vorgehen. Wie dort setzen wir $\varepsilon:=e^{i \pi / 4}$ und erhalten mit der Parametrisierung $v=i q+\varepsilon u(-\infty<u<+\infty)$

$$
b_{m}(q)=\frac{\varepsilon}{2 \sqrt{\pi}} e^{i \pi / 8} \int_{-\infty}^{+\infty} \frac{e^{-u^{2} / 2}}{\cosh \frac{\sqrt{\pi}}{2}(i q+\varepsilon u)}(\varepsilon u)^{m} d u \quad(m \geq 1,-\sqrt{\pi}<q \leq \sqrt{\pi}),
$$

woraus sich mit (vgl. Beweis von Satz 3.1.3. S. 48

$$
\frac{|u|}{\left|\cosh \frac{\sqrt{\pi}}{2}(i q+\varepsilon u)\right|} \leq \frac{|u|}{\sinh \frac{\sqrt{2 \pi}}{4}|u|} \leq \frac{4}{\sqrt{2 \pi}}
$$

13) In der Originalarbeit sind diese Abschätzungen unnötigerweise auf den Bereich $-\sqrt{\pi}<q \leq$ $+\sqrt{\pi}$ beschränkt worden. 
die von $q$ unabhängige Abschätzung

$$
\left|b_{m}(q)\right| \leq \frac{2 \sqrt{2}}{\pi} \int_{0}^{\infty} e^{-u^{2} / 2} u^{m-1} d u=\frac{2^{(m+1) / 2}}{\pi} \Gamma\left(\frac{m}{2}\right) \quad(m \geq 1)
$$

ergibt. Trennen wir die Fälle $m$ gerade und $m$ ungerade, so heißt das

$$
\begin{aligned}
\left|b_{2 m}(q)\right| & \leq \frac{2^{m+1 / 2}(m-1) !}{\pi} & & (m \geq 1), \\
\left|b_{2 m+1}(q)\right| & \leq \frac{(2 m) !}{\sqrt{\pi} 2^{m-1} m !} & & (m \geq 0) .
\end{aligned}
$$

Wegen $b_{m}(-q)=(-1)^{m} b_{m}(q)$ gelten diese Abschätzungen auch noch für $q=-\sqrt{\pi}$ und aus 4.43) folgt für $|q| \leq \sqrt{\pi}$

$$
\begin{aligned}
\left|B_{2 n}(q)\right| & \leq \sum_{k=0}^{2 n} \frac{a_{k}^{(2 n)}}{(2 n+2 k) !}\left|b_{2 n+2 k}(q)\right| \\
& \leq \frac{2^{n+1 / 2}}{\pi} \sum_{k=0}^{2 n} \frac{2^{k} a_{k}^{(2 n)}(n+k-1) !}{(2 n+2 k) !}
\end{aligned}
$$

und

$$
\begin{aligned}
\left|B_{2 n+1}(q)\right| & \leq \sum_{k=0}^{2 n+1} \frac{a_{k}^{(2 n+1)}}{(2 n+2 k+1) !}\left|b_{2 n+2 k+1}(q)\right| \\
& \leq \frac{1}{2^{n-1} \sqrt{\pi}} \sum_{k=0}^{2 n+1} \frac{a_{k}^{(2 n+1)}}{2^{k}(2 n+2 k+1)(n+k) !},
\end{aligned}
$$

wie in (a) behauptet.

Nach Satz 4.1 .2 ist $b_{0}(q)=\widetilde{F}(q)$. Wegen $a_{0}^{(0)}=1$ ist daher $\left|B_{0}(q)\right|=|\widetilde{F}(q)|$, und aus der Integraldarstellung von $\widetilde{F}(q)$ aus Satz 4.1 .3 folgt die Abschätzung

$$
\left|B_{0}(q)\right| \leq \sqrt{2} \int_{0}^{\infty} e^{-\pi v^{2} / 2}\left|\frac{\cosh (\sqrt{\pi} q \bar{\varepsilon} v)}{\cosh (\pi \bar{\varepsilon} v)}\right| d v \quad(|q| \leq \sqrt{\pi}) .
$$

Es ist aber für $|q| \leq \sqrt{\pi}$ nach Satz 4.4.1

$$
\left|\frac{\cosh (\sqrt{\pi} q \bar{\varepsilon} v)}{\cosh (\pi \bar{\varepsilon} v)}\right|=\left|\frac{\cosh \left(\varepsilon \frac{q}{\sqrt{\pi}} \pi v\right)}{\cosh (\varepsilon \pi v)}\right| \leq 1
$$

und daher wie in (b) behauptet

$$
\left|B_{0}(q)\right| \leq \sqrt{2} \int_{0}^{\infty} e^{-\pi v^{2} / 2} d v=1 .
$$

Mit den exakten Werten der Zahlen $a_{k}^{(n)}$ aus Tabelle I, S. 91 lassen sich die beiden Ausdrücke von (a) leicht berechnen. Auf diese Weise erhält man die in (b) angegebenen expliziten Abschätzungen von $B_{n}(q)$ für $1 \leq n \leq 10$. 


\section{Literaturverzeichnis}

[1] M. Abramowitz, I. A. Stegun, Handbook of Mathematical Functions, National Bureau of Standards, Applied Mathematics Series, 55, Tenth Printing, 1972

[2] J. Arias de Reyna, Dynamical Zeta Functions and Kummer Congruences, Acta Arithmetica 119 (1), 2005, 39-52, http://arxiv.org/PS_cache/math/ pdf/0309/0309190v1.pdf

[3] J. Arias de Reyna, High precision computation of Riemann's zeta function by the Riemann-Siegel formula, I, Math. of Comp. 80 (274), 2011, 9951009, http://www.ams.org/journals/mcom/2011-80-274/S0025-5718-20 10-02426-3/home.html

[4] H. Behnke, F. Sommer, Theorie der analytischen Funktionen einer komplexen Veränderlichen, Springer Verlag, Berlin, Heidelberg, New York, 1965

[5] M. V. Berry, J. P. Keating, A new asymptotic representation for $\zeta\left(\frac{1}{2}+i t\right)$ and quantum spectral determinants, Proc. Royal Soc. Lond. A 437, 1992, 151173, http://www.phy.bris.ac.uk/people/berry_mv/the_papers/Berry23 3.pdf

[6] M. V. Berry, The Riemann-Siegel expansion for the zeta function: high orders and remainders, Proc. Royal Soc. Lond. A 450, 1995, 439-462, http://www. phy.bris.ac.uk/people/berry_mv/the_papers/berry265.pdf

[7] M. V. Berry, J. P. Keating, The Riemann Zeros and Eigenvalue Asymptotics, SIAM Review 41 (2), 1999, 236-266, http://jpkeating.files.wordpress. com/2010/11/32.pdf

[8] J. M. Borwein, D. M. Bradley, R. E. Crandall, Computational strategies for the Riemann zeta function, Journal of Computational and Applied Mathematics 121, 2000, 247-296, http://people.reed.edu/ crandall/papers/atta ch01.pdf

[9] R. P. Brent, J. van de Lune, H. J. J. te Riele, D. T. Winter, On the Zeros of the Riemann Zeta Function in the Critical Strip. II, Math. of Comp. 39 (160), 1982, 681-688, http://oai.cwi.nl/oai/asset/10742/10742A.pdf

[10] R. P. Brent, J. van de Lune, H. J. J. te Riele, D. T. Winter, The first 200,000,001 zeros of Riemann's zeta function, Computational Methods in Number Theory, Math. Centrum, Amsterdam, 1982, 389-403, http://maths . anu.edu.au/ brent/pd/rpb081i.pdf 
[11] K. Chandrasekharan, Einführung in die analytische Zahlentheorie, Lecture Notes in Mathematics 29, 1966, Springer Verlag, Berlin, Heidelberg, New York

[12] K. Chandrasekharan, Introduction to Analytic Number Theory, Springer Verlag, Berlin, Heidelberg, New York, 1968

[13] R. Courant, D. Hilbert, Methoden der mathematischen Physik, Springer Verlag, Berlin, Heidelberg, New York, 4. Auflage, 1993

[14] R. Courant, D. Hilbert, Methods of Mathematical Physics, Vol. 1, John Wiley \& Sons, New York, 1989 und Wiley-VCH, Weinheim, 2004

[15] F. D. Crary, J. B. Rosser, High Precision Coefficients Related to the Zeta Function, MRC Technical Summary Report 1344, May 1975, Madison, Wisconsin, USA

[16] H. M. Edwards, Riemann's Zeta Function, Academic Press, New York, 1974

[17] W. Gabcke, Nueva deducción y cotas explicitas des los restos de la fórmula de Riemann-Siegel, Dissertation, Göttingen 1979, spanische Übersetzung von Juan Arias de Reyna, 2003

[18] X. Gourdon, P. Sebah, Numerical evaluation of the Riemann Zeta-function, http://numbers.computation.free.fr/Constants/Miscellaneous/ zetaevaluations.pdf. 2003

[19] X. Gourdon, The $10^{13}$ first zeros of the Riemann Zeta function, and zeros computation at very large height, http://numbers.computation.free.fr /Constants/Miscellaneous/zetazeros1e13-1e24.pdf, 2004

[20] C. B. Haselgrove, Royal Society Mathematical Tables 6, Cambridge 1960

[21] J. C. Lagarias, A. M. Odlyzko, Computing $\pi(x)$ : An Analytic Method, J. Algorithms 8, 1987, 173-191, http://www.dtc.umn.edu/ odlyzko/doc/arch/ analytic.pi.of.x.pdf

[22] D. H. Lehmer, Extended Computation of the Riemann zeta function, Mathematika 3, 1956, 102-108

[23] D. H. Lehmer, On the roots of the Riemann zeta function, Acta Mathematica 95, 1956, 291-297, http://www.kryakin.com/files/Acta_Mat_\%282_55\%29 /acta106_57/95/95_09.pdf

[24] J. van de Lune, H. J. J. te Riele, D. T. Winter, Rigorous High Speed Separation of Zeros of Riemann's Zeta Function, Report NW 113/81, Mathematical Centre, Amsterdam, Oktober 1981, http://oai.cwi.nl/oai/asset/8955/8 955A.pdf

[25] L. J. Mordell, The definite integral $\int_{-\infty}^{\infty} \frac{e^{a x^{2}+b x}}{e^{c x}+d} d x$ and the analytic theory of numbers, Acta Mathematica 61, 1933, 323-360, http://www.springerlink. com/content/j1474815272w103h/fulltext.pdf 
[26] N. Nielsen, Handbuch der Theorie der Gammafunktion, B. G. Teubner, Leipzig, 1906; Nachdruck (Faksimile): Chelsea Pub. Company, New York, 1965

[27] A. M. Odlyzko, On the Distribution of Spacings Between Zeros of the Zeta Function, Math. of Comp. 48 (174), 1987, 273-308, http://www.dtc.umn. edu/ odlyzko/doc/arch/zeta.zero.spacing.pdf

[28] A. M. Odlyzko, A. Schönhage, Fast Algorithms for Multiple Evaluations of the Riemann Zeta Function, Trans. Amer. Math. Soc. 309 (2), 1988, 797-809, http://www.dtc.umn.edu/ odlyzko/doc/arch/fast.zeta.eval.pdf

[29] H. J. J. te Riele, On the Sign of the Difference $\pi(x)-l i(x)$, Math. of Comp. 48 (177), 1987, 323-328, http://www.ams.org/journals/mcom/1987-48-17 7/S0025-5718-1987-0866118-6/S0025-5718-1987-0866118-6.pdf

[30] J. B. Rosser, J. M. Yohe, L. Schoenfeld, Rigorous computation and the zeros of the Riemann zeta function, Cong. Proc. Int. Federation Information Process., 1968, 70-76, Spartan, Washington, D. C. y Macmillan, New York, 1969

[31] C. L. Siegel, Über Riemanns Nachlaß zur analytischen Zahlentheorie, Quellenstudien zur Geschichte der Mathematik, Astronomie und Physik, Abt. B, Studien 2, 1932, 45-80

[32] C. L. Siegel, Gesammelte Abhandlungen 1, 275-310, Springer Verlag, Berlin, Heidelberg, New York, 1966

[33] E. C. Titchmarsh, The Zeros of the Riemann Zeta-Function, Proc. Royal Soc. Lond. A 151, 1935, 234-255, http://www.ift.uni.wroc.pl/ mwolf/Proc_ Roy_Soc_Lond_151\%281936\%29.pdf

[34] E. C. Titchmarsh, The Theory of the Riemann Zeta-Function, Oxford, 1951

[35] G. N. Watson, A Treatise on the Theory of the Bessel Functions, 2. Edition, Cambridge, 1966 



\section{Tabellen}

I. Primfaktorzerlegung der Zahlen $a_{k}^{(n)}$

$$
(0 \leq n \leq 11)
$$




\begin{tabular}{|c|c|c|r|r|r|r|}
$k \downarrow \vec{n}$ & 0 & 1 & \multicolumn{1}{c|}{2} & \multicolumn{1}{c|}{3} & \multicolumn{1}{c|}{4} \\
\hline 0 & 1 & 1 & 3 & $3 \cdot 5$ & $3 \cdot 5 \cdot 7$ & \multicolumn{1}{c|}{5} \\
1 & & 2 & $2^{2} \cdot 5$ & $2^{3} \cdot 3^{3}$ & $2^{2} \cdot 3 \cdot 223$ & $2 \cdot 3 \cdot 6323$ \\
2 & & & $2^{3} \cdot 5$ & $2^{5} \cdot 5 \cdot 7$ & $2^{4} \cdot 3 \cdot 7^{2} \cdot 11$ & $2^{4} \cdot 3^{4} \cdot 5 \cdot 7 \cdot 13$ \\
3 & & & & $2^{6} \cdot 5 \cdot 7$ & $2^{6} \cdot 5^{2} \cdot 7 \cdot 11$ & $2^{5} \cdot 3 \cdot 5 \cdot 7^{2} \cdot 11 \cdot 19$ \\
4 & & & & & $2^{7} \cdot 5^{2} \cdot 7 \cdot 11$ & $2^{7} \cdot 5^{2} \cdot 7^{2} \cdot 11 \cdot 13$ \\
5 & & & & & & $2^{8} \cdot 5^{2} \cdot 7^{2} \cdot 11 \cdot 13$ \\
\hline
\end{tabular}

\begin{tabular}{|c|r|r|}
$k \downarrow$ & \multicolumn{1}{|c|}{} & \multicolumn{1}{|c|}{7} \\
\hline 0 & $3^{3} \cdot 5 \cdot 7 \cdot 11$ & $3^{3} \cdot 5 \cdot 7 \cdot 11 \cdot 13$ \\
1 & $2^{3} \cdot 3^{2} \cdot 5 \cdot 19 \cdot 89$ & $2^{5} \cdot 3^{2} \cdot 5 \cdot 7591$ \\
2 & $2^{5} \cdot 3^{3} \cdot 127^{2}$ & $2^{4} \cdot 3^{4} \cdot 11 \cdot 24371$ \\
3 & $2^{6} \cdot 3 \cdot 5 \cdot 7 \cdot 11 \cdot 29 \cdot 83$ & $2^{6} \cdot 3 \cdot 11 \cdot 13 \cdot 17 \cdot 19 \cdot 709$ \\
4 & $2^{8} \cdot 3^{2} \cdot 5 \cdot 7^{2} \cdot 11 \cdot 13 \cdot 17$ & $2^{7} \cdot 3 \cdot 5^{2} \cdot 7^{2} \cdot 11 \cdot 13 \cdot 29 \cdot 37$ \\
5 & $2^{11} \cdot 5^{2} \cdot 7^{2} \cdot 11 \cdot 13 \cdot 17$ & $2^{11} \cdot 3 \cdot 5^{2} \cdot 7^{2} \cdot 11 \cdot 13 \cdot 17 \cdot 29$ \\
6 & $2^{12} \cdot 5^{2} \cdot 7^{2} \cdot 11 \cdot 13 \cdot 17$ & $2^{13} \cdot 5^{3} \cdot 7^{2} \cdot 11 \cdot 13 \cdot 17 \cdot 19$ \\
7 & & $2^{14} \cdot 5^{3} \cdot 7^{2} \cdot 11 \cdot 13 \cdot 17 \cdot 19$ \\
\hline
\end{tabular}

\begin{tabular}{|c|r|r|}
$k \downarrow \vec{n}$ & \multicolumn{1}{|c|}{9} \\
\hline 0 & $3^{4} \cdot 5^{2} \cdot 7 \cdot 11 \cdot 13$ & $3^{4} \cdot 5^{2} \cdot 7 \cdot 11 \cdot 13 \cdot 17$ \\
1 & $2^{3} \cdot 3^{2} \cdot 5 \cdot 173 \cdot 3491$ & $2 \cdot 3^{3} \cdot 5 \cdot 7 \cdot 2512297$ \\
2 & $2^{4} \cdot 3^{2} \cdot 11^{2} \cdot 527627$ & $2^{4} \cdot 3^{2} \cdot 5 \cdot 11 \cdot 13 \cdot 2511011$ \\
3 & $2^{9} \cdot 3^{4} \cdot 5 \cdot 7 \cdot 11 \cdot 13^{2} \cdot 83$ & $2^{5} \cdot 3^{2} \cdot 7^{3} \cdot 11 \cdot 13 \cdot 73 \cdot 89^{2}$ \\
4 & $2^{8} \cdot 3 \cdot 5 \cdot 7 \cdot 11 \cdot 13^{3} \cdot 5477$ & $2^{8} \cdot 3^{2} \cdot 5 \cdot 7 \cdot 11 \cdot 13^{2} \cdot 17 \cdot 47 \cdot 1429$ \\
5 & $2^{14} \cdot 3^{3} \cdot 5^{2} \cdot 7^{2} \cdot 11 \cdot 13 \cdot 17 \cdot 29$ & $2^{9} \cdot 3^{3} \cdot 5 \cdot 7 \cdot 11 \cdot 13 \cdot 17 \cdot 19^{2} \cdot 71 \cdot 83$ \\
6 & $2^{12} \cdot 3 \cdot 5^{2} \cdot 7^{2} \cdot 11 \cdot 13 \cdot 17^{2} \cdot 19 \cdot 23$ & $2^{12} \cdot 3 \cdot 5^{2} \cdot 7^{3} \cdot 11 \cdot 13 \cdot 17 \cdot 19 \cdot 5273$ \\
7 & $2^{14} \cdot 5^{3} \cdot 7^{2} \cdot 11^{2} \cdot 13 \cdot 17 \cdot 19 \cdot 23$ & $2^{13} \cdot 3^{2} \cdot 5^{2} \cdot 7^{2} \cdot 11^{2} \cdot 13^{3} \cdot 17 \cdot 19 \cdot 23$ \\
8 & $2^{15} \cdot 5^{3} \cdot 7^{2} \cdot 11^{2} \cdot 13 \cdot 17 \cdot 19 \cdot 23$ & $2^{15} \cdot 5^{5} \cdot 7^{2} \cdot 11^{2} \cdot 13^{2} \cdot 17 \cdot 19 \cdot 23$ \\
9 & & $2^{16} \cdot 5^{5} \cdot 7^{2} \cdot 11^{2} \cdot 13^{2} \cdot 17 \cdot 19 \cdot 23$ \\
\hline
\end{tabular}

\begin{tabular}{|r|r|}
$k \downarrow \vec{n}$ & 10 \\
\hline 0 & $3^{4} \cdot 5^{2} \cdot 7 \cdot 11 \cdot 13 \cdot 17 \cdot 19$ \\
1 & $2^{2} \cdot 3^{3} \cdot 5 \cdot 7 \cdot 29894203$ \\
2 & $2^{3} \cdot 3^{4} \cdot 5^{2} \cdot 13^{2} \cdot 2820197$ \\
3 & $2^{8} \cdot 3^{2} \cdot 7 \cdot 11 \cdot 13^{3} \cdot 37 \cdot 21323$ \\
4 & $2^{8} \cdot 3^{2} \cdot 7^{2} \cdot 11 \cdot 13 \cdot 17 \cdot 29923237$ \\
5 & $2^{10} \cdot 3^{5} \cdot 5^{2} \cdot 7^{2} \cdot 11 \cdot 13^{2} \cdot 17 \cdot 19 \cdot 853$ \\
6 & $2^{11} \cdot 3 \cdot 5^{3} \cdot 7^{2} \cdot 11^{2} \cdot 13 \cdot 17 \cdot 19 \cdot 281 \cdot 401$ \\
7 & $2^{15} \cdot 3 \cdot 5^{3} \cdot 7^{2} \cdot 11^{2} \cdot 13 \cdot 17 \cdot 19 \cdot 23 \cdot 3037$ \\
8 & $2^{15} \cdot 3 \cdot 5^{4} \cdot 7^{2} \cdot 11^{3} \cdot 13^{2} \cdot 17 \cdot 19 \cdot 23 \cdot 29$ \\
9 & $2^{17} \cdot 5^{5} \cdot 7^{3} \cdot 11^{2} \cdot 13^{2} \cdot 17 \cdot 19 \cdot 23 \cdot 29$ \\
10 & $2^{18} \cdot 5^{5} \cdot 7^{3} \cdot 11^{2} \cdot 13^{2} \cdot 17 \cdot 19 \cdot 23 \cdot 29$ \\
\hline
\end{tabular}




\begin{tabular}{|r|r|}
$k \downarrow \vec{n}$ & \multicolumn{1}{c|}{11} \\
\hline 0 & $3^{5} \cdot 5^{2} \cdot 7^{2} \cdot 11 \cdot 13 \cdot 17 \cdot 19$ \\
1 & $2^{3} \cdot 3^{3} \cdot 5^{2} \cdot 7 \cdot 2857 \cdot 26959$ \\
2 & $2^{4} \cdot 3^{3} \cdot 5 \cdot 13 \cdot 1571 \cdot 5542021$ \\
3 & $2^{7} \cdot 3^{3} \cdot 13^{2} \cdot 17 \cdot 34217 \cdot 35323$ \\
4 & $2^{8} \cdot 3^{2} \cdot 5^{2} \cdot 7 \cdot 11 \cdot 13 \cdot 17 \cdot 19 \cdot 37 \cdot 577531$ \\
5 & $2^{12} \cdot 3^{2} \cdot 5 \cdot 7^{2} \cdot 11 \cdot 13 \cdot 17 \cdot 19^{2} \cdot 1201327$ \\
6 & $2^{12} \cdot 3^{3} \cdot 5^{2} \cdot 7^{2} \cdot 11^{2} \cdot 13 \cdot 17 \cdot 19 \cdot 23 \cdot 106801$ \\
7 & $2^{14} \cdot 3 \cdot 5^{3} \cdot 7^{2} \cdot 11^{2} \cdot 13 \cdot 17 \cdot 19 \cdot 23 \cdot 43 \cdot 14783$ \\
8 & $2^{15} \cdot 3^{4} \cdot 5^{4} \cdot 7^{2} \cdot 11^{2} \cdot 13^{2} \cdot 17^{2} \cdot 19 \cdot 23 \cdot 103$ \\
9 & $2^{20} \cdot 3 \cdot 5^{4} \cdot 7^{5} \cdot 11^{2} \cdot 13^{2} \cdot 17 \cdot 19 \cdot 23 \cdot 29$ \\
10 & $2^{22} \cdot 5^{5} \cdot 7^{3} \cdot 11^{2} \cdot 13^{2} \cdot 17 \cdot 19 \cdot 23 \cdot 29 \cdot 31$ \\
11 & $2^{23} \cdot 5^{5} \cdot 7^{3} \cdot 11^{2} \cdot 13^{2} \cdot 17 \cdot 19 \cdot 23 \cdot 29 \cdot 31$ \\
\hline
\end{tabular}



II. Primfaktorzerlegung der Zahlen $d_{k}^{(n)}$

$$
(0 \leq n \leq 12)
$$




\begin{tabular}{|r|r|r|r|r|r|r|}
$k \downarrow \vec{n}$ & 0 & 1 & \multicolumn{1}{|c|}{2} & \multicolumn{1}{|c|}{3} & \multicolumn{1}{|c|}{4} \\
\hline 0 & 1 & 2 & $2^{3} \cdot 5$ & $2^{6} \cdot 5 \cdot 7$ & $2^{7} \cdot 5^{2} \cdot 7 \cdot 11$ & $2^{8} \cdot 5^{2} \cdot 7^{2} \cdot 11 \cdot 13$ \\
1 & & & 2 & $2^{6}$ & $2^{6} \cdot 7 \cdot 11$ & $2^{8} \cdot 5 \cdot 7^{2} \cdot 11$ \\
2 & & & & 2 & $2^{2} \cdot 19$ & $2^{3} \cdot 17 \cdot 53$ \\
3 & & & & & 2 & $2^{4} \cdot 5$ \\
\hline
\end{tabular}

\begin{tabular}{|cr|r|}
\multicolumn{1}{r|}{$k \downarrow \vec{n}$} & \multicolumn{1}{|c|}{6} & \multicolumn{1}{|c|}{7} \\
\hline 0 & $2^{12} \cdot 5^{2} \cdot 7^{2} \cdot 11 \cdot 13 \cdot 17$ & $2^{14} \cdot 5^{3} \cdot 7^{2} \cdot 11 \cdot 13 \cdot 17 \cdot 19$ \\
1 & $2^{8} \cdot 5 \cdot 7^{2} \cdot 11 \cdot 13 \cdot 17$ & $2^{14} \cdot 5^{2} \cdot 7^{2} \cdot 11 \cdot 13 \cdot 17$ \\
2 & $2^{6} \cdot 13 \cdot 1453$ & $2^{10} \cdot 11 \cdot 13 \cdot 2131$ \\
3 & $2^{3} \cdot 3 \cdot 367$ & $2^{8} \cdot 61 \cdot 109$ \\
4 & $2^{4} \cdot 5$ & $2^{3} \cdot 3 \cdot 11 \cdot 37$ \\
5 & & $2^{4} \cdot 5$ \\
\hline
\end{tabular}

\begin{tabular}{|cr|r|}
$k \downarrow \vec{n}$ & \multicolumn{1}{|c|}{8} \\
\hline 0 & $2^{15} \cdot 5^{3} \cdot 7^{2} \cdot 11^{2} \cdot 13 \cdot 17 \cdot 19 \cdot 23$ & $2^{16} \cdot 5^{5} \cdot 7^{2} \cdot 11^{2} \cdot 13^{2} \cdot 17 \cdot 19 \cdot 23$ \\
1 & $2^{14} \cdot 5^{2} \cdot 7^{2} \cdot 11 \cdot 13 \cdot 17 \cdot 19 \cdot 23$ & $2^{16} \cdot 5^{2} \cdot 7^{2} \cdot 11^{2} \cdot 13^{2} \cdot 17 \cdot 19 \cdot 23$ \\
2 & $2^{11} \cdot 5 \cdot 7 \cdot 11 \cdot 13 \cdot 19 \cdot 587$ & $2^{12} \cdot 5 \cdot 7 \cdot 11^{2} \cdot 13 \cdot 17 \cdot 19 \cdot 773$ \\
3 & $2^{9} \cdot 11 \cdot 88651$ & $2^{10} \cdot 3 \cdot 7 \cdot 11 \cdot 13 \cdot 53 \cdot 1259$ \\
4 & $2^{4} \cdot 5^{2} \cdot 5281$ & $2^{5} \cdot 19 \cdot 107 \cdot 10597$ \\
5 & $2^{3} \cdot 3 \cdot 7 \cdot 61$ & $2^{7} \cdot 5 \cdot 19 \cdot 199$ \\
6 & $2 \cdot 41$ & $2^{2} \cdot 19 \cdot 137$ \\
\hline
\end{tabular}

\begin{tabular}{|cr|r|}
$k \downarrow \vec{n}$ & \multicolumn{1}{|c|}{10} & \multicolumn{1}{|c|}{11} \\
\hline 0 & $2^{18} \cdot 5^{5} \cdot 7^{3} \cdot 11^{2} \cdot 13^{2} \cdot 17 \cdot 19 \cdot 23 \cdot 29$ & $2^{23} \cdot 5^{5} \cdot 7^{3} \cdot 11^{2} \cdot 13^{2} \cdot 17 \cdot 19 \cdot 23 \cdot 29 \cdot 31$ \\
1 & $2^{16} \cdot 5^{4} \cdot 7^{2} \cdot 11^{2} \cdot 13^{2} \cdot 17 \cdot 19 \cdot 23 \cdot 29$ & $2^{23} \cdot 5^{4} \cdot 7^{3} \cdot 11^{2} \cdot 13^{2} \cdot 17 \cdot 19 \cdot 23 \cdot 29$ \\
2 & $2^{14} \cdot 5^{3} \cdot 7^{2} \cdot 11^{2} \cdot 13 \cdot 17 \cdot 19^{2} \cdot 37$ & $2^{16} \cdot 5^{3} \cdot 7^{2} \cdot 11^{2} \cdot 13 \cdot 17^{2} \cdot 19 \cdot 23 \cdot 359$ \\
3 & $2^{12} \cdot 7^{2} \cdot 11 \cdot 13 \cdot 17 \cdot 61 \cdot 3767$ & $2^{17} \cdot 5 \cdot 7^{2} \cdot 11 \cdot 13 \cdot 17 \cdot 19 \cdot 52889$ \\
4 & $2^{7} \cdot 3^{4} \cdot 13^{2} \cdot 17 \cdot 10499$ & $2^{11} \cdot 13 \cdot 17 \cdot 661 \cdot 625631$ \\
5 & $2^{5} \cdot 19 \cdot 257 \cdot 5527$ & $2^{11} \cdot 3 \cdot 5 \cdot 149 \cdot 93229$ \\
6 & $2^{4} \cdot 5 \cdot 7 \cdot 13 \cdot 19^{2}$ & $2^{5} \cdot 19 \cdot 31 \cdot 53629$ \\
7 & $2^{2} \cdot 19 \cdot 137$ & $2^{9} \cdot 19 \cdot 283$ \\
8 & & $2^{2} \cdot 19 \cdot 137$ \\
\hline
\end{tabular}

\begin{tabular}{|r|r|}
$k \downarrow \vec{n}$ & \multicolumn{1}{c|}{12} \\
\hline 0 & $2^{24} \cdot 5^{6} \cdot 7^{4} \cdot 11^{2} \cdot 13^{2} \cdot 17^{2} \cdot 19 \cdot 23 \cdot 29 \cdot 31$ \\
1 & $2^{23} \cdot 5^{5} \cdot 7^{4} \cdot 11^{2} \cdot 13^{2} \cdot 17 \cdot 19 \cdot 23 \cdot 29 \cdot 31$ \\
2 & $2^{17} \cdot 5^{3} \cdot 7^{2} \cdot 11^{2} \cdot 13^{2} \cdot 17 \cdot 19 \cdot 23 \cdot 31 \cdot 7411$ \\
3 & $2^{16} \cdot 3^{2} \cdot 5 \cdot 7^{2} \cdot 11^{2} \cdot 13 \cdot 17 \cdot 19 \cdot 23 \cdot 40459$ \\
4 & $2^{12} \cdot 13 \cdot 17 \cdot 19 \cdot 23 \cdot 360131573$ \\
5 & $2^{11} \cdot 19 \cdot 23 \cdot 101 \cdot 3062053$ \\
6 & $2^{6} \cdot 5 \cdot 18919 \cdot 88853$ \\
7 & $2^{5} \cdot 5^{2} \cdot 11 \cdot 19 \cdot 6737$ \\
8 & $2^{3} \cdot 19 \cdot 18523$ \\
9 & $2^{2} \cdot 3 \cdot 881$ \\
\hline
\end{tabular}


III. Exakte Darstellung der Koeffizienten $C_{n}(z)$

$$
(0 \leq n \leq 12)
$$




$$
\begin{aligned}
& C_{0}(z)=F(z):=\frac{\cos \frac{\pi}{2}\left(z^{2}+\frac{3}{4}\right)}{\cos \pi z} \\
& C_{1}(z)=\frac{F^{(3)}(z)}{2^{2} \cdot 3 \pi^{2}} \\
& C_{2}(z)=\frac{F^{(6)}(z)}{2^{5} \cdot 3^{2} \pi^{4}}+\frac{F^{(2)}(z)}{2^{4} \pi^{2}} \\
& C_{3}(z)=\frac{F^{(9)}(z)}{2^{7} \cdot 3^{4} \pi^{6}}+\frac{F^{(5)}(z)}{2^{3} \cdot 3 \cdot 5 \pi^{4}}+\frac{F^{(1)}(z)}{2^{5} \pi^{2}} \\
& C_{4}(z)=\frac{F^{(12)}(z)}{2^{11} \cdot 3^{5} \pi^{8}}+\frac{11 F^{(8)}(z)}{2^{9} \cdot 3^{2} \cdot 5 \pi^{6}}+\frac{19 F^{(4)}(z)}{2^{9} \cdot 3 \pi^{4}}+\frac{F(z)}{2^{7} \pi^{2}} \\
& C_{5}(z)=\frac{F^{(15)}(z)}{2^{13} \cdot 3^{6} \cdot 5 \pi^{10}}+\frac{7 F^{(11)}(z)}{2^{10} \cdot 3^{4} \cdot 5 \pi^{8}}+\frac{17 \cdot 53 F^{(7)}(z)}{2^{11} \cdot 3^{2} \cdot 5 \cdot 7 \pi^{6}}+\frac{5 F^{(3)}(z)}{2^{7} \cdot 3 \pi^{4}} \\
& C_{6}(z)=\frac{F^{(18)}(z)}{2^{16} \cdot 3^{8} \cdot 5 \pi^{12}}+\frac{17 F^{(14)}(z)}{2^{15} \cdot 3^{5} \cdot 5 \pi^{10}}+\frac{13 \cdot 1453 F^{(10)}(z)}{2^{14} \cdot 3^{4} \cdot 5^{2} \cdot 7 \pi^{8}}+\frac{367 F^{(6)}(z)}{2^{13} \cdot 3 \cdot 5 \pi^{6}}+\frac{5 F^{(2)}(z)}{2^{9} \pi^{4}} \\
& C_{7}(z)=\frac{F^{(21)}(z)}{2^{18} \cdot 3^{9} \cdot 5 \cdot 7 \pi^{14}}+\frac{F^{(17)}(z)}{2^{15} \cdot 3^{6} \cdot 5 \pi^{12}}+\frac{2131 F^{(13)}(z)}{2^{14} \cdot 3^{5} \cdot 5^{2} \cdot 7 \pi^{10}}+\frac{61 \cdot 109 F^{(9)}(z)}{2^{13} \cdot 3^{4} \cdot 5 \cdot 7 \pi^{8}} \\
& +\frac{11 \cdot 37 F^{(5)}(z)}{2^{14} \cdot 5 \pi^{6}}+\frac{5 F^{(1)}(z)}{2^{10} \pi^{4}} \\
& C_{8}(z)=\frac{F^{(24)}(z)}{2^{23} \cdot 3^{10} \cdot 5 \cdot 7 \pi^{16}}+\frac{23 F^{(20)}(z)}{2^{20} \cdot 3^{8} \cdot 5^{2} \pi^{14}}+\frac{19 \cdot 587 F^{(16)}(z)}{2^{20} \cdot 3^{6} \cdot 5^{2} \cdot 7 \pi^{12}}+\frac{88651 F^{(12)}(z)}{2^{17} \cdot 3^{5} \cdot 5^{2} \cdot 7 \pi^{10}} \\
& +\frac{5 \cdot 5281 F^{(8)}(z)}{2^{19} \cdot 3^{2} \cdot 7 \pi^{8}}+\frac{7 \cdot 61 F^{(4)}(z)}{2^{16} \pi^{6}}+\frac{41 F(z)}{2^{15} \pi^{4}} \\
& C_{9}(z)=\frac{F^{(27)}(z)}{2^{25} \cdot 3^{13} \cdot 5 \cdot 7 \pi^{18}}+\frac{13 F^{(23)}(z)}{2^{21} \cdot 3^{9} \cdot 5^{2} \cdot 7 \pi^{16}}+\frac{11 \cdot 773 F^{(19)}(z)}{2^{22} \cdot 3^{8} \cdot 5^{2} \cdot 7 \pi^{14}} \\
& +\frac{53 \cdot 1259 F^{(15)}(z)}{2^{19} \cdot 3^{5} \cdot 5^{3} \cdot 7 \pi^{12}}+\frac{19 \cdot 107 \cdot 10597 F^{(11)}(z)}{2^{21} \cdot 3^{4} \cdot 5^{2} \cdot 7 \cdot 11 \pi^{10}}+\frac{19 \cdot 199 F^{(7)}(z)}{2^{15} \cdot 3^{2} \cdot 7 \pi^{8}} \\
& +\frac{19 \cdot 137 F^{(3)}(z)}{2^{17} \cdot 3 \pi^{6}} \\
& C_{10}(z)=\frac{F^{(30)}(z)}{2^{28} \cdot 3^{14} \cdot 5^{2} \cdot 7 \pi^{20}}+\frac{29 F^{(26)}(z)}{2^{27} \cdot 3^{10} \cdot 5^{2} \cdot 7 \pi^{18}}+\frac{19 \cdot 37 F^{(22)}(z)}{2^{25} \cdot 3^{9} \cdot 5 \cdot 7 \pi^{16}} \\
& +\frac{61 \cdot 3767 F^{(18)}(z)}{2^{24} \cdot 3^{8} \cdot 5^{3} \pi^{14}}+\frac{13 \cdot 17 \cdot 10499 F^{(14)}(z)}{2^{24} \cdot 3 \cdot 5^{2} \cdot 7^{2} \cdot 11 \pi^{12}}+\frac{19 \cdot 257 \cdot 5527 F^{(10)}(z)}{2^{23} \cdot 3^{4} \cdot 5^{2} \cdot 7 \pi^{10}} \\
& +\frac{7 \cdot 13 \cdot 19^{2} F^{(6)}(z)}{2^{20} \cdot 3^{2} \pi^{8}}+\frac{19 \cdot 137 F^{(2)}(z)}{2^{19} \pi^{6}}
\end{aligned}
$$




$$
\begin{aligned}
C_{11}(z)= & \frac{F^{(33)}(z)}{2^{30} \cdot 3^{15} \cdot 5^{2} \cdot 7 \cdot 11 \pi^{22}}+\frac{F^{(29)}(z)}{2^{24} \cdot 3^{13} \cdot 5^{2} \cdot 7 \pi^{20}}+\frac{17 \cdot 359 F^{(25)}(z)}{2^{28} \cdot 3^{10} \cdot 5^{3} \cdot 7 \pi^{18}} \\
& +\frac{52889 F^{(21)}(z)}{2^{23} \cdot 3^{9} \cdot 5^{3} \cdot 7 \pi^{16}}+\frac{661 \cdot 625631 F^{(17)}(z)}{2^{26} \cdot 3^{6} \cdot 5^{3} \cdot 7^{2} \cdot 11 \pi^{14}}+\frac{149 \cdot 93229 F^{(13)}(z)}{2^{21} \cdot 3^{4} \cdot 5 \cdot 7 \cdot 11 \cdot 13 \pi^{12}} \\
& +\frac{19 \cdot 31 \cdot 53629 F^{(9)}(z)}{2^{24} \cdot 3^{4} \cdot 5 \cdot 7 \pi^{10}}+\frac{19 \cdot 283 F^{(5)}(z)}{2^{16} \cdot 3 \cdot 5 \pi^{8}}+\frac{19 \cdot 137 F^{(1)}(z)}{2^{20} \pi^{6}} \\
C_{12}(z)= & \frac{F^{(36)}(z)}{2^{34} \cdot 3^{17} \cdot 5^{2} \cdot 7 \cdot 11 \pi^{24}}+\frac{F^{(32)}(z)}{2^{32} \cdot 3^{14} \cdot 5^{2} \pi^{22}}+\frac{31 \cdot 7411 F^{(28)}(z)}{2^{32} \cdot 3^{13} \cdot 5^{3} \cdot 7^{2} \pi^{20}} \\
& +\frac{40459 F^{(24)}(z)}{2^{30} \cdot 3^{8} \cdot 5^{3} \cdot 7 \pi^{18}}+\frac{23 \cdot 360131573 F^{(20)}(z)}{2^{30} \cdot 3^{8} \cdot 5^{4} \cdot 7^{2} \cdot 11 \pi^{16}} \\
& +\frac{19 \cdot 23 \cdot 101 \cdot 3062053 F^{(16)}(z)}{2^{28} \cdot 3^{6} \cdot 5^{3} \cdot 7^{2} \cdot 11 \cdot 13 \pi^{14}}+\frac{18919 \cdot 88853 F^{(12)}(z)}{2^{28} \cdot 3^{5} \cdot 5 \cdot 7 \cdot 11 \pi^{12}} \\
& +\frac{5 \cdot 11 \cdot 19 \cdot 6737 F^{(8)}(z)}{2^{26} \cdot 3^{2} \cdot 7 \pi^{10}}+\frac{19 \cdot 18523 F^{(4)}(z)}{2^{24} \cdot 3 \pi^{8}}+\frac{3 \cdot 881 F(z)}{2^{22} \pi^{6}}
\end{aligned}
$$



IV. Potenzreihen der Koeffizienten $C_{n}(z)$

$$
\begin{gathered}
50 \text { Dezimalstellen } \\
(0 \leq n \leq 10) \\
C_{2 n}(z)=\sum_{k=0}^{\infty} c_{2 k}^{(2 n)} z^{2 k} \\
C_{2 n+1}(z)=\sum_{k=0}^{\infty} c_{2 k+1}^{(2 n+1)} z^{2 k+1}
\end{gathered}
$$




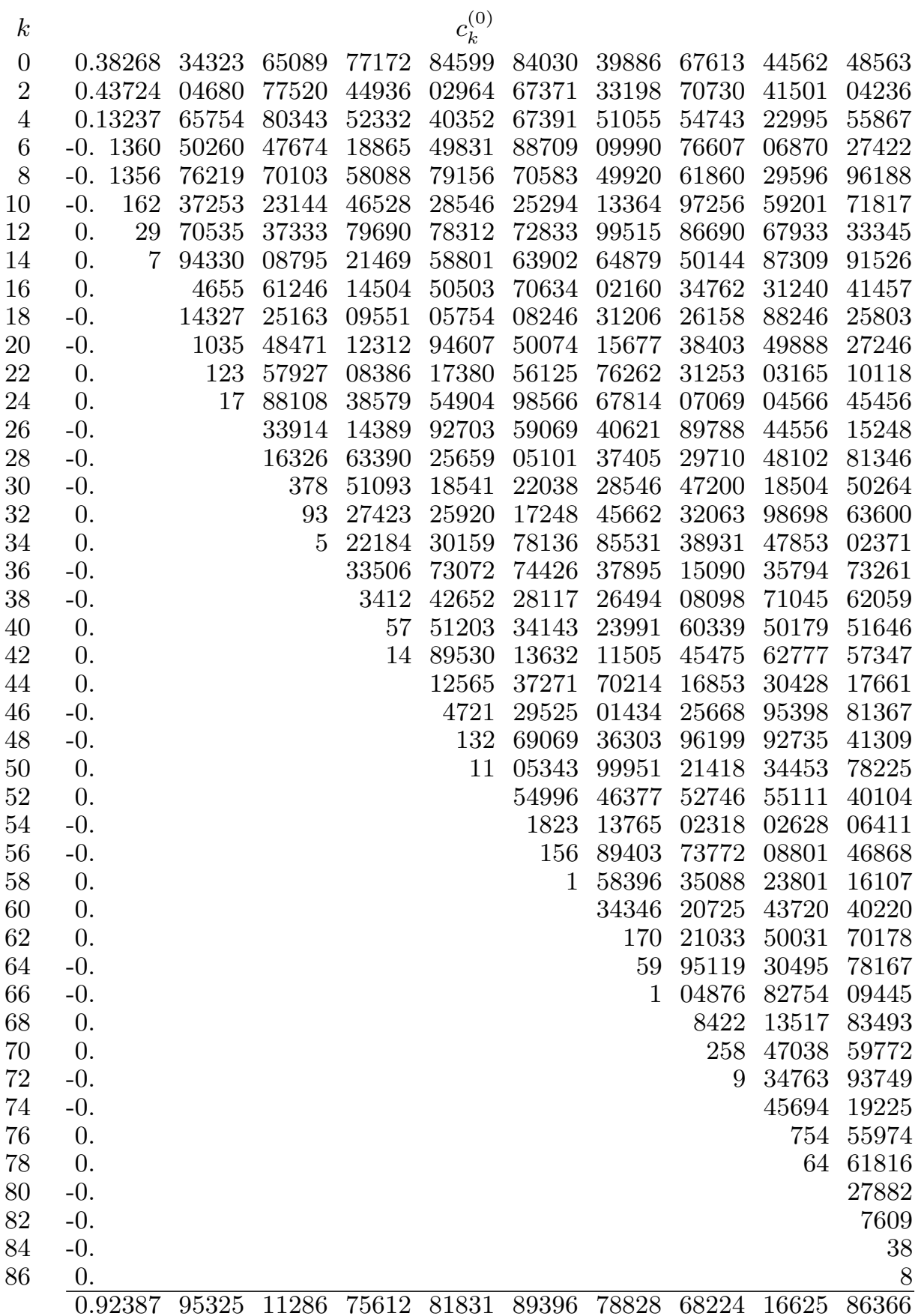




\begin{tabular}{|c|c|c|c|c|c|c|c|c|c|c|}
\hline$k$ & & & & & $c_{k}^{(1)}$ & & & & & \\
\hline 1 & 0.02682 & 51026 & 28375 & 34702 & 99914 & 03955 & 66674 & 96592 & 70472 & 43064 \\
\hline 3 & -0.1378 & 47734 & 26351 & 85304 & 98704 & 52589 & 89616 & 23659 & 48225 & 59753 \\
\hline 5 & -0. 3849 & 12504 & 82235 & 08222 & 87364 & 15363 & 18936 & 68960 & 98807 & 49451 \\
\hline 7 & $-0 . \quad 987$ & 10662 & 99062 & 07647 & 20121 & 47046 & 18854 & 06928 & 04214 & 59667 \\
\hline 9 & 0. 331 & 07597 & 60858 & 40433 & 29090 & 76951 & 30069 & 78028 & 02091 & 85612 \\
\hline 11 & 0. 146 & 47808 & 57795 & 41508 & 24977 & 96561 & 98311 & 19780 & 77545 & 77229 \\
\hline 13 & 0. & 32079 & 40624 & 87696 & 36751 & 61447 & 49443 & 09678 & 24291 & 83541 \\
\hline 15 & -0 & 92274 & 87018 & 47141 & 32322 & 34995 & 28189 & 56840 & 68029 & 12492 \\
\hline 17 & -0 & 59802 & 42585 & 37344 & 85877 & 10835 & 07451 & 58584 & 19335 & 89017 \\
\hline 19 & 0. & 9641 & 32245 & 61698 & 26352 & 67298 & 53298 & 51666 & 87570 & 78366 \\
\hline 21 & 0. & 1833 & 47337 & 22714 & 41176 & 00167 & 93657 & 83221 & 90807 & 53603 \\
\hline 23 & -0 & 44 & 67087 & 56271 & 78335 & 99560 & 79422 & 71505 & 51934 & 65747 \\
\hline 25 & -0 & 27 & 09635 & 08217 & 72743 & 21692 & 62839 & 87091 & 93725 & 93160 \\
\hline 27 & -0 & & 77852 & 88654 & 31585 & 10462 & 94823 & 08520 & 96100 & 06728 \\
\hline 29 & 0. & & 23437 & 62601 & 08936 & 88532 & 48455 & 04871 & 04512 & 27313 \\
\hline 31 & 0. & & 1583 & 01727 & 89987 & 52164 & 21622 & 26426 & 28742 & 11967 \\
\hline 33 & -0 & & 121 & 19941 & 57372 & 37912 & 46646 & 34473 & 80175 & 72576 \\
\hline 35 & -0 & & 14 & 58378 & 11611 & 08307 & 01758 & 28548 & 16989 & 99317 \\
\hline 37 & 0 . & & & 28786 & 30525 & 81319 & 17504 & 55821 & 28002 & 08761 \\
\hline 39 & 0 . & & & 8662 & 86290 & 21237 & 24122 & 52825 & 28879 & 33104 \\
\hline 41 & 0. & & & 84 & 30722 & 72713 & 70412 & 71560 & 02253 & 14627 \\
\hline 43 & -0 & & & 36 & 30807 & 22309 & 73462 & 00173 & 24618 & 11033 \\
\hline 45 & -0 & & & 1 & 16266 & 98212 & 83829 & 67194 & 13888 & 62925 \\
\hline 47 & 0. & & & & 10975 & 48671 & 15275 & 31815 & 90183 & 28340 \\
\hline 49 & 0. & & & & 615 & 73990 & 20468 & 42710 & 38814 & 70791 \\
\hline 51 & -0 & & & & 22 & 90928 & 00676 & 78471 & 51396 & 38263 \\
\hline 53 & -0 & & & & 2 & 20328 & 11748 & 84879 & 53437 & 95983 \\
\hline 55 & 0. & & & & & 2476 & 02518 & 00402 & 78508 & 28527 \\
\hline 57 & 0. & & & & & 595 & 42772 & 15583 & 65780 & 22727 \\
\hline 59 & 0 . & & & & & 3 & 26120 & 20746 & 79595 & 26153 \\
\hline 61 & -0 & & & & & 1 & 26540 & 35591 & 04116 & 22437 \\
\hline 63 & -0 & & & & & & 2431 & 28469 & 65496 & 98190 \\
\hline 65 & 0 . & & & & & & 213 & 83011 & 38754 & 69537 \\
\hline 67 & 0. & & & & & & 7 & 16779 & 94139 & 41062 \\
\hline 69 & -0 & & & & & & & 28242 & 93607 & 23367 \\
\hline 71 & -0 & & & & & & & 1500 & 60741 & 96069 \\
\hline 73 & 0 . & & & & & & & 26 & 87318 & 94053 \\
\hline 75 & 0 . & & & & & & & 2 & 49041 & 95008 \\
\hline 77 & -0 & & & & & & & & 1160 & 53898 \\
\hline 79 & -0 & & & & & & & & 341 & 37546 \\
\hline 81 & -0 & & & & & & & & 1 & 82473 \\
\hline 83 & 0. & & & & & & & & & 39328 \\
\hline 85 & 0. & & & & & & & & & 562 \\
\hline 87 & -0 & & & & & & & & & 38 \\
\hline 89 & -0 . & & & & & & & & & 1 \\
\hline
\end{tabular}




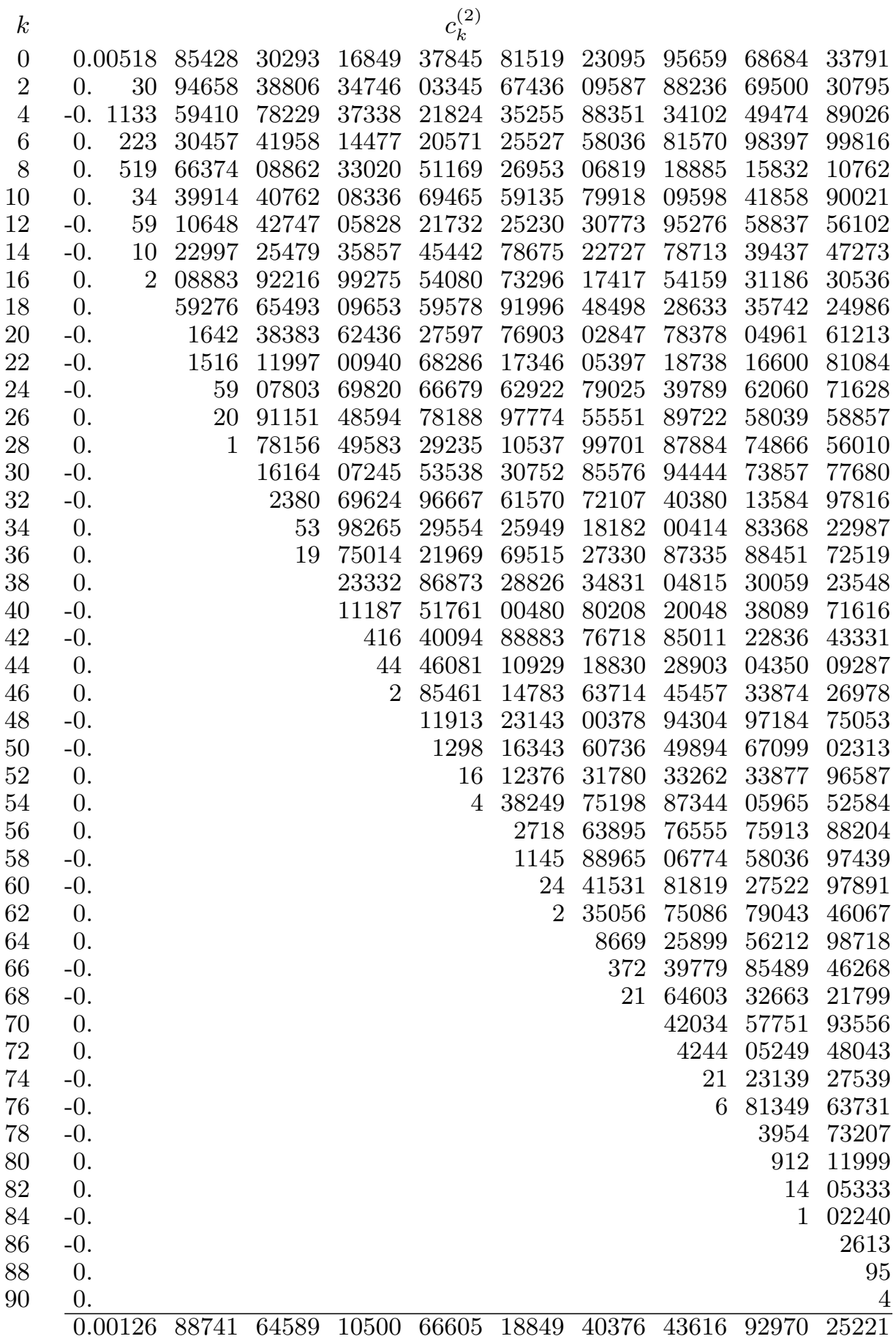




\begin{tabular}{|c|c|c|c|c|c|c|c|c|c|c|}
\hline$k$ & & & & & $c_{k}^{(3)}$ & & & & & \\
\hline 1 & 0.00133 & 97160 & 90719 & 45690 & 42698 & 35729 & 94522 & 81238 & 56353 & 95317 \\
\hline 3 & $-0 . \quad 374$ & 42151 & 36379 & 39370 & 46641 & 61864 & 46239 & 65812 & 84315 & 04245 \\
\hline 5 & 0. 133 & 03178 & 91932 & 14681 & 20318 & 54722 & 40241 & 05098 & 97088 & 24610 \\
\hline 7 & 0. 226 & 54660 & 76547 & 17871 & 14760 & 31990 & 52100 & 68874 & 11951 & 34489 \\
\hline 9 & $-0 . \quad 95$ & 48499 & 99850 & 67304 & 15112 & 25515 & 76501 & 13355 & 10463 & 76633 \\
\hline 11 & -0 & 10038 & 45896 & 36039 & 12075 & 80587 & 57956 & 11286 & 93255 & 59075 \\
\hline 13 & 10 & 12885 & 82867 & 76621 & 95334 & 43494 & 18087 & 85828 & 88131 & 81267 \\
\hline 15 & 0. & 86573 & 34492 & 99825 & 64245 & 74283 & 64865 & 21853 & 43285 & 92530 \\
\hline 17 & -0 . & 5985 & 36679 & 15385 & 98159 & 30593 & 38532 & 89474 & 47603 & 32543 \\
\hline 19 & -0 . & 33316 & 59851 & 23994 & 71290 & 43553 & 66983 & 83079 & 31712 & 85955 \\
\hline 21 & -0 & 2191 & 92891 & 02435 & 08105 & 71848 & 42192 & 25369 & 44570 & 56301 \\
\hline 23 & 0. & 789 & 08842 & 45681 & 49441 & 05552 & 48261 & 56888 & 52335 & 34195 \\
\hline 25 & 0. & 94 & 14685 & 08129 & 52621 & 51652 & 46515 & 67088 & 87214 & 34441 \\
\hline 27 & -0 & 9 & 57011 & 62108 & 83480 & 30188 & 07228 & 47736 & 89941 & 49204 \\
\hline 29 & -0 . & 1 & 87631 & 37453 & 47066 & 27968 & 12970 & 57776 & 33187 & 71497 \\
\hline 31 & 0. & & 4437 & 83767 & 93233 & 99327 & 46470 & 89849 & 67982 & 03943 \\
\hline 33 & 0. & & 2242 & 67385 & 05617 & 35324 & 84110 & 68573 & 06374 & 39088 \\
\hline 35 & 0. & & 36 & 27686 & 86573 & 52436 & 89408 & 25563 & 79232 & 00993 \\
\hline 37 & -0 & & 17 & 63980 & 95508 & 21581 & 60783 & 11214 & 98067 & 40561 \\
\hline 39 & -0 & & & 79607 & 65246 & 78677 & 77572 & 90345 & 17927 & 78777 \\
\hline 41 & 0. & & & 9419 & 65149 & 05896 & 90763 & 91489 & 50256 & 94424 \\
\hline 43 & 0. & & & 713 & 31038 & 54569 & 65782 & 45566 & 67924 & 63721 \\
\hline 45 & -0 & & & 32 & 89910 & 58455 & 46243 & 21179 & 66525 & 84927 \\
\hline 47 & -0 & & & 4 & 18073 & 03748 & 98459 & 29136 & 29248 & 70562 \\
\hline 49 & 0. & & & & 5550 & 54207 & 16463 & 33789 & 78211 & 64027 \\
\hline 51 & 0. & & & & 1787 & 04419 & 06260 & 12385 & 87176 & 36353 \\
\hline 53 & 0. & & & & 13 & 31280 & 39646 & 56094 & 28629 & 73430 \\
\hline 55 & -0 & & & & 5 & 81861 & 06110 & 90987 & 51617 & 92166 \\
\hline 57 & -0 & & & & & 14019 & 03608 & 85265 & 55374 & 36497 \\
\hline 59 & 0. & & & & & 1464 & 13202 & 11626 & 25414 & 89978 \\
\hline 61 & 0. & & & & & 60 & 23326 & 55108 & 91423 & 18945 \\
\hline 63 & -0 & & & & & 2 & 80644 & 72319 & 11360 & 74804 \\
\hline 65 & -0 & & & & & & 18065 & 06005 & 59245 & 48468 \\
\hline 67 & 0. & & & & & & 377 & 95083 & 31934 & 08111 \\
\hline 69 & 0. & & & & & & 42 & 14558 & 05294 & 75628 \\
\hline 71 & -0 & & & & & & & 22110 & 61928 & 33988 \\
\hline 73 & -0 & & & & & & & 7977 & 85719 & 14915 \\
\hline 75 & -0 & & & & & & & 51 & 34879 & 81542 \\
\hline 77 & 0. & & & & & & & 12 & 48640 & 63022 \\
\hline 79 & 0. & & & & & & & & 20921 & 85069 \\
\hline 81 & -0 & & & & & & & & 1623 & 63775 \\
\hline 83 & -0 & & & & & & & & 44 & 84110 \\
\hline 85 & 0. & & & & & & & & 1 & 73507 \\
\hline 87 & 0. & & & & & & & & & 7222 \\
\hline 89 & -0 & & & & & & & & & 146 \\
\hline 91 & -0 . & & & & & & & & & 10 \\
\hline & -0.00019 & 0002 & 59400 & 0243 & 222 & 94053 & 99643 & 43911 & 82834 & $\overline{46391}$ \\
\hline
\end{tabular}




\begin{tabular}{|c|c|c|c|c|c|c|c|c|c|c|}
\hline & & & & & $c_{k}^{(4)}$ & & & & & \\
\hline & 0.00046 & 48338 & 93617 & 63381 & 85363 & 04625 & 59567 & 24354 & 48586 & 069 \\
\hline & $-0 . \quad 100$ & 56607 & 36534 & 04707 & 59778 & 84972 & 86295 & 36576 & 07524 & 47568 \\
\hline & 0. & 04485 & 65737 & 25793 & 02244 & 56678 & 29485 & 74707 & 79638 & 6016 \\
\hline & 0. 102 & 83086 & 14970 & 23218 & 78262 & 98312 & 61578 & 75598 & 86311 & 79072 \\
\hline & -0 & 57861 & 07175 & 56441 & 86599 & 81580 & 00799 & 92688 & 20944 & 8499 \\
\hline & -0 & 36528 & 68030 & 84817 & 62148 & 43874 & 94623 & 41995 & 34626 & 9941 \\
\hline & 0. & 21229 & 04910 & 68727 & 89513 & 61265 & 01723 & 19707 & 47803 & \\
\hline & 0. & 26021 & 44243 & 86519 & 76077 & 37788 & 36663 & 42848 & 22539 & 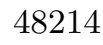 \\
\hline & -0 & 55790 & 62517 & 94952 & 51402 & 46040 & 07009 & 94523 & 16332 & 0306 \\
\hline & -0 & 41074 & 64438 & 91574 & 47539 & 81958 & 66 & 42973 & 65 & U. \\
\hline & 0. & 11781 & 11364 & 03712 & 93881 & 30076 & 93 & 24036 & & \\
\hline & 0. & 2445 & 65614 & 22484 & 57854 & 23157 & 09490 & 27874 & 00696 & 621 \\
\hline & -0 & 239 & 15824 & 6734 & 43224 & 30329 & & & & \\
\hline & -0 & 75 & 05214 & 20703 & 57552 & & & & & \\
\hline & 0. & 1 & 33122 & 79416 & 25842 & 81929 & 05 & & & . \\
\hline & 0 . & 1 & 34406 & 26754 & 22561 & 97186 & & & & . \\
\hline & 0 . & & 3513 & 77004 & 24304 & 85 & & & & 97 \\
\hline & -0 & & 1519 & 15445 & 33703 & & & & & 971 \\
\hline & -0 . & & 89 & 15417 & 68144 & 70 & & & & 297 \\
\hline & 0. & & 11 & 19589 & 11652 & 28 & & & & 580 \\
\hline & 0 . & & 1 & 05160 & 13329 & 91481 & & & & \\
\hline & -0 & & & 5178 & 65527 & & & & & 86 \\
\hline & -0 . & & & 806 & 58748 & 61 & & & & 2 \\
\hline & 0 . & & & 10 & 60820 & & & & & 9441 \\
\hline & 0 . & & & 4 & 43368 & 06742 & & & & 583 \\
\hline & 0. & & & & 4320 & 05 & & & & 0776 \\
\hline & -0 . & & & & 1823 & 03892 & & & & 22 \\
\hline & -0 & & & & 51 & 19936 & & & & \\
\hline & 0 . & & & & 5 & 69501 & 5 & & & 009 \\
\hline & 0 . & & & & & 26690 & & & & 274 \\
\hline & -0 . & & & & & 1333 & & & & 189 \\
\hline & -0 . & & & & & 96 & 85 & & & 1 \\
\hline & 0 . & & & & & 2 & & & & \\
\hline & 0. & & & & & & 27096 & & & 54 \\
\hline & -0 . & & & & & & 142 & & & 835 \\
\hline & -0 & & & & & & 60 & & & \\
\hline & -0 & & & & & & & 44916 & & 574 \\
\hline & 0 . & & & & & & & 11225 & & 246 \\
\hline & 0 & & & & & & & 207 & 66 & 3 \\
\hline & -0 & & & & & & & 17 & & 645 \\
\hline & -0 & & & & & & & & 51354 & 569 \\
\hline & 0 & & & & & & & & 2107 & 514 \\
\hline & U & & & & & & & & 95 & \\
\hline & -0 & & & & & & & & 2 & 035 \\
\hline & -0 & & & & & & & & & 143 \\
\hline & 0 . & & & & & & & & & 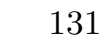 \\
\hline & P & & & & & & & & & \\
\hline
\end{tabular}




\begin{tabular}{|c|c|c|c|c|c|c|c|c|c|c|}
\hline$k$ & & & & & $c_{k}^{(5)}$ & & & & & \\
\hline 1 & -0.00011 & 34340 & 59228 & 68681 & 58827 & 89786 & 22736 & 30105 & 22768 & 18413 \\
\hline 3 & $\begin{array}{ll}-0 . & 13\end{array}$ & 85155 & 85671 & 47985 & 11219 & 83406 & 60473 & 59129 & 54479 & 06908 \\
\hline 5 & 0. & 68306 & 01735 & 94034 & 57526 & 51330 & 83952 & 90098 & 28198 & 76511 \\
\hline 7 & -0 & 22268 & 28546 & 77669 & 24003 & 22395 & 75379 & 70772 & 16031 & 22262 \\
\hline 9 & -0 & 02125 & 03923 & 89304 & 68611 & 33009 & 98232 & 85900 & 88004 & 62547 \\
\hline 11 & 0 . & 58333 & 02933 & 62498 & 66427 & 53866 & 44602 & 36078 & 73138 & 96532 \\
\hline 13 & -0 & 75048 & 68033 & 01063 & 88219 & 93319 & 07939 & 21787 & 84629 & 41549 \\
\hline 15 & -0 & 15691 & 32435 & 59332 & 80063 & 79547 & 01458 & 39659 & 32875 & 89769 \\
\hline 17 & 0. & 42177 & 25904 & 12201 & 97981 & 94005 & 98882 & 55782 & 08866 & 90245 \\
\hline 19 & 0. & 29158 & 99780 & 47906 & 36055 & 51463 & 54130 & 11298 & 76532 & 39676 \\
\hline 21 & -0 & 1565 & 37849 & 55844 & 68062 & 97453 & 25753 & 24030 & 83645 & 99484 \\
\hline 23 & -0 & 1465 & 21355 & 93176 & 92592 & 60207 & 41610 & 48857 & 87876 & 46533 \\
\hline 25 & -0 & 12 & 20015 & 86506 & 11429 & 90198 & 51976 & 36194 & 13186 & 65882 \\
\hline 27 & 0. & 41 & 61924 & 47590 & 97839 & 47671 & 64265 & 92800 & 11205 & 30751 \\
\hline 29 & 0. & 2 & 09398 & 12749 & 73436 & 33264 & 08788 & 16993 & 67138 & 15718 \\
\hline 31 & -0 & & 70839 & 55086 & 67248 & 85205 & 25367 & 99796 & 34999 & 37810 \\
\hline 33 & -0 & & 6023 & 00144 & 44422 & 42831 & 01924 & 87093 & 90509 & 30131 \\
\hline 35 & 0. & & 745 & 41536 & 44214 & 17556 & 59928 & 98761 & 90526 & 79952 \\
\hline 37 & 0 . & & 94 & 32313 & 17363 & 54683 & 70257 & 25080 & 65858 & 15943 \\
\hline 39 & -0 & & 4 & 63034 & 20856 & 22329 & 75611 & 90110 & 02523 & 48234 \\
\hline 41 & -0 & & & 96376 & 05904 & 06208 & 17943 & 39665 & 67623 & 56119 \\
\hline 43 & 0. & & & 1044 & 94028 & 45048 & 07270 & 93663 & 66465 & 72605 \\
\hline 45 & 0. & & & 693 & 87245 & 20141 & 27039 & 12210 & 28293 & 46941 \\
\hline 47 & 0. & & & 9 & 73300 & 20965 & 69540 & 47245 & 29578 & 55607 \\
\hline 49 & -0 & & & 3 & 66298 & 72527 & 67677 & 77079 & 46994 & 67508 \\
\hline 51 & -0 & & & & 12513 & 20911 & 93576 & 37011 & 34566 & 44686 \\
\hline 53 & 0 . & & & & 1440 & 44164 & 68986 & 79032 & 04974 & 33720 \\
\hline 55 & 0. & & & & 79 & 26953 & 53722 & 21720 & 69969 & 47117 \\
\hline 57 & -0 & & & & 4 & 16125 & 26928 & 83910 & 39027 & 55847 \\
\hline 59 & -0 & & & & & 35064 & 81184 & 11847 & 40816 & 69279 \\
\hline 61 & 0. & & & & & 807 & 72511 & 64436 & 28936 & 26811 \\
\hline 63 & 0. & & & & & 118 & 65496 & 28222 & 66413 & 63465 \\
\hline 65 & -0 & & & & & & 56844 & 10036 & 47862 & 51213 \\
\hline 67 & -0 & & & & & & 31957 & 27571 & 11389 & 34715 \\
\hline 69 & -0 & & & & & & 281 & 35861 & 65198 & 21126 \\
\hline 71 & 0. & & & & & & 69 & 83511 & 21565 & 27962 \\
\hline 73 & 0. & & & & & & 1 & 45059 & 53781 & 82417 \\
\hline 75 & -0 & & & & & & & 12452 & 92818 & 47399 \\
\hline 77 & -0 & & & & & & & 415 & 45871 & 96236 \\
\hline 79 & 0 & & & & & & & 17 & 93359 & 26609 \\
\hline 81 & 0. & & & & & & & & 89127 & 35667 \\
\hline 83 & -0 & & & & & & & & 1996 & 07660 \\
\hline 85 & -0 & & & & & & & & 155 & 02991 \\
\hline 87 & 0. & & & & & & & & 1 & 44958 \\
\hline 89 & 0. & & & & & & & & & 22618 \\
\hline 91 & 0. & & & & & & & & & 6 \\
\hline 93 & -0 & & & & & & & & & 28 \\
\hline & & & & & & & & & & \\
\hline
\end{tabular}




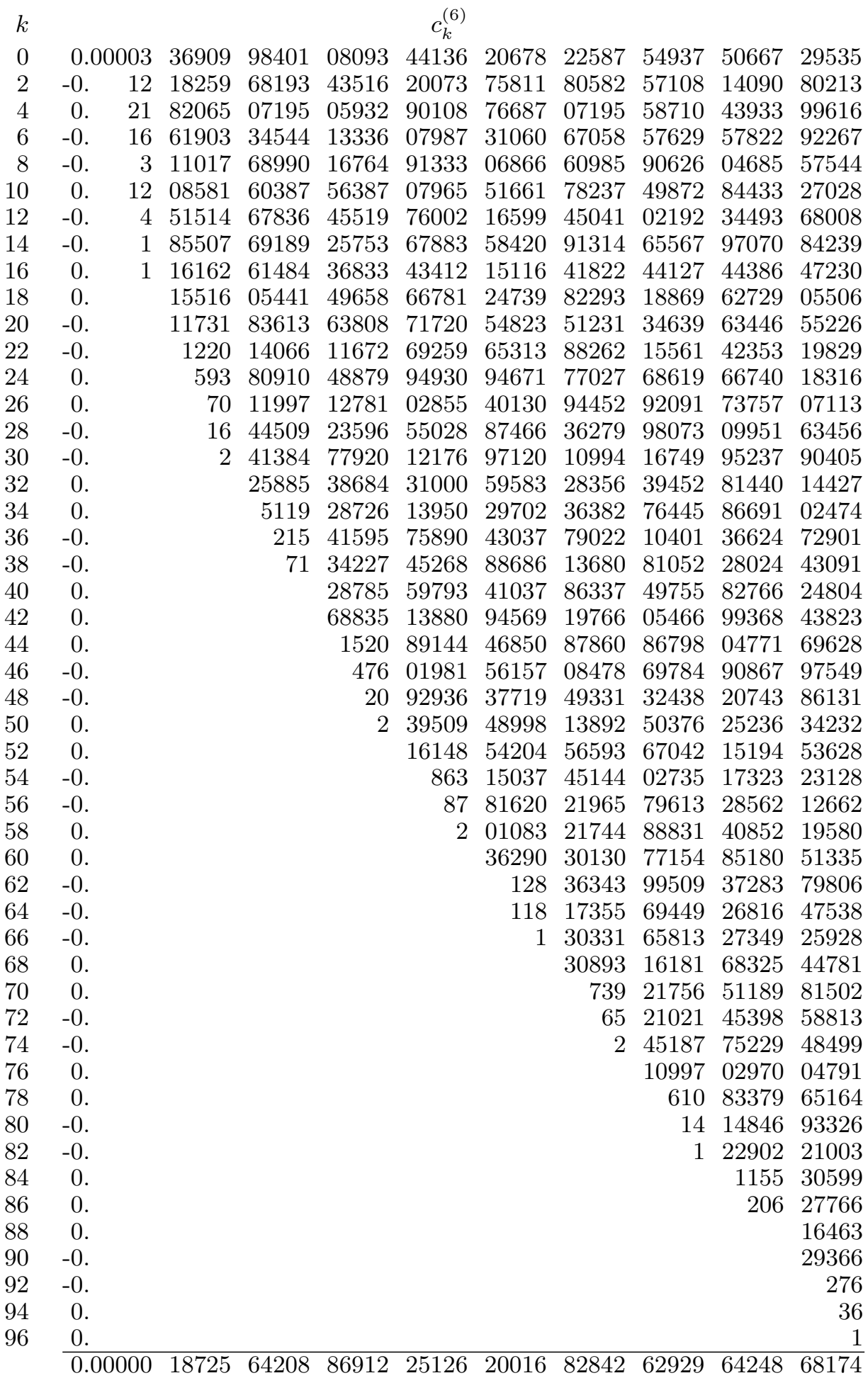




\begin{tabular}{|c|c|c|c|c|c|c|c|c|c|c|}
\hline$k$ & & & & & $c_{k}^{(7)}$ & & & & & \\
\hline 1 & -0.00003 & $\begin{array}{l}3 \quad 30623 \\
\end{array}$ & 99591 & 39952 & 29024 & 87318 & 67166 & 56535 & 46126 & 55816 \\
\hline 3 & 0. & $5 \quad 58380$ & 11971 & 67341 & 67188 & 25883 & 67807 & 70276 & 81352 & 19371 \\
\hline 5 & -0 & $\begin{array}{ll}3 & 38757\end{array}$ & 25212 & 77910 & 17489 & 14931 & 87425 & 42028 & 05779 & 11763 \\
\hline 7 & -0 & $\begin{array}{ll}392854 \\
\end{array}$ & 99154 & 42035 & 92030 & 93457 & 47011 & 47071 & 04055 & 24728 \\
\hline 9 & 0. & 759013 & 38897 & 08718 & 86441 & 75960 & 64660 & 85961 & 55637 & 56359 \\
\hline 11 & -0 & 376365 & 07526 & 41637 & 07541 & 13099 & 18865 & 92185 & 96404 & 10930 \\
\hline 13 & -0 & 77587 & 52128 & 98363 & 87786 & 77788 & 58804 & 21375 & 74674 & 71491 \\
\hline 15 & 0. & 124346 & 81009 & 03170 & 13854 & 62920 & 03156 & 97472 & 02285 & 22504 \\
\hline 17 & -0 & 13758 & 66097 & 40895 & 38622 & 58184 & 27541 & 54644 & 28908 & 06487 \\
\hline 19 & -0 & 15381 & 43632 & 05079 & 13348 & 05096 & 83866 & 74352 & 37943 & 86927 \\
\hline 21 & 0. & 2469 & 23352 & 16114 & 25500 & 28981 & 11363 & 41554 & 60802 & 12002 \\
\hline 23 & 0. & 1130 & 37488 & 41071 & 98166 & 87702 & 48571 & 03849 & 93083 & 27545 \\
\hline 25 & -0 & 140 & 65380 & 57682 & 03288 & 62357 & 68785 & 27784 & 22995 & 94048 \\
\hline 27 & -0 & 53 & 30220 & 99943 & 13446 & 47873 & 31522 & 07126 & 76247 & 57168 \\
\hline 29 & 0. & 3 & 59428 & 35262 & 85034 & 03526 & 62845 & 84527 & 72829 & 84714 \\
\hline 31 & 0. & 1 & 60914 & 68843 & 19826 & 04928 & 46417 & 33661 & 89535 & 13512 \\
\hline 33 & -0 & & 3405 & 39843 & 55486 & 28691 & 57518 & 47323 & 62007 & 67527 \\
\hline 35 & -0 & & 3175 & 02449 & 60695 & 34155 & 64297 & 79074 & 84549 & 67918 \\
\hline 37 & -0 & & 38 & 62417 & 13739 & 06374 & 27781 & 37102 & 34036 & 30784 \\
\hline 39 & 0. & & 42 & 37052 & 71361 & 14640 & 21794 & 42549 & 53403 & 62355 \\
\hline 41 & 0. & & 1 & 59919 & 33724 & 67790 & 45570 & 54455 & 23960 & 23808 \\
\hline 43 & -0 & & & 39390 & 48539 & 22384 & 81800 & 24475 & 70772 & 53182 \\
\hline 45 & -0 & & & 2400 & 16399 & 71157 & 29383 & 28357 & 36848 & 56489 \\
\hline 47 & 0. & & & 258 & 60510 & 85421 & 68898 & 16717 & 50238 & 85233 \\
\hline 49 & 0. & & & 22 & 70087 & 14433 & 08317 & 32323 & 82108 & 18990 \\
\hline 51 & -0 & & & 1 & 17488 & 73138 & 45793 & 16253 & 26962 & 16207 \\
\hline 53 & -0 & & & & 15334 & 67272 & 36759 & 85446 & 12061 & 66150 \\
\hline 55 & 0. & & & & 323 & 75436 & 58260 & 73211 & 61926 & 95556 \\
\hline 57 & 0. & & & & 78 & 27127 & 90820 & 78112 & 11950 & 09775 \\
\hline 59 & -0 & & & & & 7444 & 47157 & 21113 & 12244 & 32002 \\
\hline 61 & -0 & & & & & 31151 & 34644 & 40512 & 30502 & 01487 \\
\hline 63 & -0 & & & & & 452 & 18515 & 97017 & 93803 & 81976 \\
\hline 65 & 0. & & & & & 98 & 37527 & 95687 & 74038 & 90560 \\
\hline 67 & 0. & & & & & 2 & 79509 & 19701 & 42493 & 76037 \\
\hline 69 & -0 & & & & & & 24785 & 25849 & 80959 & 63219 \\
\hline 71 & -0 & & & & & & 1075 & 12538 & 89274 & 85989 \\
\hline 73 & 0. & & & & & & 49 & 25301 & 24764 & 37445 \\
\hline 75 & 0. & & & & & & 3 & 12461 & 76734 & 30532 \\
\hline 77 & -0 & & & & & & & 7341 & 99880 & 33856 \\
\hline 79 & -0 & & & & & & & 731 & 13688 & 37108 \\
\hline 81 & 0. & & & & & & & 6 & 65251 & 03881 \\
\hline 83 & 0. & & & & & & & 1 & 41940 & 11191 \\
\hline 85 & 0. & & & & & & & & 234 & 87748 \\
\hline 87 & -0 & & & & & & & & 232 & 32621 \\
\hline 89 & -0 & & & & & & & & 2 & 50026 \\
\hline 91 & 0. & & & & & & & & & 32307 \\
\hline 93 & 0. & & & & & & & & & 631 \\
\hline 95 & -0 . & & & & & & & & & 38 \\
\hline 97 & 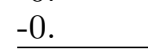 & & & & & & & & & 0 \\
\hline
\end{tabular}




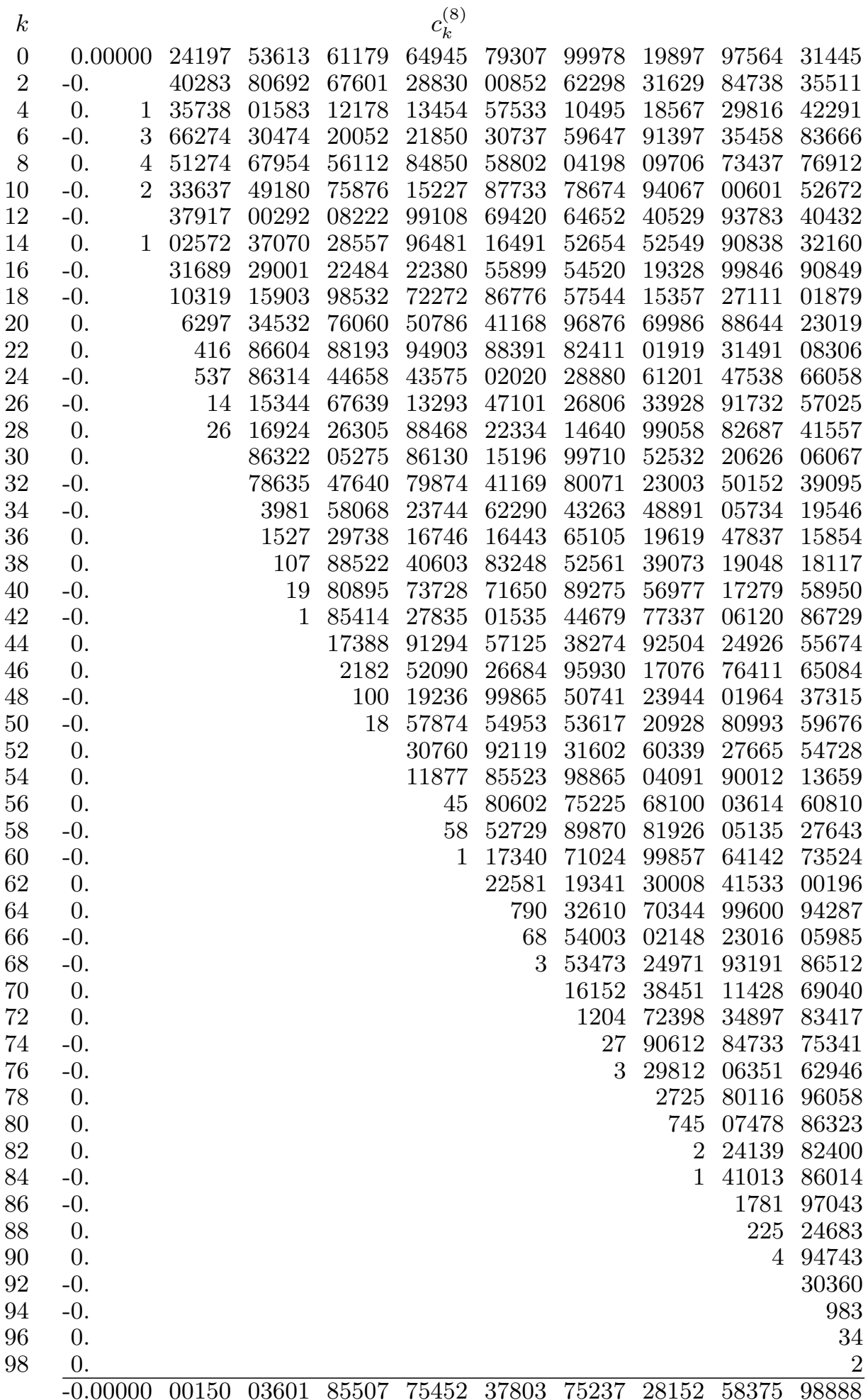




\begin{tabular}{|c|c|c|c|c|c|c|c|c|c|c|}
\hline$k$ & & & & & $c_{k}^{(9)}$ & & & & & \\
\hline 1 & -0.00000 & 68841 & 20503 & 02734 & 50464 & 61678 & 38197 & 25811 & 99444 & 30684 \\
\hline 3 & 0 . & 35455 & 33780 & 52358 & 40787 & 11064 & 18706 & 61993 & 04913 & 93998 \\
\hline 5 & -0 & 17540 & 23152 & 21147 & 69281 & 22088 & 64469 & 58296 & 52636 & 31740 \\
\hline 7 & 0. & 19947 & 55165 & 85995 & 83028 & 26621 & 51126 & 15048 & 55781 & 23332 \\
\hline 9 & -0 & 01782 & 84429 & 93048 & 87269 & 38862 & 25127 & 55859 & 00385 & 46125 \\
\hline 11 & -0 & 38363 & 10144 & 42745 & 01643 & 50318 & 98381 & 94483 & 19671 & 11373 \\
\hline 13 & 0. & 79196 & 29169 & 06357 & 19271 & 87869 & 34310 & 43663 & 71644 & 68478 \\
\hline 15 & -0 & 35151 & 96700 & 43216 & 13500 & 40118 & 19412 & 94289 & 51863 & 87945 \\
\hline 17 & -0 & 3734 & 79889 & 72624 & 76532 & 02202 & 63481 & 23077 & 27441 & 67754 \\
\hline 19 & 0. & 7600 & 05862 & 35439 & 53354 & 87465 & 83487 & 90019 & 84056 & 40210 \\
\hline 21 & -0 & 1139 & 08784 & 42965 & 69870 & 82245 & 27118 & 61653 & 79820 & 57387 \\
\hline 23 & -0 & 661 & 43447 & 45943 & 31655 & 01067 & 71236 & 30928 & 36143 & 71583 \\
\hline 25 & 0 . & 155 & 40047 & 96612 & 31488 & 53865 & 93748 & 08616 & 32114 & 81422 \\
\hline 27 & 0 . & 36 & 23234 & 09330 & 98040 & 42098 & 51629 & 23181 & 46055 & 15384 \\
\hline 29 & -0 & 8 & 81317 & 55262 & 99377 & 56404 & 41223 & 74833 & 59498 & 71733 \\
\hline 31 & -0 & 1 & 46709 & 98190 & 01162 & 69847 & 33220 & 09222 & 93424 & 19314 \\
\hline 33 & 0. & & 27908 & 71227 & 12363 & 27429 & 05112 & 28592 & 63778 & 66500 \\
\hline 35 & 0. & & 4368 & 63944 & 60535 & 95677 & 35802 & 80954 & 81247 & 14459 \\
\hline 37 & -0 & & 539 & 73899 & 08013 & 26311 & 59128 & 57526 & 07726 & 63407 \\
\hline 39 & -0 & & 92 & 62633 & 46009 & 83158 & 46339 & 76752 & 53933 & 72800 \\
\hline 41 & 0. & & 6 & 54313 & 49032 & 22681 & 55095 & 04111 & 34893 & 30789 \\
\hline 43 & 0. & & 1 & 39808 & 29940 & 39760 & 03943 & 34567 & 37704 & 20690 \\
\hline 45 & -0 & & & 4684 & 25717 & 71758 & 32438 & 53960 & 67365 & 02875 \\
\hline 47 & -0 & & & 1532 & 69898 & 48153 & 13897 & 59760 & 93775 & 81291 \\
\hline 49 & 0. & & & 11 & 17499 & 01875 & 44543 & 93309 & 39186 & 20007 \\
\hline 51 & 0. & & & 12 & 50412 & 07183 & 83387 & 47334 & 53760 & 93297 \\
\hline 53 & 0. & & & & 15591 & 11027 & 51327 & 96855 & 08452 & 84653 \\
\hline 55 & -0 & & & & 7751 & 97555 & 47983 & 23627 & 10236 & 56399 \\
\hline 57 & -0 & & & & 225 & 06761 & 63134 & 86007 & 04767 & 54281 \\
\hline 59 & 0. & & & & 37 & 03799 & 08075 & 91632 & 07533 & 13189 \\
\hline 61 & 0. & & & & 1 & 66976 & 01620 & 56056 & 73990 & 28104 \\
\hline 63 & -0 & & & & & 13686 & 12196 & 76531 & 54908 & 64469 \\
\hline 65 & -0 & & & & & 872 & 90672 & 85976 & 21409 & 35864 \\
\hline 67 & 0. & & & & & 38 & 47825 & 20643 & 39629 & 49644 \\
\hline 69 & 0. & & & & & 3 & 51172 & 41137 & 34726 & 79520 \\
\hline 71 & -0 & & & & & & 7669 & 68065 & 13064 & 04803 \\
\hline 73 & -0 & & & & & & 1132 & 65635 & 85874 & 94906 \\
\hline 75 & 0. & & & & & & 7 & 51939 & 04655 & 97091 \\
\hline 77 & 0. & & & & & & 2 & 99788 & 94515 & 91468 \\
\hline 79 & 0. & & & & & & & 1524 & 92924 & 80856 \\
\hline 81 & -0 & & & & & & & 660 & 24315 & 73341 \\
\hline 83 & -0 & & & & & & & 10 & 06295 & 90014 \\
\hline 85 & 0. & & & & & & & 1 & 21833 & 48644 \\
\hline 87 & 0 . & & & & & & & & 3071 & 01144 \\
\hline 89 & -0 & & & & & & & & 188 & 25513 \\
\hline 91 & -0 & & & & & & & & 6 & 87110 \\
\hline 93 & 0. & & & & & & & & & 24056 \\
\hline 95 & 0 . & & & & & & & & & 1244 \\
\hline 97 & -0 & & & & & & & & & 24 \\
\hline 99 & $\frac{-0}{0 .}$ & & & & & & & & & \\
\hline
\end{tabular}




\begin{tabular}{|c|c|c|c|c|c|c|c|c|c|c|}
\hline$k$ & & & & & $c_{k}^{(10)}$ & & & & & \\
\hline 0 & -0.00000 & 02000 & 10251 & 73332 & 51027 & 15374 & 94088 & 21393 & 15136 & 19071 \\
\hline 2 & 0. & 27478 & 75445 & 60047 & 12023 & 61586 & 71599 & 58275 & 49348 & 62984 \\
\hline 4 & -0 & 63539 & 01754 & 48107 & 93303 & 73621 & 62516 & 65088 & 09057 & 04745 \\
\hline 6 & 0 . & 61092 & 14465 & 70387 & 32368 & 86858 & 39303 & 76715 & 52180 & 67220 \\
\hline 8 & -0 & 10443 & 89166 & 71301 & 29573 & 54199 & 80515 & 30750 & 07693 & 40550 \\
\hline 10 & -0 & 47245 & 91519 & 14535 & 87378 & 92254 & 95603 & 48357 & 00079 & 14394 \\
\hline 12 & 0 . & 60374 & 95292 & 52658 & 06046 & 18847 & 42696 & 87220 & 03533 & 57458 \\
\hline 14 & -0 & 30087 & 99217 & 85345 & 07615 & 64329 & 85611 & 49262 & 29022 & 88521 \\
\hline 16 & -0 & 17 & 38736 & 80462 & 07477 & 34264 & 79937 & 76808 & 96123 & 34723 \\
\hline 18 & 0 . & 7045 & 81709 & 73958 & 10943 & 37790 & 22868 & 47494 & 05054 & 04702 \\
\hline 20 & -0 & 2446 & 38283 & 33826 & 78668 & 96084 & 49220 & 69614 & 22779 & 37509 \\
\hline 22 & -0 & 366 & 48190 & 58159 & 21954 & 34310 & 87613 & 50255 & 57531 & 71340 \\
\hline 24 & 0. & 333 & 51927 & 67091 & 53529 & 42073 & 16310 & 26163 & 73094 & 14643 \\
\hline 26 & -0 & 7 & 74280 & 84723 & 96860 & 11655 & 00926 & 31314 & 23690 & 91812 \\
\hline 28 & -0 & 22 & 37253 & 33674 & 46275 & 05608 & 65793 & 33717 & 05853 & 54060 \\
\hline 30 & 0 . & 1 & 34846 & 34487 & 79755 & 04149 & 83129 & 31907 & 21450 & 65827 \\
\hline 32 & 0 . & & 95245 & 81564 & 19560 & 50556 & 97858 & 67116 & 81001 & 47949 \\
\hline 34 & -0 & & 5135 & 48118 & 12690 & 14544 & 47420 & 42355 & 15107 & 23123 \\
\hline 36 & -0 & & 2772 & 57974 & 10706 & 80108 & 26809 & 33336 & 21170 & 80285 \\
\hline 38 & 0. & & 90 & 21412 & 02849 & 01953 & 90394 & 27088 & 34719 & 51164 \\
\hline 40 & 0. & & 56 & 64873 & 23277 & 31370 & 90095 & 04247 & 48049 & 27753 \\
\hline 42 & -0 & & & 54439 & 66659 & 04958 & 84764 & 11035 & 36560 & 76763 \\
\hline 44 & -0 & & & 82869 & 94363 & 83080 & 91093 & 53994 & 01205 & 83428 \\
\hline 46 & -0 & & & 877 & 70778 & 56500 & 68304 & 36711 & 29048 & 36232 \\
\hline 48 & 0. & & & 886 & 29188 & 84245 & 35191 & 18700 & 87906 & 09637 \\
\hline 50 & 0. & & & 24 & 87338 & 93748 & 77221 & 00292 & 35154 & 53111 \\
\hline 52 & -0 . & & & 7 & 06320 & 09941 & 91928 & 55176 & 95540 & 94142 \\
\hline 54 & -0 & & & & 31277 & 71304 & 03536 & 44064 & 06488 & 65497 \\
\hline 56 & 0. & & & & 4249 & 97771 & 81964 & 14544 & 07807 & 66204 \\
\hline 58 & 0 . & & & & 261 & 21183 & 03863 & 81995 & 13912 & 19411 \\
\hline 60 & -0 & & & & 19 & 34422 & 69225 & 28126 & 55000 & 29750 \\
\hline 62 & -0 & & & & 1 & 61109 & 84857 & 10036 & 81057 & 91010 \\
\hline 64 & 0. & & & & & 6513 & 56248 & 30117 & 46949 & 20131 \\
\hline 66 & 0 . & & & & & 771 & 65106 & 17734 & 30440 & 89520 \\
\hline 68 & -0 & & & & & 14 & 71798 & & & 85204 \\
\hline 70 & -0 & & & & & 2 & 95684 & 36851 & 81637 & 74345 \\
\hline 72 & 0. & & & & & & 1135 & 48296 & 02129 & 12023 \\
\hline 74 & 0. & & & & & & 924 & & 96848 & 34874 \\
\hline 76 & 0 . & & & & & & 7 & 60710 & 50040 & 70620 \\
\hline 78 & -0 & & & & & & 2 & 38561 & 26649 & 79935 \\
\hline 80 & -0 . & & & & & & & 4510 & 28551 & 99320 \\
\hline 82 & 0 . & & & & & & & 511 & 77082 & 87392 \\
\hline 84 & 0 . & & & & & & & 15 & 14546 & 38879 \\
\hline 86 & -0 & & & & & & & & 91127 & 25348 \\
\hline 88 & -0 . & & & & & & & & 3825 & 35878 \\
\hline 90 & 0. & & & & & & & & 132 & 81197 \\
\hline 92 & 0 . & & & & & & & & 7 & 85476 \\
\hline 94 & -0 & & & & & & & & & 15152 \\
\hline 96 & -0. & & & & & & & & & 1357 \\
\hline 98 & 0. & & & & & & & & & 12 \\
\hline 100 & 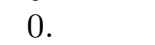 & & & & & & & & & \\
\hline
\end{tabular}


V. Tschebyscheffreihen der Koeffizienten $C_{n}(z)$

50 Dezimalstellen

$$
\begin{gathered}
(0 \leq n \leq 10) \\
C_{2 n}(z)=\sum_{k=0}^{\infty} \gamma_{2 k}^{(2 n)} T_{2 k}(z) \\
C_{2 n+1}(z)=z \sum_{k=0}^{\infty} \gamma_{2 k}^{(2 n+1)} T_{2 k}(z)
\end{gathered}
$$




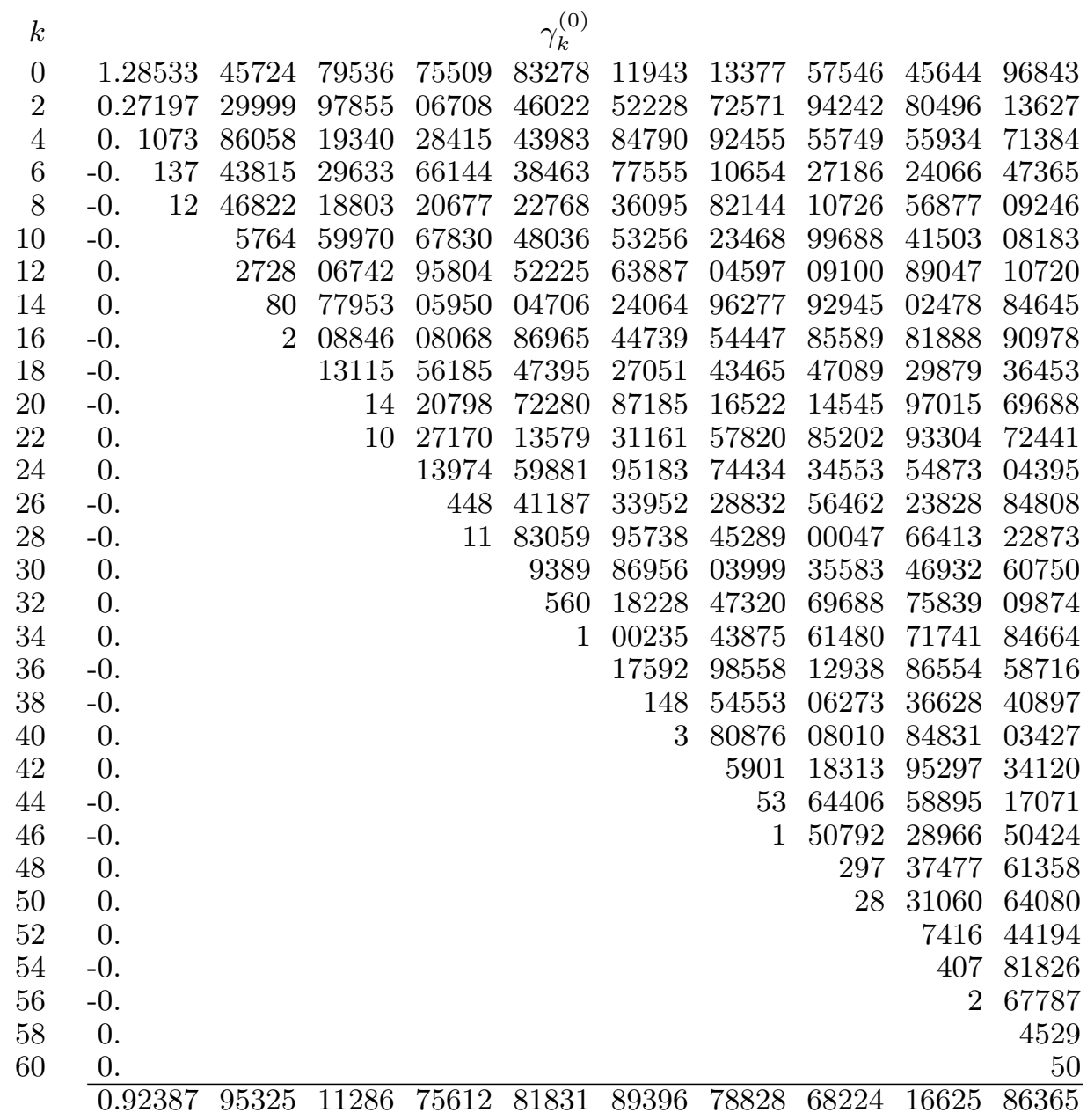




\begin{tabular}{|c|c|c|c|c|c|c|c|c|c|c|}
\hline$k$ & & & & & $\gamma_{k}^{(1)}$ & & & & & \\
\hline 0 & 0.00733 & 83131 & 24365 & 54395 & 58976 & 98324 & 10137 & 81951 & 44204 & 47791 \\
\hline 2 & -0. 2873 & 41409 & 66371 & 54549 & 72001 & 67056 & 57313 & 59859 & 04216 & 54515 \\
\hline 4 & $-0 . \quad 560$ & 71615 & 20384 & 22214 & 41208 & 97308 & 41266 & 81875 & 77875 & 51044 \\
\hline 6 & 0. & 07392 & 20807 & 27996 & 36562 & 21642 & 19569 & 24346 & 30365 & 48407 \\
\hline 8 & 0. & 20120 & 86631 & 27012 & 11396 & 51447 & 92331 & 65212 & 63719 & 94974 \\
\hline 10 & 0 . & 22058 & 23831 & 03165 & 28847 & 43848 & 82008 & 37534 & 77510 & 45286 \\
\hline 12 & -0 & 1090 & 73857 & 68109 & 82094 & 76299 & 59085 & 54995 & 17626 & 67371 \\
\hline 14 & -0 & 86 & 55485 & 64945 & 32274 & 27646 & 08265 & 21725 & 88612 & 99420 \\
\hline 16 & 0. & & 9551 & 11244 & 76693 & 21632 & 11988 & 42623 & 31828 & 30816 \\
\hline 18 & 0. & & 13188 & 07072 & 88781 & 01857 & 97837 & 74092 & 13017 & 93056 \\
\hline 20 & 0. & & 211 & 59709 & 18325 & 53103 & 90394 & 30269 & 08750 & 27148 \\
\hline 22 & -0 & & 9 & 99713 & 87763 & 83605 & 71606 & 02408 & 24818 & 75785 \\
\hline 24 & -0. & & & 30783 & 72420 & 60626 & 88279 & 03743 & 53814 & 01242 \\
\hline 26 & 0 . & & & 349 & 81526 & 23887 & 46286 & 29201 & 28823 & 05414 \\
\hline 28 & 0. & & & 22 & 57403 & 42849 & 95734 & 63953 & 90895 & 33393 \\
\hline 30 & 0. & & & & 2965 & 80834 & 96795 & 90146 & 33431 & 11313 \\
\hline 32 & -0 & & & & 1035 & 74705 & 43081 & 69452 & 23661 & 56997 \\
\hline 34 & -0 . & & & & 9 & 57508 & 27773 & 53987 & 62107 & 60197 \\
\hline 36 & 0. & & & & & 31498 & 46681 & 31185 & 26787 & 66521 \\
\hline 38 & 0. & & & & & 537 & 74438 & 45416 & 82810 & 03031 \\
\hline 40 & -0 . & & & & & 6 & 10338 & 89042 & 62897 & 80770 \\
\hline 42 & -0 . & & & & & & 18459 & 66984 & 45692 & 43165 \\
\hline 44 & 0. & & & & & & 50 & 17972 & 75987 & 45493 \\
\hline 46 & 0 . & & & & & & 4 & 51972 & 42434 & 43113 \\
\hline 48 & 0. & & & & & & & 1171 & 69478 & 88276 \\
\hline 50 & -0 . & & & & & & & 83 & 08858 & 95157 \\
\hline 52 & -0. & & & & & & & & 57972 & 01462 \\
\hline 54 & 0. & & & & & & & & 1158 & 70477 \\
\hline 56 & 0. & & & & & & & & 13 & 71707 \\
\hline$\pi$ & -0 . & & & & & & & & & 11819 \\
\hline 60 & -0. & & & & & & & & & 231 \\
\hline 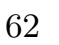 & 0 . & & & & & & & & & 1 \\
\hline
\end{tabular}




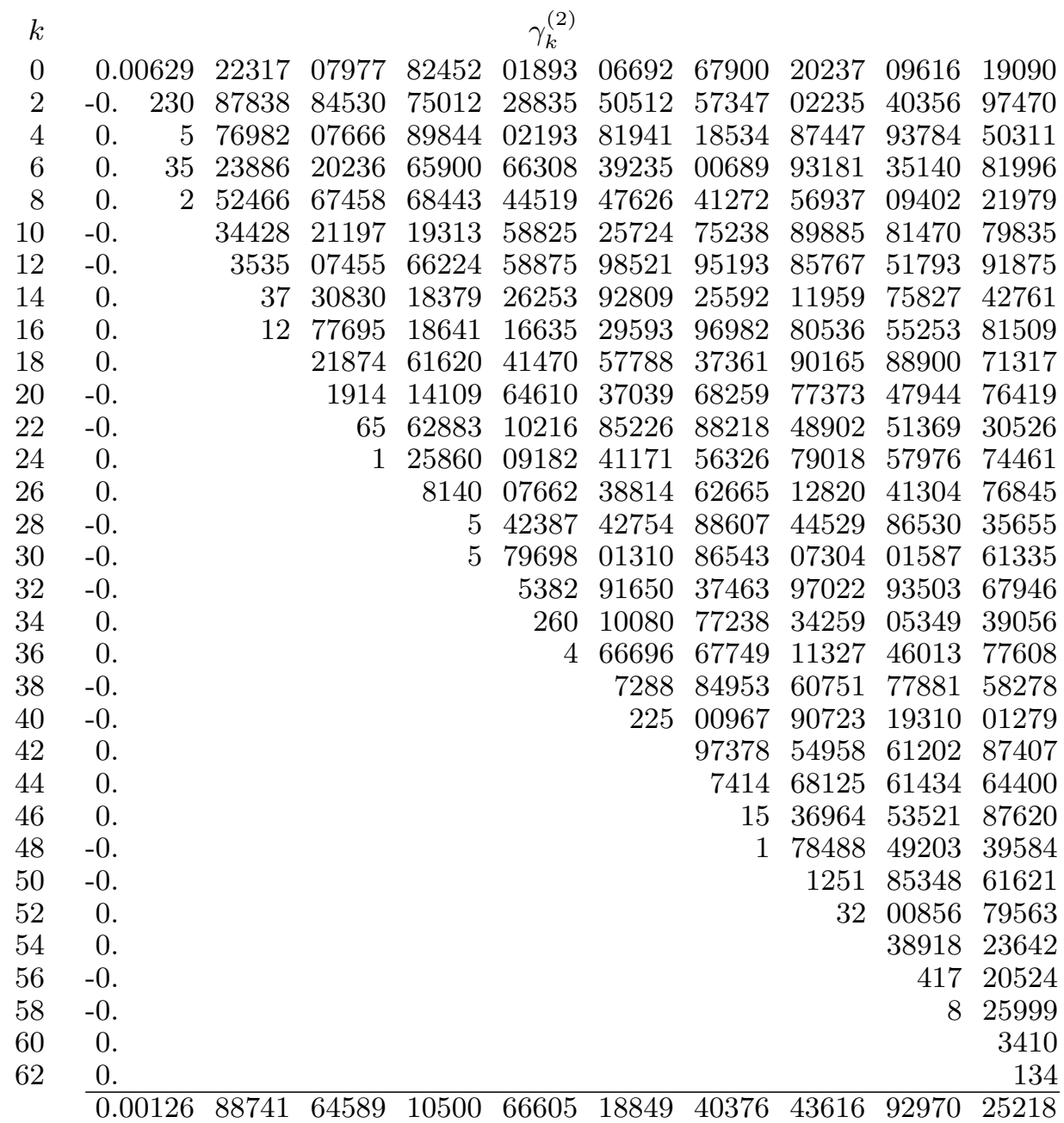




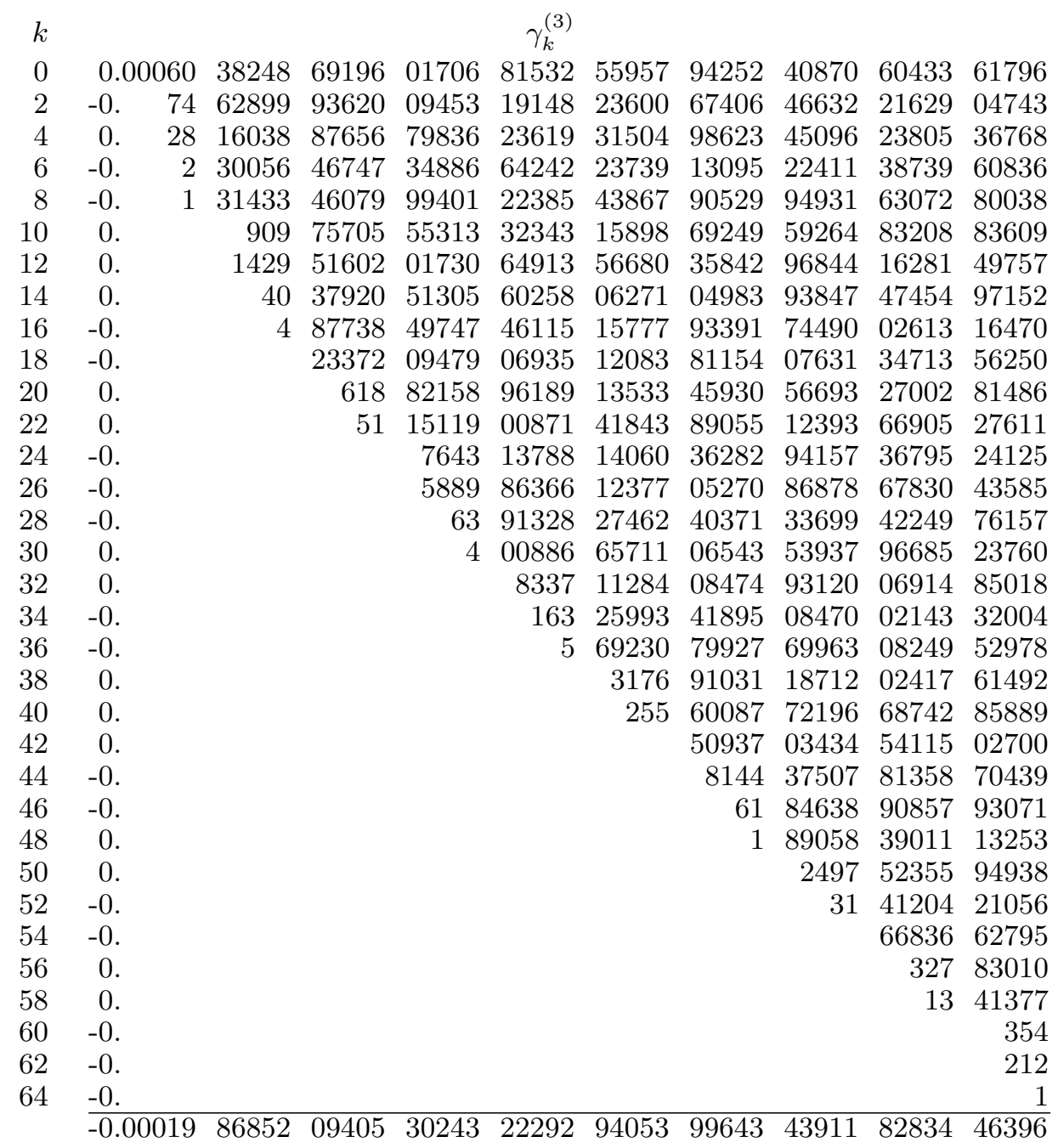




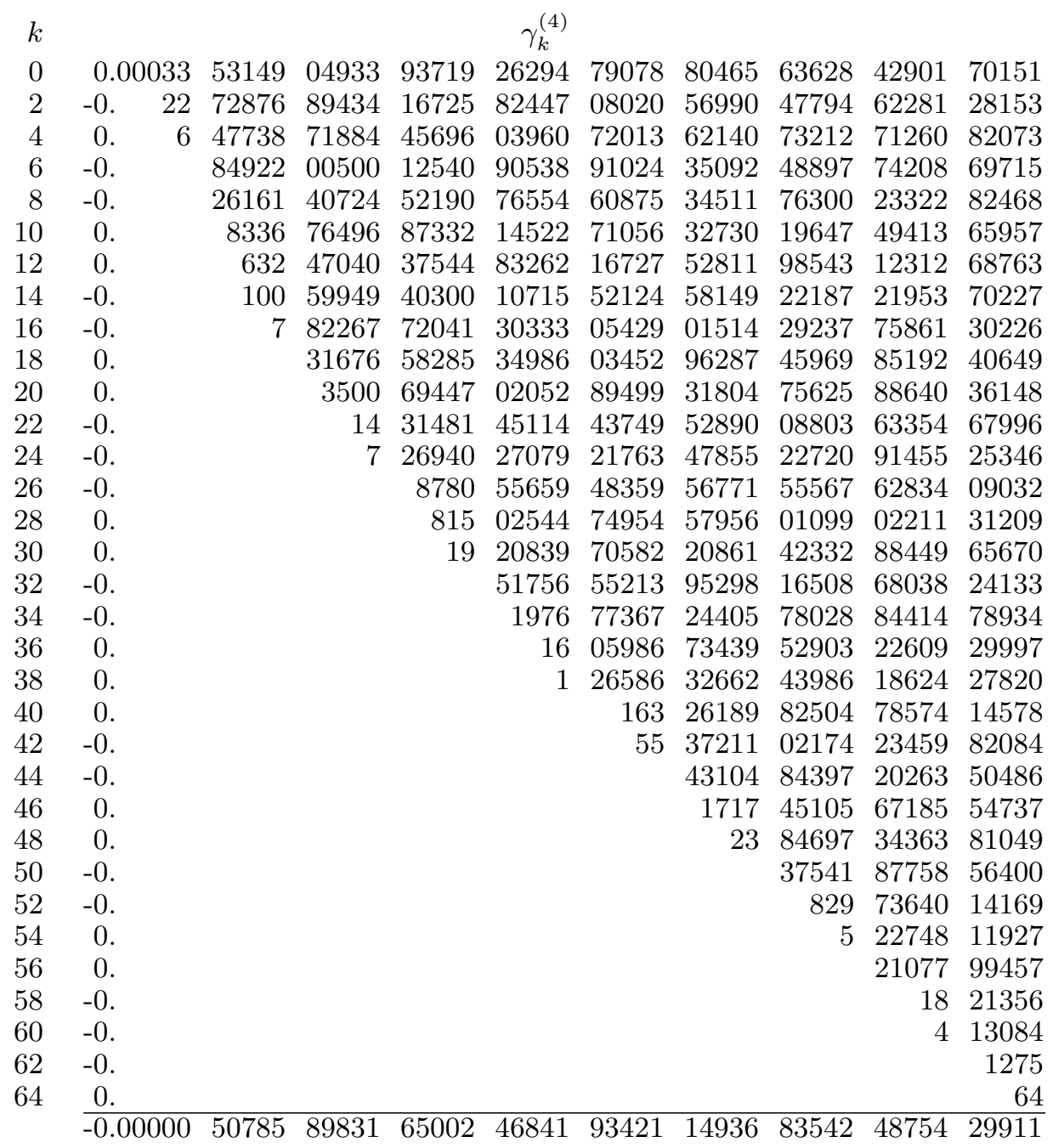




\begin{tabular}{|c|c|c|c|c|c|c|c|c|c|c|}
\hline$k$ & & & & & $\gamma_{k}^{(5)}$ & & & & & \\
\hline 0 & -0.00020 & 18981 & 43328 & 10260 & 77235 & 33269 & 94906 & 13047 & 23554 & 33479 \\
\hline 2 & 0. & 53212 & 38631 & 92457 & 79320 & 01755 & 45660 & 47611 & 63167 & 10837 \\
\hline 4 & 0. & 59361 & 31306 & 73219 & 68818 & 16044 & 81728 & 10544 & 71778 & 98947 \\
\hline 6 & -0 . & 55692 & 82352 & 78899 & 52063 & 19394 & 52518 & 43828 & 10579 & 58463 \\
\hline 8 & 0. & 13498 & 28777 & 80148 & 68715 & 92564 & 27526 & 81328 & 91545 & 24235 \\
\hline 10 & -0 & 184 & 25542 & 98220 & 93803 & 61225 & 09022 & 51953 & 94136 & 40546 \\
\hline 12 & -0 & 370 & 03939 & 42792 & 74794 & 78174 & 78072 & 85862 & 77641 & 44904 \\
\hline 14 & 0. & 7 & 81440 & 67639 & 77286 & 50908 & 44363 & 71189 & 22516 & 12383 \\
\hline 16 & 0. & 3 & 71737 & 48594 & 54668 & 13489 & 74903 & 26739 & 00182 & 31764 \\
\hline 18 & 0. & & 1763 & 18257 & 61962 & 34236 & 46726 & 81996 & 05981 & 04409 \\
\hline 20 & -0 . & & 1542 & 15039 & 78543 & 73806 & 54182 & 32898 & 10768 & 25111 \\
\hline 22 & -0 & & 31 & 97827 & 57569 & 90931 & 06161 & 60716 & 29942 & 86178 \\
\hline 24 & 0. & & 3 & 06157 & 37656 & 80689 & 65881 & 11830 & 55320 & 48558 \\
\hline 26 & 0. & & & 10134 & 46160 & 41216 & 39524 & 28822 & 73595 & 64347 \\
\hline 28 & -0 & & & 313 & 18394 & 33940 & 60341 & 91384 & 08290 & 33667 \\
\hline 30 & -0 & & & 15 & 70008 & 10192 & 75403 & 98283 & 11813 & 06290 \\
\hline 32 & 0 . & & & & 14404 & 51842 & 28939 & 64225 & 34896 & 74653 \\
\hline 34 & 0. & & & & 1459 & 93533 & 64699 & 04948 & 78514 & 68051 \\
\hline 36 & 0. & & & & 2 & 59608 & 49210 & 55653 & 72586 & 39436 \\
\hline 38 & -0 . & & & & & 89294 & 42752 & 80508 & 90540 & 80832 \\
\hline 40 & -0 . & & & & & 819 & 17045 & 73158 & 24529 & 73938 \\
\hline 42 & 0 . & & & & & 37 & 49628 & 41308 & 81267 & 94351 \\
\hline 44 & 0. & & & & & & 59859 & 60155 & 14778 & 50000 \\
\hline 46 & -0 & & & & & & 1081 & 08673 & 82178 & 20610 \\
\hline 48 & -0 & & & & & & 27 & 00212 & 66774 & 34829 \\
\hline 50 & 0. & & & & & & & 19502 & 83166 & 97012 \\
\hline 52 & 0. & & & & & & & 872 & 02698 & 26323 \\
\hline 54 & -0 & & & & & & & & 93522 & 70445 \\
\hline 56 & -0 & & & & & & & & 21352 & 77170 \\
\hline 58 & -0 & & & & & & & & 70 & 77979 \\
\hline 60 & 0. & & & & & & & & 4 & 07047 \\
\hline 62 & 0. & & & & & & & & & 2912 \\
\hline 64 & -0 & & & & & & & & & 61 \\
\hline . & $\underline{-0 .}$ & & & & & & & & & \\
\hline
\end{tabular}




\begin{tabular}{|c|c|c|c|c|c|c|c|c|c|c|}
\hline$k$ & & & & & $\gamma_{k}^{(6)}$ & & & & & \\
\hline 0 & 0.00002 & 43794 & 84282 & 13794 & 12901 & 48074 & 79635 & 35212 & 83927 & 13064 \\
\hline 2 & -0 & 38297 & 60140 & 50378 & 67038 & 36744 & 01916 & 31749 & 77066 & 61864 \\
\hline 4 & 0. & 51109 & 67304 & 99826 & 00183 & 39455 & 97485 & 28054 & 12596 & 81576 \\
\hline 6 & -0 & 20458 & 13645 & 03860 & 76147 & 44038 & 37477 & 57424 & 40824 & 98148 \\
\hline 8 & 0. & 4938 & 13664 & 48320 & 11752 & 89782 & 84032 & 07758 & 19285 & 76727 \\
\hline 10 & -0 & 361 & 87528 & 34962 & 28140 & 71368 & 61138 & 16473 & 51537 & 90405 \\
\hline 12 & -0 & 128 & 76905 & 09807 & 98607 & 19900 & 46819 & 27486 & 63650 & 18126 \\
\hline 14 & 0. & 25 & 74412 & 11114 & 48661 & 81986 & 59212 & 23907 & 50240 & 52255 \\
\hline 16 & 0. & 1 & 36414 & 57070 & 79168 & 35873 & 60473 & 64886 & 55148 & 62795 \\
\hline 18 & -0 & & 30324 & 39574 & 08438 & 20831 & 12986 & 00877 & 50716 & 82154 \\
\hline 20 & -0 & & 1321 & 66712 & 39902 & 53693 & 45498 & 24918 & 43540 & 54071 \\
\hline 22 & 0. & & 130 & 31652 & 13000 & 93681 & 30207 & 50614 & 56813 & 99699 \\
\hline 24 & 0. & & 6 & 63588 & 35532 & 00669 & 90941 & 11267 & 59194 & 05186 \\
\hline 26 & -0 & & & 24600 & 35654 & 79328 & 00436 & 71027 & 52605 & 50906 \\
\hline 28 & -0 & & & 1681 & 52792 & 08168 & 83360 & 20027 & 27606 & 95137 \\
\hline 30 & 0. & & & 18 & 93793 & 20803 & 59403 & 33971 & 43924 & 24567 \\
\hline 32 & 0. & & & 2 & 43065 & 06127 & 37235 & 99200 & 17745 & 19625 \\
\hline 34 & 0. & & & & 460 & 84861 & 41193 & 19911 & 36630 & 36440 \\
\hline 36 & -0 & & & & 219 & 56897 & 62633 & 71151 & 35571 & 13921 \\
\hline 38 & -0 & & & & 2 & 29588 & 03325 & 96836 & 94725 & 47682 \\
\hline 40 & 0. & & & & & 13065 & 99063 & 38240 & 80331 & 95096 \\
\hline 42 & 0. & & & & & 234 & 71644 & 89511 & 60725 & 79122 \\
\hline 44 & -0 & & & & & 5 & 18149 & 96672 & 99090 & 70426 \\
\hline 46 & -0 & & & & & & 14258 & 92256 & 16492 & 47277 \\
\hline 48 & 0 & & & & & & 127 & 33209 & 77817 & 00606 \\
\hline 50 & 0. & & & & & & 6 & 02914 & 39303 & 16113 \\
\hline 52 & -0 & & & & & & & 1032 & 75494 & 24628 \\
\hline 54 & -0 & & & & & & & 189 & 01407 & 75501 \\
\hline 56 & -0 & & & & & & & & 61705 & 08484 \\
\hline 58 & 0. & & & & & & & & 4530 & 98979 \\
\hline 60 & 0 & & & & & & & & 33 & 86503 \\
\hline 62 & -0. & & & & & & & & & 83932 \\
\hline 64 & -0 & & & & & & & & & 1001 \\
\hline 66 & 0. & & & & & & & & & 12 \\
\hline & & & & & & & & & & \\
\hline
\end{tabular}




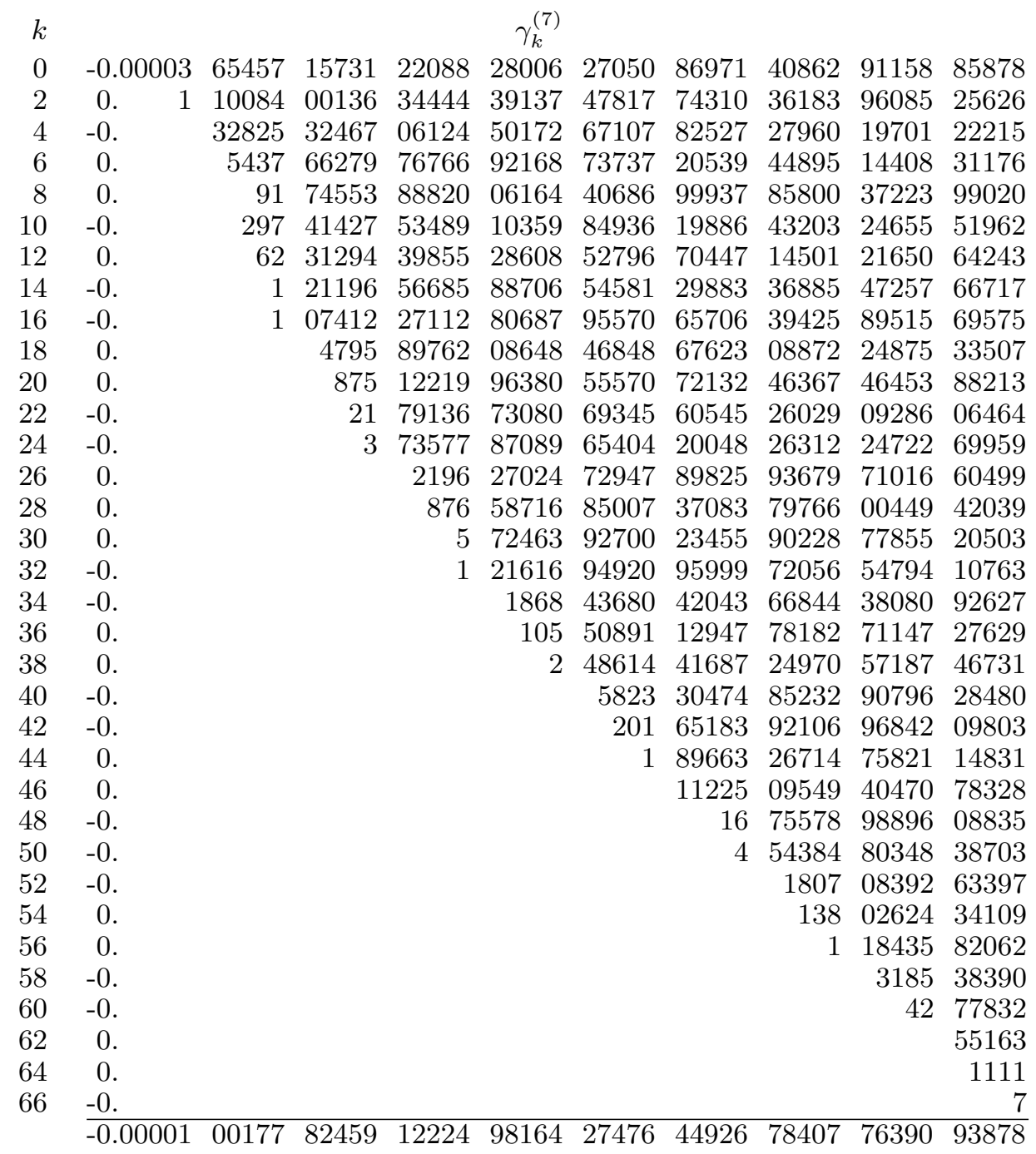




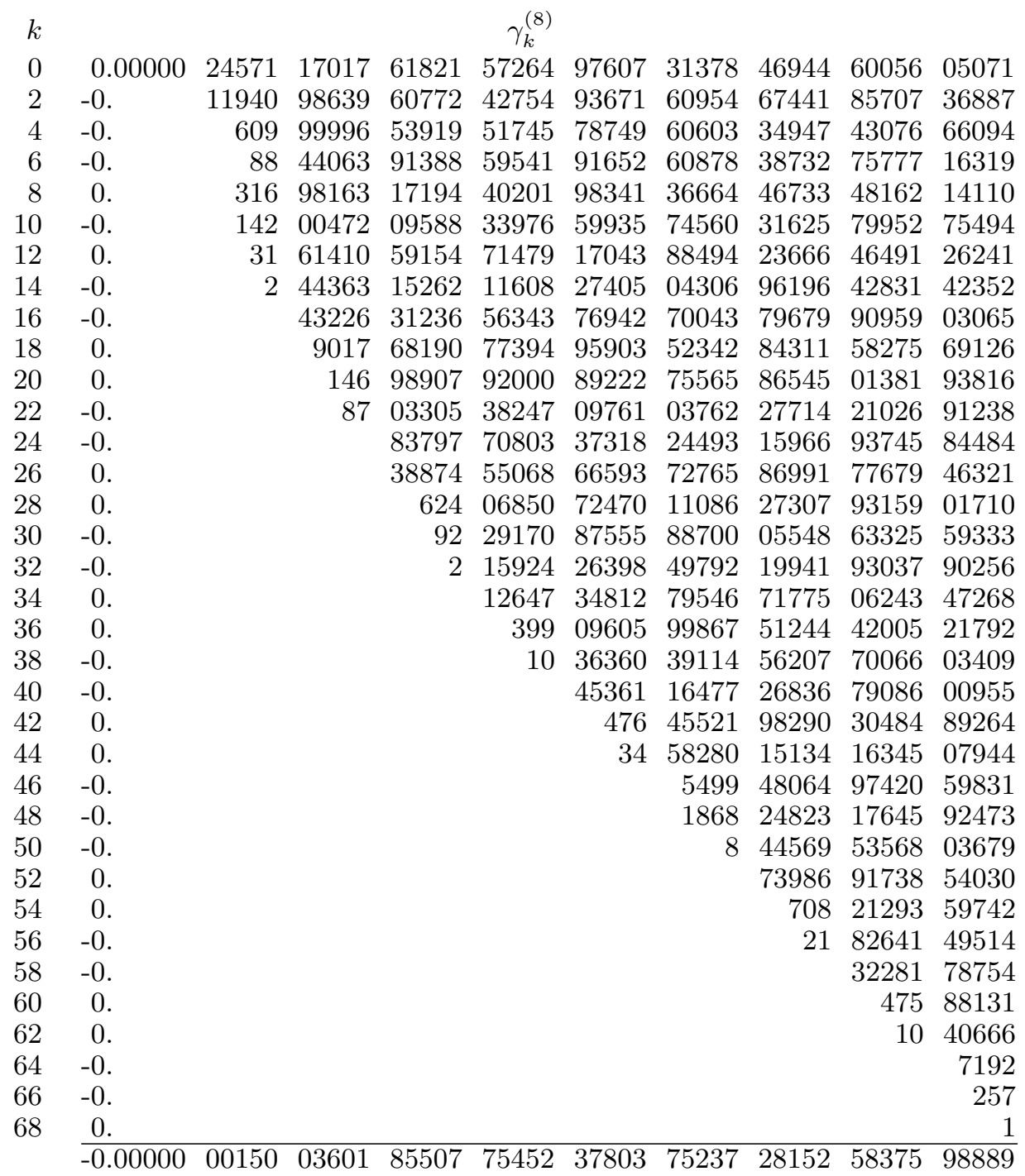




\begin{tabular}{|c|c|c|c|c|c|c|c|c|c|c|}
\hline$k$ & & & & & $\gamma_{k}^{(9)}$ & & & & & \\
\hline 0 & -0.00000 & 80668 & 22285 & 19551 & 64615 & 97584 & 32896 & 82516 & 72477 & 15889 \\
\hline 2 & 0. & 20252 & 28197 & 48693 & 05969 & 04605 & 96692 & 37927 & 92096 & 06667 \\
\hline 4 & -0 & 6113 & 23732 & 62780 & 19174 & 19181 & 47502 & 83972 & 90048 & 83653 \\
\hline 6 & 0. & 1689 & 93326 & 57723 & 01425 & 86354 & 20981 & 07018 & 41181 & 45772 \\
\hline 8 & -0 & 386 & 77374 & 32115 & 02548 & 99754 & 67222 & 89638 & 62368 & 08400 \\
\hline 10 & 0 . & 62 & 59906 & 35892 & 67559 & 85981 & 02442 & 86472 & 26093 & 78189 \\
\hline 12 & -0 & 3 & 62846 & 69051 & 52954 & 64971 & 87207 & 44261 & 60910 & 96341 \\
\hline 14 & -0 & 1 & 08059 & 05023 & 26490 & 52614 & 15408 & 12705 & 37400 & 38335 \\
\hline 16 & 0. & & 27038 & 40332 & 23753 & 07283 & 23036 & 25334 & 13647 & 72756 \\
\hline 18 & -0 & & 1225 & 12678 & 73263 & 31021 & 57506 & 34740 & 14889 & 12376 \\
\hline 20 & -0 & & 278 & 53879 & 78776 & 89154 & 47490 & 36769 & 08912 & 12487 \\
\hline 22 & 0. & & 22 & 15543 & 70252 & 17402 & 01074 & 99118 & 69479 & 62959 \\
\hline 24 & 0. & & 1 & 63940 & 47879 & 14063 & 94917 & 43450 & 69621 & 99822 \\
\hline 26 & -0 & & & 11419 & 33819 & 81940 & 77210 & 01705 & 12926 & 88943 \\
\hline 28 & -0 & & & 647 & 72080 & 44847 & 33742 & 22000 & 62828 & 70284 \\
\hline 30 & 0. & & & 27 & 68618 & 40083 & 59666 & 39960 & 59644 & 74530 \\
\hline 32 & 0 . & & & 1 & 62743 & 67431 & 60784 & 26797 & 48066 & 76288 \\
\hline 34 & -0 . & & & & 3516 & 44658 & 52929 & 68997 & 07480 & 46945 \\
\hline 36 & -0 & & & & 260 & 24300 & 24497 & 25837 & 38639 & 18510 \\
\hline 38 & 0. & & & & 2 & 12805 & 41005 & 63576 & 10921 & 71125 \\
\hline 40 & 0. & & & & & 27577 & 28585 & 46395 & 37131 & 18391 \\
\hline 42 & 0. & & & & & 14 & 96365 & 96357 & 59272 & 06186 \\
\hline 44 & -0 . & & & & & 20 & 21137 & 45024 & 93025 & 54844 \\
\hline 46 & -0 & & & & & & 13858 & 41819 & 24883 & 82267 \\
\hline 48 & 0. & & & & & & 1058 & 28143 & 65469 & 17763 \\
\hline 50 & 0. & & & & & & 12 & 97167 & 89429 & 01193 \\
\hline 52 & -0 & & & & & & & 40273 & 75071 & 82999 \\
\hline 54 & -0 & & & & & & & 725 & 68559 & 65025 \\
\hline 56 & 0. & & & & & & & 11 & 05724 & 98315 \\
\hline 58 & 0. & & & & & & & & 28888 & 59700 \\
\hline 60 & -0 & & & & & & & & 204 & 32158 \\
\hline 62 & -0 & & & & & & & & 8 & 74574 \\
\hline 04 & 0. & & & & & & & & & 1666 \\
\hline$c$ & 0. & & & & & & & & & 8 \\
\hline & & & & & & & & & & \\
\hline
\end{tabular}




\begin{tabular}{|c|c|c|c|c|c|c|c|c|c|c|}
\hline$k$ & & & & & $\gamma_{k}^{(10)}$ & & & & & \\
\hline 0 & 0.00000 & 01396 & 23158 & 56448 & 96271 & 67546 & 87884 & 77148 & 69664 & 29684 \\
\hline 2 & 0 & 518 & 76020 & 99781 & 90896 & 29148 & 36083 & 77933 & 80205 & 15311 \\
\hline 4 & -0 & 1502 & 56894 & 00416 & 70346 & 78130 & 02704 & 42265 & 73327 & 93564 \\
\hline 6 & 0 & 538 & 51754 & 15429 & 12940 & 18591 & 96330 & 90906 & 69147 & 98767 \\
\hline 8 & -0 & 120 & 09470 & 94721 & 26665 & 15285 & 93225 & 00831 & 32425 & 86715 \\
\hline 10 & 0 & 18 & 44141 & 61121 & 34064 & 96087 & 76058 & 65392 & 48900 & 90575 \\
\hline 12 & -0 & & 60512 & 85922 & 58187 & 97076 & 61529 & 03233 & 67566 & 76416 \\
\hline 14 & -0 & & 58913 & 92764 & 47941 & 41266 & 33294 & 56283 & 35090 & 89308 \\
\hline 16 & 0 & & 16515 & 77264 & 14351 & 16930 & 87034 & 29790 & 16875 & 61022 \\
\hline 18 & -0 & & 1648 & 99182 & 75452 & 74252 & 00545 & 98466 & 11038 & 37229 \\
\hline 20 & -0 & & 84 & 50007 & 40924 & 13967 & 84374 & 10529 & 40614 & 33412 \\
\hline 22 & 0 & & 30 & 23518 & 01777 & 26554 & 44814 & 93791 & 80963 & 90116 \\
\hline 24 & -0 & & & 61792 & 01123 & 77458 & 04263 & 61173 & 49740 & 30616 \\
\hline 26 & -0 & & & 21506 & 48020 & 78085 & 27206 & 14695 & 97726 & 02171 \\
\hline 28 & 0 & & & 523 & 60584 & 16945 & 07406 & 26560 & 62805 & 15936 \\
\hline 30 & 0 & & & 87 & 02944 & 99075 & 88985 & 84072 & 59778 & 59515 \\
\hline 32 & -0 & & & 1 & 27211 & 27494 & 56193 & 42301 & 81121 & 40766 \\
\hline 34 & -0 & & & & 21508 & 80677 & 13936 & 63172 & 03462 & 92080 \\
\hline 36 & 0. & & & & 94 & 42732 & 61756 & 40684 & 31763 & 38771 \\
\hline 38 & 0. & & & & 33 & 86918 & 51579 & 23337 & 51053 & 74935 \\
\hline 40 & 0. & & & & & 12242 & 35865 & 98890 & 73712 & 39085 \\
\hline 42 & -0 & & & & & 3547 & 58606 & 57436 & 26225 & 66963 \\
\hline 44 & -0 & & & & & 35 & 15406 & 11385 & 45924 & 75071 \\
\hline 46 & 0. & & & & & 2 & 56244 & 50392 & 46635 & 39942 \\
\hline 48 & 0. & & & & & & 3982 & 56508 & 00273 & 14066 \\
\hline 50 & -0 & & & & & & 130 & 43413 & 38490 & 64986 \\
\hline 52 & -0 . & & & & & & 2 & 83801 & 99311 & 09570 \\
\hline 54 & 0. & & & & & & & 4662 & 70139 & 47129 \\
\hline 56 & 0. & & & & & & & 143 & 45489 & 66385 \\
\hline 58 & -0. & & & & & & & 1 & 09390 & 81899 \\
\hline 60 & -0 . & & & & & & & & 5446 & 16726 \\
\hline 62 & 0. & & & & & & & & 10 & 84882 \\
\hline 64 & 0. & & & & & & & & 1 & 60596 \\
\hline 66 & 0. & & & & & & & & & 344 \\
\hline 68 & -0 & & & & & & & & & 38 \\
\hline
\end{tabular}




\section{Lebenslauf}

Am 22.02.1949 wurde ich, Wolfgang Gabcke, als Sohn des Lehrers Harry Gabcke und seiner Ehefrau, der Lehrerin Hildegard Gabcke, geb. Bensel, in Bremerhaven geboren. Ich besitze die deutsche Staatsangehörigkeit.

Ab Ostern 1955 besuchte ich die Altwulsdorfer Schule und ab Ostern 1959 den altsprachlichen Zweig des Gymnasiums der WilhelmRaabe-Schule in Bremerhaven. Am 22.06.1967 legte ich dort die Reifeprüfung ab.

Nach zweijähriger freiwilliger Dienstzeit beim Bundesgrenzschutz immatrikulierte ich mich am 01.10.1969 an der Georg-August-Universität zu Göttingen im Fach Mathematik mit Nebenfach Physik. Nach der Vordiplomprüfung am 27.10.1971 bestand ich am 24.10.1975 die Hauptdiplomprüfung in dieser Fachkombination.

Seit dem 02.10.1978 bin ich im Angestelltenverhältnis bei einem in Göttingen ansässigen Versicherungsunternehmen beschäftigt. 